\title{
Unmet reproductive health care needs among rural Ghanaian women
}

Citation for published version (APA):

Ayanore, M. A. (2017). Unmet reproductive health care needs among rural Ghanaian women. [Doctoral Thesis, Maastricht University]. Maastricht University. https://doi.org/10.26481/dis.20170707ma

Document status and date:

Published: 01/01/2017

DOI:

10.26481/dis.20170707ma

Document Version:

Publisher's PDF, also known as Version of record

\section{Please check the document version of this publication:}

- A submitted manuscript is the version of the article upon submission and before peer-review. There can be important differences between the submitted version and the official published version of record.

People interested in the research are advised to contact the author for the final version of the publication, or visit the DOI to the publisher's website.

- The final author version and the galley proof are versions of the publication after peer review.

- The final published version features the final layout of the paper including the volume, issue and page numbers.

Link to publication

\footnotetext{
General rights rights.

- You may freely distribute the URL identifying the publication in the public portal. please follow below link for the End User Agreement:

www.umlib.nl/taverne-license

Take down policy

If you believe that this document breaches copyright please contact us at:

repository@maastrichtuniversity.nl

providing details and we will investigate your claim.
}

Copyright and moral rights for the publications made accessible in the public portal are retained by the authors and/or other copyright owners and it is a condition of accessing publications that users recognise and abide by the legal requirements associated with these

- Users may download and print one copy of any publication from the public portal for the purpose of private study or research.

- You may not further distribute the material or use it for any profit-making activity or commercial gain

If the publication is distributed under the terms of Article $25 \mathrm{fa}$ of the Dutch Copyright Act, indicated by the "Taverne" license above, 
ISBN: 9789461597229

Unmet reproductive health care needs among rural Ghanaian women

(C) Martin Amogre Ayanore, 2017

Cover page and layout designed by BRANDHAUZ ADS Ghana $+2330244053131$

Printed by Datawyse

All rights reserved. No part of this publication may be reproduced, stored in a retrieval system, or transmitted in any form or by any means, electronic, mechanical, photocopying, recording or otherwise, without the written permission from the author. 


\title{
Unmet reproductive health care needs among rural Ghanaian women
}

\author{
Dissertation
}

to obtain the degree of Doctor at Maastricht University, on the authority of the Rector Magnificus, Prof. dr. Rianne M. Letschert in accordance with the decision of the board of the Deans, to be defended in public on Friday 7 July, 2017, at 10:00 hours

by

Martin Amogre Ayanore 


\section{Promoter:}

Prof. dr. Wim Groot

Co-promoter:

Dr. Milena Pavlova

\section{Assessment Committee:}

Professor Hans Bosma (Chair), Maastricht University

Professor Philip Baba Adongo, University of Ghana

Professor Sjaak van der Geest, University of Amsterdam

Professor Rob Ruiter, Maastricht University

Professor Hein de Vries, Maastricht University

\section{Acknowledgement of funding}

The research presented in this dissertation is made possible through sponsorship from the Ministry of Energy capacity building scholarship scheme, Ghana for Martin Amogre Ayanore PhD studies with University of Maastricht, Netherlands. Additional budgetary support from the Centre for Health Policy Advocacy, Innovation \& Research in Africa (CHPAIR-Africa), Accra, Ghana provided additinal research funding to make this work possible. All views presented in this dissertation represent solely that of the author, and do not reflect in any way views from any of the above institutions.

\section{Maastricht University}

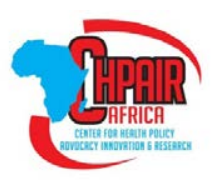




\section{Contents of Dissertation}

Chapter 1 General Introduction 9

Chapter 2 Unmet Reproductive Health Needs among Women in Some West African Countries: A Systematic Review of Outcome Measures and Determinants

Chapter 3 Focused maternity care in Ghana: results of a cluster analysis

Chapter 4 Stakeholders' views on maternity care shortcomings in rural Ghana: An ethnographic study among women, providers, policy makers, and quasi-private sector actors

Chapter 5 Context-specific factors and contraceptive use: accounts of women, men and health providers in a rural Ghanaian district

Chapter 6 Stakeholder's experiences, expectations and decision making on reproductive care: An ethnographic study of three districts in northern Ghana

Chapter 7 General Discussion

References

Appendix A

Appendix B

Appendix C

Appendix D

199

Summary

Acknowledgement

Curriculum Vitae

Publications 


\section{List of Tables}

2.1 Cross tabulations of outcome measures of and reproductive health need at three levels in reviewed publications

2.2 Extent of unmet needs per outcome measures in countries in review

2.3 Cross tabulations of determinants and reproductive health need at three levels in reviewed publications

3.1 Households distribution and enumeration areas in the 2008 GDHS

3.2 Regression results of cluster membership groups with individual, household and national explanatory variables 58

4.1 Participant and study setting characteristics

4.2 Inductive thematic processes and final themes that emerged among all stakeholder groups

5.1 Brief profile of qualitative demographics in study

5.2 List of main questions included in the FGDs and IDI guide

5.3 Background characteristics of survey respondents interviewed

5.4 Multinomial logistic regression of current and ever use, access on demand and future contraceptive use with individual and household explanatory variables

6.1 Women views on gender role impacts on attaining their reproductive care needs

6.2 Stakeholder experiences with service provision to meet user reproductive preferences

6.3 Stakeholder expectations on provision and meeting women reproductive care preferences

6.4 Policy setting and decision making expressions in meeting women reproductive preferences 


\section{List of Figures}

1.1 Unweighted means for global trends of unmet need for family planning.

1.2 Met and unmet care demands across selected West African Countries from DHS surveys (2011-2014)

1.3 Percentage of married or in union women with unmet need for family planning in selected West African countries from 1990-2014.

1.4 Total health expenditures (\% of GDP) in selected developing countries

$1.5 \quad$ Health expenditure per capita (current US\$) in selected West African Countries

1.6 Out-of-pocket health expenditure (\% of private expenditure on health) trends across selected African countries

1.7 Maternity care trends across selected indicators in Ghana from 1988-2014

2.1 Main key words (left) and flow diagram (right) of systematic review process 32

3.1 Overall distribution of respondents in the cluster analysis 53

3.2 Overall levels of care components received by women (15-49 years)

3.3 Prenatal and postnatal care cluster characteristics for women (15-49 years)

3.4 Facility utilization and other care components received by women (15-49 years)

3.5 Medication care components received by women respondents (15-49 years) 


\section{List of Boxes}

2.1 Main key words applied in the literature search for all databases 32

3.1 Components of WHO "focused care" and Ghana success maternity interventions

4.1 Final emergent theme categories among all stakeholder groups from the study 78

5.1 Expressions of current and ever uses for contraception 96

5.2 Key thematic expressions on contraceptive access on demand and future use intentions 98

6.1 Sample question used to assess all stakeholder groups 110 


\section{CHAPTER 1}

General Introduction 


\subsection{Scope of the dissertation}

After Ghana's independence in 1957, healthcare became state funded. Government invested in health care infrastructure and skilled professional care to meet growing healthcare demands (Baidoo, 2009; Frimpong, 2013; Opare \& Mill, 2000; Senah, 2001). Government's priority focus was to streamline health service delivery and improve access for every citizen (Frimpong, 2013; Opare \& Mill, 2000; Senah, 2001; Waddington \& Enyimayew, 1990). Government health care expenditure thus increased from 6.4\% of GDP in 1965 to $8.2 \%$ of GDP in 1969 (Frimpong, 2013; Patterson, 1981), with a phenomenal increase in the number of health facilities (Frimpong, 2013; Twumasi, 1981). However, political and economic instability in the country worsened the quality and delivery of health care in the late 1960s (Twumasi, 1981). The continual decline of economic conditions in the 1980s, forced the introduction of the hospital fee legislation in 1985. Subsequently, a full fee cost recovery program "cash and carry" became operational in 1992. This legislation provided partial and full coverage of payments for consultation/diagnosis and drugs (Asenso-Okyere, Anum, Osei-Akoto, \& Adukonu, 1998). However, this did not solve the problem of rising household expenditures to meet individual care demands post-independence.

The catastrophic expenditures and rising unmet health gaps were recognized in the first population policy in 1969. Following the 1987 Safe Motherhood conference in Nairobi and the International Conference on Population and Development (ICPD, 1994) in Cairo, Ghana initiated targeted interventions to improve maternity and reproductive health services provision. The country's first national health insurance policy; the National Health Insurance Scheme (NHIS) was passed into law in the year 2003. The rational was to provide a new pathway for funding healthcare, improving healthcare access while shouldering the depleting effects caused by "cash and carry system". Results have shown consistent improvement in health care access over the last decade after the schemes introduction. Key maternity indicators assessed the Demographic Health Surveys (DHS) for 2008 and 2014 following the introduction of NHIS show improvements for key maternity care indicators. In particular, growing met needs for facilitybased birthing, postnatal care, and reduced maternal mortality rates are known. Additionally, increasing ownership and use of social health insurance for accessing healthcare services are also evidenced (see Figure 1.8). DHS findings in Ghana also show consistent increases in satisfied demands for modern contraceptive use between 1998-2014 (GSS, GHS, \& ICF, 2014). Notwithstanding, barriers for women's access and utilization of reproductive and maternal health services are increasingly reported (Atinga \& Baku, 2013; Josephine Borghi et al., 2003; Filippi et al., 2006; Ganle, Parker, Fitpatrick, \& Otupiri, 2015; Perkins et al., 2009; Witter, 
D. K., Arhinful, A. Kusi, \& Zakariah-Akoto, 2007). Inadequate obstetric care and poor health staff-patient relations and its impacts on maternity service outcomes is evident in Ghana (Ganle et al., 2015). Poor contraceptive access and inconsistent use of contraceptives are known among adolescents. Unsafe abortions due to unplanned pregnancies remain pervasive, contributing substantially to unmet needs for family planning service use in Ghana.

To meet women reproductive health care needs in rural Ghana, it is important to understand contextual demands for reproductive health care. Studies on contextual barriers to reproductive health care in Ghana exist. However, few studies have examined the concept of unmet maternity and reproductive care needs broadly in several heterogeneous rural districts in Ghana. Along single measures for unmet needs, one known study in Ghana presents an assessment of unmet needs for essential obstetric care services in rural Ghana (Baiden et al., 2006). Existing outcome measures at national and local levels for reproductive health care capture general measures such as supervised delivery coverage, antenatal and postnatal coverages and family planning acceptor levels. These broad indicators are insufficient to understand contextual drivers of unmet maternity and reproductive care needs in rural Ghana. Aside these, the adequacy of focused maternity care have minimally been investigated in Ghana based on WHO recommendations on focused maternity care.

This dissertation focuses on the understanding of unmet reproductive health care needs along a woman's life course in rural Ghana. The dissertation scope includes women in reproductive age (15-49 years). Methodologically, the concept of unmet need applied here is not based on an estimation of algorithm variables. Guided by the conceptual basis of unmet need, the dissertation examines Ghanaian context factors, social, environmental and economic arrangements and their role effects for unmet reproductive health care needs. It provides a segmentation of women experiences on the adequacy of maternity care received during their last birth. A further understanding of women's most crucial health needs across their productive and reproductive years is also provided. At the policy level, context findings from this dissertation will reflect shifting needs for patient-centered caring practices on reproductive health care in Ghana and across Sub-Saharan Africa (SSA). 


\subsection{Unmet need for reproductive health care}

\subsubsection{Unmet need concept and operational definitions}

In the 1980s, the concept of unmet need was predominantly applied in family planning literature. A woman has a met need for family planning if she has satisfied demand (adequate access and utilization) to meet her preference, timing and demands on family planning and other maternity care services. A woman is thus, referred as having an unmet need if her desired fertility needs (spacing and limiting) are not timely met. In the recent decade, several authors have applied the concept of unmet need to assess different healthcare needs patterns across general population groups (Agree \& Freedman, 2003; Black, Rabins, German, McCuire, \& Roca, 1997; Diamant et al., 2004; LaPlante, Kaye, Kang, \& Harrington, 2004; Newacheck, Hughes, Hung, Wong, \& Stoddard, 2000; Wells, Klap, Koike, \& Sherbourne, 2001). According to Sedgh et al (2007), four conditions must exist for a woman to be classified as having an unmet need; (i) the woman is in a marital or consensual union (ii) accounts of her non-pregnancy status, infecund and amenorrhea (simple terms fecund woman) (iii) does not want children in the period (at least in the next two years) (iv) and do not use any modern or traditional methods of family planning. A total of 15 survey items have been applied in DHS surveys based on several revisions of unmet need estimation for family planning. Follow up survey questions then assess the unintended/unwanted consequences of non-use and the women's abilities to meet or not meet their demand for child bearing.

From the above explanations, the unmet need concept for family planning has been long documented. This has never been the case for unmet need for other reproductive care components. For example, knowledge is still limited with regards to issues of unmet needs in relation to other reproductive components such as sexually transmitted infections and safe motherhood interventions. Study findings on barriers for maternity service provision and utilization are often referred to unmet need studies for maternity care. Other broad estimates of health outcome measures and determinants for maternity service provision and utilization are also often referred to as unmet need for maternity care. In this dissertation, the lack of a concise definition for unmet need for other components of reproductive care is acknowledged. Applying a conceptual basis of unmet need for family planning, this dissertation explores existing shortcomings that are related to unmet needs for reproductive care service provision and utilization. The focus is on the identification of context factors and their social, environmental and economic roles shaping reproductive health care behaviors. This paradigm is important in order to broaden the concept of unmet need beyond family 
planning, into all other components of reproductive health care along a woman's reproductive life cycle.

\subsubsection{Unmet need history ${ }^{1}$ and relevance ${ }^{2}$}

To provide evidence on women's desires for fertility regulation and family planning services across developing countries, the first set of Knowledge, Attitudes and Practices (KAP) study surveys were conducted in the early 1960s (Bradley \& Casterline, 2014). The aim for these KAP surveys was to estimate existing gaps in women's perceived knowledge, attitudes and their practices related to contraceptive use. This gap is referred to as the "KAP gap"(Casterline \& Sinding, 2000; Hauser, 1983). The measure of the "KAP gap" brought to the forth the hidden complexities of women unmet reproductive health needs. The difficulties in how to estimate women's unmet reproductive health needs also became apparent. Estimates of the "KAP gap" in these earlier surveys were conducted only among married women who wanted to stop child bearing, but were not using contraception. Other population sub-groups such as unmarried women, women desiring birth spacing and fecund women were unaccounted for in these earlier survey (Gilda Sedgh, Rubina Hussain, Akinrinola Bankole, \& Susheela Singh, 2007). Later, researchers including Westoff and Pebley extended the concept and theory behind KAP surveys, and renamed the concept "unmet need for family planning"(Westoff \& Ochoa, 1991; Westoff \& Pebley, 1981). After the emergence of the concept, several modifications concerning the algorithm of the measure were developed by Westoff and other colleagues using DHS data in the 1980s (Sedgh et al., 2007; Westoff \& Bankole, 1996). Factors such as a women's pregnancy state, infecund women, women's desire for birth spacing and breastfeeding mother's need for family planning were added to the model for estimating unmet need. Other unmet need proponents such Bongaarts and Dixon-Mueller (Bongaarts, 1991; Dixon-Mueller \& Germain, 1992) criticized Westoff estimation measures and called for a refinement to estimate unmet needs. A component of refinement advocated by Bongaarts and colleagues is the inclusion of birth spacing time periods for women (Sedgh et al., 2007). Others include the inclusion of views among non-regular and ineffective users of contraceptives, and those that use traditional methods of contraception (Dixon-Mueller \& Germain, 1992).

Other proponents advocated inclusion of measures such as pregnant and women with amenorrhea, women future fertility desires, and accounts of husbands needs and discordant fertility preferences among couples (Bankole \&

$1 \quad$ This section draws on Caterline \&Sinding 2000, Bradley et al 2014 \& Sedgh et al 2007. 
Ezeh, 1999; Gilda Sedgh, Rabina Hussain, Akinrinola Bankole, \& Susheela Singh, 2007). Measures for unmet needs among unmarried women (sexual active and have sexual intercourse one month prior to survey) were later developed by Westoff and Bankole in 1995, as part of DHS surveys conducted across 19 SSA countries (Sedgh et al., 2007).

As outlined by Sarah, Bradley and Casterline (2014) above, the concept of unmet need remains relevant based on three key reasons. First, unmet need estimates provide useful lessons for tracking national and global health goals. Second, unmet need seeks to provide a conceptual relationship between rising or declining population growths rates globally on the one hand, and the ability for couples to attain their satisfied demands for contraception. Last, appropriate unmet need estimation remains important to attract the needed investment for addressing inequalities in maternity and reproductive health services, particularly in developing countries.

\subsubsection{Key challenges confronting unmet needs}

The period of the Sustainable Development Goals (SDGs) provided the opportunity to address pervasive inequalities for maternity and reproductive service provision globally (Requejo et al., 2015). Improving inequality in the use of maternity and family planning services is pivotal to attain better health outcomes (Watkins, 2014). Reinforcing the link between equality of opportunity and outcomes for maternity and family planning is also important for attaining good health outcomes for women. In most developing countries, social norms and beliefs still govern ill-health. Among many low socio-economic women groups, policies that improve their social protection yield better health outcomes. To address some underlying causes for unmet needs, social norms that govern health seeking under some context must be understood. National polices and health system arrangements that provide for adequate funding are vital, particularly in countries without social health insurance policies. An amalgam of funding schemes and best practices in each country context remains paramount (Ensor \& Ronoh, 2005) for a long-term sustainability of family planning and maternity care services. Existing vertical programs in some developing countries must address indirect barriers to care access and utilization (Ensor \& Ronoh, 2005). Interventions that tackle reproductive health commodity and market inefficiencies (commodity shortages and social marketing/ franchising schemes), health infrastructure and skilled care for vulnerable groups need greater investments at national levels. Policy coherence at national, regional and global level will reinforce and improve key service indicators for women (Marmot et al., 2008). The post-Millennium Development Goals (MDGs) era provide a basis for monitoring major developments and health gains. For countries 
without vital registration systems and national data backbones, support is needed to provide these vital resources for improving reproductive health service delivery. This tracking must continue to provide lessons and best practices to improve family planning and maternity health services utilization among underserved populations.

\subsection{Trends in met and unmet reproductive care needs globally}

\subsubsection{Global and country met and unmet needs for reproductive care}

Globally, the burden of unmet need for family planning is high in SSA, compared with other regions of the world (see Figure 1.1). Unmet need for family planning is lowest in Europe and Central Asia compared with other regions (Figure 1.1). An estimated 35\% of women in Latin America and the Caribbean, $28 \%$ in Africa and 23\% in Asia cite health risks and side effects for not using contraception (Sedgh \& Hussain, 2014). Poor and inefficient health systems are known to characterize family planning and maternity care services delivery across regions such as Asia and Africa. In West Africa and South-Central Asia, male partner opposition to women's use of family planning accounts for high reported levels of unmet needs (30\% and 31\% respectively) in both regions (Sedgh \& Hussain, 2014).

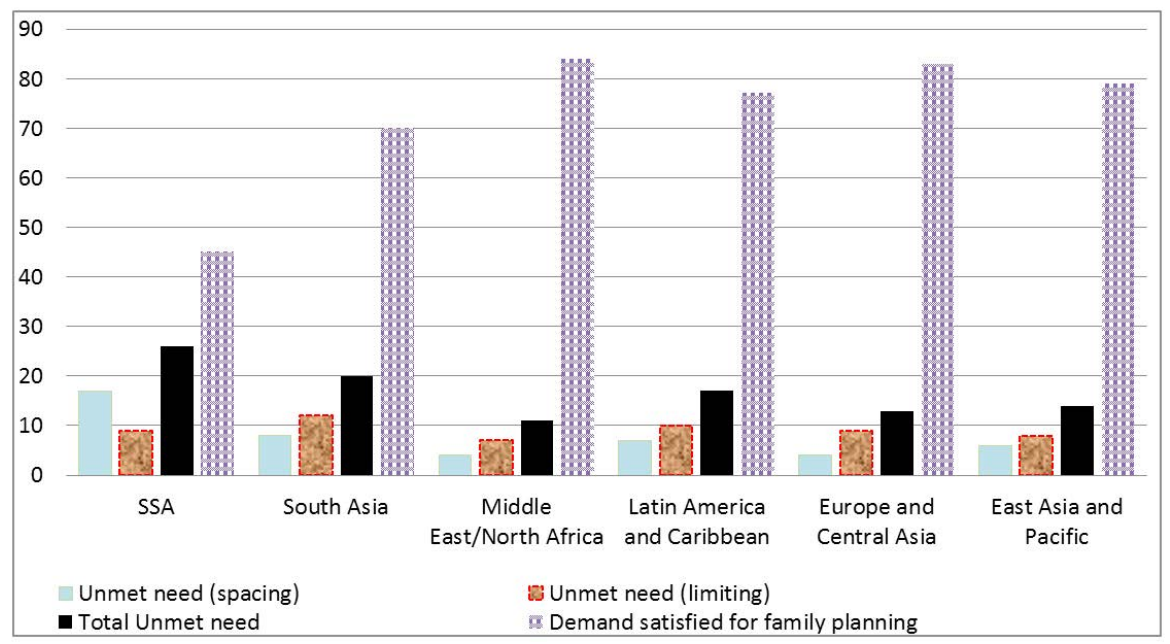

Figure 1.1: Unweighted means for global trends of unmet need for family planning. Source: Mills, Bos \& Emi, 2010

The WHO states that poor and inefficient health system designs accounts for poor patient-centered maternity and family planning care needs in most developing 
countries (WHO, 2012). Health systems are known to be weak in rural settings where a large share of the population resides in many developing countries.

Aside this, most services tend to be more physician-oriented in these stings already suffering from low skilled health workforce (ten Hoope-Bender et al., 2014; WHO, 2012). Evidence shows that health system changes in developing countries do little to increase skilled provider numbers for maternity care (Buchan \& Calman, 2004). Inadequate health infrastructure, and inadequate diagnostic supplies continue to impact the quality and levels of general healthcare delivery in developing countries (Watkins, 2014). Over the last decades, skilled providers of ANC and facility-based birthing have seen immense improvements across many countries in West Africa (see Figure 1.2).

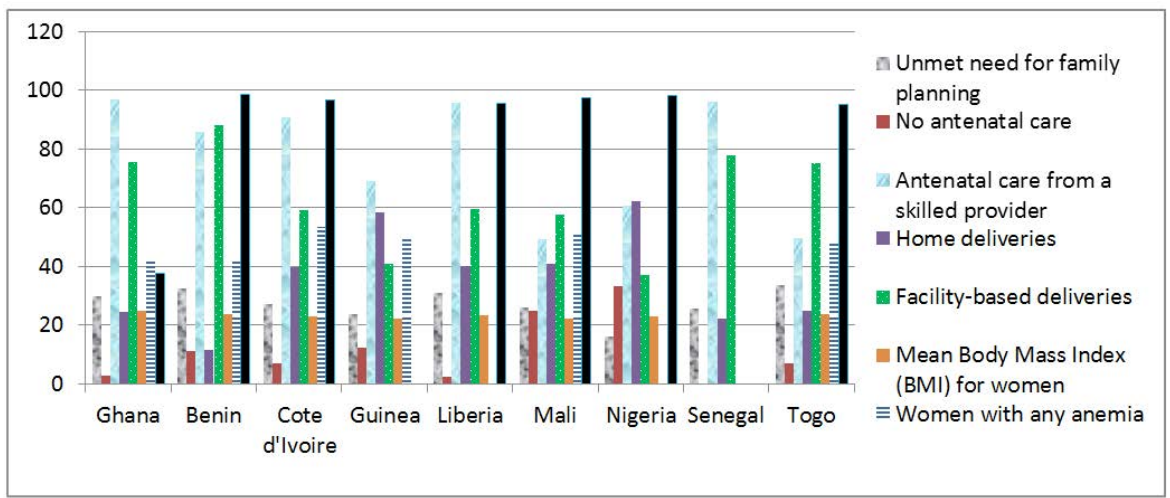

Figure 1.2: Met and unmet care demands across selected West African Countries from DHS surveys (20112014). Source: DHS Program STAT compiler online (www.statcompiler/en.com).

As can be seen from Figure 1.2, eight countries (Benin, Cote d'Ivoire, Guinea, Liberia, Mali, Nigeria, Senegal and Togo) had more women (>90\%) reporting no health insurance for accessing care during pre/postpartum periods. A comparison of six countries in West Africa shows unmet needs remain high (see Figure 1.3). In addition, two West African countries, Nigeria and Guinea are observed to have recorded lower facility-based deliveries ( $<50 \%$ of pregnant women) as evidenced from Figure 1.2. This data also show women in Nigeria and Mali had a high propensity of not receiving ANC (> $20 \%$ of pregnant women). 


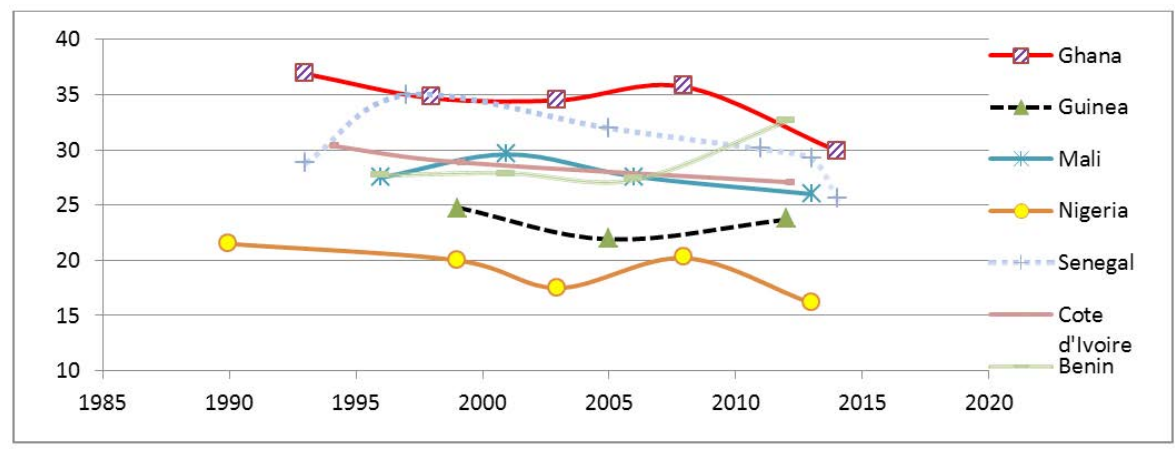

Figure 1.3: Unweighted percentage of married or in union women with unmet need for family planning in selected West African countries from 1990-2014. Source: DHS STAT compiler online (www.statcompiler/ en.com).

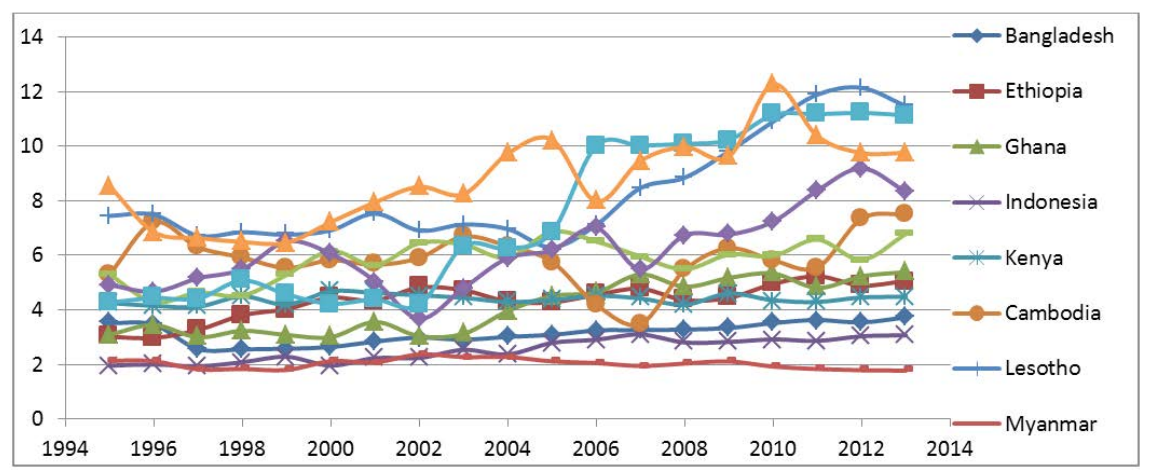

Figure 1.4: Total health expenditures (\% of GDP) in selected developing countries Source: World Bank Development Indicators assessed ( http://data.worldbank.org/data-catalog/world-development-indicators). All data constructed is unweighted. Data was assessed 31 March, 2016.

Social health insurance ownership improves healthcare access for many population groups, although national health insurance coverages remain low in many SSA countries. Across most developing countries, government expenditure on maternal health as a proportion of total government expenditure ranges from as low as $2 \%$ in Burundi (APP, 2010). Countries such as Kenya, Ghana and Rwanda however made significant allocations to national budgets since 2009 to improve in maternal health interventions (APP, 2010). 


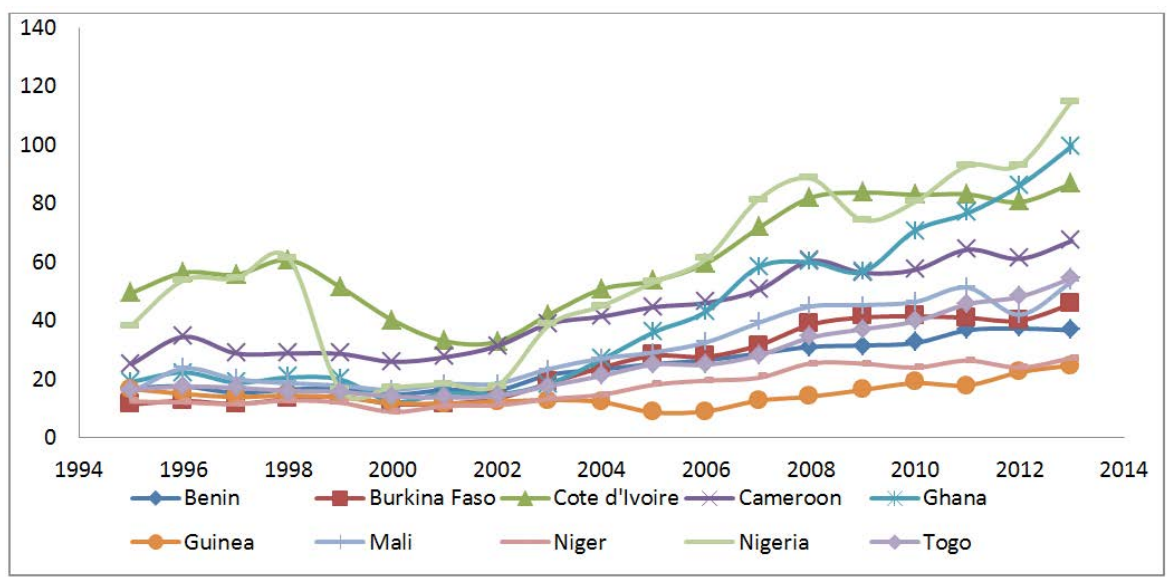

Figure 1.5: Health expenditure per capita (current US\$) in selected West African Countries. Source: World Bank Development indicators assessed from (http://data.worldbank.org/data-catalog/world-developmentindicators.) . Data covers the provision of health services (preventive and curative), family planning activities, nutrition activities, and emergency aid designated for health. Amounts were estimated based on prevailing U.S. dollars equivalents Data was assessed 31 March, 2016.

Most African countries do not apply economic evaluation to reproductive, maternal and new-born interventions as stated by African Progress Panel 2010 report. Furthermore, out-of-pocket expenditures on reproductive and maternity care services remain high in these countries. This has the potential to negatively impact access to family planning and maternity care services among vulnerable population groups.

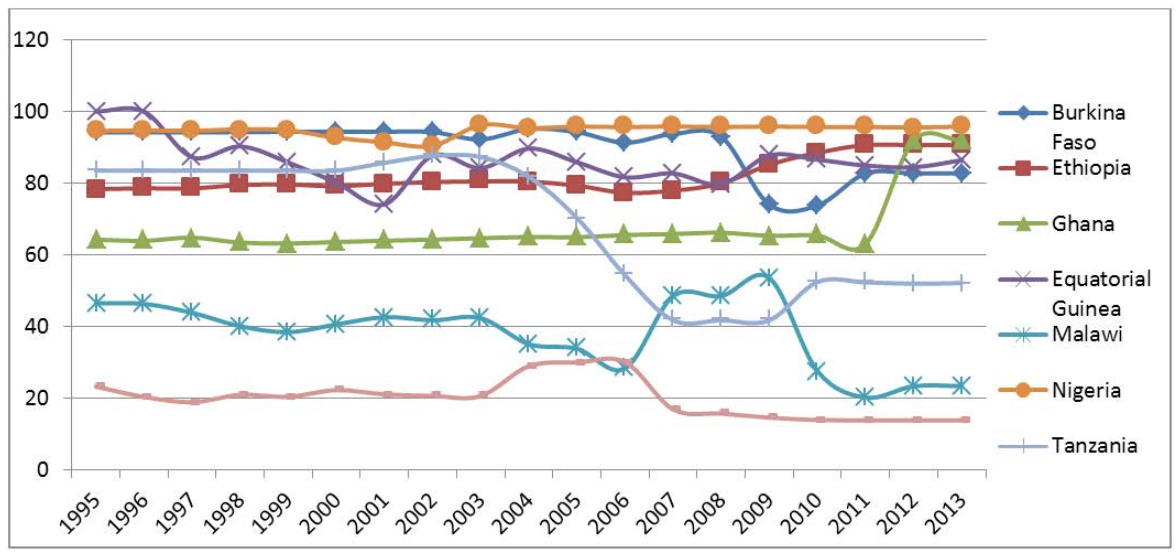

Figure 1.6: Out-of-pocket health expenditure (\% of private expenditure on health) trends across selected African countries. Source: World Bank World Development Indicators data online (http://data.worldbank.org/datacatalog/world-development-indicators). All percentages are unweighted. 
Additionally, World Bank data on out-of-pocket health expenditures across Africa show high rates ( most countries spend $>50 \%$ of private expenditures on health) as presented in Figure 1.6.

From Figure 1.6, it can been seen that only South Africa has a lower rate of outof-pocket payments $(<30 \%)$ in 2013 than in 1995. Five countries, Ghana, Nigeria, Tanzania, Equatorial Guinea and Burkina Faso show high levels $(>80 \%)$ of outof-pocket expenditures in 2013 (see Figure 1.6). Across countries in West Africa, residence type (rural/urban) and socio-demographics influence met and unmet maternity and family planning care service levels (Adongo et al., 1997; Ayanore \& Aryee, 2015). Socio-cultural norms and practices resulting in a discontinuation of services use, economic constraints, and longer travel distance to access services and low education levels of women were found to be key predictors of service utilization for most maternity care services (Ganle, 2014; OlaOlorun, Seme, Otupiri, Ogunjuyigbe, \& Tsui, 2016). Furthermore, poor male spousal support is known to dictate women reproductive behaviors among many countries in Africa. A review of studies across developing countries (Say \& Raine, 2007) show gender inequality estimations are important predictors of reproductive health services use by women. Assuring adequate numbers for skilled professional care remains a surmountable challenge in most countries in SSA. Across most countries in SSA, Traditional Birth Attendants (TBAs) play a major role in delivering maternity care service. In countries, such as Nigeria, Zambia and Uganda, TBAs remain instrumental in helping women meet maternity care demands (Adeniran, Ogunnowo, Wright, \& Odusanya, 2015; Sialubanje, Massar, Hamer, \& Ruiter, 2015; Turinawe et al., 2016). A review of health system interventions in Ethiopia that deployed health extension workers (HEWs) showed remarkably diffrences in care coverages for family planning and ANC services (Koblinsky et al., 2010; Medhanyie et al., 2012). However, later evidence of this Ethiopian model found that this cadre of health care workers contribution to overall maternal care was limited (Admassie, Abebaw, \& Woldemichael, 2009). Globally, assessing unmet needs across different population's context is important to address existing unmet needs for maternity and family planning service utilization. The importance of context for meeting broad reproductive care needs cannot be overemphasized. Part of the challenge in addressing safe reproductive care needs for women is the recognition of women shifting reproductive preferences in any context. This, together with health systems that provide services in line with social norms could upscale reproductive services use.

In Ghana, socio-cultural and demographic determinants of maternal and family planning use have been widely documented over the last decades (Adongo et al., 1997; Atinga \& Baku, 2013; Josephine Borghi et al., 2003; Filippi et al., 2006; Perkins et al., 2009; Sophie Witter et al., 2007). Evidence from the 2014 
Ghana Demographic Health Survey (GDHS) report suggest that total demand for family planning is high among women within the ages 15-19 (69\%) and low for women between $45-49$ years ( $33 \%$ ). In Ghana, unmet need for family planning is comparatively high at $29.9 \%$ relative to other countries in West Africa (see Figure 1.3). The national percentage for women demand satisfied for family planning is also low at $47 \%$ (Figure 1.6). Furthermore, recent data from the World Bank shows that out-of-pocket payments increased sharply between 2011 and 2013 in Ghana compared to other West African countries (Figure 1.6). Other studies in Ghana point to social and health system constraints for maternity and reproductive care services (Addai, 1998; Asamoah, Agardh, Pettersson, \& Östergren, 2014; AtunahJay, Pettingell, Ohene, Michael Oakes, \& Borowsky, 2013; Lambon-Quayefio \& Owoo, 2014). In Figure 1.7, sharp increases for skilled provider care, antenatal visitations and demand satisfied for family planning are observed.

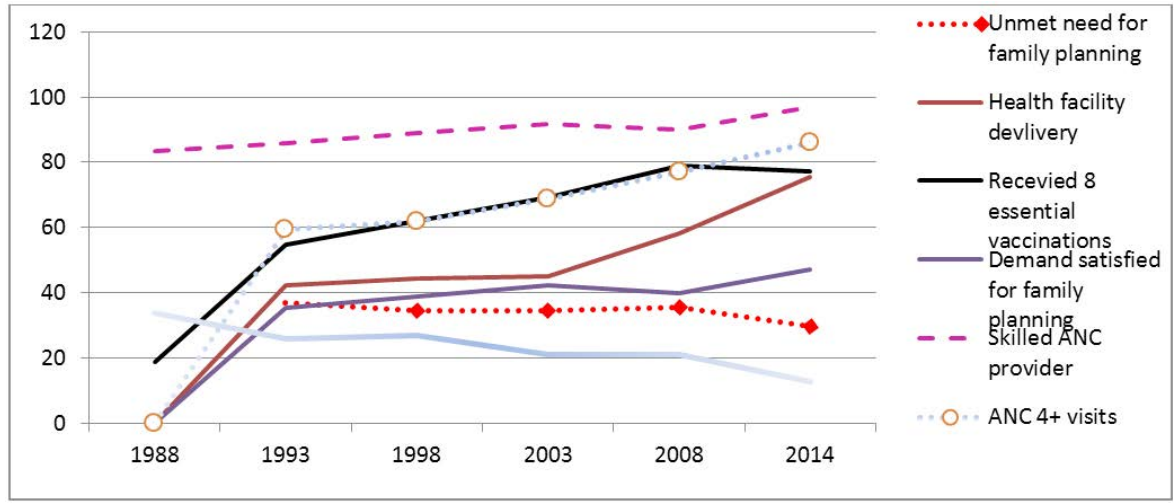

Figure 1.7: Maternity care trends across selected indicators in Ghana from 1988-2014. Data extracted from DHS Figure 1.7: Maternity care trends across selected indicators in Ghana from 1988-2014. Data extracted from DHS Program; STAT compiler online (http://www.statcompiler.com/en/index.html).

To reduce unmet needs for maternity and family planning service in Ghana and SSA, policies and strategies that address patient-centered care needs are important. Patient-centered care is defined as care delivered to meet patient's value preferences and quality context care needs (Luxford, Safran, \& Delbanco, 2011). The provision of patient centred care however requires reliable country context data and strategies that work in each context among most vulnerable population groups globally.

\subsection{Aim and objectives of the dissertation}

This dissertation aims to increase our understanding of unmet maternity and reproductive health care service needs along a woman's life course in rural 
Ghana. Four principle objectives underpin this dissertation. The four objectives in summary assess the adequacy of outcome measures, focused maternity and reproductive care and stakeholder's view on attaining adequate motherhood health outcomes in rural Ghana. The four principle objectives that underline the basis of this dissertation are outlined below:

First, this dissertation explores the adequacy of outcome measures and determinants in West Africa for unmet reproductive health care needs. The assessment of outcome measures and determinants across West Africa will enable an understanding of Ghana's own context relative to other countries in West Africa. Similarly, context similarities between Ghana and other countries in West Africa make it imperative to investigate in countries West Africa. Understanding the adequacy of outcome measures on reproductive health care in West Africa is necessary to provide policy makers, researchers and health policy implementers with information to compare progress across the sub-regions. Adequate knowledge and application of outcome measures on reproductive care will assist in addressing disparities in maternity and reproductive care use among women in Ghana. Overall, the critical examination of local context measures and determinants is important to identify what targets drive reproductive health care provision and utilization in Ghana (Asante, Chikwama, Daniels, \& Armar-Klemesu, 2007). An investigation in this direction can provide evidence for policies to improve quality patient-centered standards on reproductive care. For health researchers, such investigation is important because it can outline the strengths and weaknesses of defining local context factors for measuring the progress regarding women health outcomes.

Second, although, successive governments in Ghana prioritized stakeholder involvement in delivering health services, existing studies have not fully examined stakeholders' perspectives on addressing shortcomings in service utilization. Evidence on top-down uncoordinated planning among health policy stakeholders exists (MOH, 2008), with little evidence on how these decisionmaking approaches could be improved. Additionally, the few studies in Ghana applied a mix of qualitative approaches to better understand maternity care barriers (Halley Crissman et al., 2013; Lori et al., 2012; Pell et al., 2013). By applying a combination of qualitative and quantitative approaches, this dissertation triangulates the views of stakeholders at the service provision and beneficiary level. Such analysis provides an in-depth understanding of stakeholders' views on maternity shortcomings in rural Ghana.

In addition, it provides an opportunity to examine stakeholder experiences, expectations and decision making processes relevant to the continuous improvement of reproductive health outcomes in rural Ghana. 
Third, delivering patient-centered reproductive health care services requires multiple stakeholder involvement. Stakeholder's understanding of local context strategies that improve reproductive care provision may enhance reproductive service utilization. Facility, community, and district level evidence on stakeholder engagements on care provision is growing. Nonetheless, bottlenecks exist on how these stakeholder views could further enhance quality of reproductive care in Ghana. Previous studies applying DHS data to assess barriers to contraception use in rural Ghana have largely focused on the perspectives of women within the reproductive age (18-49 years). To capture rural contraceptive needs among young adolescent women, this dissertation provides evidence of contraceptive demands and use among young mothers (18-28 years) in rural areas in Ghana. It is important to focus on this age group as many young adolescents enter into motherhood with reported unmet maternity and reproductive service needs. This dissertation includes this age group (18-28 years) in its analysis with the aim to capture young women expressions of met and unmet needs along the continuum of maternity and reproductive care. Evidence of young adolescent women examined here could provide health care providers and health policy actors with better targeting reproductive care strategies for most adolescents in transition into motherhood in most rural Ghanaian settings.

Lastly, existing empirical evidence on barriers to maternity and reproductive care utilization assesses single group trends. Few existing studies provide a segmentation of women's met and unmet care needs for maternity and reproductive care needs. Such segmentation remains important to understand the standards of unmet needs for maternity and reproductive health care. Extending the concept of unmet needs to maternity care, a concept rarely discussed outside the domain of reproductive health, distinguishes this dissertation from many studies in Ghana. The evidence on women's views on the levels of care adequacy (adequate, less and least) from previous birth episodes is important to identify and target vulnerable women populations in need. The identification of reported adequate care received from such segmentations is also important to inform health program planners and implementers what context factors affect these specific populations groups. This is important for improving maternity and family planning outcomes.

Although this dissertation focuses on rural Ghana, its relevance has important policy lessons for countries in SSA. As outlined above, many countries in SSA have incessant high unmet reproductive health need levels. Findings from this dissertation provide countries in SSA strategies for delivering patient-centered maternity and reproductive care needs. In addition, policy actions on the roles of stakeholder views, social health insurance, focused counselling, and clinic level arrangements will further enhance reproductive service quality and satisfaction among rural populations. The emphasis in this dissertation on the need for local 
context outcome measures is important to identify gaps in reproductive health care. To unearth evidence related to the knowledge gaps outlined above, this dissertation employs a broad perspective on reproductive care and maternity care. It challenges the adequacy of existing outcome measures in improving women's chances of maternity and reproductive care utilization in West Africa and SSA. In addition, it triangulates the stakeholders' experiences and views on reproductive and maternity care utilization, which distinguishes this dissertation from previous studies in Ghana. The study findings are of interest due to the increasing demand for evidence based knowledge to improve maternity outcomes in Ghana. Evidence from stakeholder findings provides a baseline for evaluating stakeholder support on maternity and reproductive care needs in Ghana. The dissertation also focuses on generating new empirical evidence and understanding of existing policy trends to deliver patient-centered maternity and reproductive care in Ghana. To achieve these aims, three main research questions and data sources are outlined;

Research question 1: What is the adequacy of outcome measures and determinants used to assess women unmet reproductive health needs across West Africa?

Individual context factors, gender, and social roles interact and shape reproductive health behaviors across most population groups in West Africa (Brazier et al., 2009; Eliason et al., 2013c). To provide an understanding of the adequacy of outcome measures and determinants in reproductive care, a systematic review of the evidence on outcome measures and determinants is undertaken in West Africa. Considering context similarities and reported low uptake of contraceptive use across West Africa (Jacqueline Darroch \& Singh, 2013; Loaiza \& Blake, 2010), it is important to better understand outcomes measures and determinants applied for assessing reproductive health outcomes across this region. Three outcome measures are evaluated in this review; contraceptive use, obstetric care, and ANC. No such comprehensive review has been undertaken in West Africa. Databases searched include PubMed, Econchapter s, CINAHL, Psych INFO, Science Direct, Embase and Biomed. In the PubMed search, MsSH term categories are applied across all key words to ensure that all relevant publications are extracted for the review. The review attempts to provide answers to the adequacy of outcome measures and determinants applied in previous studies in relation to unmet maternal and family planning needs in West Africa. Evidence from this research question challenges the adequacy of outcome measures and determinants of service use. The need for a review of the development of contextspecific measures is highlighted and advocated in this research question of the dissertation. 
Research question 2: What views on maternity care shortcoming stakeholders' hold in service provision and utilization in rural Ghana?

Stakeholders' views in Ghana on maternity care shortcomings cites poor obstetric care, informal payments, poor allocation of skilled providers and unfriendly health provider attitude as factors impeding service provision and utilization (Arthur, 2012; Atinga \& Baku, 2013; Josephine Borghi et al., 2003; Perkins et al., 2009). Evidence from other studies show many barriers for maternity care seeking, which are related to providers, health system features and user characteristics (Addai, 1998; Asamoah et al., 2014; Atinga \& Baku, 2013; Atunah-Jay et al., 2013; Josephine Borghi et al., 2003; Filippi et al., 2006; Ganle, 2014; Ganle et al., 2015; Lambon-Quayefio \& Owoo, 2014; Perkins et al., 2009; Sophie Witter et al., 2007). Policy directives in the past decades have prioritized private health actor's role in health service delivery. This provides an opportunity for mapping stakeholder interests across several levels in service delivery. Given the potential effects of effective stakeholder collaboration for delivering effective maternity health services, few studies have investigated policy gaps and priority settings to improve safe motherhood interventions in Ghana (Madi et al., 2007; Rudan et al., 2010; Witter \& Adjei, 2007; Sophie Witter et al., 2007). Most studies on shortcomings for maternity care analyze users of maternal care as a single group, and provide trends over a specific period. None of these studies provides a segmentation of women's met and unmet care needs based on WHO recommended care components (WHO, 2002). Such segmentation is important to understand the standards of care met and unmet in previous DHS reports. In addition, no known previous study triangulated the evidence on shortcomings for maternity care in Ghana across rural districts to unearth key stakeholder perspectives for improving maternity and reproductive care in Ghana.

To answer this research question, data from two sources are used. First, to understand the components of maternity care women receive in Ghana based on WHO recommendations, the 2008 GDHS dataset was used. The content of the questionnaire in the 2008 GDHS study was based on model questionnaires developed by MEASURE DHS and the 2003 GDHS questionnaire. The GDHS is a nationally representative survey, implemented with the technical support from ICF Macro and MEASURE DHS program. Women aged $15+$ years were eligible to be interviewed if present in the household the night before the interview. In total, 4,916 eligible women were interviewed in the survey. This was made up of 2,162 urban and 2,754 rural eligible women. From them, 2,147 women (763 urban and 1,384 rural women) within reproductive age (aged 15-49 years) are included in the analysis for this dissertation because they reported childbirths within the last 5 years. Second, additional primary data were collected using a mix of qualitative 
techniques to assess stakeholder views on maternity care shortcomings in three rural districts in Upper East Region of Ghana; Bongo, Talensi and Nabdam districts. Data were collected using ethnography among three key stakeholder groups: users of services (women) with recent births $(n=90)$, healthcare providers $(n=16)$ and policy actors $(n=6)$. Focus group discussion (FGDs) and in-depth interviews (IDIs) were used to collect data between March and May 2015. Interview guides on shortcomings for maternity care contained questions on four key domains; service provision, lifesaving skills and knowledge, quality, cost and satisfaction and overall system level shortcomings on maternity care. Findings from this study outline social, economic and health system lessons to improve the utilization of prenatal, postnatal and medication care in Ghana.

Research question 3: What factors account for unmet reproductive care needs in rural Ghana?

The complexity of sexuality and womanhood is mediated by other factors such as gender interest and roles, male cultural dominance, and gender inequities in some developing countries (Blanc, 2001; Halley Crissman, Adanu, \& Harlow, 2012; Gerein, Green, \& Pearson, 2006). Evidence also shows that traditional reproductive approaches are insufficient to improve reproductive outcomes (Malhotra \& Mehra, 1999). In recognition of these effects on reproductive wellbeing, sexual health emerged as a new paradigm to improve sexuality and sexual health outcomes for women (Halley Crissman et al., 2012; McIntosh \& Finkle, 1995). Most studies on sexuality and reproductive health in Ghana are limited to contraceptive use, while few studies have examined decision making processes, sexuality choices and support mechanisms for reproductive health among women. This dissertation investigates formal and informal decision processes and social underlying prepositions that affect reproductive health service use in rural Ghana.

Both qualitative and quantitative data are triangulated across four rural districts in Ghana to answer this research question: Garu-Tempane, Bongo, Talensi and Nabdam. First, a community based descriptive cross-sectional design approach was applied where a standardized questionnaire enabled the collection of data among 720 young women (18-28 years) in the Garu-Tempane district of Ghana. FGD and IDI guides was applied where data on the view of two groups; women and men $(n=40)$ within the reproductive age and midwives $(n=3)$ was collected. The standardized questionnaire and interview guides provided data on women, men and midwives views of contraceptive use, access on demand, and future use intentions. All data was collected between February to March 2012. Second, to establish stakeholder perspectives and corroborate evidence 
on unmet reproductive health services needs in rural Ghana, an ethnography design was applied. IDIs and FGDs interview guides collected data among three groups; users of services (women), health care providers and policy actors. Data collection was undertaken in districts where our maternity care shortcoming study was conducted (see research question 2 above). Women users $(n=48)$, health care providers $(n=14)$ and policy actors $(n=4)$ were interviewed to gather qualitative evidence on service provision, utilization and barriers for adequate care services in rural Ghana. The evidence espoused for this research question provided a basis for the debate on the nexus between meeting culturally sensitive care and unmet sexual needs among women in male dominated environments. It reflects health system barriers that need policy reformation and coordination to improve inequalities for services delivery among vulnerable populations groups in Ghana.

\subsection{Outline of the dissertation}

Chapter 1 of this dissertation (this chapter) sets out a general overview of the concepts and measures of unmet reproductive health care needs. It explores global, regional and country level unmet reproductive health need concerns in literature. Chapter 1 concludes with the examination of this dissertation aim, and defines clearly the dissertation objectives and research questions. The dissertation is then subsequently divided into three main sections. Section 1 comprises of Chapter 2, which reports on the results of the systematic review of the adequacy of outcome measures and determinants for unmet reproductive health needs applied in previous studies in West Africa. Section 2 consists of Chapter 3 and Chapter 4. Chapter 3examines the adequacy of focused maternity care across all regions in Ghana whiles Chapter 4 assesses stakeholder perspectives on maternity care shortcomings in three rural Ghanaian districts. In section 3, Chapter 5 and Chapter 6 are presented. Chapter 5 assesses the importance of context-specific associations for women (18-49 years) contraceptive use in a rural Ghanaian district.

Chapter 6 outlines stakeholder experiences, expectations and decision making on reproductive care in rural Ghana. A general discussion of the key dissertation findings is provided in Chapter 7. 


\section{CHAPTER 2 \\ Unmet reproductive health needs among women in some West African Countries: A systematic review of outcome measures and determinants}

Draws upon published work:

Ayanore, MA., Pavlova, M., Groot, W. (2016). Unmet reproductive health needs among women in some West African countries: a systematic review of outcome measures and determinants. Reproductive Health, 13(1):5. DOI: 10.1186/s12978015-0104-x 


\section{Abstract}

Introduction: Identifying relevant measures of women's reproductive health needs is critical to improve women's chances of service utilization. The chapter aims to systematically review and analyze the adequacy of outcome measures and determinants applied in previous studies for assessing women reproductive health needs across West Africa.

Methods: Evidence on outcomes and determinants of unmet reproductive health needs among women of childbearing age in diverse multicultural, religious, and ethnic settings in West African countries was systematically reviewed. The review included recent English language publications (from January 2009 - March 2014). Clinical studies particularly on obstetric care services and reproductive services in relation to HIV/AIDS were excluded. Outcomes and determinants were assessed and defined at three main levels; contraceptive use, obstetric care, and ANC utilization.

Results: Results show increasing unmet need for women's reproductive health needs. Socio-cultural norms and practices resulting in discontinuation of service use, economic constraints, travel distance to access services and low education levels of women were found to be key predictors of service utilization for contraception, antenatal and obstetric care services. Outcome measures were mainly assessed based on service utilization, satisfaction, cost, and quality of services available as core measures across the three levels assessed in this review.

Conclusions: Evidence from this review indicates that currently applied measures of women's reproductive health needs might be inadequate in attaining best maternal outcomes since they appear rather broad. More support and research for developing and advancing context-related measures may help to improve women's maternal health. 


\subsection{Introduction}

Globally, more than half a million women aged 15-49 years die annually from preventable pregnancy-related complications (AbouZahr \& Wardlaw, 2001; WHO, 2011) Women in developing countries have a 1 in 26 chance of dying from pregnancy and abortion compared to 1 in 9400 in Europe (Bremner et al., 2010). One of the factors associated with this outcome is the unmet health need for contraception and reproductive health care. Unmet reproductive health needs exist if there is a gap between a perceived need and the current available options to satisfy the need (see Chapter 1). This chapter focuses on three levels of unmet reproductive health needs: contraceptive service use, obstetric care, and ANC care utilization.

Contraceptive prevalence rates (CPR) globally for women aged 15-49 years, married or in a union rose from $58.4 \%$ in 1994 to 63.6\% in 2012. Yet, an estimated 222 million women still fall within the current unmet needs estimates for contraceptives, with $90 \%$ of these women currently in the developing world (Jacobstein, Curtis, Spieler, \& Radloff, 2013; Speizer, Calhoun, Hoke, \& Sengupta, 2013; WHO, 2011). As a result, one in five pregnancies is unintended (Glasier, Gülmezoglu, Schmid, Moreno, \& Van Look, 2006).

Women often resort to an abortion when faced with an unintended pregnancy (Grimes et al., 2006). These women are said to have an unmet need for contraception. It is also evidenced that the unmet need for ANC decreased in developing countries in the period 2000-2012 (Singh \& Darroch, 2012). Thus, despite the global health problems, family planning and antenatal service uptake have improved over the decades in developing countries (Gakidou \& Vayena, 2007). Nevertheless, more effort is needed to change the results in SSA.

SSA is evidenced to have the highest percentage of women with unmet reproductive health needs (see chapter 1). Also, SSA accounts for $24 \%$ of the global disease burden whiles only $3 \%$ of the world skilled health workforce come from this region (Jacqueline Darroch \& Singh, 2013). As evidenced in the literature, long travel distances to the nearest health facility, poor access and poor quality of maternal and newborn care provide a low probability of mother and child survival especially among rural women in SSA (Dogba et al., 2011).

This chapter addresses the unmet reproductive health needs in West Africa as this region has one of the poorest maternal and reproductive health indices in SSA (WHO, 2011). The situation is even worsening with an ever surge in population growth over the last decade (Bremner et al., 2010). In 2012 alone, 222 million women in developing countries were reported to have unmet need for modern contraception (Singh \& Darroch, 2012). This level of unmet need decreased in 
every sub-region between 2003 and 2012, but remains high in West Africa (74\%) compared to East Africa (54\%) (Jacqueline Darroch \& Singh, 2013). Additionally, West Africa has lower levels of modern contraceptive use ( $\leq 26 \%)$ compared with the higher levels of use (46-66\%) recorded in East and Middle Africa respectively (Jacqueline Darroch \& Singh, 2013). Since the review covers some West African countries, context-related similarities and differences should be acknowledged. Population policies to reverse the rising population growth rates have existed for the past decades in all countries in West Africa (Bloom, Sachs, Collier, \& Udry, 1998; Cleland et al., 2006; Jamal \& Weeks, 1988). However, much remains to be improved on ripping the demographic dividends of fertility declines in these countries (Cleland et al., 2006). Reviews across West Africa show low uptake of contraceptives due to reported contraceptive failures (Jacqueline Darroch \& Singh, 2013; Loaiza \& Blake, 2010) while religion, gendered interest, and social parity desires have also been long documented to work against family planning interventions (Brazier et al., 2009; Izugbara, Ibisomi, Ezeh, \& Mandara, 2010; Izugbara \& Ezeh, 2010). The situation of reproductive health services is especially challenging in countries recovering from civil war and instability, such as Senegal, Sierra Leone among others, while countries with political stability and continuous economic growth, like Burkina Faso, Ghana and Nigeria, have achieved political commitment and progress in improving these services.

Comparative evidence provided by the United Family Planning Population Agency (UNFPA, 2010) show that Benin, Burkina Faso, Mali, Nigeria, and Senegal witnessed significant positive changes in the levels of unmet needs over the last decade (Loaiza \& Blake, 2010). For example, Nigeria recorded a high percentage point change (19.5\%) between 2003 and 2008 for unmet need levels. Burkina Faso, Benin and Mali recorded percent changes of 11.6\% (1999-2003), 9.9\% (2001$2006)$ and $9.5 \%$ (2001-2006) respectively. However, no country was evidenced to have significantly improved its contraceptive use rates, although Burkina Faso and Nigeria were estimated to have made positive changes on contraceptive prevalence levels. Burkina Faso recorded a percentage point change of 16\% from 1999-2003 while Nigeria is evidenced to have a $15.9 \%$ improvement between 2003-2008 (Loaiza \& Blake, 2010). Overall, in West Africa, individual context factors such as gender and social roles interact and shape reproductive health behaviors across all population groups (Bove, Vala-Haynes, \& Valeggia, 2012; Brazier et al., 2009; Eliason et al., 2013; Izugbara \& Ezeh, 2010). Wealth, education, residence type, and age disparities however remain the greatest influencing factors introducing disparity in any context (Jacqueline Darroch \& Singh, 2013; Kirk \& Pillet, 1998; Loaiza \& Blake, 2010). Overall, policy implementations aimed at improving reproductive health outcomes vary from country to country depending on the level of accountability and transparency, economic growth, good governance 
principles, and social capital of the citizenry (Jacqueline Darroch \& Singh, 2013; Loaiza \& Blake, 2010). The fundamental recognition is that no single policy intervention is sufficient in any country context to make huge improvements in maternal reproductive health outcomes. The identification of outcome measures and determinants of women's reproductive health needs, which is the focus of this review, may help improve broader consensus on women's reproductive needs for greater health and economic outcomes.

The assessment of outcome measures and determinants applied from previous studies in this chapter will provide evidence on the adequacy of outcome measures and determinants applied in previous studies in relation to unmet reproductive health needs. This can be used to address policy and research gaps related to maternal health in this region. No such comprehensive review has been undertaken in West Africa. The review will provide further impetus for redoubling of commitments towards improving maternal health outcomes in SSA.

\subsection{Methods}

To assess outcome measures and determinants of unmet reproductive health needs among women in West Africa, a systematic review of recent publications in the area was carried out in 2014 following the Cochrane review protocols (Higgins \& Green, 2008). 


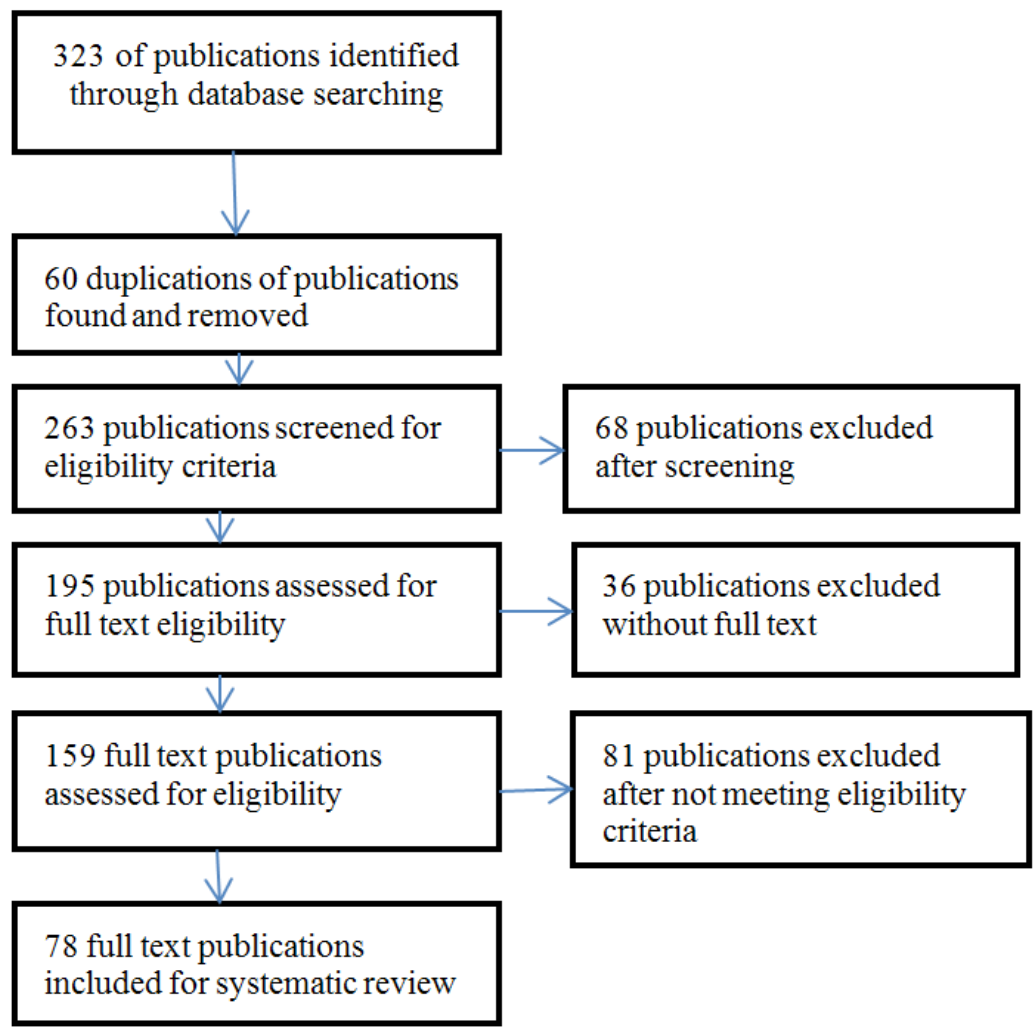

\section{Box 2.1: Main key words applied in the literature search for all databases}

Unmet need OR KAP Gap OR missed opportunities OR unattained health status OR never met needs OR never attained need OR not attained needs AND reproduction OR reproductive status OR reproductive system OR reproductive health AND contraceptive methods OR contraceptive method OR ANC services OR antenatal services OR abortion care OR contraceptive use OR obstetric care OR inhibition of fertilization OR birth control OR female contraception AND outcomeassessment OR outcome assessments OR measure outcomes OR predictors OR influencing factors OR evaluator factors OR study outcomes OR study outcome OR predictors AND determinant OR factor, OR factors AND West Africa* OR SSA OR developing country AND women OR woman OR girl OR girls.

* MsSH term for West Africa was applied. This query included all the under listed West African countries in the Search: Benin; Burkina Faso; Cote d'Ivoire; Gambia; Ghana; Guinea; Guinea-Bissau; Liberia; Mali; Mauritania; Niger; Nigeria; Senegal; Sierra Leone; and Togo. 


\subsection{Results}

A total of 323 publications were identified during the systematic search. This initial list included publications for 14 West African countries: Nigeria (103), Ghana (96), Senegal (23), Benin (17), Togo (9), Mali (21), Liberia (6), Sierra Leon (3), Cote D’ivoire (7), Gambia (8), Burkina Faso (22), Guinea (4) Mauritania (2) and Niger (2). No publication was found for Guinea Bissau. After controlling for the inclusion and exclusion criteria, a total of 78 publications across only seven countries were found and analyzed. An overview of the filtration process of articles reviewed is provided in Figure 2.1.

\subsubsection{General characteristics of reviewed publications}

Publications from only seven West African countries out of the fourteen assessed were included in the review. A total of 77 articles and 2 reports published in the period January, 2009 - March 2014 were analyzed. The majority of the articles were from Nigeria $(\mathrm{N}=39)$, followed by Ghana $(\mathrm{N}=23)$, Burkina Faso $(\mathrm{N}=8)$ and Mali $(\mathrm{N}=4)$, with only one article from Benin, Sierra Leone and Senegal respectively. The two reports were large studies based on the DHS that had investigated issues of reproductive health needs across low-income countries. The reports contained information from country-specific publications that did not appear during the search. Most studies were conducted in both urban and rural areas. The rest of the studies were carried out in either urban or rural areas only. The majority $(\mathrm{N}=52)$ were original studies that collected own data. Few articles $(\mathrm{N}=26)$ were based on the DHS data conducted across some countries. Two thirds of the studies had a descriptive aim whiles the rest had predictability aim, or had no clear objectives. Details about the study designs applied in the publications reviewed and the quality of these designs can be found in the appendix Table section.

Overall, a cross-sectional design was applied in most of the studies, with a variety of investigative modes and population-based surveillance designs. Different instruments to collect qualitative and/or quantitative data were applied. Five articles had secondary data reviews in addition to other techniques. Verbal narratives and use of observation checklist was also detected in three studies. Most studies focused on specific population groups (e.g. rural areas, urban areas or towns/cities). Only two articles reported country-wide studies. 
A high proportion of the articles had sample sizes less than 500 respondents $(\mathrm{N}=41)$ or more than 2000 respondents $(\mathrm{N}=31)$. Few studies had 500 to 2000 respondents.

\subsubsection{Specificity of outcome measures in reviewed publications}

Table 2.1 illustrates the outcome measures for three types of reproductive health services reported: Antenatal cre (ANC), contraceptive services and obstetric care. Three themes of outcome measures were distinguished: clinical, economic/geographical and patient-reported outcomes. Clinical based outcomes of ANC varied and included quality of service, satisfied demand, out-of-pocket payments, antenatal visits per woman and level of service utilization.

Contraceptive utilization outcomes at the clinical level included the existence of widespread contraceptive shortages coupled with limited choices for contraceptive use by potential users. Unintended pregnancies were widely observed with service constraints and provider attitudes resulting from scolding and intimidation of the clients by providers. At the clinical level, obstetric care was assessed on critical danger signs observed from pregnancy to birthing and preparations by women prior to birthing periods. Provision of basic emergency comprehensive obstetric care (BEmOC) at the facility, user satisfaction, obstetric and abortion outcomes and knowledge of the health care operatives on standard emergency obstetric care (EmOC) were other reported outcomes. Obstetric maternal outcomes were assessed on maternal complications and live births, as well as on supervised and non-supervised deliveries. Economic/geographical outcome measures included cost associated with access to services, use of services, delays in seeking care, delays in reaching a health facility, delays in receiving appropriate care and place of birth. Patient-reported measures in studies on ANC assessed women's satisfaction with service use, service quality, decision making for services used and place of service utilization. Regarding contraceptive services, unmet need for contraception was measured based on current and ever use of contraception, contraceptive prevalence rates, intention for future use and women's knowledge of contraception use and benefits. Patient-reported measures related to obstetric care assessed unsafe abortions occurrence, reasons for seeking abortion, post abortion care, complication readiness, emergency planning steps, and awareness of danger signs prior to birth. 


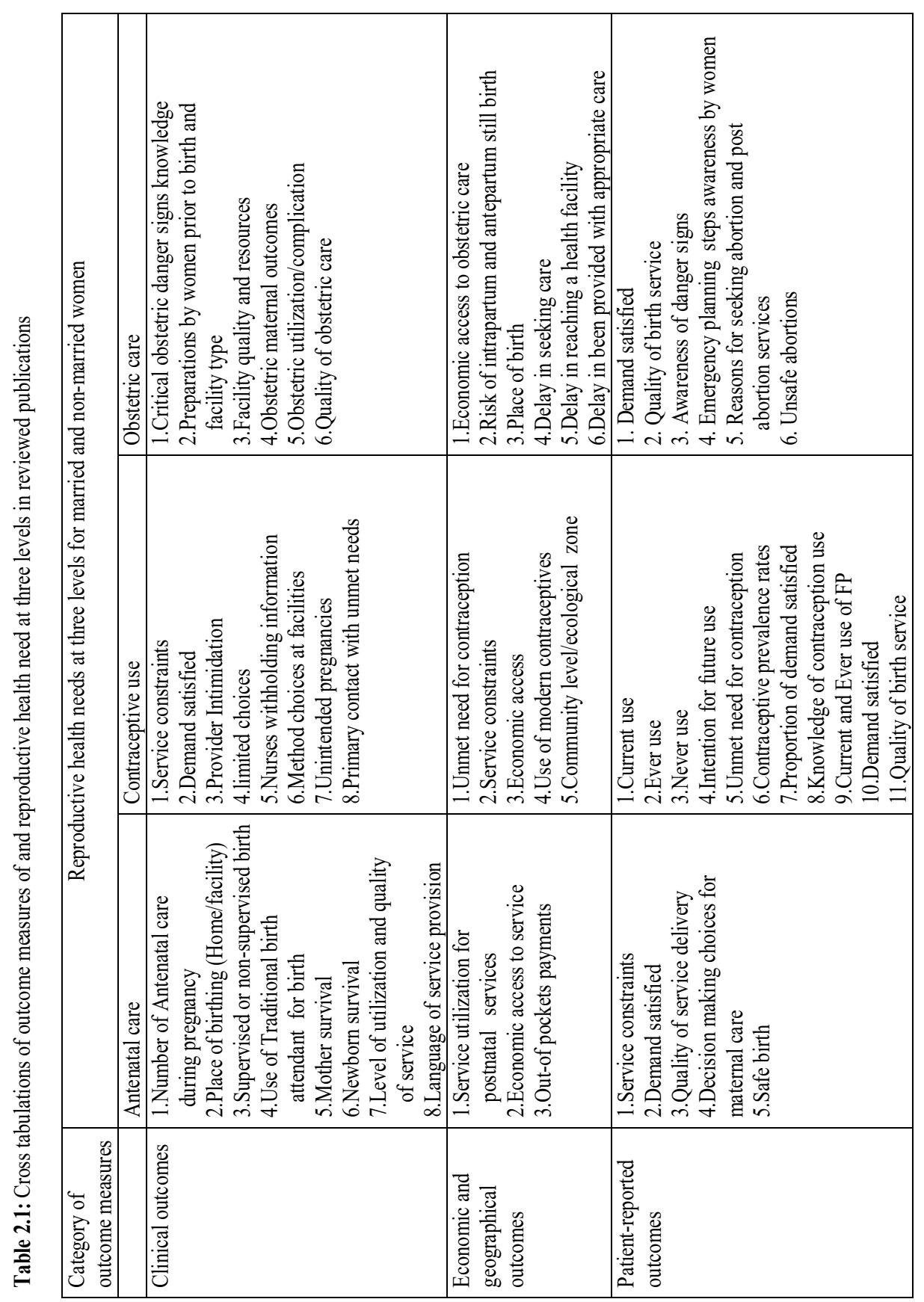


2.3.3 The extent of unmet need outcomes reviewed in publications

Table 2.2 summarizes three critical outcome measures reported in the publications reviewed. These outcome measures were mostly assessed and reflect only contraceptive use across clinical, economic and patient levels. Antenatal and obstetric care were not extensively highlighted in the reviewed publications and thus not included here. As evidenced in Table 2.2, unmet need for contraceptive use remains high across all countries. Ghana has the highest level of this type of unmet need across West Africa and Nigeria has the lowest rate. Contraceptive prevalence rates are highest in Ghana and lowest in Benin. Modern contraceptive prevalence rates reflect current acceptance and use of modern methods of contraceptive for all age groups. Hence, low rates depict low use and acceptance of modern contraceptive use may translate to low use in the future. Adolescent's birth rates, though declining, still remain high and stagnant among adolescent girls in West Africa (see Table 2.2). 


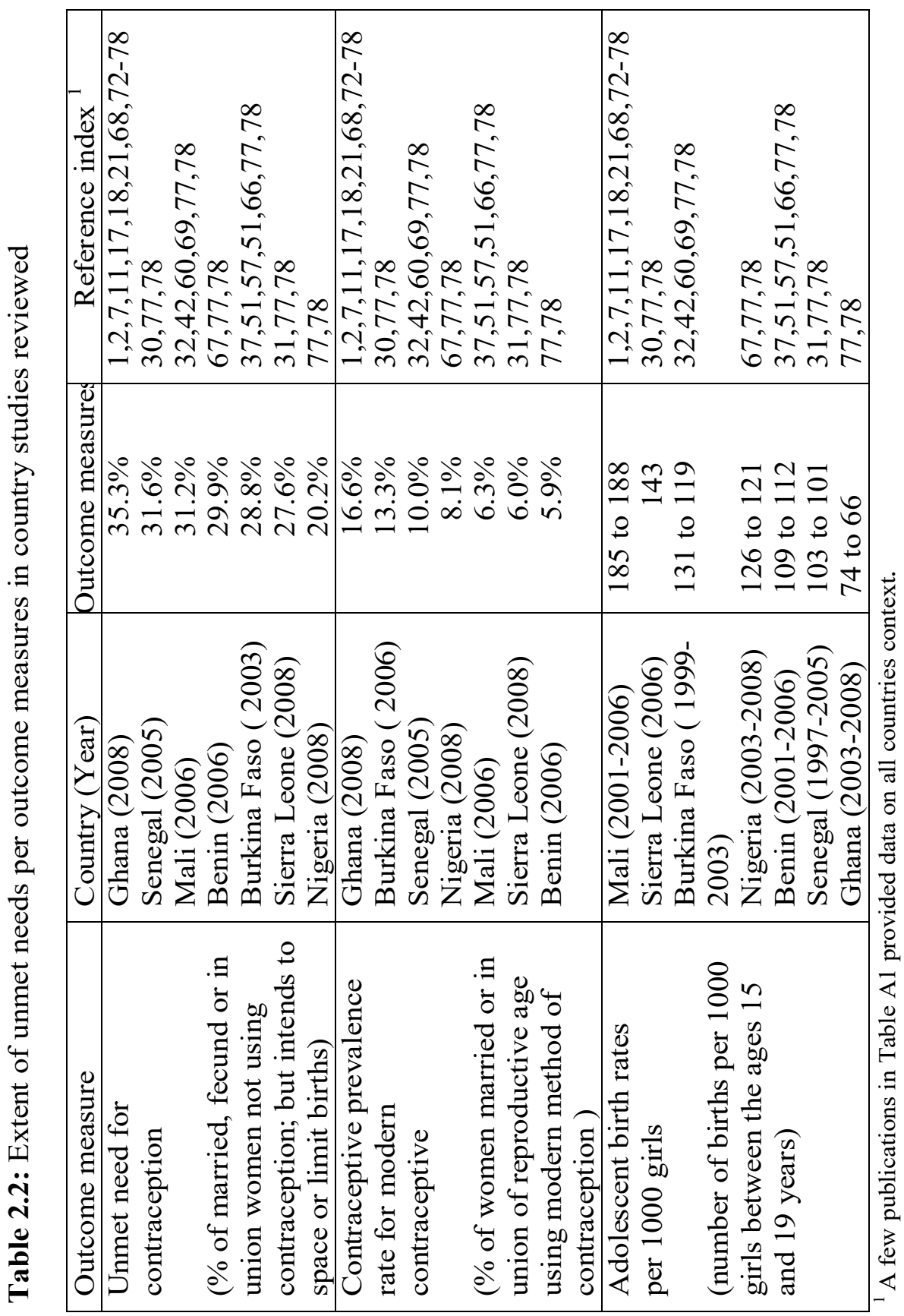




\subsubsection{Specificity on determinants in reviewed publications}

The main determinants reviewed in the publications are summarized in Table 2.3. Socio- cultural determinants were influenced by the perceptions of what constitute marriage and family, thus creating unequal power and gender disparities, affecting mainly women in attaining their needs for reproductive health.

Additionally, socio-cultural factors influencing antenatal, contraceptive, and obstetric care utilization were observed to be driven by the desire for a large family size by male spouses, community and individual perspectives of what is socially acceptable and worthwhile for women as well as some religious doctrines surrounding womanhood. Others such as women feeling disempowered owning to their poor quality of life and poor spousal communication, women's desire to secure their marriages and attract their husband's love and attention were also observed to stem from socio-cultural beliefs and perspectives.

Socio-economic measures such as the socio-economic wealth of a woman or household wealth were found to be key determinants in most publications for access and service use. Even in environments where women owned economic assets and resources, their economic value is seen as reduced since the male spouse socio-culturally is seen to own and control household resources. Socioeconomic factor such as money was seen to impact travel cost, cost of service provision and catastrophic expenditures on household wealth and income.

Poor knowledge concerning lifesaving skills (LSS) by health staff in delivering quality and efficient services was reported in some studies. Low or poor educational status of women and their knowledge of danger signs, knowledge of where to access services and the risk associated with service were also key determinants influencing service utilization in some settings. Conspicuously missing in almost all reviewed publications was the role health provider's play in influencing the services use through their educational sessions for women at the clinic or community setting as outlined in other studies (Stephenson, Baschieri, Clements, Hennink, \& Madise, 2007). Overall, single determinants alone were never observed in publications. Socio-demographic characteristics and institutional factors were the main driving indicators of current and future determinants for service utilization. Thus, the determinants reviewed indicate that, several factors either tend to work towards greater efficient use or deny access to millions of women from attaining their needs to reproductive health. 


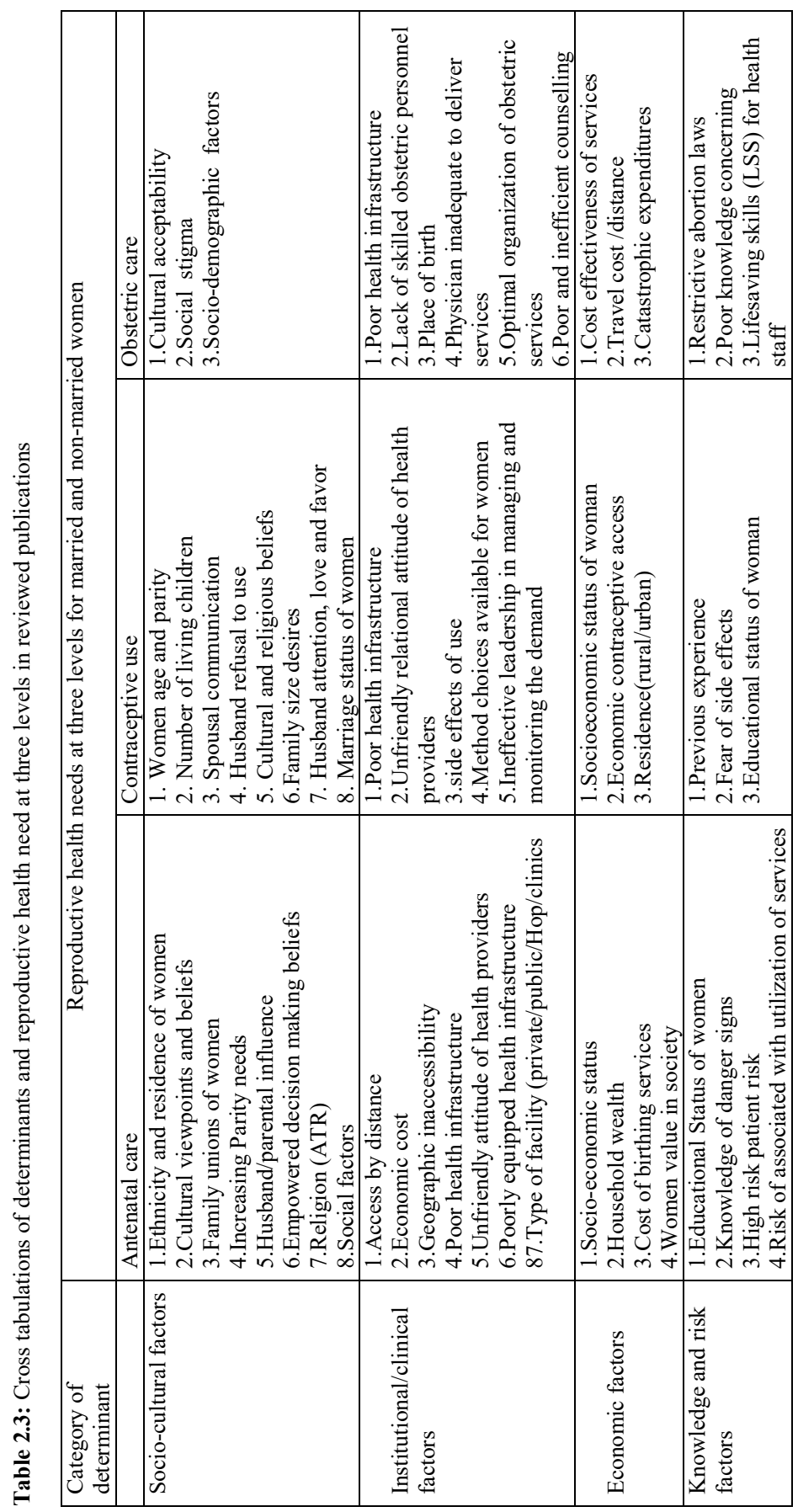




\subsubsection{Unmet needs and gender constraints identified in publications}

Review results show male dominance and authority on reproductive health needs among women in West Africa, which have a strong impact on women's reproductive health needs (Adongo et al., 1997; Chigbu, Onyebuchi, Onwudiwe, \& Iwuji, 2013; C. Izugbara et al., 2010; Ogu et al., 2012; Olayemi, Bello, Aimakhu, Obajimi, \& Adekunle, 2009; Olugbenga-Bello, Abodunrin, \& Adeomi, 2011; Omeje, Oshi, \& Oshi, 2011; Somé, Sombié, \& Meda, 2013). As mentioned above, this is driven by socio-cultural customs and beliefs of what constitute marriage, family and reproduction in these settings. These attributes were observed to have created power inequalities and gender disparities, affecting mainly women in attaining their needs for reproductive health (Halley Crissman et al., 2012; Eliason et al., 2013; Kodzi, Johnson, \& Casterline, 2012; Omeje et al., 2011; White, Dynes, Rubardt, Sissoko, \& Stephenson, 2013).

The results also point to the huge infrastructure deficits and ill equipped birthing conditions in poor resource settings as a major constraint for women to meet their reproductive health needs (Adanu, 2014; Dogba et al., 2011; Erim, Resch, \& Goldie, 2012; Okoli et al., 2012; Oyerinde et al., 2013; Payne et al., 2013). Community effects in relation to geography, cultural and religious beliefs are other major obstacles for women's attainment of their reproductive needs (Nikiema, Haddad, \& Potvin, 2012; Olugbenga-Bello et al., 2011). Certain environments tend to "devalue" women and reinforce strong power relations among men and women. Restrictive abortion laws in some countries also make women resort to adopting clandestine unsafe abortion strategies, which possess greater risk for women to survive later pregnancy and child bearing (Hill, Tawiah-Agyemang, \& Kirkwood, 2009; Payne et al., 2013).

\subsection{Discussion}

\subsubsection{Extent of unmet needs among women in West Africa}

The results of this review indicate a high level of unmet reproductive care needs reported by women in West Africa. Overall, contraceptive unmet need levels are a critical outcome measure for women reproductive health across the region. However, the more specific outcome measure of modern methods of contraceptive use is also important if the measure is to remain relevant and precise in capturing gaps for improving contraceptive supplies. We observed in the review that these two indicators can be contradictory. For example, Ghana shows the highest rate for unmet contraceptive needs but also a highest modern contraceptive prevalence rate, compared to the rest of countries in the review. Thus, Ghana has 
succeeded in increasing women's awareness of the need of contraceptives but has not achieved universal access to contraceptive services. Similarly, Benin has a high level of unmet contraceptive need but also a low prevalence rate of modern contraceptive. Thus, efforts to bridge the wide disparities are necessary to make visible progress on meeting women's health needs. This applies to the rest of the countries as well. Although the use of modern contraceptive is higher in countries such as Burkina Faso and Ghana, rates found in this review are still rather low compared to the rest of the developed world. Further steps need to be taken to improve the reproductive health delivery systems in West Africa.

Indices across West Africa on adolescent birth rates, contraceptive prevalence rates, and unmet need levels reflect poor and lagging policies of fertility, poor accountability in the health sector and the poor socio-economic growth across the region. Although various policies exist across West Africa aimed at improving reproductive health outcomes (as outlined in the introduction section), they are not well implemented and do not assure comprehensive coverage. There is no substantial progress in assuring free and adequate maternity care services. A few advantaged women across these countries may be contributing to national and country context progress on the critical indicators reviewed in this chapter. Thus, current reproductive measures for evaluating reproductive health needs appear broad and do not consider country differences in socio-economic development, political stability, and the health system across countries reviewed. There is a need for context specific outcome measures and determinants for tracking progress in each country. In particular, data are not available for all countries for the same year, and also within a country for several years. There is a need for the development of reliable data by strengthening the health surveillance and information management systems across the health systems in the region.

\subsubsection{Key determinants to women reproductive health in publications}

The reviewed publications indicate that social and cultural barriers to the use of reproductive health services are present in all countries although the degree and extent varies by country. Economic access was reported to be a major contributory factor for the use of services by women, depending upon the socioeconomic status of women seeking care, household wealth status, and the cost of service provision at the point of use. Even when out-of-pocket payments are not an ultimate determinant of utilization; distances that women have to travel were seen to influence health outcomes such as place of birth, demand satisfied, and maternal death. The geographic distributions of health staff as a measure further compounds access issues as evidenced in this review. Efforts at bridging access 
by various governments must start with providing the enabling infrastructure to bridge institutional determinants for intended users.

The effects of women's educational status and knowledge of risk factors was associated with decisions to adopt or not to adopt services in most reviewed publications for countries such as Nigeria, Ghana, Mali and Sierra Leone. Even in settings where women viewed knowledge as a non-important determinant to meeting their reproductive needs, their inability to read and recollect properly next visit days for services impeded on effective service provision by health providers. Quality and satisfaction of the place of service utilization and the presence of a skilled attendant during service use were observed as determinants and measures used coherently in almost all countries reviewed in this study. These outcomes related to quality and satisfaction, have the tendency to determine the use of care. Improving quality and satisfaction reassures and addresses new users concerns and has the potential to improve women future reproductive services use.

Additionally, our findings also highlight the role of the male spouse in influencing women reproductive behaviors. Poor spousal communication shows the lack of an equal "playing field" for women at the family level in terms of decision making. Traditional family settings in West Africa view the family as a single unit governed by the single head which is the man. Opportunity for dialogue is scarcely available concerning the daily management issues at the family level prescribing men with the social responsibility as breadwinners. This was most evident in strict religious dominated environments and countries such as Nigeria, Benin and Niger whose chapters were reviewed. In families where women face opposition and obstacles to reproductive service utilization, covert use is sometimes adopted. However, covert use is no option for women using services in settings were privacy is not assured since it can warrant marital problems.

Although our literature search was systematic and assessed all related studies within the desired scope, it is possible that we missed relevant publications, e.g. publications reported in non-English journals and most recent studies not yet reported. Also, eligible studies were only found for 7 out of the 15 West African countries. No study was found for Guinea Bissau (at least among the English publications). Two countries, Nigeria and Ghana had a relatively high number of studies included in the review compared to the rest of the countries. Despite this limitation on the country coverage and number of publications finally reviewed, the findings of this study remain relevant for the selected West African countries because of the similarities in the social, community and regional context, as well as health systems across the region (Alkema, Kantorova, Menozzi, \& Biddlecom, 2013; Withers et al., 2013). In view of this, our conclusions are essential for the 
improvement of reproductive outcomes in the entire region, including countries where such studies are absent. In addition, the absence or low proportion of studies in some West African countries on the inclusion criteria, as indicated by our review, confirm the need of more policy and research attention to women's reproductive health needs in the region.

\subsection{Conclusion}

This chapter focused on analyzing outcome measures and determinants of unmet reproductive care needs in West Africa based on a systematic review of previous studies. The findings from this review show the existence of a broad range of outcome measures and determinants for evaluating reproductive care needs applied across the countries. Evidence shows a high rate of unmet reproductive care needs despite the progress in some countries. This is associated with poor socio-economic indices, deep and varied social norms and beliefs across cultures and traditions that place marriage and a women's reproductive health needs as the male spouse responsibility at the family unit level. Critical too are the poor institutional arrangements, rising adolescent birth rates, poor economic status and poor knowledge, which further divest women reproductive health seeking behaviors in these societies. The multiple social, economic and environmental factors that affect women meeting their reproductive health needs along the continuum of care, require the involvement of user, provider and policy-makers to bring the desired changes in the countries. In pursuing these ambitions, reforms that improve access (e.g. legislative reforms on restrictive abortion laws) will go a long way to improve unmet need for obstetric care in West Africa. Although outcome measures and determinants identified in the review remain critical progress benchmarks globally, they are rather broad. There is need to incorporate country level context measures in benchmarking progress on reproductive health needs among women to better evaluate each countries' progress on reproductive health. 



\section{CHAPTER 3 \\ Focused maternity care in Ghana: results of a cluster analysis}

\section{Draws upon published work:}

Ayanore, MA., Pavlova, M., \& Groot, W. (2016). Focused maternity care in Ghana: results of a cluster analysis. BMC Health Services Research, 16:395. DOI: 10.1186/ s12913-016-1654-5 


\section{Abstract}

Introduction: Ghana missed out in attaining MDG 5 in 2015. The provision of adequate prenatal and postnatal care remains problematic, with poor evidence on women's views on met and unmet maternity care needs across all regions in Ghana. This study examines maternal care utilization in Ghana by applying WHO indicators for focused maternal care utilization.

Methods: Two-step cluster analysis segregated women into groups based on the components of the maternity care used. Using cluster membership variables as dependent variables, we applied multinomial and binary regression to examine associations of care use with individual, household and regional characteristics.

Results: We identified three patterns of care use: adequate, less and least adequate care. The presence of a female and skilled provider is an indicator of adequate care. The lack of female autonomy, widowed/divorced women, age and parity were associated with less adequate care. Care patterns were distinctively associated with the quality of health care support (skilled and female attendant) instead of with the number of visits made to the facility. Across regions and within rural settings, disparities exist, often compounded by supply-related factors.

Conclusions: Efforts to address skilled workforce shortages, greater accountability for quality and equity, improving women motivation for care seeking and active participation are important for maternity care in Ghana. 


\subsection{Introduction}

Ghana introduced a fee-exemption policy for maternity care in 2007 to accelerate the progress towards the achievement of MDGs 4 and 5. This was also aimed at improving maternity care utilization. This and other health policies are closely tied to the first population policy of 1969 (revised in 1994) and rooted in the conviction of providing conditions for safe motherhood in Ghana (see Chapter 1). The country launched a new health policy 2007 dubbed "creating wealth through health" with a vision to attain a middle-income status by 2015 $(\mathrm{MOH}, 2007)$. With a renewed focus on improving human capital and achieving the MDGs, targeted interventions to scale up high impact and rapid delivery health interventions were stepped up. Efforts at improving health surveillance and accountability to ensure improved health access and quality, received multisectorial recognition $(\mathrm{MOH}, 2007)$. These interventions and the introduction of the NHIS provided a new pathway for funding healthcare, improving healthcare access whiles shouldering the depleting effects caused by "cash and carry system" in the early 1990s (see Chapter 1).

Despite these gains, the number of childbirths assisted by a skilled attendant (physician, midwife/nurse or community health officer) in 2008 was low (59\% of all deliveries) compared with the level of ANC utilization (95\% of pregnant women) (GSS, GHS, \& ICF, 2009). Recent evidence indicates that an estimated $74 \%$ of national deliveries are attended by a skilled provider (GSS et al., 2014). Additionally, maternal and infant mortality rates (380 per 100,000 live births and 52 per 1,000 live births respectively) remain at unacceptable levels. Despite maternal and child friendly center campaigns, and the expansion of Communitybased Health Planning and Services (CHPS), many births remain unattended by skilled professional care. Many broader social determinants of health care are found to have a great impact on women maternity care utilization in poor settings (Arthur, 2012; Gayawan, 2013).

Previous studies in Ghana on maternity care investigated the low care quality and travel distance, skilled provider distributions, out-of-pocket payments for maternity care when insurance is absent, facility type (public/private) and delivery status (see Chapter 1). Empirical evidence also enumerates patient inequalities, provider type relations, socio-economic determinants, visits and utilization effects on neonatal deaths (Addai, 1998; Asamoah et al., 2014; AtunahJay et al., 2013; Lambon-Quayefio \& Owoo, 2014). These studies however, analyze users of maternal care as a single group and provide trends over a specific period. None of these studies provides a segmentation of women's met and unmet care needs based on care components received according to WHO guidelines for focused maternity care (see Chapter 1). Such segmentation is important for 
understanding the standards of care met and unmet. This is critical for examining the maternity care delivery packages for improved utilization in settings where skilled professional care is low.

This chapter examines the components of antenatal and postnatal care utilization in Ghana at three levels; pre and postnatal care, facility utilization and medication care at last birth. By using data from the 2008 Ghana Demographic Health Survey (GDHS) dataset, and employing a cluster and regression analysis, the study offers evidence on the adequacy of care received (adequate, less adequate or least adequate) by different groups, and associated reasons for these variations. The study provides a "window of opportunity" to examine the critical salient factors affecting women needs when seeking maternity care. This will inform policy decisions at all levels; district, regional and national for improved maternity care at the primary level for women in Ghana.

\subsection{Focused maternity care in Ghana}

Current interventions in Ghana for safe motherhood aim to improve emergency obstetric care, family planning, and surveillance systems for maternal mortality monitoring and reporting. Other interventions involve the use of novel techniques (High impact Rapid Delivery) to scale up the most targeted interventions $(\mathrm{MOH}, 2008)$. Notwithstanding this, within urban and rural areas, maternity care cannot be dissociated from the broader challenges of the general health care system (see Chapter 1). In a baseline study in 2005, it was shown that access and utilization were inadequate for both basic and emergency obstetric care $(\mathrm{MOH}, 2008)$. Inequality in access to skilled birthing exists. An estimated $36 \%$ of women have access to public facilities while $9 \%$ has access to private facilities. Rural, educational and geographical variations account for this. Earlier national policy directions recognized the role of Traditional Birth Attendants (TBAs), but the recent policy focus has centered on three types of professionals: physicians, midwives/nurse and community health staff. Current data (GDHS, 2008) suggest that only $10 \%$ of women are attended by a physician and $41 \%$ are attended by midwife/nurse during birth. A significant policy progress was the introduction of a fee-exemption policy and its subsequent take over under the national health insurance scheme to remove financial barriers for care (see Chapter 1).

The assurance of all essential components of maternity care is vital to guarantee a continuum of care for women during and beyond pregnancy (see Box 3.1). 


\begin{abstract}
Box 3.1: Components of WHO "focused care" and Ghana success maternity interventions

In Ghana, maternity care developments are grounded in the WHO guidelines for a "focused ANC" for all expectant mothers, including: identifying and managing all pregnancy complications, identifying and treating all concurrent illness whiles taking steps to avoid the complication exacerbation through prevention and case management of anemia and intermittent treatment for malaria (IPTp). Others such as micronutrient supplementation, two doses of tetanus immunizations, education and sensitization on HIV/AIDS, healthy lifestyle behaviors and the monitoring of very vital signs during every stage of pregnancy, are provided. Assisted birth by a professional at a facility that has the capacity to provide the needed care for a good pregnancy outcome is also essential. Adequate postnatal care is characterized by the presence of skilled care during and for the first 42 days after birth. Appropriate postnatal check up on the mother and newborn critically depends on receipt of post check after delivery, timing of care received (at least within 1 week for hard to reach areas) and the provider type (skilled or unskilled) providing care. Although ANC alone cannot significantly impact maternity outcomes, its adequate provision provides an entry point for integrated care, contributes to skilled utilization at birth and links community care structures with women. Maternity care is delivered in Ghana in both private and public facilities, encompassing all categories of maternity homes, clinics, CHPS compounds, health centers and hospitals. Most maternity homes, clinics (all levels), health centers and regional hospitals exist in urban areas while CHPS centers, health centers or district hospitals if they do exist, provide care for those living in rural areas. Evidence based approaches to improve birthing outcomes exist in Ghana, such as the community participatory approach (Zorko initiative), Amansie west, Koforidua, and Tamale teaching hospitals experiences. Understanding continuously women in changing demands and needs influencing critical care components for maternity is important to make current strategies and interventions relevant.
\end{abstract}

Source: Extracts from synthesis report of the MOH, 2008.

\title{
3.3 Methods
}

The 2008 GDHS dataset was used in this study. The GDHS is a nationally representative survey that applies standardized data collection instruments to collect information to inform health policy planning and implementation across all 10 regions in Ghana. The 2008 GDHS was implemented by the Ghana Statistical Service and Ghana Health Service with technical support from ICF Macro and MEASURE DHS program (GSS et al., 2009). Specifically, a two stage-point cluster and systematic sampling was applied. The first stage sampling was made up of 412 clusters selected from an updated master sampling frame constructed from the 
2000 Ghana population and housing census. A listing operation was conducted between June and July 2008 in all selected clusters to provide the sampling frame for the selection of households in the second stage of clustering. Table 3.1 provides an overview of the household's distribution and enumeration areas per region.

Table 3.1: Households distribution and enumeration areas in the 2008 GDHS

\begin{tabular}{|l|l|l|l|}
\hline Region & $\begin{array}{l}\text { Percent distribution } \\
\text { households 2000 census }\end{array}$ & $\begin{array}{l}\text { Percent distribution } \\
\text { households 2008 GDHS }\end{array}$ & $\begin{array}{l}\text { Total number of } \\
\text { Enumeration areas } \\
\text { (EAs) }\end{array}$ \\
\hline Upper East & 3.9 & 6.8 & 28 \\
\hline Upper West & 2.2 & 7.3 & 30 \\
\hline Northern Region & 6.6 & 9.2 & 38 \\
\hline $\begin{array}{l}\text { Brong Ahafo } \\
\text { Region }\end{array}$ & 9.3 & 9.2 & 38 \\
\hline Ashanti Region & 18.4 & 16.3 & 67 \\
\hline Eastern Region & 12.3 & 10.4 & 43 \\
\hline Volta Region & 9.3 & 8.5 & 35 \\
\hline $\begin{array}{l}\text { Greater Accra } \\
\text { Region }\end{array}$ & 16.9 & 14.6 & 60 \\
\hline Central Region & 9.9 & 8.2 & 34 \\
\hline Western Region & 11.1 & 9.5 & 39 \\
\hline Total & 100 & 100 & 412 \\
\hline
\end{tabular}

Source: Data reported in the 2008 GDHS report

The second stage sampling applied a stratified, two-stage cluster design. A systematic sampling approach selected the households listed in each cluster. The initial objective was to have 30 households per cluster. However, a weighting adjustment procedure was applied according to the population distribution in the 2000 census. Also, one cluster was excluded at the time of data collection due to security reasons. This provided a final sample of 12,323 households nationwide (GSS et al., 2009). A total of 11,778 occupied households were interviewed during the 2008 GDHS with a response rate of $96.5 \%$. The sample selection procedure and the response rate enable separate estimates for respondents in all regions, as well as for rural and urban areas.

Women aged 15+ years were eligible to be interviewed if they were usual residents or visitors present in the household the night before the interview. In total, 4,916 eligible women were interviewed in the survey. This was made up of 2,162 urban and 2,754 rural eligible women. From them, 2,147 women (763 urban and 1,384 rural women) within reproductive age (aged 15-49 years) were included in our analysis because they reported childbirths within the last 5 years. All eligible women participants provided informed consent for the study. There 
was no deception of respondents and no attempts were made to cajole respondents into agreeing to something to which they may not have otherwise agreed. All information that may compromise anonymity of respondents was not retained on record (Adetunji \& Shelton, 2011). Approval for use of dataset was obtained from ICF Macro and MEASURE DHS.

\subsubsection{Dataset variables assessed}

The content of the questionnaire in the 2008 GDHS study was based on model questionnaires developed by MEASURE DHS and the 2003 GDHS questionnaire. Field pre-testing was carried out between June and July 2008. The questionnaire was translated from English into three main working languages: Akan, Ewi and Ga. The main data collection was carried out between September and November 2008 involving face-to-face interviews. From the detailed individual women questionnaire, three groups of relevant response variables related to pregnancy and postnatal care based on the WHO indicators as exemplified in Box 3.1 were assessed;

-Group 1 containing questions on prenatal care during pregnancy, including 15 questions on prenatal care use, attendant availability (skilled/unskilled) during antenatal visits, pregnancy complication awareness, knowledge and awareness of HIV/AIDS during pregnancy.

-Group 2 containing questions on facility utilization during pregnancy and after birth, including 6 questions to assess facility utilization during pregnancy and birth regarding frequency and place of utilization, as well as health checks before and after discharge.

-Group 3 containing questions on prenatal and postnatal pregnancy medications received, including 10 questions centered on prenatal and postnatal pregnancy medications received as part of prenatal and postnatal care.

The women in our sample were asked to provide the above information for their last childbirth during the last 5 years.

\subsubsection{Data analysis}

Cluster analysis, a multivariate statistical technique for grouping cases of data, was applied in our analysis. The purpose was to categorize women's responses into groups or clusters based on the patterns of similarities and dissimilarities of material care received during the last birth. The large sample size coupled with the mixture of categorical and continuous data required the application of a two-step cluster approach instead of other clustering approaches, such as hierarchical and 
k-means cluster analysis (Verma, 2013). The two-step clustering procedure allows for an exploratory identification of natural cases/objects within the large dataset. Differences among variables are determined by the loglikelihood distance measure.

The cluster analysis was carried out per group of questions (see data collection above) and for all questions together, resulting in 4 separate clustering procedures (software package SPSS 22). To avoid the exclusion of respondents due to a high rate of missing values $(>10 \%)$ in few variables, a category "no response" was created for these variables. Related to reliability and validity, the cluster analysis was repeated several times checking whether the cluster range remained fair to good. The clustering stage was ended when stable cluster membership groups were obtained. All clusters were generated automatically (no manual pre-selection of the number of the clusters) and produced fair to good cluster ranges. The 4 clustering procedures mentioned above, produced 4 cluster membership variables (see Figure 3.2-3.5). These membership variables were used as dependent variables in a subsequent binary/multinomial logistic regression analysis depending on the number of the clusters. For all regression models, the explanatory variables included the same set of individual and household socio-demographic characteristics, as well as regional characteristics. The significance threshold was $\mathrm{p}<.05$.

\subsection{Results}

In total, 2147 eligible women respondents who reported childbirths 5 years prior to the study were included in our final analysis. Descriptive statistics of the response and socio-demographic variables of respondents are presented in Appendix A and B. The results of the cluster analyses (based on the response variables) are first presented in this section, followed by the results of the regression analyses using the cluster membership variables as dependent variables and the socio-demographic characteristics as explanatory variables (absolute value of correlation between explanatory variable $<0.5$ ).

\subsubsection{Cluster findings}

We first present the results of the two-step cluster procedure based on all response variables (see Appendix B). Three distinct clusters with varied care components were generated. The cluster group distribution among the three clusters is presented in Figure 3.1. Overall, cluster group 1 can be categorized as a group that received adequate care (more skilled attendants, more information about signs of pregnancy complications, and more medication) compared with cluster groups 2 and 3 (see Figure 3.2). 


\section{Cluster analysis:all response variables}

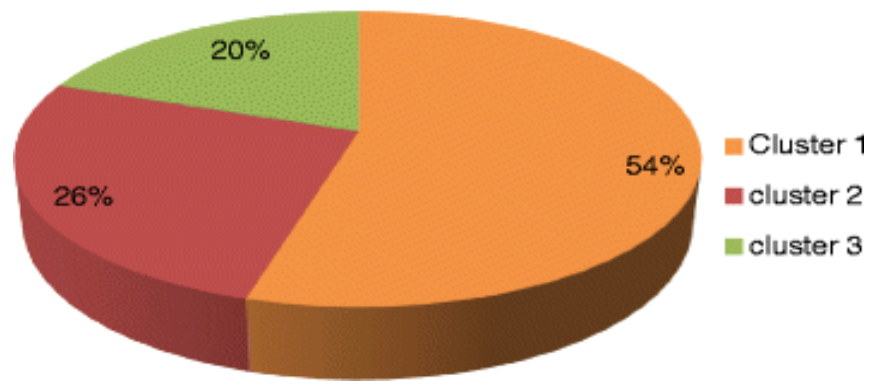

Fig. 3.1: Overall distribution of respondents in the cluster analysis. Note: Cluster 1 (adequate care), Cluster 2 (less adequate care) and Cluster 3 (least adequate worse care)

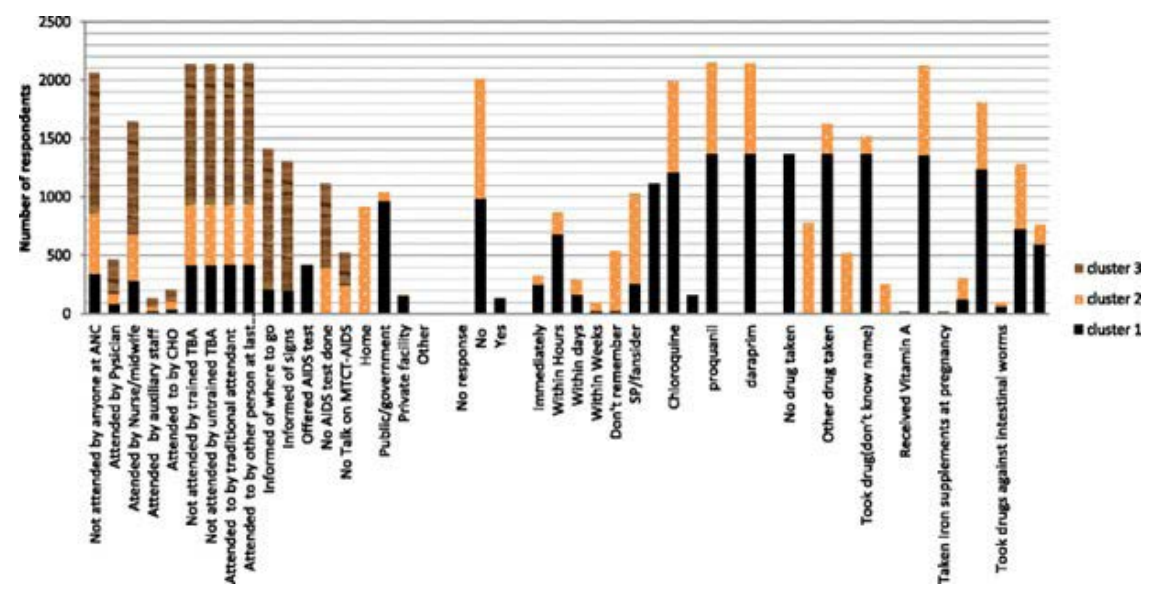

Fig. 3.2: Overall levels of care components received by women (15-49 years). Note: Cluster 1 (adequate care), Cluster 2 (less adequate care) and Cluster 3 (least adequate care)

From Figure 3.1 and 3.2, we see that cluster group 2 received less adequate care compared to cluster group 1 but overall better care compared to cluster group 3. Cluster group 3 received the least adequate care compared to the other two cluster groups. Details about the cluster patterns and quality are found in Appendix C. Specifically, compared to cluster groups 2 and 3, women in cluster group 1 were more likely to be attended by a physician, nurse/midwife and auxiliary staff, to have received more information about pregnancy signs and complications, received mother-to-child transmission (MTCT) of AIDS talks, offered AIDS test at ANC, tested for AIDS, delivered at public/government facility, delivered by caesarean, received quick health check-ups after birth, and received birth 
checks before discharge. Other distinctive features of cluster 1 included: more drugs against malaria and intestinal parasites, and postnatal vitamin A and iron supplements during prenatal and postnatal periods. Drugs against malaria for group 1 respondents were more likely to be SP/malafan/fansider, chloroquine and proquanil. Absence of any attendance was less likely in cluster group 1 and early visits for their first antenatal check-up was more likely compared to cluster group 2. However, antenatal visits for cluster group 1 were less likely than for cluster groups 2 and 3.

Women in cluster group 2 were most likely to be attended by a TBA. Compared to cluster groups 3, this group was more likely to have been attended by someone at ANC and was more informed about where to go with pregnancy complications, although these rates were lower than in cluster group 1. Women in cluster group 2 were also more likely to be informed on signs of pregnancy complications, offered an AIDS test and tested for AIDS, and provided talks on MTCT of AIDS compared with group 3, but less likely compared with group 1. Difficulties to recall the timing of the first postnatal check and birth in other place (than those specifically listed) were more common among cluster group 2 respondents compared to cluster group 1 and 3 . Women who took no drugs for malaria or could not recall what drug were taken, were also more likely to belong to cluster group 2 compared to cluster group 3, but less compared to cluster group 1. The majority of women in cluster group $2(70.0 \%)$ did not take drugs against any intestinal parasites, which was a higher non-use than in group 1 and 3.

Postnatal vitamin A and iron supplement intake was more likely for cluster group 2 respondents compared to cluster group 3 . Both cluster 2 and 3 reported less vitamin A intake compared to cluster group 1. Antenatal visits for cluster group 2 was however more likely than for cluster group 1 and less compared to cluster group 3 . The early timing of women first antenatal visit was most likely in cluster group 1, less in cluster group 2 and least in cluster group 3. Cluster group 3 women were most likely to be attended to by a TBA (trained/untrained). A high share of this cluster group also reported not being attended by someone/ anyone, not being offered AIDS test nor tested for AIDS, and being less likely to have talks on MTCT of AIDS compared with groups 1 and 2. Home delivery was most likely in cluster group 3 compared with cluster groups 1 and 2. Postdelivery check-ups in cluster group 3 were reported to take place within one or more weeks on average. Health checks before discharge after delivery for cluster group 3, were comparable with cluster group 2 but less likely compared with cluster group 1. The use of SP/malafan/fansider and chloroquine as a malaria prevention was reported by cluster group 3, but less often compared to cluster group 1. Women in cluster group 3 were more likely not to take any drug against malaria or recall the name of malaria drugs taken during pregnancy. This was 
less reported among cluster group 2 and least among cluster group 1. Vitamin A intake and iron recipients for cluster group 3 were comparable with cluster group 2 , but less compared with cluster group 1. Cluster group 3 recorded most visits for antenatal check-ups compared with cluster groups 1 and 2, but this group was less likely to have attended earlier first antennal care compared with cluster groups 1 and 2. To confirm the results of the cluster analysis that included all response variables, we also present the results of three separate cluster analysis performed per group of questions using the two-step cluster approach (see Figure 3.3-3.5).

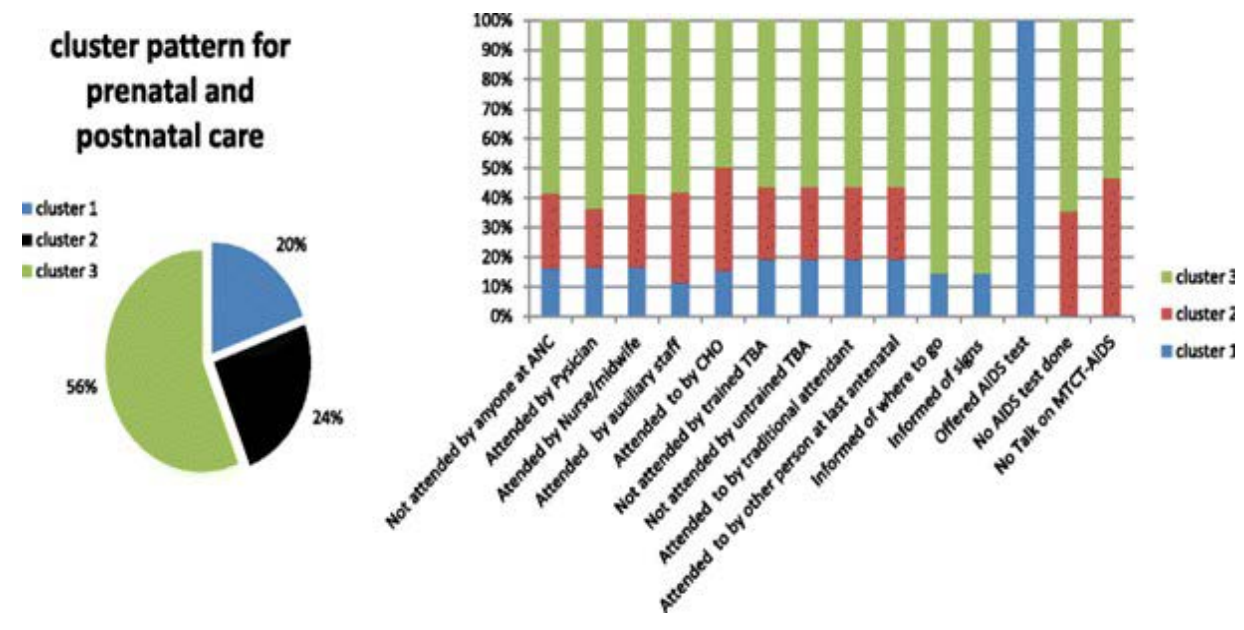

Fig. 3.3: Prenatal and postnatal care cluster characteristics for women (15-49 years). Note: Cluster 1 (adequate care), Cluster 2 (less adequate care) and Cluster 3 (least adequate care)

The results are comparable to those presented in the overall cluster analysis (Figure 3.1-3.2). Specifically, Figure 3.3, 3.4 and 3.5 show the proportion of women respondents clustered under each cluster category in the subsequent cluster analyses. 


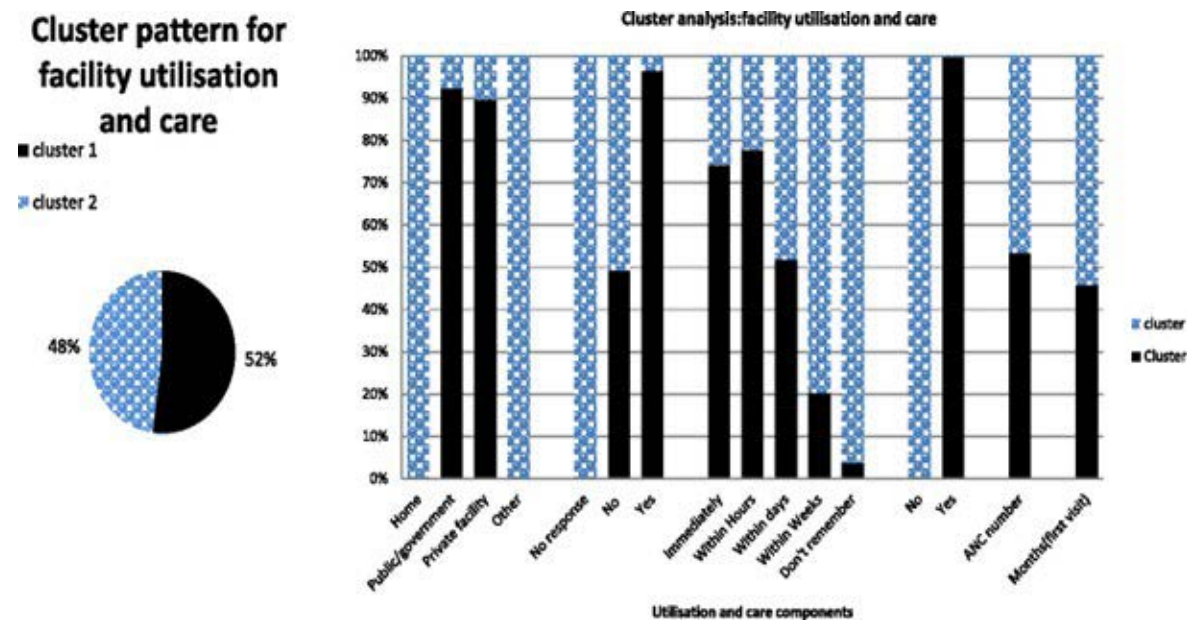

Fig. 3.4: Facility utilization and other care components received by women (15-49 years). Note: Cluster 1 (adequate utilization care), Cluster 2 (less adequate utilization care)
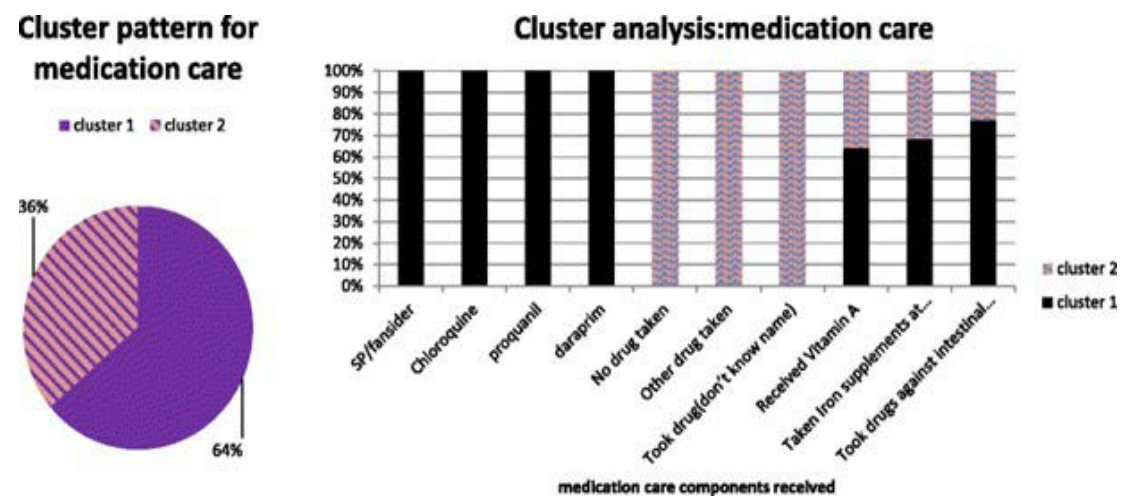

Fig. 3.5: Medication care components received by women respondents (15-49 years). Note: Cluster 1 (adequate medication care), Cluster 2 (less adequate medication care)

\subsubsection{Results of the multinomial and regression analysis}

Regression results are presented here to ascertain the associations between the adequacy of care received by women during pregnancy and childbirth and sociodemographic characteristics. We present the results of four regression analyses (multinomial and binary) where the cluster membership variables generated by the four cluster analyses are used as dependent variables respectively. An identical set of explanatory variables is used as independent variables in all four regressions 
(see Table 3.2). In the first regression model, where the cluster membership is based on all response variables, two geographical regions (Volta and Upper West) appeared to have received significantly less adequate care. Also, the likelihood of receiving less adequate care was more for women within the poorest wealth quartile (OR=1.754, C.I 0.999-3.078). The likelihood of receiving least adequate care was significantly associated with women without health insurance, and those who never slept under insecticide treated nets (ITNs). Additionally, women's age was also significantly associated with receiving the least adequate care (with older women more frequently receiving least care). The odds of receiving least care was 2.3 times higher in the absence of a female provider at the point of care provision $(\mathrm{p}<0.003,95 \%$ C.I 1.319-3.955) and 2.0 times higher for widowed/divorced women ( $<<0.005,95 \%$ C.I 1.244-3.371). However, concerns of no drugs available for treatment, difficulty with access (distance/transport), residence place (rural) were also significantly associated with least adequate care received.

The regression model based on membership variables generated by the three cluster analyses groups showed similar results. Close associations related to regional variations and wealth status were observed in these three regressions as well. Similar close associations were also found to relate to respondents' parity and autonomy (final say on health). Regarding prenatal and postnatal care and information received, parity and respondents' own say on their health needs had a positive association with receiving least adequate care. All other factors associated with the least adequate care (women without health insurance, and those who never slept under ITNs, no female provider, widowed/divorced and residence type) were similar to those reported above. The probability of receiving least adequate care was 1.7 times higher for rural compared to urban residents $(\mathrm{p}=0.002$ 95\% C.I 1.229-2.473). However, Volta, Upper West and Northern regions also showed close associations with less adequate care. The odds ratio for women living in the Northern region to receive less adequate care was twice higher compared with the other nine regions. Also, the influence of male partner on woman's health status was positively associated with women who reported less adequate care. 


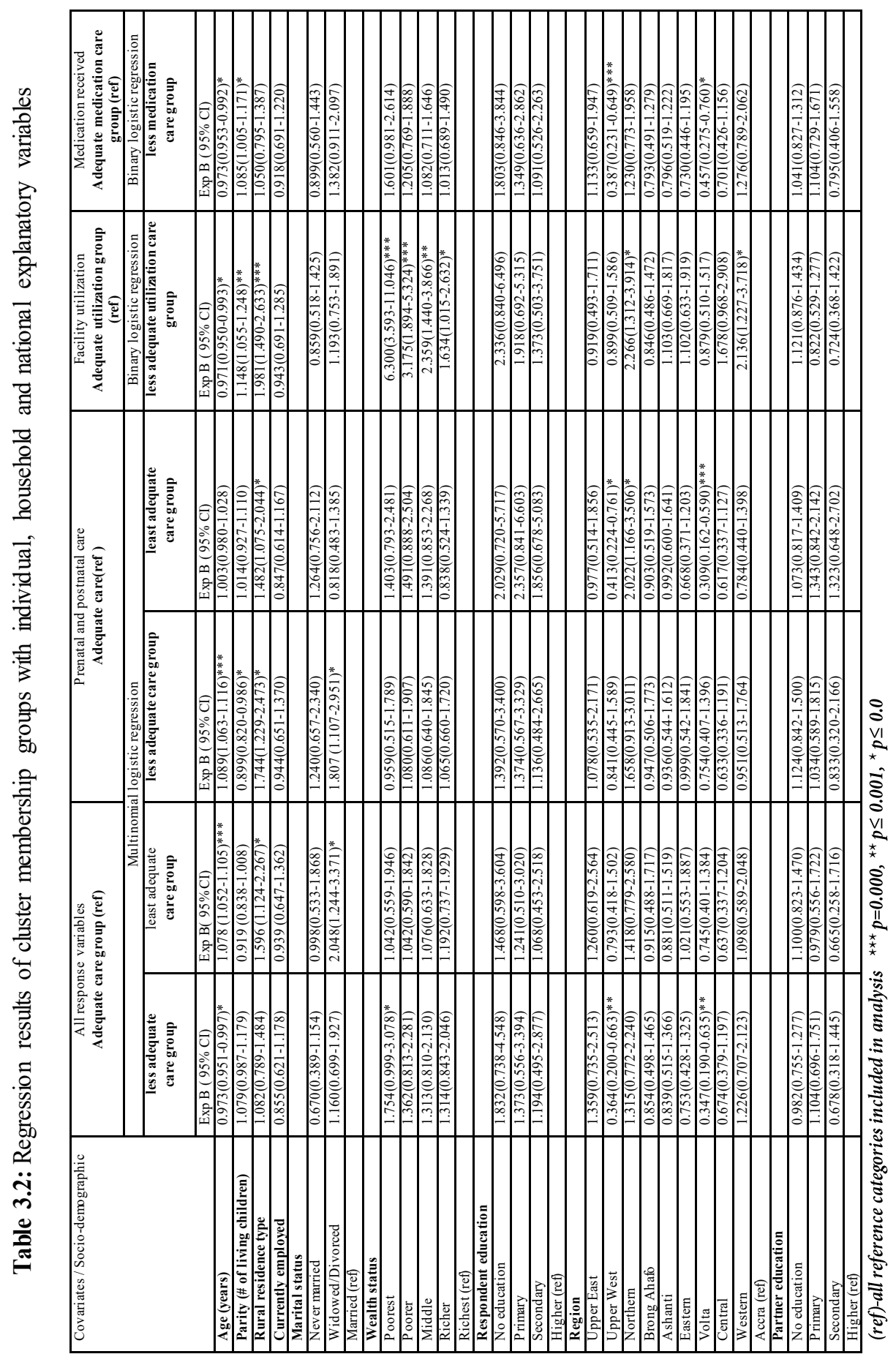




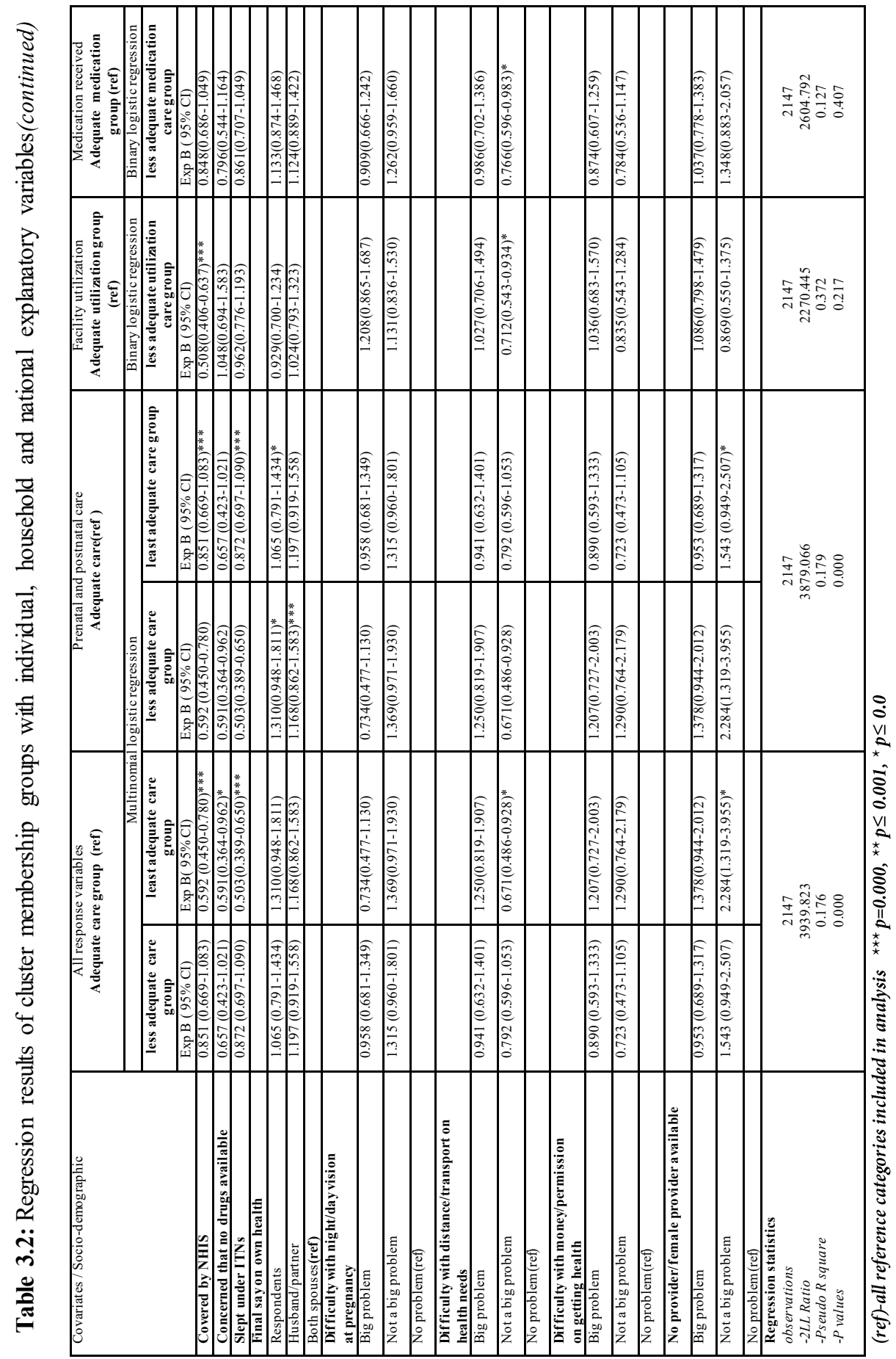


The odds of less adequate care was greater for women who expressed the absence of a female provider at the point of service use ( $\mathrm{OR}=2.28495 \%$ C.I 1.3193.955). Female autonomy (final say on women health) was positively related with less adequate care. A woman's place of residence had a positive association with less adequate care.

Wealth status (decreasing wealth) had a strong positive association with less facility utilization. Women within the poorest wealth quartile were 6 times more likely to utilize less care ( $\mathrm{p}=0.000,95 \%$ C.I 3.593-11.046). Women in the Northern and Western regions expressed low facility utilization levels. Rural women were most likely to report less utilization of facilities compared with urban residents. High parity levels and women without health insurance were associated with less utilization. Higher parity was positively associated with less facility utilization, less medication care as well as worse care observed for all individual cluster membership groups. Poor access factors (distance/transport) and two regions (Northern and Western) showed strong associations with lower utilization levels. Overall, age, parity, regional variations, residence type (rural/urban) and not covered by health insurance were observed as the most significant factors with a greater propensity to influence care in all membership groups. Respondents and their partner educational status had no significant associations with less adequate or least adequate care received. Other factors, such as employment status and women reported difficulties in getting money for care, showed no significance for the least care observations recounted among women.

\subsection{Discussion}

In this study, we examined maternal care utilization components in Ghana based on the 2008 GDHS dataset. Individual, household and regional factors were used as explanatory variables to ascertain possible differences between those who experienced adequate care and those who received less or least adequate care. More than half of women in our sample received adequate prenatal and postnatal care. Worrying is the equally approximate share of women who received less and least adequate care. Nevertheless, even among those who used adequate care, there were some drawbacks in the utilization of the recommended focus care components as overall antenatal utilization was low compared with less and least adequate care groups. This study specifically establishes that the frequency of antenatal visit is not associated with the adequacy of care received. Indirect cost and poor access, mediated through health insurance and travel cost, impact greatly on focused maternity care in Ghana. The results also show inequities across the regions with regard to the adequacy of care received, emphasizing that optimal maternal care at a national level was not attained in Ghana. 


\subsubsection{Overall prenatal and postnatal care experiences}

Our cluster analysis findings reinforced that women who were attended by skilled personnel were more likely to report adequate care irrespective of the frequency of antenatal visits. Adequate care was observed in cluster patterns related to skilled-provider presence and type (male/female), instead of the number of visits to the facility, reaffirming that the frequency of visits did not guarantee good care. Overall less adequate care was attributable to wealth and regional variations. Volta, Upper West and Northern regions had more women reporting less adequate care. The evidence from these regions for less adequate care shows regional variations in service utilization in Ghana. The Northern region was observed to have the least adequate care in Ghana. The Northern and Western regions showed lower levels of facility use and medication care. According to the 2008 GDHS report, the Northern region had the highest fertility rate of 6.8 in Ghana. Poor access and increasing parity as well as service quality and variations between rural and urban areas could have accounted for differences in these four regions (Volta, Upper West, Northern and Western). Although wealth status did not impact directly on the least adequate care received, its indirect impact elicited by the lack of public health insurance, was evident as a strong positive associate for least adequate care. The lack of health insurance contributing to least adequate care could have resulted in a decline in facility visits since direct out-ofpockets payments would create financial obstacles to seek care. This association is supported by evidence that women with public health insurance are likely to improve utilization of health care services (Baker, Sudano, Albert, Borawski, \& Dor, 2001; De Allegri et al., 2011; Do, Soelaeman, \& Hotchkiss, 2014; Okafor, Obi, \& Ugwu, 2010; Zere, Kirigia, Duale, \& Akazili, 2012).

The concern of no drugs and women's inability to use ITNs contributed to the least adequate care, providing corroborations with earlier findings in Ghana that health interventions such as sleeping under ITNs (child and pregnant woman) are not wealth manifested, although they remain significant to bridge inequities for maternal and child health outcomes (Zere et al., 2012). Women's recount of drugs unavailability resulting in least adequate care is supported by previous maternity care findings that supply driven factors regulated by weak health delivery systems reduces the quality of maternity care for most women (Eliason et al., 2013; Requejo et al., 2015). We found that geographic factors arbitrated by rural residence and access (transport/distance) impact prenatal and postnatal care. This corroborates the existing evidence across Ghana that a woman's rural context impacts on maternity and reproductive care utilization (Adanu, 2014; Ayanore \& Aryee, 2015; Overbosch, Nsowah-Nuamah, van den Boom, \& Damnyag, 2004). There is also overwhelming evidence to support these factors as mediators for safe maternity 
care in Ghana and across developing countries (Addai, 2000; Arthur, 2012; Atinga \& Baku, 2013; Babalola \& Fatusi, 2009; Bove, Vala-Haynes, \& Valeggia, 2012b; Simkhada, Teijlingen, Porter, \& Simkhada, 2008). Although regional variations were not observed for rural residence and access factors (transport/distance), older women and widowed/divorced women experienced less or least adequate care. The absence of a female provider was not a problem in seeking maternity care, but it was reported as a significant consideration, with no observed regional variation. However, these women were more likely to be living in rural areas.

Our finding that increasing age and parity are associated with less or least adequate care is well established in several studies (Do et al., 2014; Kodzi et al., 2012; Kruk, Rockers, Mbaruku, Paczkowski, \& Galea, 2010; Ononokpono, Odimegwu, Imasiku, \& Adedini, 2013; Smith, Tawiah, \& Badasu, 2012) as these women are likely to assume confidence in themselves for their own maternity needs. This could be evident in environments where cost and access factors (distance/transport) may create a condition of "despair" and lower seeking behavior for them. Women own say on health was positively associated with least adequate care in this study. This is surprising in the light of health equity campaigns to improve self-autonomy for women health needs. Evidence of poor health care seeking behavior of women attributed to their perceived low selfworth and lack of independence on health-related decisions is reported in most developing countries (Fotso, Ezeh, \& Essendi, 2009; Furuta \& Salway, 2006; Say \& Raine, 2007; Simkhada et al., 2008). Additionally, Eliason et al (2013) affirms that aside autonomy, women personal conviction is important in the utilization of maternal post-partum health services (Eliason et al., 2013a). It may suggest that female autonomy alone may be illusive for improving maternity care if "enabling factors" such as those that provide an effective feedback mechanism for poor access and utilization, and those that seek to economically liberate women are not in congruence with equity and autonomy campaigns. Efforts at addressing the domineering ideological perception of the male spouse as the determinant of womanhood across many socio-cultural, political and social structures are also important. Educational status of women and male partners had no positive association with less and least adequate care in this study.

A limitation to this study is the recall bias as a result of women recount of events during their last birth. The dataset does not provide the possibility to corroborate the women's responses by using data provided by other family relatives or local health professionals. However, our method of cluster analysis provided a good agglomeration of responses which yielded a fair cluster quality on which this study preceded. Since the data were cross-sectional accounts from the 2008 GDHS dataset, causality of prenatal and postnatal care with these associations must be interpreted with care. Due to limitations related to the content of the 
dataset, we also acknowledge that we might have missed other possible factors that have affected women final responses for maternity care use. Also, we might have missed the experience of some women who only had stillbirths or miscarriages in the preceding 5 years because this is a culturally-sensitive topic and such events are usually underreported in surveys. Regional and place of residence variations and trends for maternity care were not explored in this study since we were only interested in segregating results for possible variables providing less and least adequate care for women. Also, the data that we analyzed come from a survey conducted in 2008 which was the only available dataset at the time of analysis. A similar analysis based on new or earlier GDHS waves can provide a base for a comparison across the years.

\subsection{Policy implications for maternity care in Ghana}

Health insurance provides potential benefits for prenatal and postnatal facility utilization especially among rural residents. Improving structural deficits under the current national health insurance scheme will inject more confidence for users, and non-active members who have lost out due to issues of inefficiency and poor quality. We suggest the revision of the insurance type and benefits packages, with target premiums that enable poor households to access not only maternity care, but a continuum of care that indirectly impacts women's health during birth. Current capitation reforms under the health insurance must integrate value-based health care that seeks to provide focused maternity and respond to the general health service need among vulnerable population groups. Additionally, efforts at improving service delivery modes concurrently at the individual, community and facility levels through skilled attendants (physician, midwives, $\mathrm{CHO}$ ) availability, service provision through increased trainings motivation and strict deployment particularly for rural areas are necessary. There is a need to develop and enforce strict policy guidelines and monitoring to ensure skilled personnel equity between rural and urban areas.

A direct policy option to attract and retain skilled personnel to rural areas is needed. Efforts at rolling out the CHPS concept nationwide are long overdue and must be expedited quickly.

Government's policies such as fee exemption for maternity care suffered financial deficits and challenges after initial donor funding from the Dutch government elapsed. Evidence suggests that these policies are often poorly implemented with structural deficits and much political influence that does not create accountability for the beneficiaries (McPake, Brikci, Cometto, Schmidt, \& Araujo, 2011; Ridde \& Morestin, 2011; Singh, Darroch, Vlassoff, Nadeau, 
\& Activities, 2003). We suggest long term financial planning with a coherent maternity care policy plan that creates greater accountability for quality, equity and geography (region and rural residence) where the CHPS system may not have reached.

Lastly, broadening and expanding the opportunities for women beyond having a say on their health needs can help alienate socio-cultural values that devalue women. Incorporating sustained behavior communication strategies into training curricula for health staff will enable community health workers target and design effective maternity adoptive health behaviors at the facility and community. Older and multiparous women need targeting at the community level to improve their urgency for service utilization.

\subsection{Conclusion}

This study re-emphasizes that close to half of women in Ghana experienced less than adequate maternal care considering three aspects of care: prenatal and postnatal care, facility utilization and medication care. Skilled female provider presence enhances adequate care for women at maternity. Across regions and within rural settings, disparities exist, often compounded by supply-related factors. Efforts at addressing skilled workforce shortages, greater accountability for quality and equity, and those that improve women urgency, choices and active participation are important to improve maternity care in Ghana. The cluster methodology in this study has enabled us to segment components of care received. It remains important to design mechanisms to identify those with less and least adequate care use for effective and targeted interventions that improve safe motherhood initiatives in Ghana. It is also important through these mechanisms to identify geographical (regional and place of residence) differences that exist in care levels and groups of women needing safe, effective and respective care.

The study advocates long term planning and systemic reforms on health insurance and maternity care policies. Improving health systems, skilled personnel allocation for care, health staff curricula trainings that recognizes behavior change communication strategies elements for health trainees and broadening women opportunities are vital to the total process of womanhood care beyond 2016. 


\section{GHAPTER 4}

Stakeholders' views on maternity care shortcomings in rural Ghana: An ethnographic study among women, providers, policy makers, and quasi-private sector actors

\section{Draws upon published work:}

Ayanore, MA., Pavlova, M., Biesma, R., \& Groot, W. (2017). Stakeholders' views on maternity care shortcomings in rural Ghana: A qualitative study among women, providers, policy makers, and quasi-private sector actors. The International Journal of Health Planning and Management, DOI: 10.1002/ hpm. 2411 


\section{Abstract}

Introduction: Access to skilled-provider and emergency obstetric care is not universal across all districts in Ghana. The lived experiences of 3 stakeholder groups on maternity care shortcomings in 3 rural Ghanaian districts are examined in this study.

Methods: We applied an ethnographic study approach where field data was collected between March-May 2015 in 3 rural districts of northern Ghana. Data was collected among women with recent births experiences $(n=90)$, health care providers $(n=16)$ and policy actors $(n=6)$. Transcripts were read through to identify similar and divergent stakeholders' views. Significant expressions and experiences of stakeholders on maternity care shortcomings were extracted and evaluated to define key themes.

Results: Four themes emerged: social/community factors, payments for health care, facility level factors and policy level factors. The results show that traditional women's roles divest time for maternity care. Poor transport arrangements, insufficient health workforce, health funding gaps, insurance reimbursements delays, and catastrophic health expenditures on travel and drugs are attested as major barriers across all stakeholder groups in all districts studied.

Conclusions: The discussion of the study findings suggests it is important to ascertain the scale of informal payments and their impacts on health access. Investments in the health workforce and reliable ambulatory service systems could help address poor referral difficulties in rural areas of the country. Social support for community initiatives that pool funds could provide extra resources and relieve cost access-related challenges for using maternity care in rural settings in Ghana. 


\subsection{Introduction}

In Ghana, despite government policies in the last decades to address maternity care barriers, shortcomings for focused maternity care still exist (see Chapter 3). Evidence show a woman's geographical location impacts on access and quality of maternity care utilization in Ghana (GSS et al., 2014). For example, knowledge and awareness of pregnancy complication management varies considerably ( $90 \%$ in urban and 59\% in rural areas for women in reproductive age, 18-49 years) (GSS et al., 2014). Disproportionately, women in the capital (95\% in Greater Accra region) have high foreknowledge of pregnancy complication management issues compared with those in other regions of the country. Across the three northern regions of Ghana, fewer women have focused maternity care compared with urban centers such as Accra (see Chapter 3). Evidence shows less than 10\% of mothers from these three regions receive ANC from a physician (GSS et al., 2014). The 2014 GDHS also reports only 4 in 10 pregnant women (39\%) took intestinal parasite medicines to prevent anemia during their last pregnancy.

Government's policy directives in Ghana over decades have prioritized the role of private health actors in health service delivery. Available evidence in Ghana also shows that when stakeholders feel involved in delivering maternity care services, maternity care service utilization improves (see Chapter 1). This has provided an opportunity for mapping stakeholder interests across several levels: beneficiary, health provider, facility, community leaders, policy, and donor agency level in Ghana. Thematic policy actions, such as the health sector gender policy (2009), the reproductive health strategic plan (2007-2011), and the fee-exemption policy for maternity care (2003), were implemented after consultative stakeholder engagements. Despite these collaborations, health system bottlenecks impact negatively on maternal service delivery and quality (Ganle et al., 2015). Top-down approaches and weak dissemination among policy stakeholders still characterize some health decisions $(\mathrm{MOH}, 2008 \mathrm{~d})$. To assess current service provision and utilization of maternity care, it is important to map key stakeholder views on maternity care shortcomings. This provides an opportunity for building greater consensus to improve women's health outcomes in Ghana.

Earlier studies in Ghana have investigated policy bottlenecks and priority settings for improving safe motherhood interventions (Madi et al., 2007; Rudan et al., 2010; Witter \& Adjei, 2007; Sophie Witter et al., 2007). Other studies assessed the drivers of maternity service utilization (d'Ambruoso, Abbey, \& Hussein, 2005; MOH, 2008; Yakong, Rush, Bassett-Smith, Bottorff, \& Robinson, 2010), whiles others analyzed the views of district level health providers and decision-makers on determinants of maternity service utilization (Banchani \& Tenkorang, 2014; Ganle, 2014; Witter, Garshong, \& Ridde, 2013). Few recent studies in rural 
northern Ghana assessed family relations and health provider's perspectives of utilization barriers for maternity care (Moyer, Adongo, Aborigo, Hodgson, \& Engmann, 2014; Moyer, Adongo, Aborigo, Hodgson, Engmann, et al., 2014; Yakong et al., 2010). None of these studies examined maternity care shortcomings along multiple districts and stakeholder groups.

This chapter aims to examine stakeholder views on maternity care shortcomings in three rural districts of northern Ghana. We jointly mapped and triangulated multiple stakeholder views of women with recent births experiences, health care providers and policy actors in rural Ghana. The aim evolved from the understanding that formal networking and stakeholder interactions are essential for patient- centered maternity care (Jimenez, Klein, Hivon, \& Mason, 2010). We first explored women's views and juxtaposed these with the views of frontline health service providers (midwives/nurses/community health officers) and public and private policy decision actors at regional and district levels. The ethnographic design adopted, enabled a simultaneous reconstructing of lived stakeholders' experiences. This approach enriched the process of understanding stakeholders' constructed meanings and provides another better alternative for improving maternity care shortcomings in poor rural settings.

\subsection{Methods}

\subsubsection{Study design}

To investigate maternity care shortcomings in rural Ghana, we applied an ethnographic study design. Specifically, we explored and compared stakeholders' views on the topic based on an explorative qualitative approach and inductive procedures for data analysis. This study design allows for understanding the phenomenon (in this case, maternity care shortcomings) from the inside (insiders' view) with the support of the etic (outsiders' view) (Atkinson, Delamont, \& Hammersley, 1988; Walsh, 1999). The design approach also assisted to identify the social and cultural sensitivities surrounding the phenomenon from different angles by ensuring 'the voice' of all key stakeholders involved are ensured (Allen, 2011).

\subsubsection{Study setting and ethical approval.}

Geographically, three districts in the Upper East region of northern Ghana (Bongo, Talensi and Nabdam) were purposively selected. These districts have poor indices of maternal birth outcomes out of a total of 13 districts in the Upper 
East region. Proximity and resource availability for data collection also influenced our choice of study districts. A district public health nurse in each district assisted to purposively select 3 remote facilities in each district as study facilities. Thus, 9 district health facilities were included as shown in Table 4.1. All facilities were centers providing maternity and reproductive health services.

Table 4.1: Participant and study setting characteristics

\begin{tabular}{|c|c|}
\hline Stakeholder category/sample & description and number \\
\hline \multicolumn{2}{|l|}{ Women participants $(n=90)$} \\
\hline Place of giving birth & home (13), facility (77) \\
\hline Participant age (range) & $18-35$ years \\
\hline Educational status & never educated (69), ever educated (21) \\
\hline Residence type per community(s) & $\begin{array}{l}\text { Rural (Namoo, Boko, Amanga, Korogo-Kaare, } \\
\text { Yakote, Winkogo, Tongo, Kongo-central, Nango- } \\
\text { di-central. }\end{array}$ \\
\hline Married Status & married (81), divorced/widowed (9) \\
\hline Employment status & unemployed (64), self-employed (26) \\
\hline Bongo participants & FGDs ${ }^{\mathrm{a}}-30$ women \\
\hline Talensi participants & FGDs-30 women \\
\hline Nabdam participants & FGDs-30 women \\
\hline \multicolumn{2}{|l|}{ Facility description/number per district } \\
\hline Bongo & 2 CHPS $^{\mathrm{b}}$ centers, 1 health center \\
\hline Talensi & 2 health centers, 1 CHPS center \\
\hline Nabdam & 2 health centers, 1 CHPS center \\
\hline \multicolumn{2}{|l|}{ Health care providers $(n=16)$} \\
\hline Bongo district & FGDs-3 persons \\
\hline Talensi district & FGDs-6 persons \\
\hline Nabdam district & FGDs-7 persons \\
\hline \multicolumn{2}{|l|}{ Policy decision actors $(n=6)$} \\
\hline Regional level (public policy actors) & IDIs'-1 person \\
\hline District level (public policy actors) & IDIs-3 persons \\
\hline $\begin{array}{l}\text { Quasi-private health managers (private } \\
\text { policy actors) }\end{array}$ & IDIs-2 persons \\
\hline Total participants & 112 \\
\hline
\end{tabular}

FGDs $^{\mathrm{a}}=$ focus group discussion, CHPS $^{\mathrm{b}}$-community-based health planning and services-(lowest health delivery unit at the community level), IDIs ${ }^{\mathfrak{c}}=$ in-depth interviews.

These facilities were selected because they provided services along a large coverage area and because the possibility to attain saturation from study respondents was possible. The Upper East regional capital, Bolgatanga, was included as a study 
setting since public and private policy actors resided in this setting. Study approval was granted by the Institutional Review Board of the Navrongo Health research Centre, Ghana (ID: NHRCIRB202). All eligible participants who accepted to participate duly signed/thump printed informed consent forms and educated on steps to take to withdraw from the study.

\subsubsection{Data collection and sampling procedures}

Data collection was carried out between March and May 2015. Three stakeholder groups were included in the study: women who had given birth in the preceding 2 years prior to study, facility staff members, and policy actors. Two instruments for data collection were used; FGDs applied with women and health staff, and IDIs with policy actors. To select health staff participants, all 9 district study facilities were visited to engage the health staff on the study aim. From the three facilities in each district, one facility was chosen as a main district study facility. In each main district study facility, one midwife, one senior staff member (facility in-charge), and facility nurses (average 3 to 7 persons per district), responsible for providing maternity and reproductive health services were purposively recruited as district health staff participants (see Table 4.1).

The researcher requested midwives in all 9 district study facilities to provide a listing profile of women aged 18-35 with a recent birth experience ( $<24$ months), from the antenatal and postnatal facility registers ( 15 women per facility). Thus, in total 135 women were purposively selected as potential eligible participants for the study. In 2 districts; Bongo and Talensi, all initially profiled women were visited to assess their inclusion in the study. In the third district, Nabdam, 6 initially profiled women were absent and were excluded from the study. They were replaced by other 6 eligible participants from the district facility antenatal registers. At each district level, a final inclusion checklist for women was used; woman should have been physical present, consent to participate, interested in the study and able to share prior knowledge related to the study aim. A final convenient sample of 30 eligible women per district took part in the FGDs (3 FGD per district with 10 women in each group discussion). Thus, a total of 90 eligible women discussants took part in the study. The rest of the women, who were in the initial list but not included in the study, were asked to be available to cater for any dropouts. No dropout to participate was recorded.

Policy actors were the last group of stakeholders recruited for the study. Policy actors (public policy makers and quasi-private sector actors) from each study district and regional capital were recruited to participate. The purposively selected participants in this group included 2 private policy implementers and 4 public policy actors at regional and district levels. The choice, availability and 
contribution of selected stakeholders informed their inclusion in study. Women FGDs were conducted in Tali, Nabit and Grune languages. Interviews with health staff and policy actors were conducted in English. Sample FGDs and IDIs questions are presented in Figure 4.1.

\subsubsection{Data Analysis}

Audio recordings from FGDs and IDIs were transcribed verbatim. NonEnglish audio recordings from women FGDs were transcribed by native speakers of the three languages. An inductive thematic analysis procedure was followed in the data analysis process. Specifically, all transcripts were repeatedly read by research team to identify similar and divergent views in the data. The process of 'extracting significant statements' (Colaizzi, 1978) enabled us to ascribe meanings to the data collected. Thus, the process of 'extraction' resulted in the formulation of sub-themes per stakeholder group based on the data. Further inductions and verification were conducted leading to the emergence of 4 final themes. Resulting inductive emerging themes were sorted into a framework showing how final themes emerged (see Table 4.2). Descriptions of final themes are presented in quotes, drawing consensus and inferences across all stakeholders.

\subsection{Results}

Socio-demographically, women's parity levels ranged between 1 to 7 births. Of all 90 women participants, 4 women reported previous pregnancy terminations. A total of 13 women ever had home births. Health staffs were comprised of 3 midwives, 3 senior health staff and 10 community/enrolled nurses with various years of working experience with the Ghana Health Service. All 6 policy actors (public/private) on average had a minimum of 8-15 years in health policy implementation in Ghana. Quasi-private policy actors were 2 while the remaining 4 participants worked under the Ghana Health service strature.

The inductive analysis generated 4 themes; socio-cultural and communityrelated shortcomings, health facility level factors, informal related payment factors, and health policy views for maternity shortcomings. The sub-sections examine each of these theme categories in Table 4.2 into detail. 


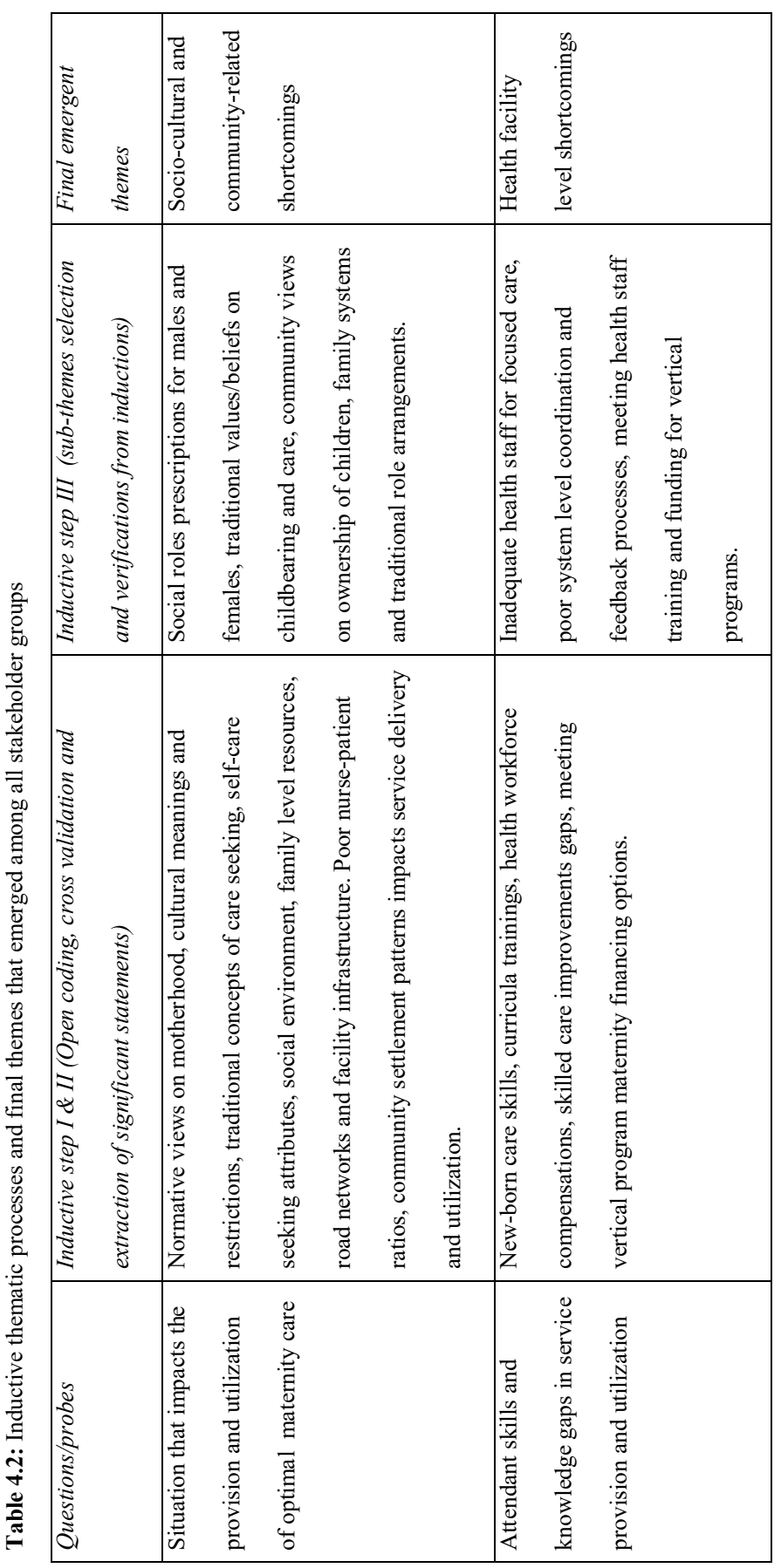




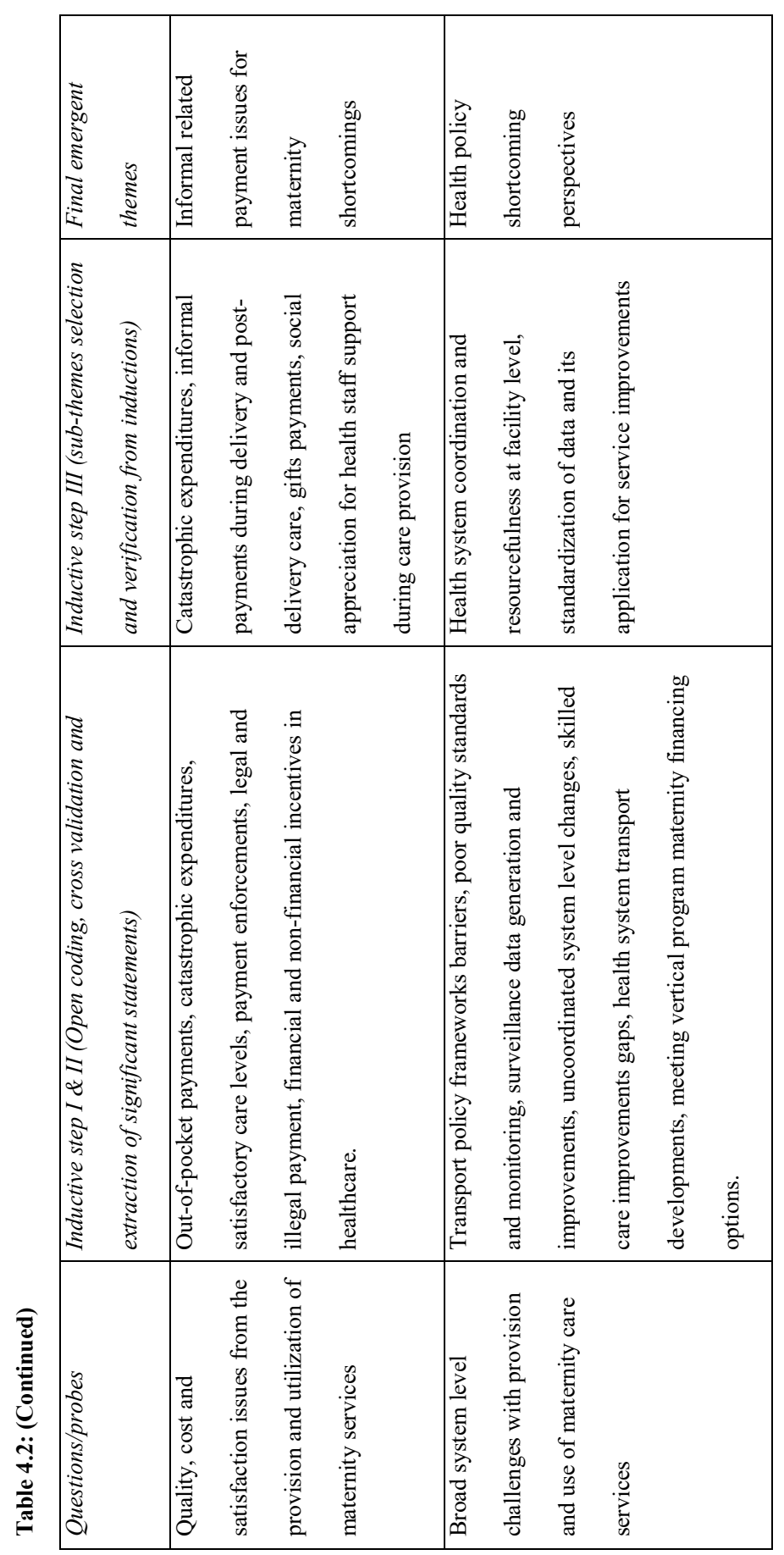




\subsubsection{Emergent Theme I: Socio-cultural and community-related perspectives for} maternity shortcomings

The majority $(n=68)$ of women decisions to seek maternity care were influenced by the perception of their families abetting travel distance, time and cost barriers for care. Women attested that their poor accessibility to health centers was also due to poor road networks linking between their communities with facility centers. The average distance from the community centers to facility levels was $18 \mathrm{~km}$. This phenomenon was exacerbated by dispersed community settlements away from health centers. These physical barriers for accessibility accounted for home births asserted by one participant during FGDs as presented in theme I of Box 4.1.

Dispersed settlement patterns are viewed by health providers and policy actors to account partially for physical access difficulties challenges cited by women. Policy actors affirmed migration as a major social challenge at the community level. One of the policy actors emphasized: "We cannot chase people with health services everywhere". According to the policy actors, the creation of new settlements further away from growing towns and communities makes health care delivery more expensive and less accessible. Within the socio-cultural environment in study districts, salient cultural prestige for home births was expressed by women as desirable. However, this cultural prestige is not often spoken about as women shy away to protect their relations with clinic staff. Few women who gave birth at home recollected the social and cultural prestige they enjoyed with their husbands after a home birth. More so, high parity desires by some women and their families resulted in women skipping vital health services such as ANC in order not to be recognized by the health care system. Such women either attended ANC and skipped facility-based birth care, or avoided antenatal and facility-based birth care and only attended postnatal care services.

Additionally, women reported that traditional triple roles in meeting reproductive, productive and community expectations put huge competing demands for seeking maternity care. Women avowed competing time demands at the household level to meet domestic and social expectation causes time delays for antenatal and postpartum family planning sessions. Health providers agreed with women's limited self-autonomy in seeking care and were categorical about the inability of some women to consistently meet the recommended follow-ups during pregnancy and after childbirth. 


\subsubsection{Emergent Theme II: Health facility level factors for maternity shortcomings}

Strong views on health provider neglect during facility visitations were catalogued by some women in Bongo and Talensi districts, but however not reported by women discussants in Nabdam district. Also, noisy birth-rooms attributed to limited birth-room space and close proximity to outpatient centers were reported by some women to affect their privacy during birthing. Prolonged waiting times and uncoordinated arrangements at facility centers take a lot of women's productive time, which sometimes discourages women to seek care. Policy actors acknowledged meeting women's demands and expectations remains a major barrier because of current staff shortfalls and the need to meet patient-centered care services. All stakeholder groups agreed to the difficulty for individualized focused care due to the poor patient-nurse ratios. Policy actors indicated some health staff refusal to work in less resourced areas (mostly rural facilities) accounted for skilled personnel shortfalls in certain rural facilities. Both policy actors and health providers contended the phenomenon is created by poor policy strategies to address the needs of skilled staff willing to work in rural facilities. Private policy actors spoke of bottlenecks in the selection and training of health staff, uneven distributions across private and public sectors, and poor workforce arrangements at rural facility settings.

Meeting demands for care for the mother and child beyond the childbirth remains problematic. Women bemoaned the logic of promoting ANC and facilitybased births without active follow-up checks after they deliver at the facility, something they admit would be useful in sustaining many good outcomes for them and their babies within the first six months after birth. Both health providers and policy actors admitted gaps in the overall monitoring of the care provided, especially when home visits are needed. Public and private actors agreed with the existence of systemic gaps in the knowledge of the health staff and with the poor travel logistics that prevented health staff to undertake home care visits.

More than half of health staff across all districts agreed to wide assertions of insufficient skills training and its effects on staff skills set for new-born care. Health staff views on newborn care skills admitted other trainings are often regular and depended on low budgetary allocations, often stifling the ability to engage a large number of health staff. These views are reflected in health provider assertions presented in theme II of Box 4.1.

Discussant views of all stakeholder groups admitted poor referral transport arrangements impacted negatively on women ability to meet referral needs for obstetric care. Women openly complained about discomforts caused by the use of motor tricycles as referral transport systems at some facilities. Some women were 
asked to pay cash to cover fuel expenses since facilities did not have the resources to cover this and other related expense as one participant highlights under theme II of Box 4.1.

Private health actors impugned the state of the country's national ambulance system and wondered why patients should pay for such services. They argued that the current maternity service provision is chaotic and cannot effectively support women in meeting their needs of maternity care.

From the above expressions, both public and private policy actors acknowledged the poor coordination and institutional linkages between the national ambulance system and the health care delivery system at regional, district and primary facility level. The need for effective coordination was repeatedly asserted by all stakeholder groups as key for ensuring maternity referral inefficiencies for most rural women are improved.

\subsubsection{Emergent Theme III: Informal related payment issues for maternity shortcomings}

Catastrophic expenditure on drugs not covered under the national health insurance is expressed as a barrier to maternity health seeking by women in this study. Besides direct payments for drugs, indirect travel cost to acquire drugs remains problematic for some women and their households. Other indirect expenditures, such as preparing for a facility-based birth, have been indicated by the majority of women as critical when deciding on the place of giving birth. Separate interviews with women with a history of home births show major decisions such as facility level expectations and demands prior to women birthing at a facility level influenced their choice for home birth as recounted by one participant accounts captured under theme III of Box 4.1.

Eight (8) women reported home births due to their inability to meet the strict number of soap demands from facility level health staff ( 8 from the total of 13 home births in the study). Policy actors emphasized that midwives were expected to follow strict compliance with the regulations for infection control and prevention (IPC). Thus, women are expected to meet the minimum standards for facilitybased births since the health care system cannot always provide the necessities. Policy actors indicated that it is illegal for health staff to demand gifts or payments from the women before providing the needed care. Health staff referred to these situational issues as a pure gift with no effects on their provision of services to women. However, some women reported that gifts to health staff from women are detrimental for the overall treatments and care received. Gifts such as soap and food are reported as common. Although the majority of the women discussants acknowledged that their gift giving was voluntary, their decision to provide gifts 
were largely influenced by their own previous experience or that of other women showing that gifts giving often yield friendlier and quicker services at the facility level.

These expressions by women were captured amply by two FGD participants views presented under theme III of Box 4.1.

Women referred to this as "gift assurance" in meeting facility-based care needs. These gift payments expressed by some women were observed to influence care quality received and their relations with healthcare providers. Close to half of policy actors admitted that gift giving by women was culturally ingrained and could potentially affect vulnerable women if the health system does not find a way to eliminate such barriers.

\subsubsection{Emergent Theme IV: Health policy perspectives on maternity shortcomings}

At the health policy level, current inefficiencies in funding gaps of the national health insurance scheme is seen by the policy actors as a potential threat to future quality maternity care. Irregular reimbursements to health facilities by the national health insurance institution affect the drug and other supplies at the facility level. Health staff in our study agreed with the women's claims about drug shortages, emphasizing supply side problems. Both public and private policy actors agreed that the national health insurance challenges negatively affect the service quality and create distrust in the health care system among the care seekers. Private policy actors were emphatic of how some private health facilities often withdrew or withheld general health care provision due to late or poor reimbursements from the national health insurance scheme as recounted by one policy actor view presented under theme IV of Box 4.1.

According to policy actors in this study, poor health data gathering and storage at the district and facility level hampers effective decision-making and impedes quality improvements in maternity care. The unavailability of reliable health data negatively affects planning and resource allocations at the district, regional and national level. Even among the health facilities with good management information systems, linking data to service care is problematic due to insufficient support to generate reliable health system data at both the public and private service provision levels. These consensual views are presented under theme IV of Box 4.1.

Policy actors acknowledged the low emphases on funding for health research. They assert policy formulation sometime lacks appropriate linkages with context based evidence, thus the existence of policy formulation and implementation gaps. 
Poor budget allocations hamper the health staff ability to implement evidencedbased strategies that can effectively improve maternity care utilization.

Policy actors noted the non-participation and inability of other relevant stakeholders to contribute to policy decisions due to the low level of knowledge and skills related to policy formulation and dissemination at a local level.

Box 4.1: Final emergent theme categories among all stakeholder groups from the study

\begin{tabular}{|c|c|c|c|c|}
\hline Themes & $\begin{array}{l}\text { Theme I: Socio- } \\
\text { cultural and } \\
\text { community-related } \\
\text { perspectives on } \\
\text { maternity care } \\
\text { shortcomings }\end{array}$ & $\begin{array}{l}\text { Theme II: Health facility } \\
\text { level factors for maternity } \\
\text { care shortcomings }\end{array}$ & $\begin{array}{l}\text { Theme III: Informal } \\
\text { related payment issues } \\
\text { of maternity care }\end{array}$ & $\begin{array}{l}\text { Theme IV: Health policy } \\
\text { perspectives on maternity } \\
\text { care shortcomings }\end{array}$ \\
\hline & $\begin{array}{l}\text { I delivered at home } \\
\text { because we were not } \\
\text { able to organize a } \\
\text { reliable transport } \\
\text { when labour } \\
\text { contractions started } \\
\text { at home (FGD } \\
\text { participant at } \\
\text { health centre- } \\
\text { Nabdam } \\
\text { Our community is } \\
\text { far and the roads are } \\
\text { bad during the } \\
\text { raining seasons } \\
\text { (FGD participant } \\
\text { at heath centre- } \\
\text { Bongo) } \\
\text { We cannot chase } \\
\text { people with health } \\
\text { services everywhere } \\
\text { if seasonal } \\
\text { community } \\
\text { migrations are not } \\
\text { controlled } \\
\text { (Consensual views } \\
\text { of policy and } \\
\text { health staff } \\
\text { participants) }\end{array}$ & $\begin{array}{l}\text { We don't know everything } \\
\text { on new-born care. Recent } \\
\text { trainings have helped, but } \\
\text { are not adequate. More } \\
\text { trainings for health staffs on } \\
\text { collaborative health roles to } \\
\text { improve maternity care will } \\
\text { be helpful (Health } \\
\text { participant, Talensi) } \\
\text { When I was pregnant for my } \\
\text { third child, I visited the } \\
\text { facility and after two hours I } \\
\text { could not give birth, the } \\
\text { facility demanded I pay for } \\
\text { fuel so that another available } \\
\text { health facility vehicle } \\
\text { transport me to the } \\
\text { Bolgatanga regional } \\
\text { hospital...my husband had to } \\
\text { pay } 40 \text { Ghana cedi before I } \\
\text { was transported to the } \\
\text { regional hospital (FGD } \\
\text { participant, Talensi) }\end{array}$ & $\begin{array}{l}\text {...that is not my first } \\
\text { time delivery. They kept } \\
\text { insisting on items that } \\
\text { should be brought } \\
\text { anytime my delivery } \\
\text { date is due... I had to } \\
\text { deliver at home because } \\
\text { I cannot afford the } \\
\text { delivery items expected } \\
\text { at the health } \\
\text { facility...(IDI } \\
\text { participant, Talensi) }\end{array}$ & $\begin{array}{l}\text { We almost withdrew our } \\
\text { services for NHIS card } \\
\text { holders a few months ago, } \\
\text { because we could no longer } \\
\text { pay our suppliers for drugs } \\
\text { and other essential } \\
\text { equipment's to keep our } \\
\text { facilities running. These } \\
\text { delays are really worrisome. } \\
\text { We need to have a national } \\
\text { consensus on future health } \\
\text { funding issues (Regional } \\
\text { level private policy } \\
\text { participant) } \\
\text { We rarely make use of any } \\
\text { data we collect at the facility } \\
\text { level. Improvements will } \\
\text { only come if these data and } \\
\text { information are used for } \\
\text { more planning for the } \\
\text { general improvements within } \\
\text { the health care system } \\
\text { (Consensual view of all } \\
\text { policy participants) }\end{array}$ \\
\hline
\end{tabular}

\subsection{Discussion}

This study analyzed views among three key stakeholder groups (women, care providers and policy decision actors) on maternity care shortcomings in rural Ghana. Our findings reflect a broad range of factors of maternity care shortcomings, namely factors related to social arrangements in the community, health system (payments and provision of maternity care) and health policy frameworks for service provision. Some of the views expressed by health providers and policy actors on reasons for care shortcomings differed from those of women lived experiences. For example, policy actors and care providers were of the view that 
physical access difficulties for maternity care are driven more by poor settlement patterns and social norms. Women on the other hand gave priority to travel time, direct and in-direct cost barriers and poor facility level conditions as major determinants to care seeking. Additionally, women experiences of poor facility level conditions, poor focused care, and inhumane treatments were impugned to health staff perception of their low socio-economic status and backgrounds. However, health staff and policy actor concerns on poor focused care asserted by women were more driven by inadequate staff and logistical provisions at the facility level, making it difficult to deal with high patient numbers and referral care. Differences in opinions between women and health staff on maternity care shortcomings enumerated above is observed in other studies in Ghana as well (Halley Crissman et al., 2013; Witter, Arhinful, Kusi, \& Zakariah-Akoto, 2007), highlighting that stakeholders may differ in opinions on reasons for current existing barriers to adequate care. A potential consequence of the above could be a decline in stakeholder trust and a negative impact on women's use of services ( $\mathrm{MOH}, 2008)$. To address this nascent issue, care providers long held traditional models must evolve towards a more patient centred care and more shared decision making model. There is a need for effective communication and dissemination of care standards addressing myths of standards of care at various stakeholder levels.

Beyond these differences in stakeholder opinions, many shortcomings put forward in this study reflect consensual views. These views refer to access barriers such as poor roads and poor-facility transport referral systems, which are well known in Ghana (Asenso-Okyere et al., 1998; Buor, 2003; Overbosch et al., 2004; Pell et al., 2013). The inability for women to own household resources have a negative impact on their ability to meet financial constraints for health care access in Ghana (Atinga \& Baku, 2013; Dixon, Tenkorang, Luginaah, Kuuire, \& Boateng, 2014; $\mathrm{MOH}, 2008$ ). Our findings show maternity policy strategies such as the freefee delivery policy in Ghana has not been able to tackle indirect cost associations for maternity care. Studies in other countries also evidenced women recounting crossing rivers, uncomfortable motor tricycles used as transport, and poor road infrastructure (Mathole, Lindmark, Majoko, \& Ahlberg, 2004; Mumtaz \& Salway, 2005). To improve the standards of maternity care, social investments in roads and reliable ambulance referral systems as well as investments that meet logistical and staff capacity building needs centered on newborn care remain vital (Blas et al., 2008; Marmot et al., 2008). There is also an urgent need of increased government funding to avoid shortages of drugs and medical supplies (Jo Borghi et al., 2006). Adequate funding to tackle indirect cost effects for patient care could help reduce catastrophic payment by households at the point of maternity service use.

Women's care seeking behavior is also influenced by male and family relations that often take away women's choices and time for seeking maternity care (as 
indicated by the women in this study). This socio-cultural problem is reported in other studies in Ghana (Addai, 2000; Withers et al., 2013) and in other African countries (Babalola \& Fatusi, 2009; Somé et al., 2013). In many communities across Africa, normative factors play a vital role in influencing maternity care utilization for women. A new insight provided by our study for rural Ghana is related to the statements of some women that they skipped antenatal and birth care because of embarrassment on their parity levels. This insight revealed by women and health staff is attributed to emerging contentions between salient cultural demands on women and increasing family planning messages received by women during antenatal visits. These assertions were made by women whose spouse desires many children as an act of gratification and social fulfillment. Women in such family settings often lack knowledge about the risk associated with their intentions on avoiding maternal care. This finding is similar to a study among Indian women who shyed away from health staff in order to hide their parity desires from clinic staff (Devasenapathy et al., 2014). Additionally, among economically empowered women, the burden of economic, child caring and reproductive role expectations for women may cause delays for maternity care utilization.

All stakeholders in our study admitted that knowledge and skills for both mother and new-born care is a determinant for women trust and use of facility birthing services. Women in group discussions openly made reference to periods when they experienced poor services under the support of health staff they perceived as having poor skill knowledge. This finding is also widely documented in other studies (d'Ambruoso et al., 2005; UNFPA, 1999). Women interviews showed their conscious desire to be attended to and receive care by professional midwives, irrespective of the distance. Evidenced were women wide held views that they would often walk long distances to further away facilities if their local facilities lacked skilled health personnel. To address these gaps, continuous skill and professional job training to improve clinical competences of midwives and other supporting facility level staff is important. There is also a need for districtwide health monitoring systems that ensures patient complaints on delivery standards are redressed to restore confidence and low care provision perceptions among users of maternity care services.

Poor bedside manners and delivery room environments (including noisy delivery room environments) in some facilities add to the frustration and poor quality of delivery care asserted by some women. This findings support evidence on poor delivery environments and health system barriers in Ghana (Ganle, 2014; $\mathrm{MOH}, 2014$ ). To address poor delivery rooms, low privacy and other poor facility environmental conditions, there is a need for facility level improvements to guarantee that women receive services in environments that 
supports their willingness to visit the facility for antenatal and delivery care. In addition, deficits in the communication between the midwife and women-patient impacts negatively on standards and timing of care provided and received by women in this study. This phenomenon, admitted by all stakeholder accounts for poor focused care as health staff are overburdened, with often reported episodes of health staff unfriendliness. Similar evidence from this study finding is documented (d'Ambruoso et al., 2005; Devasenapathy et al., 2014; Larson, Hermosilla, Kimweri, Mbaruku, \& Kruk, 2014; WHO, 2014). Improvements in these areas need multiple targeted responses. Educational curricula in health institutions should be broadened to include anthropological and social drivers of care giving. Other strategies could include performance-based facility financing and contracting schemes to motivate health workforce to serve in deprived rural settings (WHO, 2010).

Within the policy framework for improving maternity care, the culture of "gift assurance" as coined by women requires policy control measures to avert catastrophic health cost for vulnerable households. Existing evidence in Ghana show gift giving by women could exacerbates further informal payments for facility delivery care (Witter, Garshong, \& Ridde, 2013). Although women in our study acknowledged this phenomenon did not exist in all facilities, their existence and potential impact on women later care seeking needs could be better addressed with a policy culture that demands greater accountability at facility levels. As pointed out by health providers and some policy actors, data on patient's history is hardly fed into the health care strategy and facility level improvements. Recent use of facility computer systems to ensure health insurance reimbursements has however helped to improve this. As agreed by providers and policy actors in our study, poor data and surveillance systems negatively impact the delivery of integrated maternity care. This is characteristic for many settings outside highincome countries (Benach, Muntaner, Santana, \& Chairs, 2007; Sophie Witter et al., 2007). To meet this challenge for maternity service delivery, investments in reliable, cost-effective data systems at facility level are important. There is need to train district health information officers to effectively supervise and facilitate the collection and processing of data to inform quality service provision and utilization at facility levels. The need for such effective systems is widely supported as important not only to guarantee health system improvements but to enhance health equity dimensions (Bonnefoy et al., 2007), especially in rural areas.

Despite the saturation observed at the end of the data collection processes, we may have still missed out categories that could have emerged in other study settings. Recall bias by women participants regarding their past childbirth events is possible although studies on childbirth evinced the women's ability to recall birthing experience rightly (d'Ambruoso et al., 2005; Simkin, 1991; Tautz, Jahn, 
Molokomme, \& Görgen, 2000). Providers and policy actor's bias in trying to hide shortcomings is also possible. This provider related bias is however minimized since a triangulation with women's views is applied.

\subsection{Conclusion}

The factors impacting maternity care in rural Ghana are multifaceted and complex, operating at household, community, health system and policy formulation/coordination levels. Our results indicate stakeholder differences and consensuses on key shortcomings for maternity care in rural Ghana. This study highlights the need to strengthen not only institutional care infrastructure, but improve social conditions and facilitators for care seeking at the facility level. Differences in opinion between women, health staff and policy actors are not new in Ghana. Our findings advocate for inclusiveness and the effective dissemination of patient care delivery standards among women and health care staff at all levels. This has the potential to create a culture of trust, transparency and openness regarding ways to tackle barriers for adequate care provision and seeking. At the individual level, patient-staff conditions that create a culture of trust and respect will contribute to patient-centered care and address traditional roles and social norms in birthing practices. At the policy level, targeted investments in increasing professional skill numbers in newborn care and poor delivery room conditions could improve staff competencies to meet inadequate conditions of care quality at facility levels. In pursuit of these, a strong political environment to improve rural road infrastructure, and to take steps to ensure a more effective decentralized national ambulatory service system will better ensure women effectively benefit from the country's free maternity care policy. To improve women care seeking conditions, pilot programs in health facility financing and contracting schemes could help address current health sector funding gaps, motivate health staff to work in rural areas and support address informal payment complaints by women in meeting rural maternity care needs. 


\section{CHAPTER 5 \\ Context-specific factors and contraceptive use: accounts of women, men and health providers in a rural Ghanaian district}

Draws upon work under review:

Ayanore, MA., Pavlova, M., \& Groot, W. (2016). Context-specific factors and contraceptive use: accounts of women, men and health providers in two rural Ghanaian districts. 


\begin{abstract}
Introduction: Suitable options for improving women's access to effective, safe and context-specific contraceptive methods must be explored to curtail stalled or rising unmet needs in contraceptive use in rural Ghana. The Chapter aims to outline specific factors associated with contraceptive use, access on demand and future use intentions among women in two districts of Ghana.

Methods: Using a descriptive cross-sectional design, quantitative data $(n=720)$ were collected among women aged 18-28. Focus group discussions and in-depth interviews were also conducted among women $(n=30)$ aged 18-49 and men $(n=10)$ respectively. IDIs were conducted among 3 midwives.

Results: Focused counselling is vital for contraceptive use. Male partner support can drive cultural sensitivities towards accepting use of contraception. Covert use is still preferred by most women in the study. Services delivered on good provider-relational grounds and convenient clinic hours encourage contraceptive use among women. Health care providers who address individual misconceptions and confidentiality significantly, contribute to contraceptive use. Counselling services for contraceptive use is vital for rural contraceptive use. Male targeting to improve contraceptive service use must first identify the specific preferences of the woman, since covert use is highly appreciated.
\end{abstract}

Conclusions: Ascertaining the prevalence of covert use and how community systems can address this for improved contraceptive uptake is further recommended. 


\subsection{Introduction}

Despite the increase in global contraceptive use, millions of women of reproductive age (15-49 years) have unmet needs for contraception (WHO, 2015). Method related side effects, partner opposition for contraceptive use, financial constraints to access and poor provider-client counselling needs are often cited as barriers to contraceptive use (Casterline \& Sinding, 2000). User misinformation on contraceptive use and medical barriers also account for lower contraceptive uptake worldwide (Ashford, 2003; Jacqueline Darroch \& Singh, 2013; UNICEF $\&$ WHO, 2012). These barriers affecting women's choices for fertility needs are described as "remarkably pervasive" (Campbell, Sahin-Hodoglugil, \& Potts, 2006). Barriers affecting women's choices are also increasingly evidenced to affect women's ability to effectively regulate their reproductive choices in the short to long term (Asekun-Olarinmoye et al., 2013; Campbell et al., 2006; Smith, Ashford, Gribble, \& Clifton, 2009). Unintended pregnancies and unsafe abortions are the consequences of unmet contraceptive needs in most developing countries (see Chapter 2). Consequently, when faced with an unintended pregnancy, women may resort to an unsafe abortion if adequate safe abortion services are not available (Campbell et al., 2006; Mosha, Ruben, \& Kakoko, 2013; Sedgh et al., 2012).

In Ghana, unmet need levels for family planning stands at 29.9\% (see Chapter 1). Abortion in Ghana has been asserted to be widespread (Blanc \& Grey, 2002) too, underpinning research findings that women contraceptive demands are not fully met among certain population groups (Johnson \& Madise, 2011). Ineffective use of modern contraceptives is also reported among women in Ghana (Adanu et al., 2009; Asamoah, Agardh, \& Ostergren, 2013; Joseph Kofi, 2013). Evidence of trends of modern contraceptive uptake differentials between urban-rural areas is known (GSS et al., 2014; GSS et al., 2009). An assessment of barriers and determinants for family planning uptake show context differences exist across and within regions. Religion, place of residence (rural/urban), educational status, age, parity and social economic status are among common predictors of family planning use in different settings. Many studies in Ghana have provided wide- range predictors and determinants of family planning use across several population groups (Abekah-Nkrumah \& Abor, 2016; Atuahene, Afari, Adjuik, \& Obed, 2016; Blanc, 2001; Dalaba et al., 2016; Eliason et al., 2013). However, studies in SSA and West Africa show that different factors influence fertility and family planning interventions differently (see Chapter 2). This underscores the need for identifying effective context family planning factors as an important step for improving acceptance and uptake of such services in many local contexts in Ghana. Across northern Ghana where this study was conducted, fertility studies on the use, barriers, and influencing factors related to contraceptive use have 
largely relied on the DHS data. Other studies have focused on the use, sociocultural determinants, and barriers for women contraceptive utilization (Adongo et al., 1997; Halley Crissman et al., 2012; Debpuur et al., 2002; Yakong et al., 2010). While prevailing evidence on women's low or non-use of contraceptives exists widely in some districts of Ghana, limited knowledge of context drivers for contraceptive use exist in the Garu-Tempane district of Ghana. The district remain largely rural, and dominated by poor indices of low acceptance rates of contraceptive use compared with other districts across the region.

This chapter aims to explore specific factors associated with contraceptive use among women in the Garu-Tempane district of Ghana. Three main measures were investigated; current contraceptive use, access on demand for contraceptives, and future use intentions for contraceptive use. The study was necessitated due to poor data on family planning practices and use in the district. It was also necessary to provide large scale baseline data to district health teams working to scale up free family planning services across the district. Understanding women's accounts and choices for contraceptive use could provide acumens for improved contraceptive uptake in the districts. Such evidence is needed to inform district, regional and national health planning programs for family planning interventions. Our study specifically contributes to the literature by exhuming knowledge related to rural context-specific factors influencing contraceptive uptake. Characterized by a study setting typical in rural SSA, the study provides insights into rural context factors that need attention to accelerate progress for women contraceptive use in rural Ghana and in other rural settings in SSA.

\subsection{Methods}

\subsubsection{Study setting}

Data for this study were collected in the Garu-Tempane district in the Upper East Region of Ghana between February and March 2012. The Garu-Tempane district is located in the south eastern corner of the Upper East Region of Ghana. The 2010 population census in Ghana gave an estimated population of 130,003 inhabitants, comprising of 62,025 males (47.9\%) and 67,978 females (52.3\%). An estimated $95 \%$ live in rural areas, with a youthful population $(46.0 \%)$. Fertility preferences across both districts show an average fertility rate of 3.9, with an estimated general fertility rate of 106.2 births per 1000 women aged 15-49 years (GSS, 2012). The district is inhabited by many tribes; Bimobas, Kusasis, and other minority tribes such as Busangas, Mamprusis and Fulani's. At the time of the study there were 24 functional health facilities zoned under 6 sub-districts managed by 
the district health administration in the two districts. The 6 sub-district health zones served 194 communities within the district. Earlier evidence in the district show that the majority of girls have their first birth before age 18 (Meij, De Craen, Agana, Plug, \& Westendorp, 2009). The study population included young women (18-28 years), women of reproductive age (15-49 years), male spouses and midwives.

The study employed a descriptive cross-sectional design. All 24 functional health facilities within the Garu- Tempane district at the time of the study were conveniently included. The health facilities included 6 health centers, 4 clinics, 12 Community Based Health Planning and Sservice (CHPS) centers and 2 privately health facilities. The Garu township had a total of 8 health facilities whiles 17 of the other facilities were located in 17 different communities across the district jurisdiction. 2 main phases of data collection were simultaneously carried out: quantitative and qualitative phase. The phases are subsequently described together with the methods of data analysis applied.

\subsubsection{Quantitative phase}

The quantitative part of the study took the form of a survey among young women aged 18-28. The sample size for the survey was determined using Leslie Kish (1986) formula for survey sampling for descriptive studies (Kish, 1986). The required sample size was estimated to be 316 participants. To select the participants, maximum variation purposive sampling was applied where 30 eligible women seeking healthcare were sampled across all 24 functional health facilities selected for the study. A total of 720 survey questionnaires were administered in all 24 health facilities.

Survey participants were recruited while waiting in queues to consult health staff at one of the 24 health facilities. At each health facility, 30 young women were purposively selected as eligible participants. Women were eligible if they were mothers, within the age 18-28 and received family planning and reproductive health services within the last 6 months in any facility within the study settings. For women who could not recount their birth dates, their personal clinic records were assessed to ascertain this. The study purpose was described to each eligible participant. A verbal consent of the participant was requested. There were no refusals. Participants, who provided such consent, were invited to participate in the survey after they were attended to by the health staff. Women reported on a rolling basis to the research team after receiving health care services. The data collection at a given facility ended after the 30th participant was recruited. The survey questionnaire was pre-tested twice at the Garu health center to validate its appropriateness for data collection (face validity). Questionnaires 
were administered face-to-face with participants with the support of six research assistants recruited across the six zones in the district. Three languages; Kusal, Bimob and Bissa were adopted to be used in collecting survey data across all facility settings. Each research assistant had adequate knowledge of these three dominant languages for administering the questionnaires.

The survey questionnaire included 15 questions on current and ever use of contraceptives, information and contraceptive decision making, support for contraceptive use, and costs associated with contraceptive use. In addition, there were 10 questions that assessed access on demand for contraception and women's intention for future contraceptive use (See Appendix D on sample wording of questions). Field editing on questionnaires was performed on a daily basis throughout the period of data collection. This ensured that all entries for respondents were properly tabulated prior to the data entry. A total of 16 questionnaires were identified as poorly filled out. These questionnaires were screened and follow-up visits to respondents was undertaken to ensure the proper fill out of the questionnaires. Additionally, the six research assistants who supported the data collection cross validated each other's work daily. Final data cleaning was performed by the researcher. Data were entered into SPSS version 22, cleaned and analyzed. Descriptive statistics were estimated for all sociodemographics applied in the study. Multinomial logistic analyses generated odds ratios to show significant associations between socio-demographic and health characteristics, and the three key measures in the study: contraceptive use access on demand, and future use intentions.

\subsubsection{Qualitative phase}

FGDs and IDIs were applied for the qualitative data collection. The FGDs were designed to engaged additional women $(n=30)$ aged 15-49 and male spouses $(n=10)$ on the study topic. IDIs were conducted among midwives $(n=3)$ working in the 3 health sub-districts operating in communities women participants resided. Table 5.1 provides a profile of socio-demographics of qualitative study participants. 
Table 5.1: Brief profile of qualitative demographics in study

\begin{tabular}{|l|l|l|}
\hline Data collection mode/demographics & Description/frequency & Total respondents \\
\hline $\begin{array}{l}\text { FGD with women (15-49 years) } \\
\text { in Bugri, Songo and Woriyanga } \\
\text { communities }\end{array}$ & $\begin{array}{l}10 \text { participants per community ses- } \\
\text { sion, three communities in total }\end{array}$ & 30 \\
\hline $\begin{array}{l}\text { FGD with men (18-59 years) in Wori- } \\
\text { yanga community }\end{array}$ & 10 participants in one session meeting \\
$\begin{array}{l}\text { IDI each with midwives(s) in } \\
\text { Bugri, Songo and Woriyanga health } \\
\text { facilities }\end{array}$ & 3 midwives in 3 health centers & 3 \\
\hline Gender & Female (30), male (10) & 40 \\
\hline Educational status & Educated (6), never educated (24) & \\
\hline Parity levels & Below 4 births (23), 4+ births (17) & Kusasi (27), Bimoba (7), Busanga (4), \\
\hline Marital status & Married (36), not married (4) & \\
\hline Ethnicity & other & \\
\hline
\end{tabular}

Three separate FGDs were held with women ( $\mathrm{n}=10$ at each community) aged 15-49 in three communities; Songo, Bugri and Woriyanga. Clinic staff at each community level assisted to select eligible women from functional mother-tomother support groups for women FGDs. Mother-to-mother support groups are informal women groups existing within the study settings that aim to provide education and sensitization of family planning and child caring practices to young mothers. One FGDs with male spouses $(n=10)$ was held in the Woriyanga community. Men were included in the FGDs to also obtain a fair idea of their influence in the use of family planning services by women at the household level. Woriyanga community was purposively selected for the men FGDs because of its heterogeneous nature, and provided a possibility to engage diverse men from different ethnic and social tribes for the FGD. Purposive sampling was applied to select 3 midwives for IDIs. Two midwives were selected from the Tempane zone while 1 midwife was from the Garu zone of the district. Midwives formed part of the study to provide health system and health provider insights of family planning services that were provided for women across the two districts.

FGDs guide for qualitative data collection for women were organized into three thematic areas; current and ever use experiences including decision making and contraceptive information received, support for contraceptive use and women prioritization of factors accounting for use and non-use of contraceptives (see Table 5.2). Each discussion group was comprised of young mothers with the 
following characteristics; young mothers in school or out of school, widowed or divorced, never married or married, current, and ever users of family planning.

Table 5.2: List of main questions included in the FGDs and IDI guide

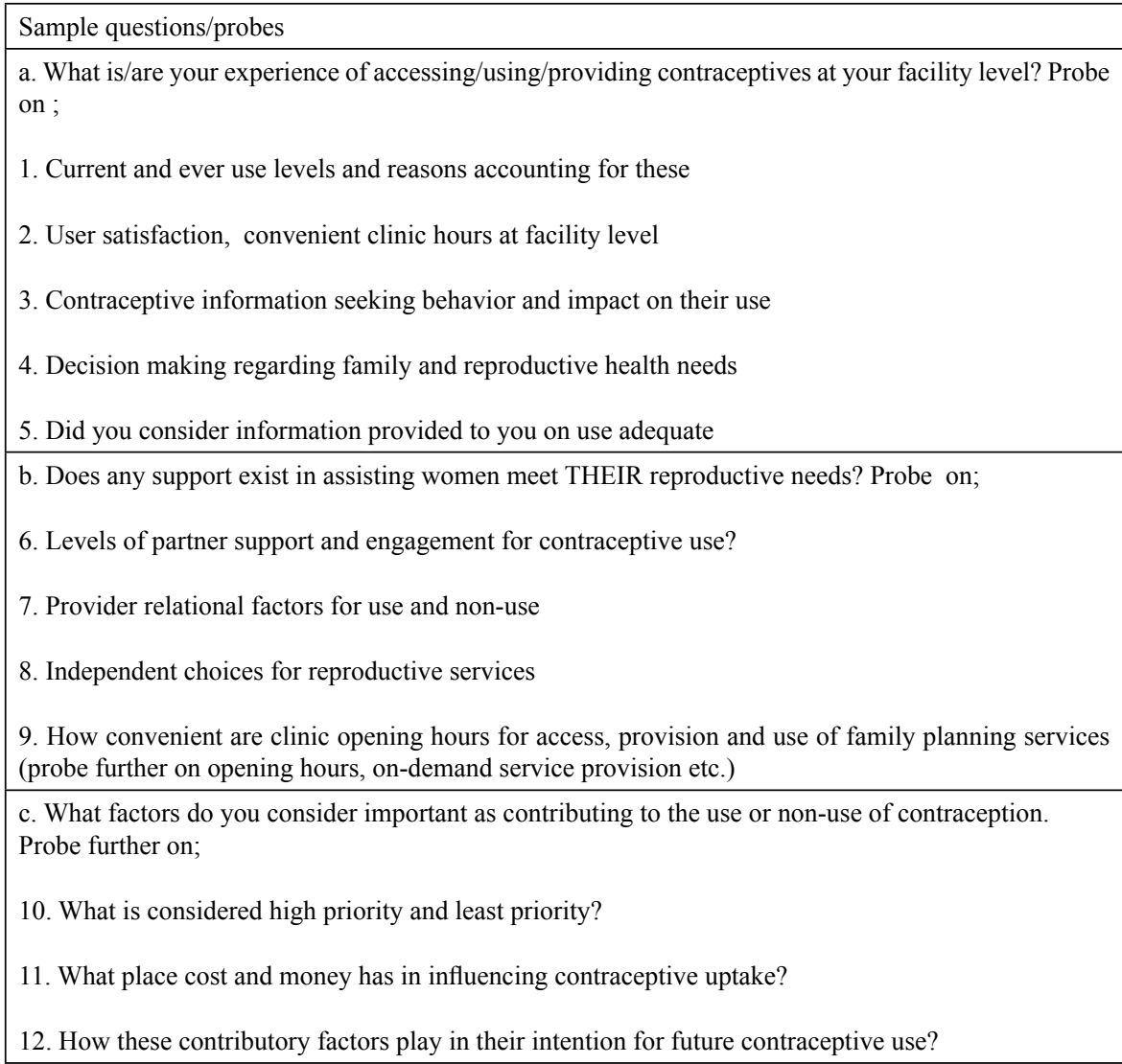

FGDs guide for male spouses elicited views on contraceptive awareness, practices and support for contraceptive use among both educated and noneducated male spouses. Each FGD (men and women) lasted an average of one hour and was conducted in Kusal language, predominantly spoken across the three communities. IDIs with midwives elicited their views on women's preferred contraceptive choices, social hindrances and institutional challenges confronting the delivery of family planning services. The midwives were selected from Songo, Woriyanga and Bugri health centers where women were earlier engaged for FGDs. IDIs took place at convenient clinic rooms that ensured less distraction and confrontation with patients visiting to seek care. IDIs were conducted in 
English. Midwives were included in the study to corroborate women and men views on service provision across the two districts. Qualitative data from all IDIs were audio taped. FGDs were transcribed verbatim from the local languages to English. IDIs were transcribed directly into English. All transcribed data were coded and analyzed manually based on three themes; contraceptive use, access on demand and future contraceptive use applying content analysis. Final emergent quotes are then presented in tabulated boxes. The qualitative results provide further clarity of expressions and views expressed in quotes.

Study approval was provided by the University for Development Studies, Tamale Ghana Institutional review board. The Regional Health Directorate and the District Health Management Team (DHMT) provided approval for health facility visits and follow-ups in all 24 visited facilities. Research assistants assisted all participants to fill out a signed/thump print consent form. Participants who were literate provided signed consent while non-educated participants provided thump print consent. In addition, all participants provided verbal consent during participant recruitment for the study.

\subsection{Results}

\subsubsection{Quantitative findings}

The results on socio-demographic characteristics of the survey respondents are presented in Table 5.3. The majority of respondents (473 representing 65.7\%) were within the age brackets of 26-28. Most respondents were never educated and more likely to be married. Parity levels were high for births between 1 and 4 with majority of women in monogamous family unions. 
Table 5.3: Background characteristics of survey respondents interviewed

\begin{tabular}{|l|c|}
\hline Socio-demographic variable & \\
\hline Age range & $56(7.8)$ \\
\hline $18-21$ & $191(26.5)$ \\
\hline $22-25$ & $473(65.7)$ \\
\hline $26-28$ & \\
\hline Languages & - \\
\hline Kusal, Bimob \& Bissa & $209(29.0)$ \\
\hline Educational status & $511(71.0)$ \\
\hline Ever Educated & $661(91.8)$ \\
\hline Never educated & $59(8.2)$ \\
\hline Marital status & \\
\hline Married & $460(63.9)$ \\
\hline Not married & $260(36.1)$ \\
\hline Family union of husband & \\
\hline Monogamy & $216(30.0)$ \\
\hline Polygamy & $504(70.0)$ \\
\hline Employment status & \\
\hline Unemployed & $679(94.3)$ \\
\hline Employed & $41(5.7)$ \\
\hline Parity levels & \\
\hline $1-4$ births & \\
\hline 4+ births & \\
\hline
\end{tabular}

Table 5.4 provides further analysis of the survey data using multinomial logistic regression. Women with male partner support, and those reporting satisfactory service use in the past where three times more likely to be current users of contraception. Women who received prior education and those with access to focused counselling on contraceptive use were twice likely to have ever used or be current users of contraceptives. Additionally, non-educated women were more likely to have access on demand with adequate prior counselling services. Current users with prior focused counselling had less intention to use contraceptives in the future. Socio-cultural norms and beliefs had a positive effect on young women who had ever used contraceptives. Outreach community services such as home visits by health staff were associated with the likelihood of contraceptive use for ever users. Client user satisfaction, and young women adequate knowledge of where to access services, was associated with ever use of contraception. Age had no effect on current and ever use. Age was also evidenced not to have any association with women access on demand and intentions for future use. 


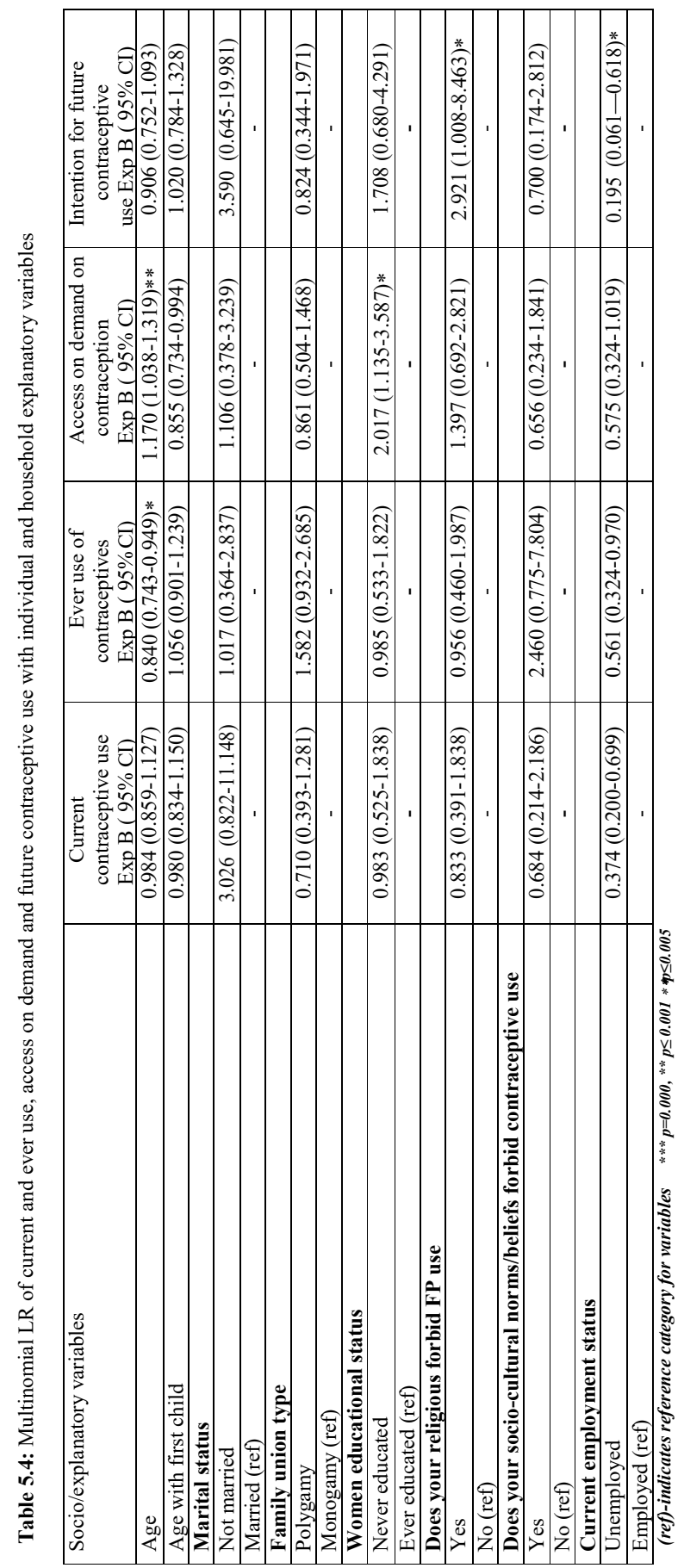




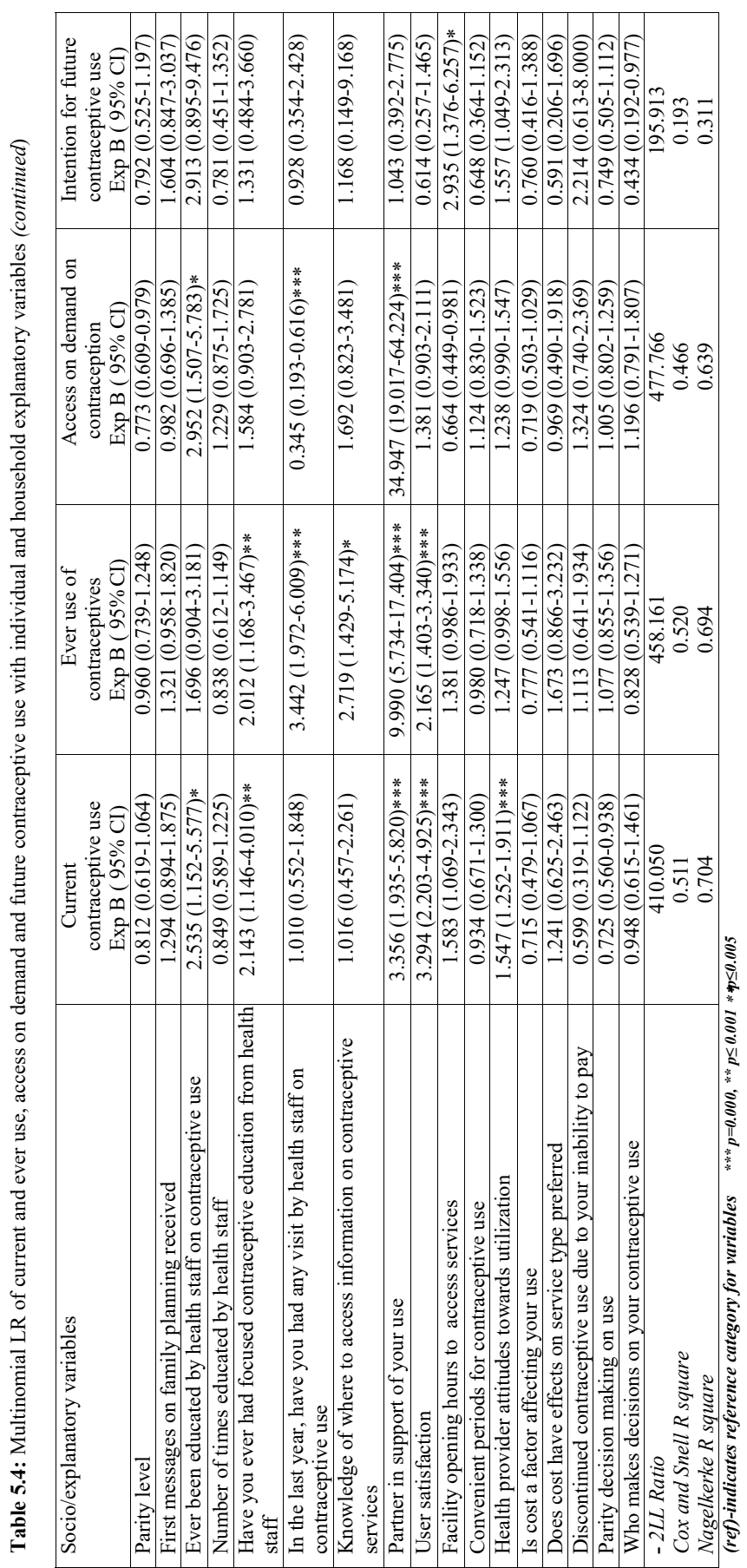


Non-married women were more likely to use contraception compared to married women. Dominant religious beliefs were positively associated with previous user's discontinuation of use over time (see Table 5.4). Having access on demand for contraceptive use is associated positively with a strong male partner support (OR=34.5, CI\% 19.01-64.22).

Women with male partner support where 34 times more likely to have access and support for continual use of contraceptives on demand. Female contraceptive awareness (ever been educated on contraceptive use) has a positive association with women who had access on demand for contraceptives. Women who received prior contraceptive education were twice more likely to have the intention to use contraceptives in the future compared to those with contradictory and misinformed information. Women's intention for future use is also significantly associated with the ability to pay for service provision, although current associated cost was not significant for current and ever users. Convenient opening hours at facility level has a positive association with future contraceptive use among young women. Young women in communities with a strong affirmation for their religion and customary belief systems indicated this could possibly affect their future contraceptive use intentions. Non-married young women were three times more likely to express their intention for future use of contraceptives.

\subsubsection{Qualitative findings}

\subsubsection{Ever and current contraceptive use}

During the FGDs, few young women expressed concerns about their previous or current use of contraceptives. Often cited issues includes; poor health staff relational behaviors, commodity supply shortages, women's own inability to stick promptly to stipulated dates, and weak partner support toward their desires for meeting their reproductive needs. Mothers reported they had often been scolded and treated poorly by health facility staff. Accounts of women threatened by health providers to withhold essential information and health care needs underscored some women expressions of healthcare consequences at facility levels. 
Box 5.1: Expressions of current and ever uses for contraception.

"I use to use family planning after the birth of my second child. After I gave birth, I have not been able to continue because of certain challenges. I cannot say because the nurses stopped coming here to educate and provide the services for us here again. The clinic is far and we cannot walk just..". (focus group discussant at Bugri)-quote 1

"The young nurses sometimes speak anyhow to us because they feel we have never been to school. They are not friendly at all and make me afraid to go there for services. I have been going to the Clinic in town because of their attitude towards us anytime we visit". (focus group participant-Bugri)-quote 2

"Is very bad to leave your work and come and sit in the queue for long before you are attended to. I don't like the way the nurses make us waste time anytime we go there". (focus group participant-Woriyanga)-quote 3

"As for me, I tried it when I was advised by a friend and I have never regretted. Even though I had few problems from the beginning, am still using it and its helping me plan my family". (focus discussant at Songo)-quote 4

Contraceptive use causes cancer, especially the pills because it affects your uterus causing you to develop abdominal pains all the time. My close friend cannot give birth because she was operated upon to rectify that problem caused by her use of family planning even though I initially advised her not to go for it". (focus discussant at Woriyanga)-quote 5

"We have found hidden ways to support women improve their sexual rights and opposition from their husband. We intend to support them plan and space their births which their husbands don't care about". (Midwife at health centre 2)-quote 6

"The men disturb us a lot about child bearing. We want to stay healthy and strong. In fact, ever since I started family planning, I really have peace of mind to concentrate on my business and take care of my six children". (focus group discussant with six children laments-Bugri)-quote 7

".........Most women are hiding from us and going to the clinics for it, how can we know they are not..... The birth of our children is threatened by this act. We will not sit down and agree to this". (39 year old man focus group discussant-Woriyanga)-quote 8

Quotes provide a summary of important views among women, men, and midwives on various themes discussed in separate FGD and IDI.

Other women recounted that most scolding and inhuman treatment were suffered from young nurses who took advantage of their low illiteracy levels to humiliate them. Users of contraceptive services who spoke in unanimity intimated the relief contraceptive use has brought them and their children. Men were often referred to as obstacles and were never seen by women to have enabled them to plan well for the next births. Most women did not hide their frustrations and their inability to meet their sexual needs through spacing or limiting. They indicated how their salient desires for contraception has been met through the 
covert use (see Box 5.1 quote 7). In separate FGDs with men to ascertain their roles in supporting their female spouses meet their reproductive needs, men appeared evenly divided on their support levels. While some indicated support for their wives towards contraceptive use, a majority spoke in strong opposition for women use for contraceptives. Opposition for use was based on some men opinions that contraceptive use was mainly to regulate their fertility levels and put "fear in them" as social heads of their families. Evident in the discussions were myths about how family planning was reducing their household labour force and a threat to enabling their wives become promiscuous. Some men expressed their views on this matter as espoused by one man's views captured in Box 5.1 quote 8 . Despite these, IDIs separately with midwives revealed most women desired these services, despite their opposition. Women therefore prefer to visit clinic settings at their convenient times and when privacy could be guaranteed to access services. Young women who had also received focused counselling services where most likely to be empowered to overcome opposition at home as well as address side effects as users. Midwives argued that contraceptive misinformation was high among women. This is largely due to low educational standards among women, tending to affect current and intentions among women for future use.

\subsubsection{Access on demand and future contraceptive use intentions}

Box 5.2 presents interactive key emergent responses across two thematic areas: access on demand and future use intentions. Women asserted some male spouses unwillingness to engage and support them to plan their families, which made it difficult for them not to disclose contraceptive use issues with their male spouses. Discussants however acknowledged that there are male spouses who support their wives to attend family planning clinics for contraception. 


\begin{tabular}{|c|c|c|c|}
\hline Theme & Young women (FGDs) & Men(FGDs) & Midwives(IDIs) \\
\hline $\begin{array}{l}\text { Access on } \\
\text { demand for } \\
\text { contraception }\end{array}$ & $\begin{array}{l}\text { Response/meaning: } \\
\text { "There was no peace at } \\
\text { home and now am about } \\
\text { four months pregnant, } \\
\text { even though I did not } \\
\text { intend to have it now" }{ }^{1} \\
\text { Signals unmet need for } \\
\text { spacing } \\
\text { "Some nurses are really } \\
\text { supportive. They keep our } \\
\text { cards against our } \\
\text { husbands notice..."w }{ }^{2} \\
\text { Supportive providers } \\
\text { enabling covert use by } \\
\text { women } \\
\text { "Contraceptive injection } \\
\text { can cause infertility. I } \\
\text { don't want to hear } \\
\text { anything on this... "w }{ }^{3} \\
\text { Wrong myths about } \\
\text { contraceptive use }\end{array}$ & $\begin{array}{l}\text { Response/meaning: } \\
\text { 'After I visited the } \\
\text { clinic together with my } \\
\text { wife and the nurse gave } \\
\text { us a talk on family } \\
\text { planning, my wife and I } \\
\text { choose the three month } \\
\text { contraceptive } \\
\text { injection....." m" } \mathrm{m}^{1} \\
\text { Willing men who } \\
\text { understand } \\
\text { contraception benefits } \\
\text { support their spouses } \\
\text { for use } \\
\text { "Health providers } \\
\text { assume with our wives } \\
\text { that we don't support } \\
\text { them...."m }{ }^{2} \\
\text { Men view arguments } \\
\text { against their non- } \\
\text { acceptance for use as } \\
\text { unrealistic and } \\
\text { inconclusive }\end{array}$ & $\begin{array}{l}\text { Response/meaning: } \\
\text { "For farming seasons, they } \\
\text { prefer services later in the } \\
\text { evening"mid" } \\
\text { Convenient hours preferred } \\
\text { for use is dictated by seasons } \\
\text { (rainy/dry season) } \\
\text { "Women don't sometimes } \\
\text { come consistently when they } \\
\text { are supposed to..."mid }{ }^{2} \\
\text { Women often forget about } \\
\text { return dates for next } \\
\text { injections. Reasons as not } \\
\text { properly counselled, high } \\
\text { illiteracy and low self- } \\
\text { empowerment accounts for } \\
\text { this }\end{array}$ \\
\hline $\begin{array}{l}\text { Future use } \\
\text { intentions }\end{array}$ & $\begin{array}{l}\text { Response/meaning: } \\
\text { "I cannot talk about future } \\
\text { use now because don't } \\
\text { know what will } \\
\text { happen...."w }{ }^{4} \\
\text { Uncertain future use of } \\
\text { contraceptives } \\
\text { "I am using now, but my } \\
\text { faith made me stop after I } \\
\text { was told by a friend. Not } \\
\text { sure is possible in } \\
\text { future"w" } \\
\text { Woman uncertain future } \\
\text { use may not be possible } \\
\text { because of her religion } \\
\text { "I may use in the future if } \\
\text { am widowed"w } \\
\text { Future use may be } \\
\text { prompted by } \\
\text { unavertable loss of male } \\
\text { spouse }\end{array}$ & $\begin{array}{l}\text { Response/meaning: } \\
\text { "For us to accept these } \\
\text { information's on } \\
\text { contraceptives use } \\
\text { continually, we need to } \\
\text { see how it will benefit } \\
\text { our lives" }{ }^{3} \\
\text { Men may assert to } \\
\text { accept contraceptive } \\
\text { use is they feel real } \\
\text { benefits associated } \\
\text { with it }\end{array}$ & $\begin{array}{l}\text { Response/meaning: } \\
\text { "Perceptions are changing } \\
\text { about the need to limit family } \\
\text { size....."mid" } \\
\text { People within communities } \\
\text { are beginning to think about } \\
\text { birth spacing and limiting in } \\
\text { response to current economic } \\
\text { situations affecting them } \\
\text { "having all the method choices } \\
\text { all the time will help build } \\
\text { trust and confidence for } \\
\text { use"mid" } \\
\text { Contraceptive commodities } \\
\text { availability allyear round can } \\
\text { provide trust for continual use } \\
\text { "Addressing those who have } \\
\text { experienced side effects helps } \\
\text { a lot in gaining trust among } \\
\text { current users"mid" } \\
\text { Clinic staff ability to address } \\
\text { health concerns arising helps } \\
\text { improve women trust and } \\
\text { disregard for myths on } \\
\text { contraception }\end{array}$ \\
\hline
\end{tabular}

FGD and IDI discussions under two themes. $\mathrm{W}^{\mathrm{n}}, \mathrm{m}^{\mathrm{n}}$, mid $^{\mathrm{n}}$ denote women, men, and midwives in the discussions. Superscript $\mathrm{n}$ denotes the number of view counts expressed by discussants.

Women could not provide definite responses when asked if they wish to continue contraceptive use (see Box 5.2, $\mathrm{w}^{4}$ ). They indicated that continuous 
contraceptive information and counselling by health staff, good relational issues with provider's, convenient clinic operational hours all year round, cost and opposition that do not threaten their marriages were weighted considerations for contraceptive use in the future. Women discussants identified other broader social related factors that affect their desire to benefit from contraceptive services. This included fear of side effects for current and ever users, lack of appropriate information for non-users and user financial constraints.

Though many women group discussants asserted that direct financial payments at clinic service points was not a challenge, associated cost including transportation for those with difficult access to the facility level was a problem. FGDs with women and men showed most women had unmet reproductive health desires (Box 5.2, w1). When discussants where asked a direct question on whether they wished to limit or space their childbearing, women were observed to be disadvantaged since they could not be definite about their responses. Women accentuated their access to choices for contraceptives on demand and its attendant health outcomes as a priority in dealing with their continuous contraceptive use. A larger proportion also indicated fear of later health outcomes and "misguided truths" from peers and older women as priority issues to address for their contraceptive non-use.

\subsection{Discussion}

Our findings highlight the importance of context-specific associations for women contraceptive use. While individual women's access to, and use of contraception may be influenced by broader social arrangements within the society, context clinic arrangements as exemplified by these results, show the need for user friendlier clinic hours for women contraceptive use. Contextual social and group norms such as male dominance, clinic operational hours, focused counselling/education could provide the basis for reaching women at various rural settings. Using a cross sectional survey in a heterogeneous cultural setting in rural Ghana, our study draws on the pluralism of women's unmet need gaps that need urgent attention if family planning programs want to succeed.

\subsubsection{Addressing women ever and current contraceptive use concerns}

Young women's satisfaction with service use is evidenced in this study to play a significant role among current users of contraception. The study shows that current contraceptive users may not have preconceived intentions for any future use of contraception. Thus, if current users needs remain unaddressed, future choices for contraceptive use may be illusive for reproductive program planning. This supports the evidence provided by the family planning literature in SSA that 
a wide gap exists between family planning intentions and use (DeRose, Dodoo, Ezeh, \& Owuor, 2004; Ross \& Hardee, 2013; Ross \& Winfrey, 2001). Contraceptive programs at the facility level must provide multiple choices, address accessibility and insecurity concerns to reassure and increase current user's satisfaction with contraceptive use.

FGDs evinced women would often start, continue, or have any future options for fertility regulation from their relational associations with health care providers. Some women in this study cited strict clinic rules prescribed by health providers for service use. Providers were blamed for often providing limited or "scanty" information that does not address individual women needs and concerns as well as often being shouted and humiliated at for those attending antenatal and post birthing services. Even among contraceptive users, women reiterated open censures suffered in the hands of providers when questioning on alternative choices for use. Views and expressions from this study personify the challenge that rural women face in meeting their reproductive intentions and reflect the consistency of evidence on provider relational challenges in rural Ghana and developing countries (Cleland et al., 2006; d'Ambruoso et al., 2005; Jewkes, Abrahams, \& Mvo, 1998; Yakong et al., 2010). To attain optimal results for improving provider relational issues, multi-dimensional context approaches that pay attention to culturally respectful and acceptable norms may reassure women meeting current and future options for contraception.

Another significant finding is that focused counselling and education prior to contraceptive use may contribute significantly among non-educated current users. Evidence of the positive infleunce of a woman's high educational attainment on her positive contraceptive use behavior in developing countries exist (Adebayo, Gayawan, Ujuju, \& Ankomah, 2013; Asekun-Olarinmoye et al., 2013; Do \& Kurimoto, 2012; Ononokpono et al., 2013). By responding to non-educated women concerns through focused counselling, contraceptive misconceptions, myths and cultural norms that divest women's intention for use could be addressed. This could potentially lead to community empowerment for women, with its attendant positive corollaries for health care seeking. This view supports various research findings that women economic empowerment could improve women reproductive health while improving women fertility life choices such as the incidence of domestic violence, freedom from prohibition and empowered decision making abilities (Corroon et al., 2014; Izugbara et al., 2010; Metcalfe, 2010; Rottach, Schuler, \& Hardee, 2009). This finding is important since the majority of women in Ghana live in rural areas, with high illiteracy rates. Focused counselling provides an alternative for many rural context health providers to reach underserved and un-educated populations. Integrated health services delivered in rural settings must ensure focused services whiles adhering to 
confidentiality for improved service utilization. This finding reemphasizes the call by family panning experts in 2012 to ensure that national efforts are accelerated and focused contraception is available for women in confined minority areas and among vulnerable population groups (Cleland \& Shah, 2013).

\subsubsection{Access on demand for contraception versus future use}

As suggested by our results, growing concern about dominant patriarchy roles in rural context influencing contraceptive use was significantly associated with women's ability to have access on demand for contraceptive use in this study. Thus, women who reported to have had access on demand significantly attained that with male partner support. This finding is significant and will require the re-examination of over-emphasized concepts that male partners total rejection of family planning in poor resource settings. The growing preference for small family sizes by women themselves could have pushed men to be assertive for young women concerns in this study, hence supporting them to meet their demands for contraception. These views are expressed strongly by other researchers as well. Specifically, male spouses may not necessarily be against birth spacing or limitation, which refutes the notion or perception about male roles in family fertility regulation (Cleland et al., 2006; Eliason et al., 2013). According to women in our study, strategies for scaling up contraceptive uptake with men inclusion must identify existing strengths for male support under the specific context to ensure that they are willing to continue to use contraceptive services. Cautiously, the drive and ambition within fertility debates for the inclusion of men for improving women reproductive rights must not sloppy efforts for context and system level changes in the broad scheme of providing care. Collective willingness by men, women and broader local context factors must be considered important at both the family and community level when using men as entry points for contraceptive acceptance and use.

Nevertheless, covert use as delineated among women discussants gives credence to the pervasive existence of unfair gender relations and poor spousal negotiation arrangements on fertility issues in rural Ghana. Covert use offered an opportunity for some women in the study to attain their demand for contraceptive use. For these women, continuous use is not a guarantee since they could be potentially abused if detected. Even among covert users, a convenient period that guarantees privacy is important. They assert in this study that their salient use must be "protected" by health providers, with convenient hours crucial for the continual use of contraception.

Adequate, prompt and timely behavioral education could support both educated and non-educated women attain the far reaching principle of the 
"calculus of conscious choice" beyond contraceptive use (Van de Walle, 1992). In rural Ghana, traditional norms influence a woman's life choices for child bearing. Myths and misconception of contraceptive use is still high (Parr, 2003) and could explain the basis for men's opposition to the use of contraception by their wives. Opportunities that assist women to adopt the current use regimes by improving their knowledge on failed method options will improve the consistency and correctness for contraceptive use. It is also proven that effective counselling could assist women to make postpartum contraceptive choices later in life (Eliason et al., 2013). Clinic counselling services must incorporate willing men who support contraceptive use as role models for community reproductive health services that target improving male support for women use for contraception. Steps by health facility staff to ensure that women who desire privacy and confidentiality meet these desires as covert use could still be practiced by women within settings that men tend to support joint spousal contraceptive decision making.

\subsection{Policy Implications}

Providing client centred, culturally sensitive and acceptable care that respects women's preferred convenient clinic hours for service use has the propensity to improve user satisfaction and subsequent future use shown from this study. For current user's preference for covert use, services should be delivered confidentially. Clinic settings must design structures that guarantees continued confidentiality and provide mechanisms that address individual health concerns during use. Additionally, policy decisions for male targeting to improve contraceptive use by health program planners must first identify context preference by women for their inclusion or exclusion. At local community level, health advocacy and campaigns must address context poor health seeking al reasons that perpetuates myths and misinformed clients of safe and beneficial clinic services. Broader engagements using existing local clinic health committees in the design and implementation of contraceptive user interventions could contribute to wider acceptance by men and larger family ties for women in rural settings. At a national level, the wider incorporations of socio-cultural and social anthropological inclusions into nursing training curricula will improve health care provider's worldviews of the role of cultural context factors for healthcare delivery among diverse social groups.

Though issues of bias could be raised on the age restriction for the quantitative part for the study, it does not affect the validity of findings. Age was not also found to have an association with contraceptive use in this study. Married women or those in a union are often the standard reference group for reproductive health intervention and formed majority among our study age group. This age target has 
enabled the study to examine specially issues around young women, besides the other views from women of reproductive age and men during FGDs and IDIs. We acknowledge that the heterogeneous nature of communities could possibly have influenced participants to hide critical information on study outcomes from research assistants who were recruited from the district. Data quality was guaranteed by ensuring research assistants were well trained to conduct interviews with cultural sensitivity as well as minimize recall bias. Methodologically, our choice of study design limits our ability to draw strong inferences with contraceptive use. The relatively large sample and district wide coverage of the study provides a good basis for a wider acceptance of results and its greater use and generalization in similar context across SSA.

\subsection{Conclusion}

This study exhumed rural context associations influencing young women reproductive health needs in rural Ghana. As indicated by this study, to improve access to contraception demand and use, convenient clinic hours desirable by women and attentive health staff attitude must be reached, especially in areas where covert use and family work arrangements may permit women only on certain periods. Health providers need to incorporate focused counselling and educational support, guarantee privacy and support the existence of male partners willing to support women meet their fertility desires. To establish how covert users could be better protected and supported for improved contraceptive uptake in any rural setting, research is needed for ascertaining prevalence of covert users and how existing community systems can support intended users. 



\section{CHAPTER 6}

Stakeholder's experiences, expectations and decision making on reproductive care: An ethnographic study of three districts in northern

Ghana

Draws upon work under review:

Ayanore, MA., Pavlova, M., Biesma, R., \& Groot Wim. Stakeholder's experiences, expectations and decision making on reproductive care: An ethnographic study of three districts in northern Ghana 


\section{Abstract}

Introduction: To engage stakeholders in the provision of reproductive care, it is necessary to study the varied stakeholder views on reproductive care services.

Methods: An ethnographic study approach was used where field data was collected between March-May 2015 in three rural districts of northern Ghana. Data were collected through in-depth interviews and focus group discussions among women with recent birth experiences $(n=90)$, health care providers $(n=16)$ and policy actors $(n=6)$. Each stakeholder participant's audio file was transcribed, and repeatedly read to identify similar and divergent views in the data. A coding scheme guided the coding processes. All transcripts were then imported into QSR NVivo 11 for further analysis.

Results: Four themes emerged: the impact of gender roles on reproductive care needs, experiences with meeting reproductive care needs, expectations about reproductive care needs, and the policy setting and decision making processes on reproductive care needs. Women accentuated that sex and sexuality values of men have changed over time, and drives gender roles, parity levels and decision making on reproductive care needs at community levels. Sexual stigma on reproductive care reduces the willingness of women to voice poor experiences related to their previous reproductive experiences. Stakeholder's highlighted that clinical treatments for post-abortion care are minimally covered under the fee exemption policy for antenatal and postnatal care. Policy processes on service delivery protocols still is top-down in Ghana.

Conclusions: More needs to be done to improve reproductive health care needs of women in Ghana. Health teams could contribute to that. Private sector participation and informal community supports are encouraged to advance these reproductive care services. 


\subsection{Introduction}

Sexual reproductive health of women is influenced by multiple individual and societal needs in SSA (OlaOlorun et al., 2016). The double burden of early sexual debut and marriage within the SSA region exacerbates the need for timely sexual and reproductive health care (DeJong, Shepard, Roudi-Fahimi, \& Ashford, 2007; Zabin \& Kiragu, 1998). This is further enhanced by the rising unmet reproductive health care needs among women in the region (see Chapter 1 and 2 ). Aside the health consequences of unmet reproductive health care needs, the economic burden of reproductive health is huge in most developing countries (Vlassoff, Walker, Shearer, Newlands, \& Singh, 2009). This economic burden coupled with the adverse effects of reproductive health near-misses, aggravates long term reproductive care needs of women in later life. Global estimates show that $\$ 68$ million to $\$ 76$ million annually is needed to manage near-misses of unsafe abortion at facility level following unattained reproductive services needs in developing countries (Vlassoff et al., 2009). A recent review of unmet sexual and reproductive health needs in West African show that unmet reproductive needs are still high in some countries (as discussed in Chapter 2)

In Ghana, priority-setting for reproductive health service interventions is known to be rudimentary with little wider stakeholder involvement (Mayhew \& Adjei, 2004; Seddoh \& Akor, 2012). Recent evidence in Ghana shows progress on family planning access, knowledge and awareness for reproductive health care (GSS et al., 2014). The recent Demographic Health Survey finds that sexual freedom of women aged 25-49 has increased (GSS et al., 2014). Nonetheless, sexually active adolescents in Ghana are known to use contraceptives inconsistently, with lower reported levels of contraceptive use, $25 \%$ and $40 \%$ among girls and boys aged 15 19 respectively (Doyle, Mavedzenge, Plummer, \& Ross, 2012; van der Geugten, Dijkstra, van Meijel, den Uyl, \& de Vries, 2015). Fertility preferences and intentions on the timing of child bearing and the desired number of children appear to drive reproductive decisions (Miller \& Pasta, 1995). Community level evidence on reproductive services uptake such as family planning using the "Zurugelu Approach" in Ghana shows that introducing and sustaining reproductive change requires attention to the needs and concerns of not only women (Dalaba et al., 2016). In particular, the involvement of the male spouse and the hospital staff effectively promotes family planning and counselling services (Dalaba et al., 2016). But how exactly is the Ghanaian health system responding to the changing reproductive needs of women through information and counselling services? Health sector reports in Ghana advocate improving women reproductive autonomy to increase reproductive health service utilization (GHS, 2009; $\mathrm{MOH}$, 2008). More so, evidence of social conditions impacting reproductive care across 
stakeholder groups enhances user utilization and satisfaction. Most studies in Ghana relates to social, econmic and socio-gendered role effects for reproductive care in rural Ghana. Knowledge on how health care provider's experiences shape and influence women preferences and autonomy on reproductive care services use is limited in Ghana despite the growing consensus for broad stakeholder involvements in the provision and use of reproductive care services.

In recognizing the need for broad stakeholder engagement to advance reproductive care provision and utilization, it is necessary to jointly study the varied stakeholder views on reproductive care services. Specifically, there has been a growing need to understand the shifting expectations of user preferences on reproductive care services (Halley Crissman et al., 2013). Priorities in policies on reproductive health are described in one study as ad hoc and limited (Mayhew \& Adjei, 2004). This situation exists despite the increasing calls for public-private partnerships in the delivery of services. In addition, the question can be raised whether policy processes address user preferences for comprehensive, reliable and user-friendly services at the facility level as outlined in Ghana's reproductive health policy (GOG, 2000). In what directions do women perceive gender roles to influence their reproductive decision making and choices for care?

To identify these gaps, views of users of reproductive health services (women), health staff and policymakers were analyzed concurrently in three rural districts of northern Ghana. At the health policy level, this study provides stakeholder opinions of how women reproductive care shape reproductive behavior, choices, decision making, and priority-setting for policy decision making in rural Ghana. Our results add to the current knowledge on how various stakeholders' roles, decision making and priorities impact reproductive health services provision and use in Ghana.

\subsection{Methods}

\subsubsection{Study design and settings}

This study adopted the same design approach outlined in Chapter 4. Data were collected simultaneously within the same period for Chapter 4 and Chapter 6 of this dissertation. The design adopted ensured that all stakeholder concerns surrounding the study aim were given a "voice"(Allen, 2011). It provided the opportunity to examine the complexity of reproductive care needs and how the interactions of women, health staff and policymakers influence the provision and utilization of reproductive care services. The three districts examined in Chapter 4 were assessed on this study aim (see Chapter 4 for districts). As justified, 
the selected districts have poor indices of maternal and reproductive health outcomes among a total of 13 districts in the study region. Proximity and resource availability for data collection also influenced the choice of study districts. In each of these districts, three remote facilities providing reproductive health services in each district were selected (9 facilities in total). We also included Bolgatanga, the capital of the Upper East region as one of the settings because some policy interviewees resided in this area.

\subsubsection{Data collection and sampling procedures}

Data were collected among three groups of stakeholders: women with records of recent births (2 years prior to study), health staff, and policymakers. FGDs were conducted with women and health staff while IDIs were held with policymakers. To select women participants, midwives in the included facilities provided information on 15 women per facility with recent birth experiences using data from antenatal and postnatal facility registers. In total 135 women were selected as potential participants in all 3 districts. At each district level, community fora meetings were held across all 9 facility settings to select women participants for the study. An inclusion checklist with the following criteria was used to rank final participants at each facility: women should be physically present at meetings, consent to participate, interested in the study and able to share prior knowledge related to the study aim. At each facility meeting, 10 participants with high rating on these creteria were selected. A convenient sample of 30 eligible women per district took part in the FGDs (3 FGDs per district with 10 women in each FGD). Thus, in total 90 women took part in the study. Across the three facilities per district, health staff in one of the facilities was selected. In these district facilities, one midwife, a senior staff in-charge of facility, and facility nurses (on average 3 to 7 persons per district) responsible for reproductive health services were recruited as district health staff participants. Policymakers were later recruited at both district and regional level. They included 2 private policy program implementers and 4 public policymakers. FGDs with women were conducted in one main district local language; Talensi (Tali), Nabdam (Nabit) and Bongo (Grune). All IDIs were conducted in English. FGDs and IDIs were conducted using sample structured guides (see Box 6.1). Approval for this study was provided by the Institutional Review Board of the Navrongo Health research Centre, Ghana. 
Box 6.1: Sample question used to assess all stakeholder groups

\section{Reproductive health care experiences, expectations and policy process on reproductive health care} needs

a. What experiences, expectations do you have with regards to using/providing_reproductive health care? Probe further on social, gender and environmental drivers for women independent life choices in reproductive health care.

b. What experiences underpin reproductive preferences/needs among users and providers in the facility/districts level? Probe further on challenges, barriers that affect your ability to deliver basic and comprehensive reproductive care services at the facility/district level?

c. What expectations define the reproductive preferences in provision (health staff, policymakers) and its use (women)? Probe on how each stakeholder meets and unmet expectations.

d. What priority setting informs reproductive health choices and preferences in the facility/ district? Probe on concerns policy decision making process have for users in rural settings. Probe further on policy standards in use and what they seek to attain in reproductive rights issues

e. How does the policy processes address user preferences for reliable and user-friendly services for women, health staff and policy makers. Probe further on services delivery mode, previous experiences impact on current reproductive needs? Follow up with probes on reproductive policy implementation processes and inclusiveness and how this relates to meeting user reproductive care preferences.

\subsubsection{Data analysis}

In conducting the interviews, the ethnographic approach allowed for continuous inductions and verification of all stakeholder views. FGD and IDI audio files were transcribed verbatim into English by two research team members (the principle researcher and an assistant). We used two research team members to minimize single level biases in our analysis. Transcripts from all stakeholder groups were repeatedly read by the principle researcher and the assistant to identify similar and divergent views in data. All individual stakeholder transcripts were finally checked for accuracy and consistency with the original audio files. The research team then developed a coding scheme to guide the coding for each stakeholder group response based on the aim of the study. To ensure consistency among the two coders, we developed coding rules to facilitate the process. An initial coding for transcripts was undertaken by the principle researcher and the assistant using the principles for open coding (Charmaz, 2006). All transcripts were then imported into the computer-assisted qualitative data analysis software QSR NVivo 11. In NVivo, one research team member undertook a further induction (open coding) for stakeholder concepts while another team member 
audited the first coder work to ensure consistency and reliability of coding in NVivo. Each imported transcript was analyzed in NVivo separately. Constant comparison enabled a further reduction of concepts, codes and nodes into themes (Glaser, 1965). To show how individual stakeholder views relate to each other and our study aim, we used the model explorer tool to map out how each stakeholder themes relate to each other (Welsh, 2002). All codes were further refined until we reached saturation. To guarantee the reliability for our results, we estimated interrater coding reliability for all coded data. Women, health staff and policymakers recorded $\mathrm{r}=0.98, \mathrm{r}=0.96$ and $\mathrm{r}=0.94$ respectively. Mapped out results for all stakeholder themes are tabulated and further illustrated in the results section.

\subsection{Results}

\subsubsection{Characteristics of participants}

Parity levels of the 90 women in the FGD ranged between 1 and 7 births. Only 13 women ever had a home birth. Four women reported pregnancy miscarriages. Six women reported they ever sought post abortion care. Two women indicated they had to pay for post-abortion care services. Ever use and discontinuation of contraceptive use was reported by 18 women. Few women however knew where to obtain related services such as sexual transmitted infection (STIs) information and treatment. No woman reported to have been examined for any related reproductive cancers. The health staff was represented in the study by 3 midwives, 3 senior health nurses and 10 junior community/enrolled nurses with varying levels of working experience within the Ghana Health Service. Policymaker's participants were responsible for policy dissemination at facility, community, district and regional level.

\subsubsection{Findings}

Four main themes emerged from the qualitative analysis of the transcripts: 1) the impact of gender roles on reproductive care needs, 2) the experiences on meeting reproductive care needs, 3) the expectations about reproductive care needs, and 4) the policy setting and decision making processes on reproductive care needs.

\section{The impact of gender roles on reproductive care}

The gender role theme emerged among women only. Table 6.1 presents women's opinions of how gender dynamics drive their reproductive needs from early 
adolescence to later in life. Women indicated that they trusted that external friendship ties could assist them to meet their reproductive choice need, relative to family support. At the community level, the majority of women accentuated that sex and sexuality values of men have changed over time. Specifically, women asserted that male spouses often accept their need for reproductive care if they contribute to the economic roles at the family level.

Women admitted that male views on women sexual passivity may influence male demands to control their sexual pleasures. They avowed that these beliefs drive the gender roles, parity levels and decision making on reproductive care needs. Social perception and attitudes on decision making favours male spouses more than women. Thus, women indicated that their ability to economically support male spouses to meet family care needs, gives them leverage to covertly meet certain reproductive care needs (Table $6.1 \mathrm{w}^{3}$ ). Women opined their resolve to covertly use certain reproductive health services at the facility level if the spouse does not recognize their reproductive needs (Table $6.1 \mathrm{w}^{5}$ ). Most women favoured reproductive services that address their psychological needs rather than just rendering products and services. Most women accentuated that services addressed at their emotional and physical self-worth were absent in their facility. In the absence of psychological support, strong family opposition to women making independent reproductive choices have an impact on women preferences. Women indicated that negotiating in case of familial opposition is often difficult (see Table 6.1, $\mathrm{w}^{6}$ ).

Gender roles in this study also showed women expression of health staff cluttered controls over women at health facility centres. Some women indicated that they had been skipped or ignored by the staff, or received insufficient attention by nurses during post-partum family planning care. This often happened if health staff perceived that women challenged the status-quo when receiving reproductive services as asserted by women.

Some women also asserted they would often be skipped to be attended to during antenatal visits if they try to question female nurses of unmet care demands at the health facility as expressed in Table $6.1, \mathrm{w}^{10}$. When male health staff power relations were quizzed, women indicated that the impact of male nurse staff on their reproductive was limited. The majority of women however indicated that male community volunteers assisted and provided them feedback on addressing reproductive needs (Table $6.1, \mathrm{w}^{11}$ ). 
Table 6.1: Women views on gender role impacts on attaining their reproductive care needs.

\begin{tabular}{|c|c|}
\hline Theme 1 & Women accounts \\
\hline $\begin{array}{l}\text { Gendered } \\
\text { role } \\
\text { impacts on } \\
\text { reproduc- } \\
\text { tive care }\end{array}$ & 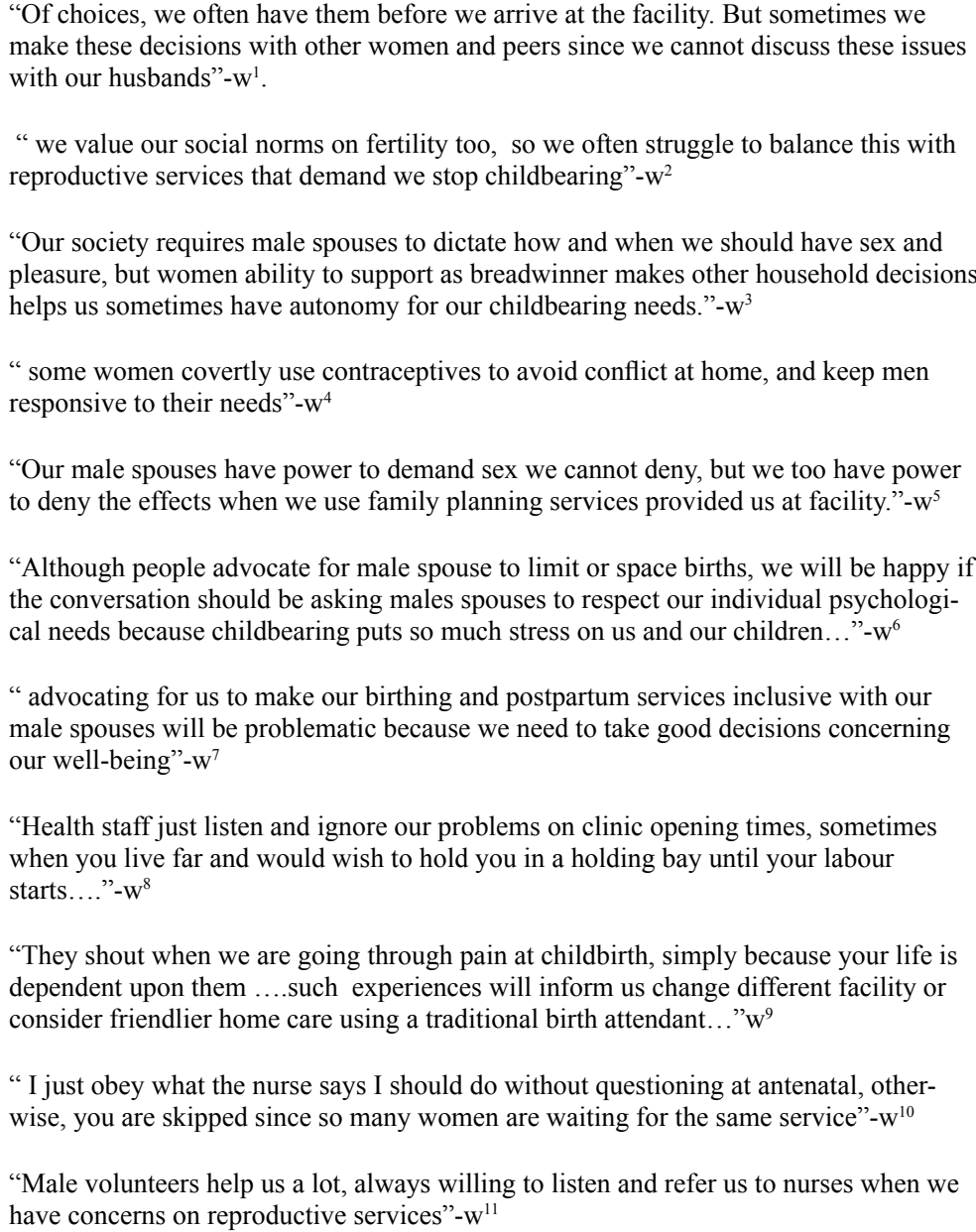 \\
\hline
\end{tabular}

$\mathrm{W}^{\mathrm{n}}$-W represents women views and superscript indicates number of record presented in Table

\section{Experiences relating to reproductive care needs}

The experiences-related theme was observed among all three stakeholder groups (see Table 6.2). User experience enumerated here relates to the lack of reproductive services non-differentiation among users, the cost implications for post-abortion care, and the social stigma associated with demanding abortion care services. Women asserted that the health staff did not differentiate between adolescents and women with higher parity desires (Table 6.2, $\mathrm{w}^{1,6}$ ). 
Women reported a lack of facility space for services related to infertility counselling, and STI diagnosis for women. Health staff indicated that referrals to district or regional facility would occur in instances when users feel traumatized by social stigma (Table $6.2, \mathrm{hs}^{2}$ ).

Women $(\mathrm{n}=10)$ who experienced pregnancy terminations (induced abortion or miscarriage) payed high fees to obtain post-abortion care, which was never a rewarding experience. A woman recounted paying USD 25.00 to obtain postabortion care services (Table 6.2, $\mathrm{w}^{2}$ ).

Policymakers indicated that clinical treatments for post-abortion care were minimally covered under the fee exemption policy for antenatal and postnatal care (Table $6.2, \mathrm{p}^{2}$ ). Poor communication by nurses was observed to contribute to the unwillingness of women to use contraception (Table 6.2, $\mathrm{w}^{3}$ ). Social stigma contributed to negative experiences with reproductive demands at facility and community level. Few women reported that the health staff's unaccommodating attitudes towards young women and adolescents seeking abortion care lowered the trust among new users. Specifically, women mentioned they have been traumatized by bad experiences when they accompanied their young adolescent girls for antenatal care (Table 6.2, $\mathrm{w}^{5}$ ). The majority of health staff provided only limited family planning products. This was seen by women as a deterrent for visits to community health facilities for reproductive services (Table 6.2, hs $\mathrm{s}^{1,4}$ ). Health staff stated that their inability to deliver multiple reproductive services is because most clinical reproductive services are not budgeted and supported locally.

In instances where health staff cannot meet women expected reproductive service preferences, users and local actors are often unable to explain what services are better met at community facility centres (Table 6.2, hs $\mathrm{s}^{5}$ ). Policymakers specified that health facility level evaluations of staff performance are one way to monitor reproductive and other general services delivery.

Policymakers recognized that new health infrastructures prioritize user needs, such as adolescent reproductive friendly centres spaces to cater for individualized needs in new public facilities. The need for privacy to reduce the negative stigma among intended users, particularly counselling for HIV/AIDS services has improved (Table 6.2, $\mathrm{p}^{3}$ ). Stigmatization may sometimes reduce the willingness of women to voice poor experiences related to their previous reproductive experiences. Policymakers reported periodic community engagement to understand user's preparedness and demands for quality and satisfactory service needs. 
Table 6.2: Stakeholder experiences with service provision to meet user reproductive preferences

\begin{tabular}{|c|c|c|c|}
\hline Theme 3 & Women & Health Staff & Policymakers \\
\hline $\begin{array}{l}\text { Expecta- } \\
\text { tions on } \\
\text { reproduc- } \\
\text { tive care } \\
\text { needs }\end{array}$ & 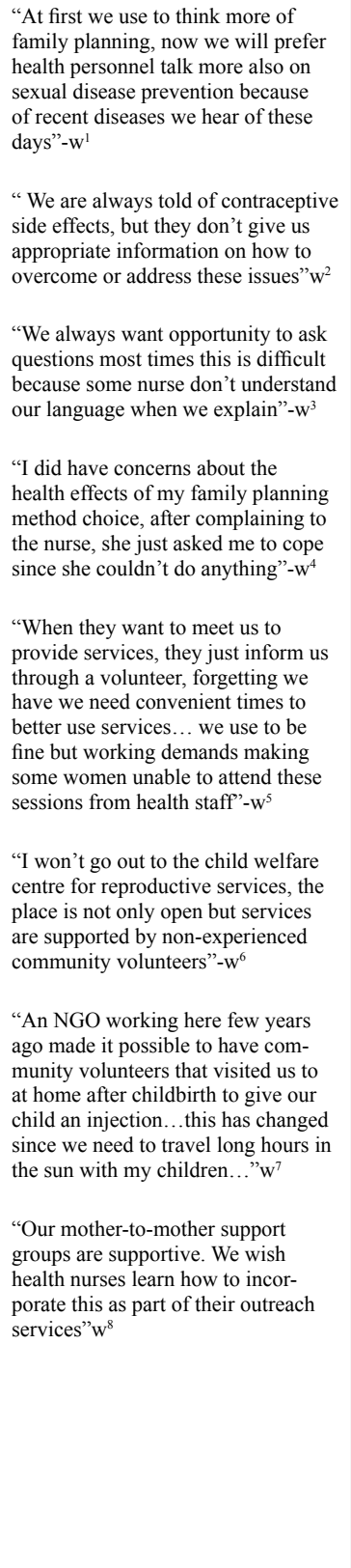 & $\begin{array}{l}\text { "some stuff do not know all the } \\
\text { components of the Cairo targets } \\
\text { on reproductive health...they are } \\
\text { basically involved in family planning } \\
\text { and few components of antenatal and } \\
\text { postnatal care"-hs' } \\
\text { "even when we know what women } \\
\text { need, can we meet that simply by } \\
\text { knowing, more has to be done from } \\
\text { the Ministry of health to support } \\
\text { in early diagnosis and treatment } \\
\text { such as opportunistic infections on } \\
\text { STIs, reproductive cancers, and safe } \\
\text { abortion care"-hs" } \\
\\
\text { "I did not know we are expected to } \\
\text { provide infertility services, I always } \\
\text { thought it was the role of the private } \\
\text { sector in such services"-hs }{ }^{3} \\
\text { "Sometimes we have difficulty } \\
\text { dealing with women own difficulties } \\
\text { when we don't know the appropriate } \\
\text { health counselling demands to meet } \\
\text { their need"-hs }\end{array}$ & $\begin{array}{l}\text { "Improving staff skills is something } \\
\text { the ministry and at our level we con- } \\
\text { tinue to undertake. We acknowledge } \\
\text { current inabilities to provide services } \\
\text { such as abortion care, post-abortion, } \\
\text { and fertility treatments mostly in the } \\
\text { public sectors"-p" } \\
\text { "What we need to do more in } \\
\text { meeting user expectations is to invest } \\
\text { more in infrastructure. At our policy } \\
\text { level we acknowledge the difficulties } \\
\text { although we cannot drive this big } \\
\text { policy push"-p" } \\
\text { "The health centers or district } \\
\text { hospitals although some staff have } \\
\text { received training cannot even } \\
\text { provide safe abortion services, this } \\
\text { sector has entirely been taken over } \\
\text { by the private sector where limited } \\
\text { people may access services because } \\
\text { of cost and stigma"-p" } \\
\text { "Helping women manage health } \\
\text { consequences is a key component } \\
\text { of our counselling services. One of } \\
\text { the greatest challenge is addressing } \\
\text { meeting individualized focused } \\
\text { preference needs where health staff } \\
\text { are limited"-p" } \\
\text { structures and groups at community } \\
\text { level more involving, accounTable } \\
\text { and supportive to address users } \\
\text { expectation at our facilities"-p" }\end{array}$ \\
\hline
\end{tabular}




\section{Expectations on reproductive care needs}

The expectations-related theme also emerged among all three stakeholder groups. Women's views here reflect assertions that the health system has overly emphasized and relied on products and services to meet their reproductive health needs. Examples of how facility level structures meet or fail to meet women's needs are reported by health staff. At the policy level, what needs to be done more to meet user needs was highlighted. Women also raised the need for more information in meeting health effect concerns, particularly for contraceptive services. The majority of women indicated they are hardly provided with adequate and appropriate information on how to overcome negative side effects. Services were more focused on family planning with little attention paid to sexual disease prevention, opportunistic infections such as reproductive cancers and infertility treatments (Table 6.3, $\mathrm{w}^{2} \&{ }^{3}$ ). Health staff affirmed they may not be able to meet some reproductive care needs when they lack appropriate diagnostic and treatment tools. Policymaker's views substantiated the health staff perspectives, emphasizing services such as shortcomings in abortion care, infertility treatment, and physical infrastructure deficits, which have to change dramatically to meet user preferences (Table 6.3, $\mathrm{p}^{1 \& 2}$ ).

Even when reproductive health services provided are desired by a user, the inability to ask questions and be provided feedback due to language barriers limit meeting desired contraceptive needs. A women recount a health staff rhetorically saying "I couldn't do anything". This was agreed by many women as a common response when they push further on meeting certain expected services at the facility level (Table 6.3, $\mathrm{w}^{4}$ ). Additionally, women wanted more integrated services rather than visiting severally separately. The lack of knowledge about women's basic infertility needs by health staff and the lack of facility level infrastructure add to the inability to meet integrated reproductive care needs for women. Staff accounts showed that some women rely on the few available private medical centres to meet such basic service needs. Some health staff agreed partly to these women expressions of unmet reproductive expectations. This was driven by health staff assertion that they lacked appropriate counselling skills beyond family planning (Table $6.3, \mathrm{hs}^{1}$ ). Policy makers cited individualized counselling as a great challenge to health staff, despite improvements in skills training on the job for health staff (Table 6.3, hs ${ }^{4}$ ).

While women view timely information sharing by health staff to influence their reproductive care choices, health staff inabilities to address their psychological needs creates low self-esteem for intended users. This impacts women's abilities to realize their desired fertility choices. Health staff avowed that unmet women reproductive psychological needs are minimally met because psychologists perceive reproductive care not as a priority (Table 6.3, hs ${ }^{5 \& 6}$ ). Health staff and 
policymakers affirmed that the contextualization of most reproductive health targets would improve expectations of psychological support for reproductive care (Table 6.3, $\mathrm{hs}^{7}$ and $\mathrm{p}^{6}$ ). Women also alluded to expectations of experiencing services delivered to them at closer proximities and at their home.

Women who indicated that their spouse supported them in taking reproductive decisions, wish to see services provided at home, in comparison to child care services currently provided by some non-governmental organization working in some of the districts in our study. Women supported the need for the formal recognition and adoption of informal social support groups such as mother-tomother support groups to provide them formal health advocacy information and psychological support. Policy makers recognized the growing concerns about informal social support, agreeing that the health system should be more accountable and socially supportive to meet women reproductive needs (Table $\left.6.3, \mathrm{p}^{8}\right)$. Facility level reproductive services have been tailored towards increasing patient-health staff contact hours, so as to address more user complaints and unfriendly relations often reported by women (Table 6.3, $\mathrm{p}^{5}$ ). 


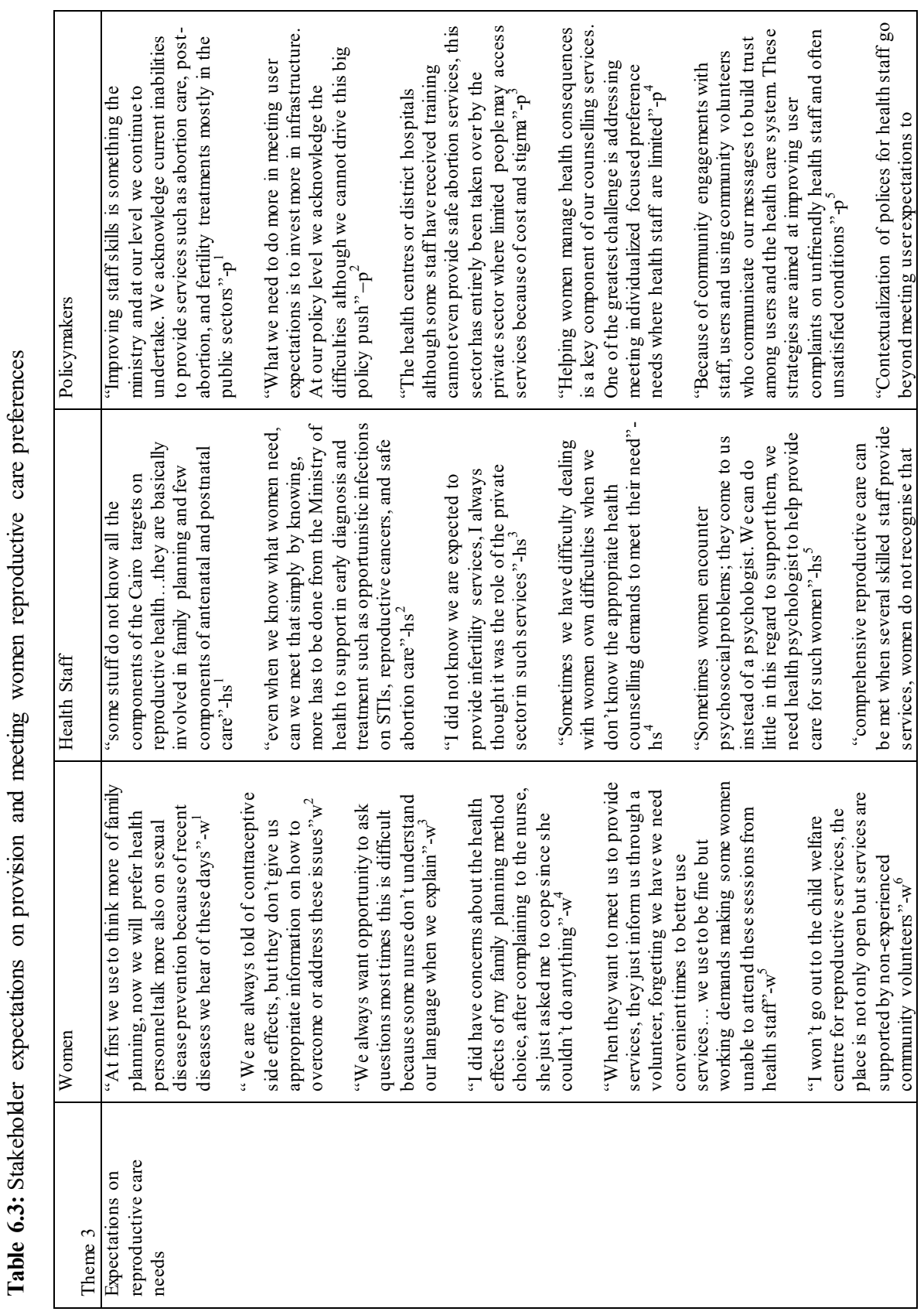




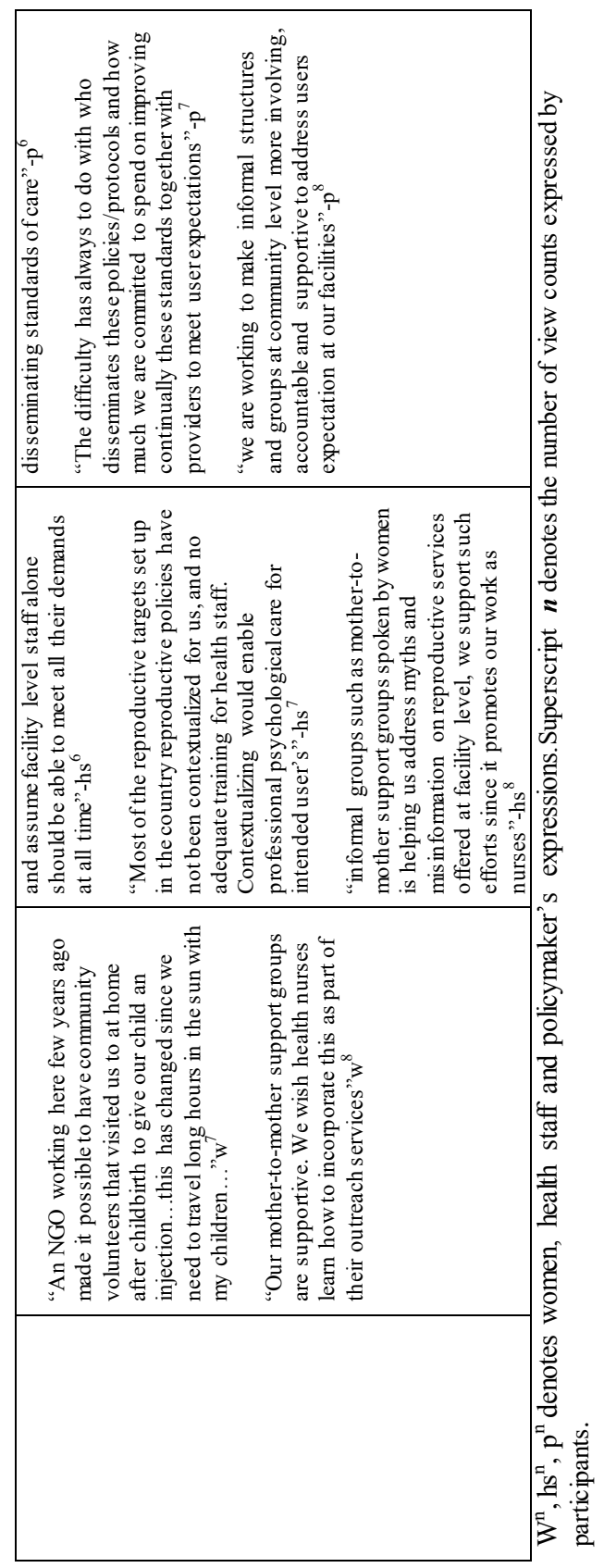




\section{Policy setting and decision making on reproductive care}

All stakeholder groups' raised the theme of policy and decision making. Policymakers openly agreed that most reproductive care services are driven by donor funding, although this funding has dwindled over the years. Hence most decision making priorities align to who provides funding and to which areas funders allocate their funds (Table 6. ${ }^{4}, \mathrm{p}^{1}$ ). Policy implementers and program managers only have the opportunity to prioritize a few activities from the reimbursements received from the national health insurance authority as reported by public policymakers (Table 6.4, $\mathrm{p}^{4}$ ). Most activities prioritized at this level are for reproductive advocacy (Table 6.4, $\mathrm{p}^{5}$ ). A health care worker recalled that refresher trainings are the only periods they are introduced to reproductive service or products. The policy processes only involve health staff when they have to disseminate skills for service improvements (Table 6.4, $\mathrm{p}^{6}$ ).

Most women in the two districts acknowledged that they have been asked to participate in health program manager's advocacy meetings in the past, but few policy changes have occurred. They indicated that even though they provide suggestions of how their facilities could better address their reproductive needs, they have become tired of these policy-provider engagements since their inputs are minimally incorporated into the services delivery (Table 6.4, $\mathrm{w}^{1,2},{ }^{4}$ ). Most opinions from health staff blamed policy implementers at the district level for doing little to improve women reproductive care needs (Table 6.4, hs ${ }^{5}$ ).

At the socio-political level, political commitment has driven major reproductive care needs over the last decade according to public policymakers (Table 6.4, p6). Some policymakers recalled how the Sector Wide Approaches (SWAp) and the MDGs targets have made contraceptive services available in some districts. A challenge admitted by policymakers is the poor application of economic evaluations to inform prioritization of reproductive care services (Table 6.4, $\mathrm{p}^{10}$ ). This offers a challenge in estimating effectively ongoing targets for reproductive health interventions at the public-private health sector level. Community level actors such as chiefs/opinion leaders contribute little to reproductive policy setting at their facility according to women (Table $6.4, \mathrm{w}^{5}$ ). Health staff stated that decision making for protocol dissemination of reproductive services is inadequate. A health staff recalled how they have sometimes to demand sometimes refresher training to understand some reproductive health working protocols (Table 6. $\left.\mathrm{hs}^{8}\right)$. 


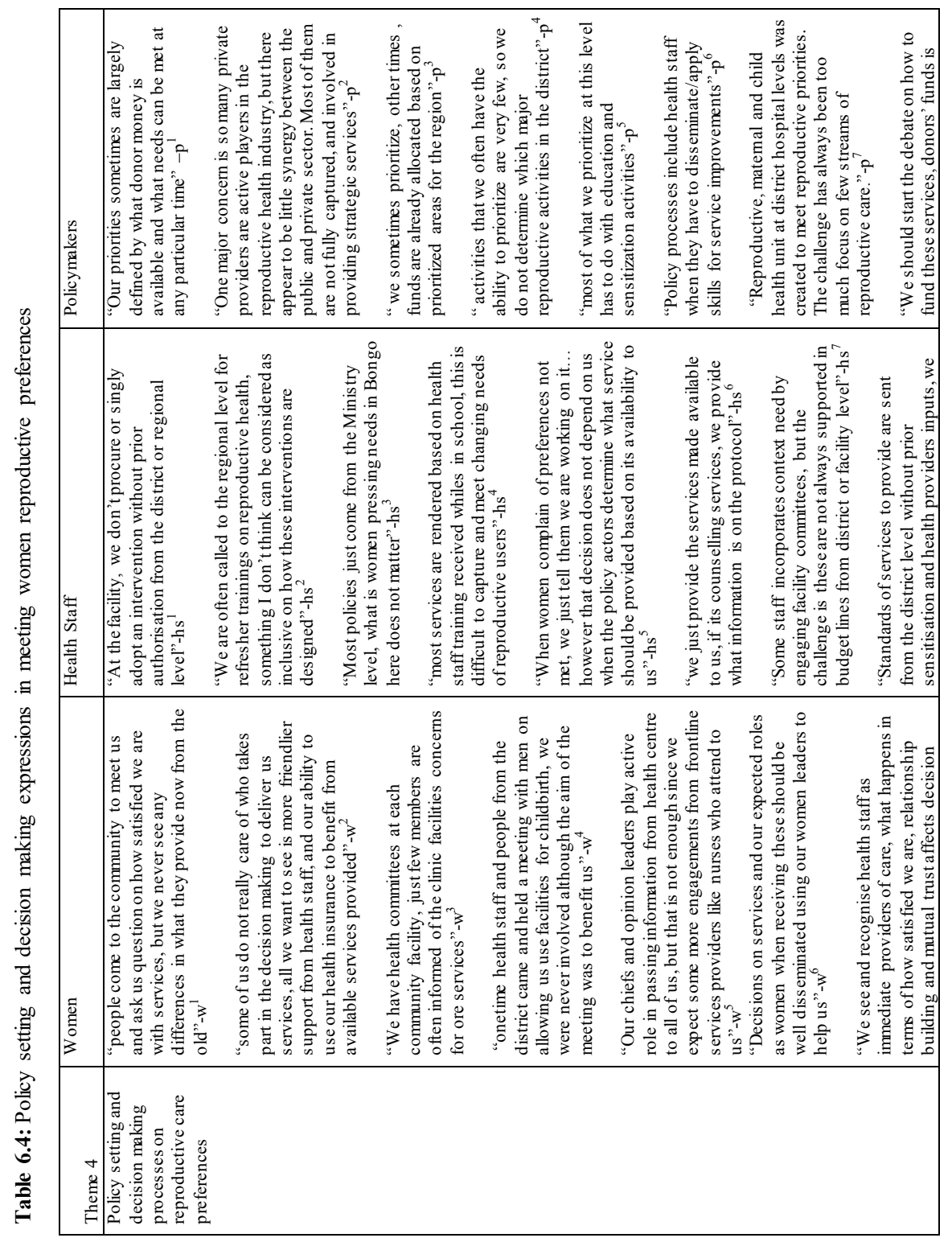




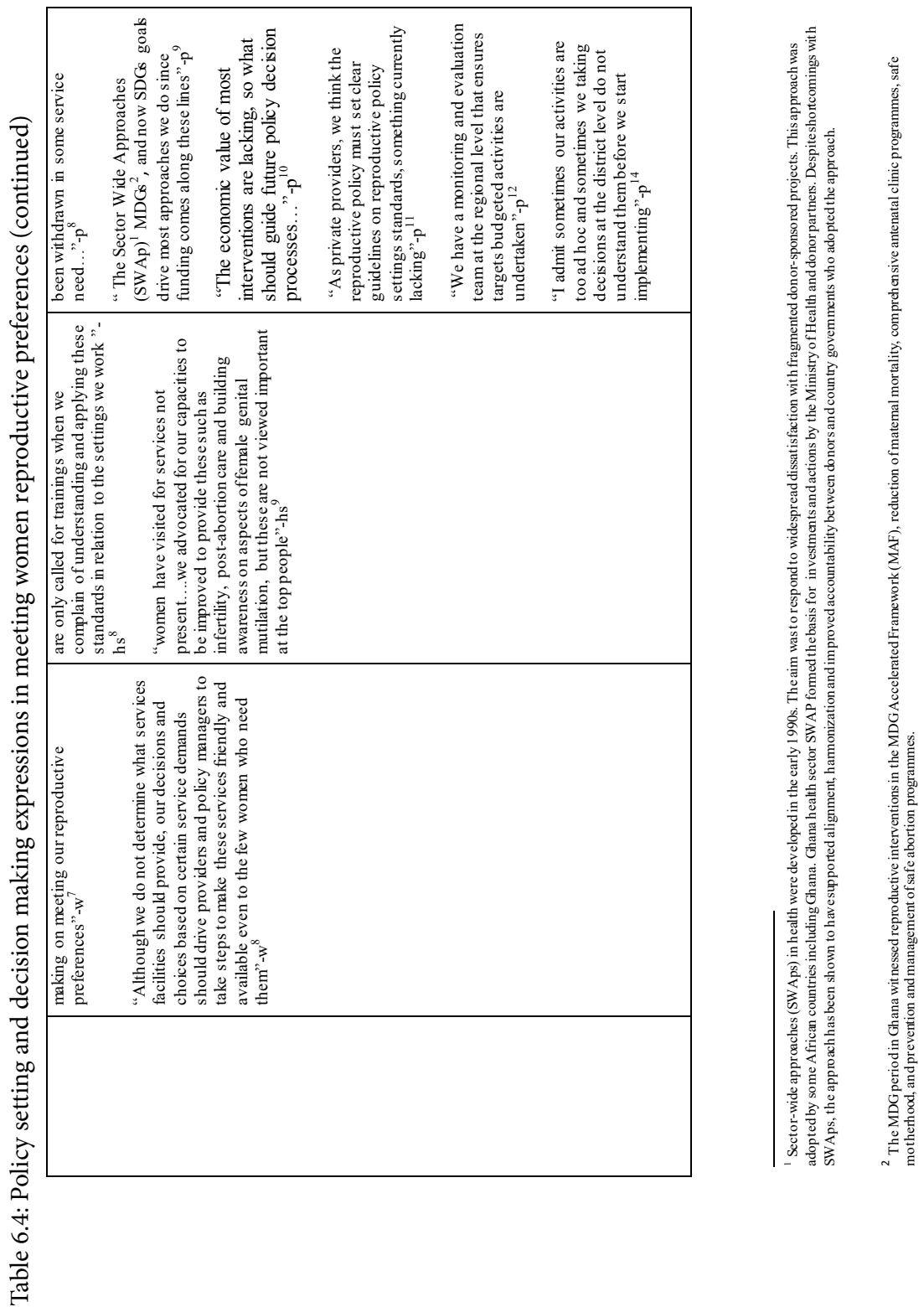


Overall, women recognized that their involvement in reproductive policy decision making was little. Women highly rate their ability to receive friendly and prompt reproductive services compared with their direct involvement in policy setting for reproductive care at the facility level. Also, despite the direct role of health staff in providing services, policy processes on service delivery protocols still is top-down. Health staff observed that their influence on women reproductive care choices is based on conditions that influence services use at the facility level. Most health staff admitted that some services are largely not provided because of logistic problems. All private policymakers strongly agreed that reproductive health targeting must be broad with clear guidelines.

\subsection{Discussion}

This chapter examined the stakeholder's views related to experiences, expectations, and policy decision making processes that influence reproductive care in three rural district settings in Ghana. In addition, we present women's perception of how gender roles influence reproductive care. While women's access to and use of reproductive care services may be influenced by broad social and economic determinants, the experiences, expectations, and policy processes enumerated by all stakeholders' in our findings impact rural women reproductive care choices in rural Ghana.

\section{Gender roles impact on reproductive care}

Our findings on gender roles reflect women views on how gender influences reproductive health care in many societies in SSA. Although many women admitted that male sexuality values have changed over time, this is limited to the family level where women's contribution to economic livelihood is strong. Our findings corroborate several gender studies in developing countries that show that the women's ability to exercise control at the household level improves their chances to use contraceptives and facility-based birthing services (Beegle, Frankenberg, \& Thomas, 2001; Corroon et al., 2014; Mahapatro, 2012). Our findings also show that male perceptions on sexual passivity of women drive male beliefs and desire to control women sexual behaviors. In situations where male spousal control neglects individual women needs, covert use of reproductive care services exist. This finding is consistent with other covert contraceptive studies (Ajah, Dim, Ezegwui, Iyoke, \& Ugwu, 2015; Biddlecom, Munthali, Singh, \& Woog, 2007; Mahler, 1999). Many women agreed that men's role transects across abortion, pregnancy and childbirth, and infertility decision making. 
This corroborates anthropological studies that examined men's multiple roles and influence on women's reproductive health (Dudgeon \& Inhorn, 2004).

Women report that health staff often used cluttered control mechanism in the provision of services. This may indicate a structure of social networks existing between health care providers and users of health care services. One study distinguishes power in sexual reproductive relations where one form of power has negative and limiting authority over others ability to effectively utilize reproductive services (Blanc, 2001). In this study, health staff exercise of control and supremacy has the tendency to shy women away from meeting their reproductive care needs. Systems and structures that create conditions of mutual respect among provider-user, and empower women in their healthcare seeking behaviors need to be encouraged.

\section{Experiences related to reproductive care needs}

Our findings show that non-differentiation at the point of service provision influences individual reproductive choices and use. This finding shows that women with high parities may prefer reproductive care services different from that of adolescents. Our findings on the need for service differentiation support the policy to provide reproductive services by taking into consideration the socio-demographic background of users and the group or social milieu of the intended user (GOG, 2000). Services targeting general reproductive care in any population requires recognition and prioritization of different population group levels (Binka, Nazzar, \& Phillips, 1995).

At the policy and provider levels, participant's views advanced the need to prioritize health infrastructure design and facility friendly centers to cater for individualized reproductive needs. Overall, women reported fear of being stigmatized if services do not differentiate between elderly and younger women. Additionally, social stigma contributed to women reporting poor experiences when seeking abortion care for their adolescent girls in this study. Existing studies support that adolescents girls have unique sexual care needs, are susceptibility to risky behaviors, and more likely to be exposed to inaccurate information on reproductive care services (Åhman \& Shah, 2011; Baschieri et al., 2013).

Another important expression of reproductive experiences in this study is the fee payments for post abortion care and management at the facility level by users. Stakeholder expressions here showed that full fee payments were demanded before a patient is attended for pregnancy loss/miscarriage. Although some policymakers and health staff acknowledged this, they sought to provide reasons why these demands exist. Policymakers and health staff views expressed here however did 
not indicate a justification of these experiences by women. Stakeholder's acceded to a need to fully include post-abortion care and management into the National Health Insurance policy benefit package.

Our findings also show that more social stigma could reduce user's willingness to report poor reproductive experiences at the facility level. Poor experiences associated with contraceptive use in the past may have been influenced by users perceived feelings they were socially insecure regarding contraceptive use. These perceptions have a tendency to lower contraceptive use in an environment where the male spouse determines whether the couple uses contraception. This is also true in existing studies where social stigma is known to lower reproductive services use (Hall et al., 2016; Hanschmidt, Linde, Hilbert, Riedel-Heller, \& Kersting, 2016; Slade, O’Neill, Simpson, \& Lashen, 2007). In situations where reproductive services for STIs, abortion/post-abortion care, and infertility treatments are limited in scope, myths on what defines sexuality and womanhood in the society further foil social stigma on care seeking for such services. Policymakers and provider attestation that some reproductive care services remain unmet and are not currently provided indicates that financial resources need to increase and better target the needs of women.

Stakeholder similarities in experiences converged on the limitation of reproductive care components provided at most of our study facilities level. Stakeholder consensus views here expressed the need for more facility-based resource allocation for meeting programmed facility-based reproductive plans. Health staff views sharply differed from women assertions services provided were non-differentiated, citing limited facility spaces and more health staff as reasons perceived by women that they were not provided differentiated services.

\section{Expectations on reproductive care needs}

Appropriate reproductive information to manage health side effects, particularly contraceptives use, was largely expressed by women users. The results reveal that reproductive services are dealt more with counselling and product delivery for family planning and neglect other essential components of reproductive care. The management of opportunistic infections such as reproductive cancers and infertility treatments desires were seen as lacking in the services delivered. Health staff affirmation that most reproductive services involved family planning, suggesting the inadequacy of appropriate diagnostic and treatment infrastructure have long been documented as a major barrier in delivering maternity care services in Ghana (MOH, 2008, 2011, 2014). Policymakers and health staff acknowledgement that private health providers were active players in delivering a broad range of reproductive services is an admittance of shortcomings at the 
public provider level. Since private facilities tend to be more urbanized, rural women seeking abortion and infertility treatments face distance barriers aside existing social stigma and norms for these services.

Another repeated expectation among all stakeholder groups was the need to improve systems that are more integrative and supportive for reproductive service users. In this study, individualized reproductive care was admitted by policymakers and health staff as difficult to attain, partly due to limited health staff to provide services. This reason could explain why most health staff have not been equipped and trained appropriately to provide other broad reproductive services such as fertility counselling and treatments and abortion care. Our finding on stakeholder's positivity in delivering integrated reproductive services, and its associated barriers is consistent with other studies in Africa (Dehne \& Snow, 1999; Maharaj \& Cleland, 2005; Mutemwa et al., 2013).

This chapter provides evidence that the non-availability of psychological support creates low self-esteem among intended users. While women expressed optimism that information and counselling enabled them to address clinical reproductive concerns, these counselling services were limited in supporting them to address individual psychological needs. The inability of the formal health system to address these specific psychological stress needs has a propensity to lower or withdraw reproductive care services. Our finding here corroborates existing findings that a psychological burden is the most common reason for patients to withdraw from a facility treatment centre for infertility (Domar, 2004).

Lastly, adopting participatory approaches for reproductive services delivery is acknowledged by all stakeholder groups as vital for improving reproductive services. The mother-to-mother support (MTMS) group evidence from our study draws more evidence of the important role played by informal community structures to promote health and well-being. Our study typifies studies across developing countries that support bottom-up approaches to deliver community health services (Farmer et al., 2010; Opit, 1987; Rifkin, 1986). It also reflects evidence from other studies that specifically view informal groups as an integral component to provide satisfying health care needs in rural populations (Bawah, Akweongo, Simmons, \& Phillips, 1999; Kiapi-Iwa \& Hart, 2004).

Stakeholder's expectations in our study differed on what levels of counselling were adequate to meet user reproductive care expectations. Women viewed most counselling services as inadequate, and not tailored towards individual needs. Health staff and policymakers however were of the opinion that current counselling services were adequately designed, stressing that these counselling services were only limited due to low health staff to patient ratios at health facilities. To ensure current health sector policies on counselling meet user expectations, 
there is a need for consensus among key stakeholders on counselling components for reproductive care services.

\section{Policy setting and decision making processes}

The policy processes for informed decision making on reproductive care services is driven by donor funding, with policymakers and health staff acknowledging difficulties in prioritizing to meet local reproductive care needs at facility levels. Despite the health sector policies to ensure bottom-up planning processes, views from policymakers and health staff on reproductive care services indicate bottom-up planning gaps. The two stakeholder groups above stated that few reproductive activities are budgeted and supported by health insurance reimbursements, acknowledging that irregular insurance reimbursements leaves most facilities ineffective. An existing study on priority setting on sexual health in Ghana shows that reproductive policy setting is rudimentary, with little involvement from donors and advocates (Mayhew \& Adjei, 2004). While our study did not investigate donor funding sources and donor involvement in reproductive care, our results from all stakeholders indicate a weak involvement on reproductive planning process on products and services delivery among stakeholders.

Women indicated little involvement in policies on their expected needs, despite their involvement in health committee meetings with facility and district health providers. Agreeably, health staff also indicated most reproductive policy decisions come from the ministry, with little space for other stakeholder inputs. Health staffs influence on women reproductive care choices based on child birthing conditions at facility level, and not through involvement in policy processes on reproductive care. Health staff views blamed policymakers at district levels for failing to recognize user concerns on policy processes on reproductive care services. Policymakers disagreed with these assertions, indicating deficits in health sector funding and logistical needs account for perceived inefficiency at the district levels. Furthermore, policymakers insisted reproductive health targeting still remain limited in scope, since most reproductive care guidelines and protocols have not been contextualized and fully operational in all facilities.

Some major policy processes and decisions among policymakers in our study have been driven by political commitments over the last decades. Specifically, many safe motherhood interventions implemented during the MDG period were influenced by political reform processes. One such policy is the free fee exemption policy for delivering care and the National Health Insurance Scheme in Ghana. These policies provided impetus for many health sector goals for improving sexual reproductive health needs in Ghana (Phillips, Bawah, \& Binka, 2006; Witter, 
Adjei, Armar-Klemesu, \& Graham, 2009). Policymakers in our study noted poor application of economic evaluation to evaluate earlier SWAp processes. This view corroborates exiting studies in Ghana that indicated misinformation and poor stakeholder understanding greeted some sector polices on sexual and reproductive health (Mayhew \& Adjei, 2004; Seddoh \& Akor, 2012). Evidence from policymakers in our study also points to the poor application of economic evaluations to inform reproductive priority setting and decision making over the years. This suggests the need for reproductive systems to adopt approaches to measure policy impacts over time to ensure policy process not only are inclusive, but contribute to improving reproductive health outcomes.

This study has some limitations. First, notwithstanding the saturation observed in our interviews, we may have still missed out important categories that could have emerged among stakeholders in our study. We also acknowledge the possibility of health staff and policy implementer's biases in trying to withhold information on the study aim. Our triangulation of views have minimizes potential errors on stakeholder narratives. We acknowledge the inability of our study to investigate views from persons at the health ministry level and other public-private partners involved in implementing reproductive health strategies. Nevertheless, the three groups of stakeholders examined in this study represent a good fit when examining district level stakeholders on sexual and reproductive care needs in any rural context in Ghana.

\subsection{Conclusion}

This chapter explored stakeholder's views on experiences, expectations, and decision making on reproductive care among rural Ghanaian women. Gender mainstreaming approaches in Ghana should develop structures and systems within the health delivery system that create conditions of mutual respect among provider-user, and empower women in their healthcare seeking behaviors. In addition, health teams working to improve sexual and reproductive health care must find suitable strategies that will effectively work to improve women reproductive care needs at their operational levels. From our results, the accounts of non-differentiation of services among adolescents and older women group's calls for the need for health providers at facility levels to adjust health services to target and meet specific needs for different user groups. While we support the inclusion of post-abortion care and post-pregnancy termination management issues into the National health insurance benefit package, a broad program scheme that ensures sexual and reproductive health needs are truly subsidized or provided essentially at lower fee cost will benefit rural population groups. 
To support accountability and equity in reproductive services provision at facility levels, the local health systems need to be resourced to evaluate health staff working attitudes in relation with user needs and satisfied reproductive care demands. To upscale services using the private sector contributor approach, policies that target increased private sector participation may result in more multifaceted reproductive services care provisions, something not entirely available in most public sector health facilities.

Health sector leads such as the Ministry of Health and Ghana Health Service should drive the need to close the inclusiveness gap on sexual and reproductive care sector policy planning processes and needs. Stakeholder consensus from this study also strongly advocates clear guidelines on reproductive care to be contextualized to meet user care needs. Health counselling and the need for individualized care have increasingly been evidenced as an entry approach to reach new users of health services. To fully reach individualized care at community level, health systems must have community support clutches such as the motherto-mother support groups evidenced from our study. This is important to provide community level psychological support and delimit cluttered relationships that may exist between provider-user during formal facility access hours. 



\section{CHAPTER 7}

General Discussion 


\subsection{Introduction}

The principal aim of this dissertation was to increase our understand ing of unmet maternity and reproductive care service needs over a woman's life course in rural Ghana. Ghana has high unmet reproductive health needs (GSS et al., 2014) (29.9\% for family planning, and 319 per 100,000 live births for maternal mortality rates). Despite a high rate of modern contraceptive awareness, unwanted pregnancies, unsafe abortion and inadequate birthing services abound (Baiden, Mensah, Akoto, Delvaux, \& Appiah, 2016; GSS et al., 2014). To address unmet needs for reproductive health services in Ghana, and across SSA in general, strategies and policies must anchor reproductive health services delivery. The adequacy of policies and measures to meet targets on unmet reproductive care needs remain largely unexplored across West Africa. A critical examination of measures and determinants of reproductive health care provision and utilization is a first step to improve maternity and reproductive health outcomes. As indicated in Chapter 1 , to improve women's reproductive health service needs in rural Ghana, it is also important to understand unmet reproductive care demands. Studies on contextual barriers to reproductive health care in Ghana exist, but few of these studies have examined the concept of unmet needs broadly and in multiple heterogeneous rural districts in Ghana. To understand determinants of reproductive care, an assessment of the social, economic, and environmental factors that drive women's productive and reproductive years was examined. At the policy level, our findings reflect shifting needs for patient-centred care practices not only for reproductive health care needs, but for the entire health care system in Ghana.

To address the aim of this dissertation, three research questions were formulated as outlined in Chapter 1. First, there is little evidence available on the adequacy of outcome measures and determinants for women unmet reproductive health needs. A review of the outcome measures and determinants of unmet reproductive health needs among women in West African was presented in Chapter 2. To provide empirical evidence, we undertook a systematic review of recent studies published in academic journals (from January 2009- March 2014). Outcome measures and determinants were then assessed along three main reproductive service needs: contraceptive use, obstetric care and ANC utilization. Second, in Chapter 3 and Chapter 4 of the dissertation, we explored focused maternity care and stakeholder opinions of maternity care shortcomings in rural Ghana respectively. In Chapter 3, we provided an analysis of women's experiences with maternity care and their met and unmet reproductive health services. The assessment was benchmarked against WHO standards for prenatal and postnatal care. 
Using data from the 2008 GDHS, and applying a cluster analysis approach, we examined the adequacy of focused maternity care in Ghana. In Chapter 4, we further used ethnography to examine stakeholder perspectives on shortcomings for maternity care. We contrasted the views of women, health staff and health policymakers on shortcomings for maternity care in three rural Ghanaian districts. Lastly, we have taken a look at context factors and the role that reproductive health service preferences play in reproductive health services provision and use. In Chapter 5, the importance of context-specific associations for women contraceptive use was examined. Using descriptive statistics and a cross sectional design in a heterogeneous setting in rural Ghanaian district, we described the multitude of women unmet contraceptive desires and use. We emphasized in this Chapter the need for focused counselling and a health care system that prioritizes improvements at clinic level arrangements for contraceptive services uptake. In Chapter 6, we also examined the experiences, expectations and decision making processes among stakeholders across three rural districts of rural Ghana. In Chapter 6, we further shed light on how local health systems can improve accountability and support for delivering reproductive care services in rural Ghana. In this final chapter of the dissertation, we present and discuss the main findings of the dissertation in the form of six key statements.

\subsection{Discussion of statements}

Statement 1: Reproductive health targets like service quality and user satisfaction are not only reproductive outcome measures, but also important determinants of reproductive health service use among women in West Africa.

As argued in Chapter 2 of this dissertation, identifying and applying relevant reproductive health targets should be country specific. These context specific targets provide opportunities to evaluate country level progress on reproductive health care needs. The identification of country specific reproductive health services needs help to design interventions that improve women's chances for reproductive services utilization. In the introductory chapter of this dissertation, we posited that the application of any global target should reflect needs. Our findings confirm that the current targets for women's reproductive health needs are inadequate if applied broadly (Chapter 2). Specifically, reducing unmet needs for family planning as a reproductive target should account for the socio-cultural environment in which the target is investigated.

Service quality and user satisfaction remain important drivers for women reproductive health service utilization in West Africa (Chapter 2). Reproductive 
service quality and user satisfaction determine reproductive care services use. More specifically, clinical outcome estimates from our review show that a positive evaluation of skilled care received influences the use of maternal and reproductive care services (Chapter 3). This dissertation further shows that, quality related reproductive care outcome estimates are largely documented at the clinical and patient level as evidenced in Chapter 2. We posit that poor maternal care quality often exists where health sector reforms do little to improve social infrastructure (Chapter 4). We found that, a woman's interfamilial surroundings influence quality ratings for reproductive care services (Chapter 2). Such interfamilial surroundings include attributes such as a woman's perceived quality of life and perceptions of health care provider's knowledge levels.

User satisfaction has long been seen as an important component of service quality (Sitzia \& Wood, 1997). As argued by Lohr (1990), targets for health services quality should include patient satisfaction for services use (Lohr \& Schroeder, 1990). As explained in Chapter 2, even among clinical measures for contraceptive use, broad measures such as unmet needs for contraception, adolescent birth rates, and unintended pregnancy estimates rely on users' satisfaction proxies. Our findings show that reported quality improvements for reproductive care have context causes. Thus, social, economic, and environmental determinants need to be integrated into country level service delivery strategies (Chapter 2, 4 , and 6). We emphasize that reproductive decision making and place of service utilization influence women use and satisfaction of available services. Where service provision does not match women's demand, women's future use intentions are often unclear (Chapter 4 \& 5). However, evidence supports the notion that a client's satisfaction with health service needs is associated with a patient's intention to use clinic services in the future (Alden \& Bhawuk, 2004).

This dissertation also established that, reproductive care quality and satisfaction have the tendency to determine reproductive care use and caring practices received from health care providers (Chapter 2). Poor service quality and user satisfaction may facilitate the existence of unmet reproductive health care. Thus, poor quality and lowly rated reproductive care services exacerbate reproductive unmet needs. In West Africa, service quality and user satisfaction reflect the inability of the health care system to meet women's demands for reproductive care. Socio-demographic factors such as income, education, age, and residence type are the main determinants of reproductive care disparities across West Africa. Furthermore, in West Africa, differences in country level fertility policies and good governance influence demand for reproductive services (Chapter 2). In addition, normative socio-cultural beliefs and social roles interact and shape reproductive health behaviors, choices and demand among women in West Africa. There is evidence that social norms and culture influence women's 
perceptions of needs as well (Bove et al., 2012; Brazier et al., 2009; Eliason et al., 2013; Izugbara \& Ezeh, 2010). As discussed in Chapter 5, intentions of young women for contraceptive use in the future depend on social and group norms. In settings where women covertly use reproductive care services, user satisfaction drives current and future reproductive health use intentions (Chapter 5).

We conclude that in developing countries, many aspects of inter-personal care such as user satisfaction and quality influence maternal health outcomes. Furthermore, health care provider's gender contributes to maternal care satisfaction (Srivastava, Avan, Rajbangshi, \& Bhattacharyya, 2015). The provision of culturally sensitive care is known to improve maternal health outcomes and maternal care satisfaction in developing countries. Culture and care dynamics and interrelationships need to be investigated to improve service provision in rural areas. The quality of maternal care and user satisfaction need to become part of the structure underlying maternal care provision in developing countries. There is a need for systemic mapping of causal pathways between cultural context and technical interventions that impact on maternal care outcomes.

\section{Research and policy implications}

Reproductive health care targets must reflect country needs. This will better address disparities in maternal and reproductive services use. In line with this, there is a need for further study to explore country specific factors related to reproductive service quality and user satisfaction. The evidence from our review showed no direct association between user satisfaction, and service quality, despite the two being major drivers for reproductive services utilization. Future studies should identify broad determinants for reproductive service quality and user satisfaction. A specific focus on increasing the knowledge base of quality and satisfaction attributes for reproductive service needs could better inform reproductive program planner's strategies when intervening on delivering integrated reproductive care services.

In addressing service quality and user's satisfaction in countries in West Africa, tailored policies to improve poor health systems for reproductive care service delivery are required. Health system arrangements that create reproductive health friendly centers while addressing unsatisfied demands may help upscale reproductive services use in rural settings. At the political level, policies must address social, economic and environmental factors along the continuum of care for vulnerable women in developing countries. Target driven and cost effective interventions that ensure services are delivered in socially accepted modes, enhance user's expectations and chances for future services use. Health reforms that improve reproductive health access (especially legislative reforms that restrict/ 
limit reproductive care) could improve current unmet reproductive needs. The continuous generation of reliable data to support the delivery of reproductive and general health services delivery is critical. Good health data are important to inform on effective policies in delivering reproductive health services. In addition, reliable and efficient health data promote good governance and policy decisions among all stakeholders involved in promoting better reproductive health outcomes.

Statement 2: Focused reproductive counselling and clinic setting arrangements facilitate contraceptive access and use among rural Ghanaian women

Focused reproductive counselling is critical to scale up family planning and broad reproductive health services among women in Ghana (Chapter 5). Focused counselling and clinic arrangements are exemplified in this dissertation as important to ensure access and use of contraceptives. Furthermore, focused reproductive counselling contributes to addressing rural women's access and use of contraception. A previous study in Ghana has suggested that traditional norms and misconception of contraceptive use influence a woman's life choices on child bearing (Parr, 2003). We established in this dissertation that, even among women without formal education, focused reproductive counselling help to address contraceptive misconceptions, myths and wrong cultural norms on fertility control among rural women in Ghana (Chapter 5). Many other socio-cultural and economic factors also influence reproductive choices along the reproductive continuum. The provision of timely and tailored reproductive counselling services increase women's awareness and control over hormonal effects during contraceptive use. Its effectiveness also accounts for postpartum contraceptive choices in later life (Eliason et al., 2013).

Focused reproductive counselling affects women's contraceptive decision making in two ways; 1) Focused reproductive counselling has the propensity to enhance women's contraceptive autonomy by expanding their productive choices and reproductive health care use. Our evidence of contraceptive use in Ghana show rural women's willingness to expand their reproductive health choices when they feel economically empowered and part of the general health delivery system. Studies show focused reproductive counselling enhances women reproductive decision making and fertility choices (Corroon et al., 2014; Izugbara et al., 2010; Metcalfe, 2010; Rottach et al., 2009). For many vulnerable population groups, focused reproductive counselling provides an alternative to obtain focused maternity care (Chapter 5). The provision of adequate focused reproductive counselling services addresses covert users' needs for confidential and patient-centered care. Quantitative and qualitative results on focused 
reproductive counselling show a divergence in the effects of focused counselling on future contraceptive use intentions among rural Ghanaian women (Chapter 5). A further examination of these groups' socio-demographics such as parity and educational levels, family union type, and normative structures, could provide explanations for these differences. Given that rural uneducated married women in polygamous unions in Ghana use contraceptives rarely (Ayanore \& Aryee, 2015), focused counselling may address contraceptive user's health concerns. This has the potential to reinforce positive contraceptive behavior among them. 2) Prompt and adequate focused reproductive counselling can assist health staff and user's shared decision making on reproductive care service use. Shared decision making is known to further enhance long term fertility goals as espoused by the principle of the "calculus of conscious choice". Additionally, our findings on clinic counselling services should move beyond the health system and recognize the social patriarchal dimensions for reproductive service delivery. Men willing to support facility level health staff who deliver contraceptive services enhance family planning social capital for contraceptive use.

Clinic level arrangements explain start-stop attitudes on contraceptive use among rural Ghanaian women (Chapter 5 and 6). Women start, continue, or make alternative fertility options because of strict rules prescribed by health care providers (Chapter 5). Evidence also shows that individual intentions for contraceptive use may not reflect actual use (Chapter 5). Given that existing barriers could prevent women from attaining their desired contraceptive intentions, client services that provide users with multiple choices improve reproductive care utilization. Contraceptive choices delivered through focused counselling at friendly clinics reassure and address users' needs (Chapter 5). In clinics perceived as unfriendly by women, counselling services have low acceptance levels. This is true for rural women who posited that health staff "scanty" information and open censures often do not address individual concerns on contraception (Chapter 5). Another significant clinic level dimension for contraceptive access and use is the provision of appropriate hour's services at the convenience for rural women. For most rural women, appropriate clinic service hours enable them to use services all year round, irrespective of seasonal and environmental conditions. The evidence showed that women prefer outreach services including home visits by health staff when social and environmental conditions makes them difficult to access and use services (Chapter 5).

Overall, studies support that clinic level improvements that value patient concerns and are community-based and integrated effectively into a functioning health systems deliver effective maternal health outcomes (ten Hoope-Bender et al., 2014). Neglecting technical standards in infrastructure, and infection control strategies may undermine quality standards for maternal care. Focused 
counselling is problematic in many contexts because of several single reproductive and maternal care services are provided independently. Integration of reproductive health care services and quality of consultation for reproductive health services are essential components of health sector reforms. These multiple strategies enumerated above remain essential and integral in delivering and attaining better maternity outcomes across many developing context.

\section{Research and Policy Implications}

Quantitative findings presented in in Chapter 5 show that current contraceptive users with prior focused counselling had less intention to use contraceptives in the future. Further exploration in a large heterogeneous rural Ghanaian population using quantitative and qualitative data could provide evidence of the impact of focused counselling on reproductive behavior. Additional study is needed to assess the effects of the frequency of contraceptive counselling services, what strategies best work in clinic settings during counselling services, and counselling periods that influence contraceptive access and use. Documenting women's experiences and preference on contraceptive counselling services is necessary to upscale services use.

To address women's contraceptive needs, clinics must design structures that guarantee not only confidentiality, but suitable working hours that enhances women access and use of services. Furthermore, the provision of reproductive care services must be more client-centred, and respond to culturally sensitivities of different population groups. Services that aim at educating and addressing user health concerns should be more targeted to provide better reproductive health outcomes. Thus, health system processes that allow providers to incorporate focused counselling and educational support will impact on women contraceptive access and use. Clinic level procedures that guarantee privacy and support male spouses who are willing to support these services are needed as well. Policies to improve contraceptive use should consider the examination of user preferences, expectations, experiences and decision making involvement that guarantee users willingness to use desired services.

Statement 3: While health insurance reduces the financial risks and barriers for focused maternity care, the lack of health insurance further increases indirect cost and negatively impacts maternity care outcomes among Ghanaian women.

A woman's chance to report early at first antenatal, interested to seek repeated episodes of maternity care is influenced by her having health insurance. We find that, indirect cost and poor access to focused maternity care is mediated through 
health insurance (Chapter 3). Thus, having health insurance play a significant role in the cost (indirect) and care adequacy among women in Ghana. In Chapter 3, we show that women who are poor (people in poorest wealth quartiles) have a high propensity to lack health insurance. Thus, these women are more likely to obtain least adequate care (Chapter 3 ). Even among insured groups in Ghana, health care seekers may often use out-of-pocket payments as a means to obtain friendlier and supportive maternity care services (Chapter 4, and 6). In the absence of health insurance, out-of-pocket payments tend to impose a high financial burden on the poor and more specifically, on those in low wealth quartiles to access services (Habicht, Xu, Couffinhal, \& Kutzin, 2006).

Insured women are more likely to use maternity care more than once in Ghana as reported in this dissertation. This confirms existing evidence that having insurance is positively associated with health care usage (Scheil-Adlung, Carrin, Juetting, \& Xu, 2006). The evidence shows that having social health insurance makes people more willing to seek formal care, especially their willingness to accept hospitalization when ill (Blanchet, Fink, \& Osei-Akoto, 2012). Country variation in poverty and low socio-economic opportunities present barriers to use maternity care everywhere. Our cluster analysis findings for prenatal and postnatal care reinforce that regional variation and health insurance interact and affect the adequacy of focused maternity care in Ghana. In Chapter 3 of this dissertation, we established that insurance and its attendant effects are influenced by geography. We find, specifically that regional variation in health insurance access and use influence disproportionally the adequacy of maternity care across regions. Our study on regional variation show differences in old, widowed or divorced women experiences of adequate care. This may suggest that these vulnerable groups (older and single marital status) may be less likely to receive focused maternity care. Three out of 10 regions (Volta, Upper West, Northern and Western) which are classified as the least poor in Ghana had more women reporting less adequate care. In environments where women report travel time and financial cost barriers for care seeking, there is greater disparity in general health use. This is hardest among women living in rural dwellings. Our results also highlight that poorer population groups are impacted negatively by indirect cost and poor quality attributes for maternity care in Ghana in a cross country study (Odeyemi \& Nixon, 2013).

Although the insured will more likely use health services at times of need, insured individuals may at times use more services than required (moral hazard). However, the higher health care use may not necessary reflect the ability of insurance card subscribers to obtain adequate and timely care. The inability for improvised socio-economic groups across these improvised regions to obtain health insurance year after year explains why less adequate care is reported in 
these regions (Chapter 3). The catastrophic effects of not being insured further reduce the abilities to obtain adequate and quality maternal care services.

More so, macro-level evidence of health insurance ownership in Ghana show more beneficial equity developments (private health expenditure decreased by $14 \%$ ) in the last decade. Additionally, many micro-level inequities (vertical inequity) exist for maternal care access and utilization (Odeyemi \& Nixon, 2013). Complementary social health protection schemes are known to positively moderate insured people's use of general health services in Africa (Scheil-Adlung et al., 2006). Health systems that invest in social protection and health insurance schemes improve the likelihood of insured people to use available health services. This suggests that health insurance or the lack of health insurance alone, may not guarantee focused maternity care. Targeted social protection interventions among insured and non-insured vulnerable population groups could improve focused maternity care among rural Ghanaian women.

Additionally, the lack of health insurance further aggravates indirect cost and the adequacy of care for many Ghanaian women. Indirect cost for accessing maternity care is influenced by broad social, economic and infrastructural deficits in many rural areas in Ghana (Chapter 4, 5 and 6). As households face several risks (health risk, protection risk etc.) to access health services, the absence of health insurance can further reduce the ability of vulnerable groups to obtain adequate care (Scheil-Adlung et al., 2006). In advocating continuous expansion in health insurance as a means to improve the adequacy of care, health systems in developing countries must address skill workforce shortages and measures that improve women choices and active participation in maternity and reproductive care services.

In conclusion, health insurance provides potential benefits for prenatal and postnatal facility utilization especially among rural residents in Ghana. In Chapter 3 and 6 of this dissertation, we advocate a more broad based inclusiveness on the health insurance benefit package along a continuum of maternal care services. Insured individuals may still experience financial constraints to access care if health insurance benefit packages remain limited in scope. Essential components of focused maternity care espoused by WHO will remain minimally attained among many population groups in the absence of functional health insurance and complementary social protection schemes. Health insurance benefit packages that do not address broad societal risk and vulnerability issues will do little to improve access and the adequacy of care for vulnerable groups. 


\section{Research and policy implications}

To improve the adequacy and access of focused maternity care, there is a need to identify the right nuxus of the effects of health insurance on health seeking. Some insured population groups may not use health services, even when in need, hence, the need to find context drivers for these differences. In Ghana, there is a need for data and evidence on how to improve the dissemination of policy guidelines on maternity care, especially in rural health facilities. Although several studies have evaluated social health insurance and its effect on maternal care utilization, few studies have assessed the adequacy of care in relation to indirect cost and quality. Future studies are encouraged to examine these into greater detail to unearth policies that better target vulnerable groups ability to access services with or without health insurance.

In Ghana, health insurance provides potential benefits for prenatal and postnatal facility utilization especially among rural residents (Chapter 3). Improving structural deficits under the current national health insurance scheme will inject more confidence for users, and non-active members (Chapter 3). We suggest the revision of the insurance type and benefits packages, with target premiums that improve pro-poor household's ability to access a continuum of care for women after birth. Current reforms in the health insurance must integrate value-based health care that seeks to provide focused maternity care among vulnerable population groups. Additionally, broad health system improvements such as increased training and increased motivation for rural health staff contribute to better health outcomes. Clinical care conditions and those that ensure cultural sensitivity on maternity care delivery are also critical to improve the adequacy of maternity care (Chapter 3, 4 and 6). Evidence suggests that vertical programs are often poorly implemented with structural deficits and much political influence that do not contribute to accountability to beneficiaries (McPake et al., 2011; Ridde \& Morestin, 2011; Singh et al., 2003). We suggest that long term financial planning to meet the needs of short-gap vertical health programs. In Ghana, there is need for consistent forecasting and financial planning on vertical interventions to improve the adequacy of maternity and reproductive interventions. A coherent maternity care policy plan that creates greater accountability for quality, equity and geography (region and rural residence) is needed.

Statement 4: The number of antenatal visits made by pregnant women does not ensure that adequate care is received

An important element in the continuum of adequate care for women and children globally is how effective the ANC system is organized (Lincetto, 
Mothebesoane-Anoh, Gomez, \& Munjanja, 2006). ANC coverage is proven to be a success story in Africa, with over two-thirds of pregnant women (69\%) receiving a minimum of one ANC visit during pregnancy (Lincetto et al., 2006). While ANC interventions, in and of themselves alone cannot drastically improve maternity health outcomes, they remain essential to attain perinatal and child survival outcomes in any context (Abou-Zahr et al., 2003). More so, ANC remain a critical entry point to intervene in ensuring a healthy continuum of care for mother and baby.

This dissertation postulates that focused care advocated by WHO (with a minimum of 4 visits to facility) by a woman may not guarantee adequate maternity care, if supply related factors are neglected in the provision of ANC. In this dissertation, we use cluster analysis to segregate the adequacy of prenatal and postnatal care received by 2,147 women (763 urban and 1,384 rural women) within reproductive age (aged 15-49 years) across all regions in Ghana (Chapter 3). ANC patterns investigated were categorized as adequate, less adequate and least adequate. The results from our study show that adequate ANC is not associated with the frequency of visits made to the facility by a woman during pregnancy (Chapter 3). The quality and adequacy of care is however positively associated with health care support received (skilled and female attendant at ANC). In addition, the ability of women to overcome supply related barriers such as travel cost and poor access, lack of health insurance also influence maternity care adequacy (Chapter 3). As outlined in Chapter 1, the frequency of antenatal visits differs depending on health risk and environmental conditions that surrounds a woman's reproductive life course. This view reflects other findings that the optimum number of ANC visits for women in poor settings depends on costs and other barriers for ANC access (von Both, Fle $\beta a$, Makuwani, Mpembeni, \& Jahn, 2006). Overall, age, parity, regional variations, residence type (rural/urban) and not being covered by health insurance were found to influence the adequacy of a woman's ANC (Chapter 3 ). The above factors influenced more positively a woman's adequacy of prenatal care irrespective of the frequency of visits made to the health facility (Chapter 3 ).

In examining the frequency and timing for ANC visits during pregnancy, it is important to focus on what impact the frequency of visits has on the level of ANC quality. Statement 1 of this Chapter, asserts that user satisfaction and care quality is influenced by individual users, social, and supply related factors for care delivery. ANC quality and satisfaction affect maternal care outcomes and are known to influence women's reported prenatal and postnatal care outcomes (Chapter 2 and 3). We however acknowledge that a low frequency of visits or late timing for first antenatal visit limits the amount and quality of care that a pregnant woman receives (Magadi, Madise, \& Rodrigues, 2000). In statement 
1 of this Chapter 7, we revisited the importance service quality play in driving reproductive health service utilization among women in West Africa. We do not have data that directly equate the frequency of visits and quality of ANC in this dissertation.

In stressing the adequacy of visits for women during ANC, it is also important to determine sufficient times allocated for ANC services. Thus, it is important to determine the frequency of visits that remain relevant in improving care quality and user satisfaction. Providing women sufficient consultation times at health facility centers during antenatal addresses inadequate care needs for most pregnant women (Magadi et al., 2000; von Both et al., 2006). Our results in Chapter 3 show that, factors such as age, parity, regional variations, and residence type (rural/ urban) drive care adequacy among Ghanaian women. Fundamentally, insisting on a minimum of four visits for ANC by women with a low pregnancy risk may not be practically feasible in settings where the frequency of ANC visits is influenced by how accessible ANC services may be. As presented in Chapter 3, 4 and 5 of this dissertation, reproductive and maternity care outcomes are intrinsically related to women's ability to meet travel time and costs. Thus, any expected increase in distance or time for a woman with a high risk pregnancy may limit such woman's ability to frequent a health facility. Measures that improve women's opportunity cost (travel distance and time efforts) for ANC visits support to attain adequate prenatal care outcomes are also important for policy consideration.

Our results emphasize the need for a careful application of the WHO recommended minimum of four visits among women with a low risk pregnancy. We acknowledge that, focused ANC should rely more on individual care quality, instead of the frequency of visits made for ANC. Greater emphasizes for ANC should focus on improving the quality of essential ANC components during pregnancy. Broadly, ANC services at policy levels must address cost and social barriers that divest vulnerable group's abilities to access care. Patient-centered approaches that build trust and increases women satisfaction with the health services use improves adequate maternity care needs.

\section{Research and policy implications}

As examined above, this dissertation did not establish a direct relationship between the frequency of visits and ANC quality. This can be a relevant area worth investigating further. Any future research should examine the relationship of antenatal frequency on care quality and the optimum ANC counselling needs to guarantee adequate care in a developing context. 
Policy level improvements for continuous ANC coverage should focus on the quality of individual components of ANC services provided at facilities. Service improvements need to incorporate in-service and pre-service trainings of ANC providers on meeting cultural sensitive and acceptable caring practices. For improved coverage and better targeting, focused ANC care should be user tailored and designed to address specific social and cultural barriers for ANC use among women. There is also a need to develop ANC friendly centers that will actively promote $\mathrm{ANC}$ as an entry point for many reproductive interventions along a women's reproductive life among rural populations.

Statement 5: Meeting women's reproductive and maternity health service needs require multiple stakeholder engagements at several levels

Identifying and applying appropriate models on reproductive and maternity care services provision require working across multiple layers of a cadre of skilled health professionals (Chapter 4 and 6). As mentioned in Chapter 4 of this dissertation, policy directives in Ghana over the decades prioritized the role of health actors in health service delivery. Stakeholder engagement and prioritizations help to understand user's preparedness and demands for quality and satisfactory reproductive health service needs (Chapter 6). In Chapter 6 of this dissertation, we stated that understanding reproductive health needs empowers rural women reproductive care seeking. Partnerships between stakeholders at the health facility level enable facility level evaluations of staff performance that are important to monitor reproductive and other general services delivered. There is a growing concern about the role of informal social support systems tailored towards increasing patient-health staff contact hours.

It is also important to recognize that, health reforms are "processoriented"(Horev \& Babad, 2005) and require several stakeholders' inputs. To generate coherence and positive reinforcement in reproductive health policy, traditional models must evolve towards patient-centered care approaches. In addition, shared decision making models promote inclusive reproductive decision making (Chapter 6). Factors impacting reproductive and maternity care in rural Ghana are multifaceted and complex. This highlights the need to strengthen social conditions that facilitate the delivering of effective reproductive care services. In recognizing the need for broad stakeholder engagement to advance reproductive care, health system barriers need to address reproductive inequalities among vulnerable population groups. Our results indicate weak involvement on reproductive planning process on products and services delivery among stakeholders that fail to address reproductive inequalities (Chapter 6). Stakeholder involvement and policy processes for informed decision making are 
driven by previous and current donor funding needs in Ghana (Chapter 6). Some stakeholders acknowledged difficulties in prioritizing local reproductive care needs at facility levels (Chapter 6). In addition, stakeholder group expectations show the need to improve health systems that are more integrative and supportive for delivering reproductive care services in rural Ghana. Adopting participatory approaches on reproductive services delivery is acknowledged by stakeholders as vital for improving reproductive services. However, little involvement in reproductive policy formulation and implementation is reported by women in this dissertation (Chapter 6).

From a demand side perspective, multiple stakeholder engagement supports social inclusion in the health sector. Firstly, private sector contributions are recognized as pivotal to sustain health gains in some developing countries. Private health facilities are known to contribute substantially to health service delivery and transformation (Wolvardt \& Palmer, 1997). Furthermore, poor accountability in the health sector is attributed to the poor health sector coordination among multiple stakeholders. There is growing attention to general health demands to be improved to increase accountability at country and global levels on reproductive health (Chan et al., 2010). As mentioned in Chapter 4 of this dissertation, there is a need to train multiple skills of health professionals to effectively supervise and facilitate the quality of service provision. Also, working towards an effective health system that recognizes multiple stakeholder roles enhances health equity (Kelly et al., 2007). Secondly, public sector management and accountability is further enhanced when multiple stakeholders are engaged. The World Bank considers public sector management and accountability as critical components of governance systems (Cassels, 1995). Thus, the accountability and competence of a government are defined by public-private sector engagement that ensures social inclusion in health services delivery. Reproductive and maternity barriers are evidence of poor standards of social investments at community and facility levels. Removing such known barriers will require multiple interventions across several stakeholder level to improve efficiency and accountability (Chapter 4, 5 and 6).

\section{Research and policy implications}

We acknowledge the inability to include stakeholders at the health ministry level and other public-private partners involved in implementing reproductive health strategies in Ghana in our study. Future study should prioritize and triangulate these and many other stakeholders' roles that influence the provision, decision and policy making processes on reproductive care. 
As we examined in Chapter 6, to deliver accountable and equitable reproductive services provision, local health systems need to be resourced to evaluate health staff working attitudes. One critical aspect of this review relates to user needs and satisfied reproductive care demands. Policies that target private sector participation may result in more multi-faceted reproductive services provision, something not entirely available in most public sector health facilities. Stakeholder consensus from this study also strongly advocates clear guidelines on reproductive care to be contextualized to meet user care needs. Health counselling and the need for personalized care have increasingly been evidenced as an approach to reach new users of health services (Chapter 5). In furtherance of a more collective process in delivering reproductive and maternity care services in rural settings, we suggest the adoption of integrative participatory approaches in the delivery of reproductive health services, particularly along multiple stakeholders and among vulnerable population groups.

Statement 6: To improve reproductive health care, health services should evolve to incorporate patient-centered care values in their provision and utilization

As espoused in Chapter 1 of this dissertation, to reduce unmet needs for reproductive care, addressing patient-centered care needs remains important. One fundamental benefit to a patient-centered approach to care is the attainment of shared decision making by both health care staff and patients. This shared decision making enables health care staff to understand the values and preferences on reproductive care services of users. Aside these, patient centred approaches provide multiple routes along which patients feel part of the provision of reproductive care. Specifically, the approach offers an opportunity for health staff to better explore patients' main reasons for facility visits. It also addresses patient's integrated health needs. Ultimately, patient-centered interactions provide opportunities to realize shared information and attained aspirations among health staff and users. In committing to improve patient-centered care needs, health system challenges related to non-differentiation for reproductive care services, social stigma and infrastructure deficits need to be addressed. The reported limitations of reproductive care are indicative of the inadequacy of the diagnostic and treatment infrastructure in rural Ghana (Chapter 6). A health system that provides adequate skilled professional numbers may fail to attain patient-centered care if environmental conditions that guarantee the adequate provision of this care remain unmet.

As stated in Chapter 5 of this dissertation, women perceive that health staff provides "scanty" reproductive care information. This information does not address individual women needs on reproductive. Chapter 5 further shows that 
even among contraceptive users, women reiterated open censures suffered in the hands of health care staff when questioning them on alternative contraceptive methods. These actions personify unequal decision making roles, and little focus on the user's long term health consequences. Thus, in environments where such conditions exist, poor provider relations and covert reproductive services use become the norm (Chapter 5). Where reproductive services such as STIs, abortion/post-abortion care, and infertility treatments are limited in scope, adequately ensuring the three tiers of a patient-centered approach communication, partnership and health advocacy/promotion - are important to meet user reproductive expectations (Chapter 6).

Providing patient-centered, culturally sensitive and acceptable care that respects women's preferred clinic hours improves reproductive user satisfaction and subsequent future use (Chapter 5). Hence, patient-centered values should be able to meet user expectations on appropriate clinic hours among active users for reproductive care services. Our results in Chapter 6 show that reproductive counselling for rural women is often inadequately designed and not tailored towards individual needs. This often has the tendency to limit the positive impact counselling services would have on access to reproductive care services.

As presented in Chapter 6, delivering patient-centered reproductive health care services requires multiple stakeholder involvement and health systems that are more integrative. Aside this, local supportive health systems should exist and facilitate continuous provision of reproductive care services. We show that individualized reproductive care leading to patient-centered care would be difficult to attain when psychological support mechanisms are absent (Chapter 6). More so, to improve patient-centered reproductive care, emotional support to enable users to express their values and preferences without censures from health facility staff is important. Additionally, in recognizing the role played by multiple stakeholders in the continuum of reproductive care, health staff and users need to be treated as equal partners. Power role plays between health staff and reproductive users lower user levels among low educated, poor and rural Ghanaian women (Chapter 4, 5, and 6). To promote accountability and equity in reproductive services provision at facility levels, local health systems need to be resourced to evaluate patient-centered care practices. In addition, health staff attitudes impact on reproductive user needs and satisfied reproductive care demands. Recent advances in medical technologies may improve patient-centered care, especially among users with chronic reproductive health conditions. There is evidence that novel patient-centered health information technologies can improve patientcentered reproductive care. This is enhanced through the generation of timely diagnosis with a more efficient capacity to elicit user reproductive preferences (Bauman, Fardy, \& Harris, 2003; Little et al., 2001). Overall, health systems are 
inherently relational (Gilson, 2003). Thus, the extent to which health staff work attitudes and health care staff-provider power plays define access and quality of services delivered. There is need for broader provider-user engagements and social clinical friendlier support services to encourage women active participation in reproductive care seeking. To actively facilitate the delivery of patient-centered care, the active involvement of the right numbers of skilled professional numbers with the requisite skills set remains important.

\section{Research and policy implications}

Future research may examine in more detail the relationship between patientcentered care and individual health needs. In examining this it will be useful to examine the context amenable factors that promote or inhibit patient-centered care. Such findings may be helpful for strategy and policy formulation to improve the delivery of value based reproductive health care services.

To support the delivery of patient-centered care, policies that strive to upscale private sector services will increase private sector contributions for reproductive care. Improvements in reproductive counselling services must be user-specific and lead to the empowerment of the individual. Health system designs that allow for continuous feedback and psychosocial support among users further enhance reproductive decision making choices. Our findings advocate the inclusion and the effective dissemination of patient care delivery standards among various stakeholder groups. A culture of inclusiveness has the potential to promote trust and mutual respect in the provision of patient-centered care services. To ensure sustainable reproductive care needs, adequate financial planning to address informal payment complaints by women in meeting rural maternity care needs remains important.

\subsection{Final Remarks}

To meet women reproductive health service needs in rural Ghana, understanding contextual unmet demands on reproductive health care needs is important. Poor service quality and user satisfaction facilitate the existence of unmet reproductive health care. Hence, poor quality and low rated satisfied demands exacerbates reproductive unmet needs as illustrated by this dissertation. In addressing service quality and user's satisfaction needs on reproductive care, tailored policies by the Ministry of Health and Ghana Health Service should focus also on improving health system arrangements for reproductive care services delivery. Increased policies that ensure the availability of reproductive services among targeted adolescent groups are important. Focused counselling 
and context clinic arrangements are exemplified as important to rural Ghanaian women access and use of contraceptives. Non-governmental organizations involved in delivering interventions related to reproductive and maternity care reproductive services uptake should consider social and environmental needs in their programs.

Target driven and cost effective interventions that ensure services are delivered in socially accepted modes also enhance user's expectations for future services use. Health staff and policy implementers providing services need to understand social values, norms and beliefs systems in order to deliver acceptable services. Additionally, promoting the integration of reproductive health care services, and quality of consultation for reproductive health services will enhance focused maternity care in Ghana. Ongoing general services integration models need to be evaluated by the Ministry of Health to provide evidence of best integrated models on delivering reproductive services in Ghana. Prompt and adequate focused reproductive counselling assist health staff and user's shared decision making on reproductive care service use. Despite the progressive growth in subscriber numbers for health insurance in Ghana (out-patient utilization of healthcare services increased from 23.9 million in 2012 to 27.35 million in 2013) (NHIA, 2013), poor financial reimbursements affect health care access and quality in Ghana. A revision of the insurance type and benefits packages by the Ghana National Health Insurance Authority targeting pro-poor households and maternity care coverages will further improve womanhood initiatives. The current capitation reforms under the health insurance in Ghana should integrate value-based health care principles on care giving among vulnerable population groups.

Adequate ANC is not related to the frequency of visits made to the facility by a woman during pregnancy. Program interventions carried out by the Ghana Health Service and private sector health providers need to consider individual components of ANC services provided at facilities, rather than on once frequency of visits for ANC. Facility level staffs providing ANC services needs to be re-oriented on WHO focused components of ANC, emphasizing on quality indicators on ANC. This is because improving the coverage of focused ANC may address specific social and cultural barriers for ANC use among women. Adopting participatory approaches on reproductive services delivery is acknowledged by stakeholders as vital for improving reproductive care. In addition, stakeholder expectations show the need to improve health systems to become more integrative and supportive for delivering reproductive care in rural Ghana. Policies by the Ministry of Health on private sector participation need to be encouraged since this has potential to improve multi-faceted reproductive services provisions, something not entirely available in most public sector health facilities. 
Lastly, providing patient-centered and culturally sensitive care improves reproductive user satisfaction and future use. However, delivering patient-centered reproductive health care services requires multiple stakeholder involvement and health systems that are more integrative. Reproductive counselling services must be designed to be user-specific. In providing support for enhanced decision making, health system designs that allow for continuous feedback and psychosocial support among users, and improved professional skills to deliver the needed care is very important.

Our results advocate inclusive and effective dissemination of patient care delivery standards among stakeholder groups involved in the provision of care. A culture of inclusiveness has the potential to promote trust and mutual respect required in the provision of patient-centered care services. 


\section{References}

Abekah-Nkrumah, G., \& Abor, P. A. (2016). Socioeconomic determinants of use of reproductive health services in Ghana. Health economics review, 6(1), 1-15. doi: 10.1186/s13561-016-0085-7

Abou-Zahr, C. L. W., Tessa, M., Alfaro, A., Alvarado, N. M., Sánchez, R. G., Roaf, A. M., Priego, S. (2003). ANC in developing countries: promises, achievements and missed opportunities: an analysis of trends, levels and differentials, 1990-2001: Organización Mundial de la Salud.

AbouZahr, C., \& Wardlaw, T. (2001). Maternal mortality at the end of a decade: signs of progress? Bulletin of the World Health Organization, 79(6), 561-573.

Adanu, R. M. (2014). Utilization of obstetric services in Ghana between 1999 and 2003. African journal of reproductive health, 14(3), 153-158.

Adanu, R. M., Seffah, J. D., Hill, A. G., Darko, R., Duda, R. B., \& Anarfi, J. K. (2009). Contraceptive use by women in Accra, Ghana: results from the 2003 Accra Women's Health Survey. Afr J Reprod Health, 13(1), 123-133.

Addai, I. (1998). Demographic and sociocultural factors influencing use of maternal health services in Ghana. African journal of reproductive health, 2(1), 73-80.

Addai, I. (2000). Determinants of use of maternal-child health services in rural Ghana. Journal of Biosocial Science, 32(01), 1-15.

Adebayo, S. B., Gayawan, E., Ujuju, C., \& Ankomah, A. (2013). Modelling geographical variations and determinants of use of modern family planning methods among women of reproductive age in Nigeria. Journal of Biosocial Science, 45(01), 57-77.

Adeniran, A., Ogunnowo, B. E., Wright, K. O., \& Odusanya, O. O. (2015). Effect of Training on Knowledge and Practices of Traditional Birth Attendants for the Prevention of Malaria in Pregnancy in Nigeria. Journal of Public Health in Developing Countries, 1(2), 64-74.

Adetunji, J. A., \& Shelton, J. D. (2011). [Ethical Issues in the Collection, Analysis and Dissemination of DHS Data in sub-Saharan Africa.

Admassie, A., Abebaw, D., \& Woldemichael, A. D. (2009). Impact evaluation of the Ethiopian health services extension program. Journal of Development Effectiveness, 1(4), 430-449.

Adongo, P. B., Phillips, J. F., Kajihara, B., Fayorsey, C., Debpuur, C., \& Binka, F. N. (1997). Cultural factors constraining the introduction of family planning among the Kassena-Nankana of northern Ghana. Soc Sci Med, 45. doi: 10.1016/s0277-9536(97)00110-x

Agree, E. M., \& Freedman, V. A. (2003). A comparison of assistive technology and personal care in alleviating disability and unmet need. The Gerontologist, 43(3), 335-344.

Åhman, E., \& Shah, I. H. (2011). New estimates and trends regarding unsafe abortion mortality. International Journal of Gynecology \& Obstetrics, 115(2), 121-126.

Ajah, L. O., Dim, C. C., Ezegwui, H. U., Iyoke, C. A., \& Ugwu, E. O. (2015). Male partner involvement in female contraceptive choices in Nigeria. J Obstet Gynaecol, 35. doi: $10.3109 / 01443615.2014 .991287$

Alden, D. L., \& Bhawuk, D. (2004). Client satisfaction with reproductive health-care quality: integrating business approaches to modeling and measurement. Social science \& medicine, 59(11), 2219-2232. 
Alkema, L., Kantorova, V., Menozzi, C., \& Biddlecom, A. (2013). National, regional, and global rates and trends in contraceptive prevalence and unmet need for family planning between 1990 and 2015: a systematic and comprehensive analysis. The Lancet, 381(9878), 1642-1652.

Allen, M. (2011). Violence and voice: Using a feminist constructivist grounded theory to explore women's resistance to abuse. Qualitative Research, 11(1), 23-45.

APP. (2010). Maternal health: Investing in the lifeline of healthy societies and economies African Progress Panel Policy Brief.

Arthur, E. (2012). Wealth and ANC use: implications for maternal health care Utilization in Ghana. Health economics review, 2(1), 1-8.

Asamoah, B., Agardh, A., \& Ostergren, P. O. (2013). Inequality in fertility rate and modern contraceptive use among Ghanaian women from 1988-2008. International Journal for Equity in Health, 12(1), 37.

Asamoah, B. O., Agardh, A., Pettersson, K. O., \& Östergren, P. O. (2014). Magnitude and trends of inequalities in ANC and delivery under skilled care among different socio-demographic groups in Ghana from 1988-2008. BMC Pregnancy and childbirth, 14(1), 295.

Asante, F. A., Chikwama, C., Daniels, A., \& Armar-Klemesu, M. (2007). Evaluating the economic outcomes of the policy of fee exemption for maternal delivery care in Ghana. Ghana medical journal, 41(3).

Asekun-Olarinmoye, E., Adebimpe, W., Bamidele, J., Odu, O., Asekun-Olarinmoye, I., \& Ojofeitimi, E. (2013). Barriers to use of modern contraceptives among women in an inner city area of Osogbo metropolis, Osun state, Nigeria. International journal of women's health, 5, 647.

Asenso-Okyere, W., Anum, A., Osei-Akoto, I., \& Adukonu, A. (1998). Cost recovery in Ghana: Are there Any changes in health care seeking ? Health policy and planning, 13(2), 181-188.

Ashford, L. (2003). Unmet need for family planning: Recent trends and their implications for programs: Population Reference Bureau.

Atinga, R. A., \& Baku, A. A. (2013). Determinants of ANC quality in Ghana. International Journal of Social Economics, 40(10), 852-865.

Atkinson, P., Delamont, S., \& Hammersley, M. (1988). Qualitative research traditions: A British response to Jacob. Review of educational research, 58(2), 231-250.

Atuahene, M. D., Afari, E. O., Adjuik, M., \& Obed, S. (2016). Health knowledge, attitudes and practices of family planning service providers and clients in Akwapim North District of Ghana. Contraception and Reproductive Medicine, 1(1), 1.

Atunah-Jay, S. J., Pettingell, S., Ohene, S. A., Michael Oakes, J., \& Borowsky, I. W. (2013). The relationship between antenatal provider type and maternal care in rural Ghana: a cross-sectional study. Tropical Medicine \& International Health, 18(6), 678-686. doi: 10.1111/tmi.12098

Ayanore, M. A., \& Aryee, P. A. (2015). Determinants and Use of Family Planning Among Young Women (18-28 Years) Attending Health Facilities in Garu-Tempane District of Ghana. International Journal of Health Sciences, 3(4), 43-54.

Ayanore, M. A., Pavlova, M., \& Groot, W. (2016). Unmet reproductive health needs among women in some West African countries: a systematic review of outcome measures and determinants. Reproductive Health, 13(1), 5-5. 
Babalola, S., \& Fatusi, A. (2009). Determinants of use of maternal health services in Nigeria-looking beyond individual and household factors. BMC Pregnancy and childbirth, 9(1), 43.

Baiden, F., Amponsa-Achiano, K., Oduro, A., Mensah, T., Baiden, R., \& Hodgson, A. (2006). Unmet need for essential obstetric services in a rural district in northern Ghana: Complications of unsafe abortions remain a major cause of mortality. Public Health, 120(5), 421-426.

Baiden, F., Mensah, G. P., Akoto, N. O., Delvaux, T., \& Appiah, P. C. (2016). Covert contraceptive use among women attending a reproductive health clinic in a municipality in Ghana. BMC Women's Health, 16(1), 1-10. doi: 10.1186/s12905-016-0310-x

Baidoo, R. (2009). Toward a comprehensive healthcare system in Ghana. Ohio University.

Baker, D. W., Sudano, J. J., Albert, J. M., Borawski, E. A., \& Dor, A. (2001). Lack of health insurance and decline in overall health in late middle age. New England Journal of Medicine, 345(15), 1106-1112.

Banchani, E., \& Tenkorang, E. Y. (2014). Implementation challenges of maternal health care in Ghana: the case of health care providers in the Tamale Metropolis. BMC Health Services Research, 14(1), 1-10. doi: 10.1186/1472-6963-14-7

Bankole, A., \& Ezeh, A. C. (1999). Unmet need for couples: an analytical framework and evaluation with DHS data. Population Research and Policy Review, 18(6), 579-605.

Baschieri, A., Cleland, J., Floyd, S., Dube, A., Msona, A., \& Molesworth, A. (2013). Reproductive preferences and contraceptive use: A comparison of monogamous and polygamous couples in northern Malawi. J Biosoc Sci, 45. doi: 10.1017/s0021932012000569

Bauman, A. E., Fardy, H. J., \& Harris, P. G. (2003). Getting it right: why bother with patient-centered care? Medical Journal of Australia, 179(5), 253-256.

Bawah, A. A., Akweongo, P., Simmons, R., \& Phillips, J. F. (1999). Women's fears and men's anxieties: the impact of family planning on gender relations in northern Ghana. Stud Fam Plann, 30. doi: 10.1111/j.1728-4465.1999.00054.x

Beegle, K., Frankenberg, E., \& Thomas, D. (2001). Bargaining power within couples and use of prenatal and delivery care in Indonesia. Studies in family planning, 32(2), 130-146.

Benach, J., Muntaner, C., Santana, V., \& Chairs, F. (2007). Employment conditions and health inequalities. Final report to the WHO Commission on Social Determinants of Health (CSDH) Employment Conditions Knowledge Network (EMCONET). Geneva.

Biddlecom, A. E., Munthali, A., Singh, S., \& Woog, V. (2007). Adolescents' views of and preferences for sexual and reproductive health services in Burkina Faso, Ghana, Malawi and Uganda. African journal of reproductive health, 11(3), 99.

Binka, F. N., Nazzar, A., \& Phillips, J. F. (1995). The Navrongo Community Health and Family Planning Project. Stud Fam Plann, 26. doi: 10.2307/2137832

Black, B. S., Rabins, P. V., German, P., McCuire, M., \& Roca, R. (1997). Need and unmet need for mental health care among elderly public housing residents. The Gerontologist, 37(6), 717-728.

Blanc, A. K. (2001a). The effect of power in sexual relationships on sexual and reproductive health: an examination of the evidence. Studies in family planning, 32(3), 189-213.

Blanc, A. K. (2001b). The effect of power in sexual relationships on sexual and reproductive health: an examination of the evidence. Stud Fam Plann, 32. doi: 10.1111/j.1728-4465.2001.00189.x 
Blanc, A. K., \& Grey, S. (2002). Greater than expected fertility decline in Ghana: untangling a puzzle. Journal of Biosocial Science, 34(04), 475-495.

Blanchet, N. J., Fink, G., \& Osei-Akoto, I. (2012). The effect of Ghana's National Health Insurance Scheme on health care Utilization. Ghana medical journal, 46(2), 76-84.

Blas, E., Gilson, L., Kelly, M. P., Labonté, R., Lapitan, J., Muntaner, C., Sen, G. (2008). Addressing social determinants of health inequities: what can the state and civil society do? The Lancet, 372(9650), 1684-1689.

Bloom, D. E., Sachs, J. D., Collier, P., \& Udry, C. (1998). Geography, demography, and economic growth in Africa Vol. Vol. 1998, No. 2 (1998). Brookings chapter s on economic activity (pp. 207-295). Retrieved from http://www.jstor.org/sTable/2534695 doi:10.2307/2534695

Bongaarts, J. (1991). The KAP-gap and the unmet need for contraception. Population and development review, 293-313.

Borghi, J., Ensor, T., Somanathan, A., Lissner, C., Mills, A., \& group, L. M. S. S. s. (2006). Mobilising financial resources for maternal health. The Lancet, 368(9545), 1457-1465.

Borghi, J., Hanson, K., Acquah, C. A., Ekanmian, G., Filippi, V., Ronsmans, C., Alihonou, E. (2003). Costs of near-miss obstetric complications for women and their families in Benin and Ghana. Health policy and planning, 18(4), 383-390.

Bove, R. M., Vala-Haynes, E., \& Valeggia, C. R. (2012a). Women's health in urban Mali: Social predictors and health itineraries. Social science \& medicine, 75(8), 1392-1399.

Bove, R. M., Vala-Haynes, E., \& Valeggia, C. R. (2012b). Women's health in urban Mali: Social predictors and health itineraries. Journal of Social science \& medicine, 75(8), 1392-1399.

Bradley, S. E., \& Casterline, J. B. (2014). Understanding unmet need: history, theory, and measurement. Studies in family planning, 45(2), 123-150.

Brazier, E., Andrzejewski, C., Perkins, M. E., Themmen, E. M., Knight, R. J., \& Bassane, B. (2009). Improving poor women's access to maternity care: Findings from a primary care intervention in Burkina Faso. Social science \& medicine, 69(5), 682-690.

Bremner, J., Frost, A., Haub, C., Mather, M., Ringheim, K., \& Zuehlke, E. (2010). World population highlights: key findings from PRB's 2010 world population data sheet. Population Reference Bureau.

Buchan, J., \& Calman, L. (2004). The global shortage of registered nurses: An overview of issues and actions. ICN, Geneva.

Buor, D. (2003). Analysing the primacy of distance in the utilization of health services in the AhafoAno South district, Ghana. The International Journal of Health Planning and Management, 18(4), 293-311.

Campbell, M., Sahin-Hodoglugil, N. N., \& Potts, M. (2006). Barriers to Fertility Regulation: A Review of the Literature. Stud Fam Plann, 37(2), 87-98. doi: 10.1111/j.1728-4465.2006.00088.x

Cassels, A. (1995). Health sector reform: key issues in less developed countries. Journal of international development, 7(3), 329-347.

Casterline, J. B., \& Sinding, S. W. (2000). Unmet need for family planning in developing countries and implications for population policy. Population and development review, 26(4), 691-723. 
Chan, M., Kazatchkine, M., Lob-Levyt, J., Obaid, T., Schweizer, J., Sidibe, M., Yamada, T. (2010). Meeting the demand for results and accountability: a call for action on health data from eight global health agencies. PLoS Med, 7(1), e1000223.

Charmaz, K. (2006). Constructing grounded theory: A practical guide through qualitative research. SagePublications Ltd, London.

Chigbu, C. O., Onyebuchi, A. K., Onwudiwe, E. N., \& Iwuji, S. E. (2013). Denial of women's rights to contraception in southeastern Nigeria. International Journal of Gynecology \& Obstetrics, 121(2), 154-156.

Cleland, J., Bernstein, S., Ezeh, A., Faundes, A., Glasier, A., \& Innis, J. (2006a). Family planning: the unfinished agenda. The Lancet, 368(9549), 1810-1827.

Cleland, J., Bernstein, S., Ezeh, A., Faundes, A., Glasier, A., \& Innis, J. (2006b). Family planning: the unfinished agenda. The Lancet, 368. doi: 10.1016/s0140-6736(06)69480-4

Cleland, J., \& Shah, I. H. (2013). The contraceptive revolution: focused efforts are still needed. The Lancet, 381(9878), 1604-1606.

Colaizzi, P. F. (1978). Psychological research as the phenomenologist views it.

Corroon, M., Speizer, I. S., Fotso, J.-C., Akiode, A., Saad, A., Calhoun, L., \& Irani, L. (2014). The role of gender empowerment on reproductive health outcomes in urban Nigeria. Maternal and child health journal, 18(1), 307-315.

Crissman, H. P., Adanu, R. M., \& Harlow, S. D. (2012). Women's Sexual Empowerment and Contraceptive Use in Ghana. Studies in family planning, 43(3), 201-212. doi: 10.1111/j.17284465.2012.00318.x

Crissman, H. P., Engmann, C. E., Adanu, R. M., Nimako, D., Crespo, K., \& Moyer, C. A. (2013). Shifting norms: pregnant women's perspectives on skilled birth attendance and facility-based delivery in rural Ghana. African journal of reproductive health, 17(1), 15-26.

d'Ambruoso, L., Abbey, M., \& Hussein, J. (2005). Please understand when I cry out in pain: women's accounts of maternity services during labour and delivery in Ghana. BMC Public Health, 5(1), 140.

Dalaba, M. A., Stone, A. E., Krumholz, A. R., Oduro, A. R., Phillips, J. F., \& Adongo, P. B. (2016). A qualitative analysis of the effect of a community-based primary health care program on reproductive preferences and contraceptive use among the Kassena-Nankana of northern Ghana. BMC Health Services Research, 16(1), 1-8. doi: 10.1186/s12913-016-1325-6

Darroch, J. E. (2013). Trends in contraceptive need and use in developing countries in 2003, 2008, and 2012: an analysis of national surveys. Contraception, 87(3), 259-263.

Darroch, J. E., \& Singh, S. (2011). Estimating unintended pregnancies averted by couple-years of protection (CYP). New York, 1-10.

Darroch, J. E., \& Singh, S. (2013). Trends in contraceptive need and use in developing countries in 2003, 2008, and 2012: An Analysis of National Surveys. The Lancet, 381(9879), 1756-1762.

De Allegri, M., Ridde, V., Louis, V. R., Sarker, M., Tiendrebéogo, J., Yé, M., Jahn, A. (2011). Determinants of Utilization of maternal care services after the reduction of user fees: a case study from rural Burkina Faso. Health Policy, 99(3), 210-218. 
Debpuur, C., Phillips, J. F., Jackson, E. F., Nazzar, A., Ngom, P., \& Binka, F. N. (2002). The impact of the Navrongo Project on contraceptive knowledge and use, reproductive preferences, and fertility. Studies in family planning, 33(2), 141-164.

Dehne, K., \& Snow, R. (1999). Integrating STI management into family planning services: what are the benefits?

DeJong, J., Shepard, B., Roudi-Fahimi, F., \& Ashford, L. (2007). Young people's sexual and reproductive health in the Middle East and North Africa. Reproductive Health, 14(78), 8.

DeRose, L. F., Dodoo, F. N.-A., Ezeh, A. C., \& Owuor, T. O. (2004). Does discussion of family planning improve knowledge of partner's attitude toward contraceptives? Int Fam Plan Perspect, 87-93.

DeRose, L. F., Dodoo, F. N.-A., \& Patil, V. (2002). Fertility desires and perceptions of power in reproductive conflict in Ghana. Gender \& Society, 16(1), 53-73.

Devasenapathy, N., George, M. S., Jerath, S. G., Singh, A., Negandhi, H., Alagh, G., Zodpey, S. (2014). Why women choose to give birth at home: a situational analysis from urban slums of Delhi. BMJ open, 4(5), e004401.

Diamant, A. L., Hays, R. D., Morales, L. S., Ford, W., Calmes, D., Asch, S., Fielding, J. (2004). Delays and unmet need for health care among adult primary care patients in a restructured urban public health system. American Journal of Public Health, 94(5), 783-789.

Dixon-Mueller, R., \& Germain, A. (1992). Stalking the elusive" unmet need” for family planning. Studies in family planning, 23(5), 330-335.

Dixon, J., Tenkorang, E. Y., Luginaah, I. N., Kuuire, V. Z., \& Boateng, G. O. (2014). National health insurance scheme enrolment and ANC among women in Ghana: is there any relationship? Tropical Medicine \& International Health, 19(1), 98-106.

Do, M., \& Kurimoto, N. (2012). Women's empowerment and choice of contraceptive methods in selected African countries. International perspectives on sexual and reproductive health, 23-33.

Do, M., Soelaeman, R., \& Hotchkiss, D. R. (2014). Explaining Inequity in the Use of Institutional Delivery Services in Selected Countries. Maternal and child health journal, 1-9.

Dogba, M., Fournier, P., Dumont, A., Zunzunegui, M.-V., Tourigny, C., \& Berthe-Cisse, S. (2011). Mother and newborn survival according to point of entry and type of human resources in a maternal referral system in Kayes (Mali). Reprod Health, 8(1), 13.

Domar, A. D. (2004). Impact of psychological factors on dropout rates in insured infertility patients. Fertility and sterility, 81(2), 271-273.

Doyle, A. M., Mavedzenge, S. N., Plummer, M. L., \& Ross, D. A. (2012). The sexual of adolescents in sub-Saharan Africa: patterns and trends from national surveys. Tropical Medicine \& International Health, 17(7), 796-807.

Dudgeon, M. R., \& Inhorn, M. C. (2004). Men's influences on women's reproductive health: medical anthropological perspectives. Social science \& medicine, 59(7), 1379-1395.

Eliason, S., Baiden, F., Quansah-Asare, G., Graham-Hayfron, Y., Bonsu, D., Phillips, J., \& AwusaboAsare, K. (2013a). Factors influencing the intention of women in rural Ghana to adopt postpartum family planning. Reproductive Health, 10(1), 34 . 
Eliason, S., Baiden, F., Quansah-Asare, G., Graham-Hayfron, Y., Bonsu, D., Phillips, J., \& AwusaboAsare, K. (2013). Factors influencing the intention of women in rural Ghana to adopt postpartum family planning. Reprod Health, 10. doi: 10.1186/1742-4755-10-34

Eliason, S., Baiden, F., Quansah-Asare, G., Graham-Hayfron, Y., Bonsu, D., Phillips, J., \& AwusaboAsare, K. (2013). Factors influencing the intention of women in rural Ghana to adopt postpartum family planning. Reprod Health, 10(1), 34 .

Ensor, T., \& Ronoh, J. (2005). Effective financing of maternal health services: a review of the literature. Health Policy, 75(1), 49-58.

Erim, D. O., Resch, S. C., \& Goldie, S. J. (2012). Assessing health and economic outcomes of interventions to reduce pregnancy-related mortality in Nigeria. BMC Public Health, 12(1), 786.

Farmer, P., Frenk, J., Knaul, F. M., Shulman, L. N., Alleyne, G., Armstrong, L., Feachem, R. (2010). Expansion of cancer care and control in countries of low and middle income: a call to action. The Lancet, 376(9747), 1186-1193.

Filippi, V., Ronsmans, C., Campbell, O. M., Graham, W. J., Mills, A., Borghi, J., Osrin, D. (2006). Maternal health in poor countries: the broader context and a call for action. The Lancet, 368(9546), 1535-1541.

Fotso, J. C., Ezeh, A. C., \& Essendi, H. (2009). Maternal health in resource-poor urban settings: how does women's autonomy influence the utilization of obstetric care services. Reprod Health, 6(9), 1-8.

Frimpong, P. B. (2013). The Quest for Equity in the Provision of health Care in Ghana. African Review of Economics and Finance, 4(2), 254-272.

Furuta, M., \& Salway, S. (2006). Women's position within the household as a determinant of maternal health care use in Nepal. International Family Planning Perspectives, 17-27.

Gakidou, E., \& Vayena, E. (2007). Use of modern contraception by the poor is falling behind. PLoS Med, 4(2), e31.

Ganle, J. K. (2014). Addressing socio-cultural barriers to maternal healthcare in Ghana: Perspectives of women and healthcare providers. J Womens Health, Issues Care 3, 6, 2.

Ganle, J. K., Parker, M., Fitpatrick, R., \& Otupiri, E. (2015). Addressing Health System Barriers to Access to and Use of skilled delivery services: Perspectives from Ghana. Int J Health Plann Manag.

Gayawan, E. (2013). A Poisson Regression Model to Examine Spatial Patterns in ANC Utilization in Nigeria. Population, Space and Place.

Gerein, N., Green, A., \& Pearson, S. (2006). The implications of shortages of health professionals for maternal health in sub-Saharan Africa. Reproductive Health Matters, 14(27), 40-50.

GHS. (2009). The Health Sector In Ghana; Facts and Figures, 2002-2008. Accra, Ghana.

Gilson, L. (2003). Trust and the development of health care as a social institution. Social science \& medicine, 56(7), 1453-1468.

Glaser, B. G. (1965). The constant comparative method of qualitative analysis. Social problems, 12(4), 436-445. 
Glasier, A., Gülmezoglu, A. M., Schmid, G. P., Moreno, C. G., \& Van Look, P. F. (2006). Sexual and reproductive health: a matter of life and death. The Lancet, 368(9547), 1595-1607.

GOG. (2000). Ghana Adolescent Reproductive Health Policy. Accra, Ghana: National Population Council.

Grimes, D. A., Benson, J., Singh, S., Romero, M., Ganatra, B., Okonofua, F. E., \& Shah, I. H. (2006). Unsafe abortion: the prevenTable pandemic. The Lancet, 368(9550), 1908-1919. doi: http://dx.doi. org/10.1016/S0140-6736(06)69481-6

GSS. (2012). 2010 Population and Housing Census Final Report. Accra,Ghana.

GSS, GHS, \& ICF. (2014). Ghana Demographic and Health Survey. Accra, Ghana.

GSS, GHS, \& ICF, M. (2009). Ghana Demopgraphic and Health Survey 2008. Accra Ghana: GSS, GHS, and ICF Macro.

Habicht, J., Xu, K., Couffinhal, A., \& Kutzin, J. (2006). Detecting changes in financial protection: creating evidence for policy in Estonia. Health policy and planning, 21(6), 421-431.

Hall, K. S., Manu, A., Morhe, E., Challa, S., Loll, D., Dozier, J., Adanu, R. (2016). Stigma and the Social Environment of Adolescent Sexual and Reproductive Health in Ghana: Understanding "Bad Girl". Journal of Adolescent Health, 58(2), S4.

Hanschmidt, F., Linde, K., Hilbert, A., Riedel-Heller, S. G., \& Kersting, A. (2016). Abortion Stigma: A Systematic Review. Perspectives on sexual and reproductive health.

Hauser, P. M. (1983). The limitations of KAP Surveys. Social research in developing countries: Surveys and censuses in the third world.

Higgins, J. P., \& Green, S. (2008). Cochrane handbook for systematic reviews of interventions (Vol. 5): Wiley Online Library.

Hill, Z. E., Tawiah-Agyemang, C., \& Kirkwood, B. (2009). The context of informal abortions in rural Ghana. Journal of Women's Health, 18(12), 2017-2022.

Horev, T., \& Babad, Y. M. (2005). Healthcare reform implementation: stakeholders and their roles-the Israeli experience. Health Policy, 71(1), 1-21. doi: http://dx.doi.org/10.1016/j. healthpol.2004.05.001

Izugbara, C., Ibisomi, L., Ezeh, A. C., \& Mandara, M. (2010). Gendered interests and poor spousal contraceptive communication in Islamic northern Nigeria. Journal of Family Planning and Reproductive Health Care, 36(4), 219-224.

Izugbara, C. O., \& Ezeh, A. C. (2010). Women and high fertility in Islamic northern Nigeria. Studies in family planning, 41(3), 193-204.

Jacobstein, R., Curtis, C., Spieler, J., \& Radloff, S. (2013). Meeting the need for modern contraception: Effective solutions to a pressing global challenge. International Journal of Gynecology \& Obstetrics, 121, S9-S15.

Jamal, V., \& Weeks, J. (1988). Vanishing Rural-Urban Gap in Sub-Saharan Africa, The. Int'l Lab. Rev., 127, 271.

Jewkes, R., Abrahams, N., \& Mvo, Z. (1998). Why do nurses abuse patients? Reflections from South African obstetric services. Social Science \& Medicine, 47(11), 1781-1795. 
Jimenez, V., Klein, M. C., Hivon, M., \& Mason, C. (2010). A Mirage of Change: Family-Centered Maternity Care in Practice. Birth, 37(2), 160-167.

Johnson, F. A., \& Madise, N. J. (2011). Targeting women at risk of unintended pregnancy in Ghana: Should geography matter? Sexual \& Reproductive Healthcare, 2(1), 29-35.

Joseph Kofi, T. (2013). Modern Contraceptive Use among Women in the Asuogyaman District of Ghana: Is Reliability More Important than Health Concerns? Afr J Reprod Health, 17(2), 58.

Kelly, M.P., Morgan, A., Bonnefoy, J., Butt, J., Bergman, V. (2007). The social determinants of health developing an evidence base for political action. Final Report to World Health Organization Commission on the Social Determinants of Health. NHS.

Kiapi-Iwa, L., \& Hart, G. J. (2004). The sexual and reproductive health of young people in Adjumani district, Uganda: qualitative study of the role of formal, informal and traditional health providers. AIDS care, 16(3), 339-347.

Kirk, D., \& Pillet, B. (1998). Fertility levels, trends, and differentials in sub-Saharan Africa in the 1980s and 1990s. Studies in family planning, Vol. 29, No. 1 (Mar., 1998), 1-22. doi: 10.2307/172178

Kish, L. (1986). Timing of surveys for public policy. Australian Journal of Statistics, 28(1), 1-12.

Koblinsky, M., Tain, F., Gaym, A., Karim, A., Carnell, M., \& Tesfaye, S. (2010). Responding to the maternal health care challenge: The Ethiopian Health Extension Program. Ethiopian Journal of Health Development, 24(1).

Kodzi, I. A., Johnson, D. R., \& Casterline, J. B. (2012). To have or not to have another child: Life cycle, health and cost considerations of Ghanaian women. Social Science \& Medicine, 74(7), 966-972.

Kruk, M. E., Rockers, P. C., Mbaruku, G., Paczkowski, M. M., \& Galea, S. (2010). Community and health system factors associated with facility delivery in rural Tanzania: a multilevel analysis. Health Policy, 97(2), 209-216.

Lambon-Quayefio, M. P., \& Owoo, N. S. (2014). Examining the Influence of ANC Visits and Skilled Delivery on Neonatal Deaths in Ghana. Applied health economics and health policy, 12(5), 511-522.

LaPlante, M. P., Kaye, H. S., Kang, T., \& Harrington, C. (2004). Unmet need for personal assistance services: Estimating the shortfall in hours of help and adverse consequences. The Journals of Gerontology Series B: Psychological Sciences and Social Sciences, 59(2), S98-S108.

Larson, E., Hermosilla, S., Kimweri, A., Mbaruku, G. M., \& Kruk, M. E. (2014). Determinants of perceived quality of obstetric care in rural Tanzania: a cross-sectional study. BMC Health Services Research, 14(1), 483.

Lincetto, O., Mothebesoane-Anoh, S., Gomez, P., \& Munjanja, S. (2006). ANC. Opportunities for Africa's newborns: Practical data, policy and programmatic support for newborn care in Africa.

Little, P., Everitt, H., Williamson, I., Warner, G., Moore, M., Gould, C., Payne, S. (2001). Preferences of patients for patient centred approach to consultation in primary care: observational study. Bmj, 322(7284), 468.

Loaiza, E., \& Blake, S. (2010). How universal is access to reproductive health? A review of the evidence. (334113). from United Nations Population Fund [UNFPA] http://www.popline.org/ node $/ 552290$ 
Lohr, K. N., \& Schroeder, S. A. (1990). A strategy for quality assurance in Medicare. New England Journal of Medicine, 322(10), 707-712.

Lori, J. R., Rominski, S. D., Gyakobo, M., Muriu, E. W., Kweku, N. E., \& Agyei-Baffour, P. (2012). Perceived barriers and motivating factors influencing student midwives' acceptance of rural postings in Ghana. Hum Resour Health, 10, 17.

Luxford, K., Safran, D. G., \& Delbanco, T. (2011). Promoting patient-centered care: a qualitative study of facilitators and barriers in healthcare organizations with a reputation for improving the patient experience. International Journal for Quality in Health Care, 23(5), 510-515.

Madi, B. C., Hussein, J., Hounton, S., D’Ambruoso, L., Achadi, E., \& Arhinful, D. K. (2007). Setting priorities for safe motherhood program evaluation: A participatory process in three developing countries. Health Policy, 83(1), 94-104. doi: http://dx.doi.org/10.1016/j.healthpol.2007.01.006

Magadi, M. A., Madise, N. J., \& Rodrigues, R. N. (2000). Frequency and timing of ANC in Kenya: explaining the variations between women of different communities. Social science \& medicine, 51(4), 551-561. doi: http://dx.doi.org/10.1016/S0277-9536(99)00495-5

Mahapatro, S. (2012). Utilization of maternal and child health care services in India: does women's autonomy matter. The Journal of Family Welfare, 58(1), 22-33.

Maharaj, P., \& Cleland, J. (2005). Integration of sexual and reproductive health services in KwaZuluNatal, South Africa. Health policy and planning, 20(5), 310-318.

Mahler, K. (1999). Covert method use among urban Zambian women is tied to poor spousal communication on contraception. Int Fam Plan Perspect, 25. doi: 10.2307/2991949

Malhotra, A., \& Mehra, R. (1999). Fulfilling the Cairo Commitment: Enhancing women's economic and social options for better reproductive health: International Center for Research on Women.

Marmot, M., Friel, S., Bell, R., Houweling, T. A., Taylor, S. (2008). Commission on the Social Determinants of Health. Closing the gap in a generation: health equity through action on the social determinants of health. The Lancet, 372(9650), 1661-1669.

Mathole, T., Lindmark, G., Majoko, F., \& Ahlberg, B. M. (2004). A qualitative study of women's perspectives of ANC in a rural area of Zimbabwe. Midwifery, 20(2), 122-132.

Mayhew, S. H., \& Adjei, S. (2004). Sexual and reproductive health: challenges for priority-setting in Ghana's health reforms. Health policy and planning, 19(suppl 1), i50-i61.

McIntosh, C. A., \& Finkle, J. L. (1995). The Cairo Conference on Population and Development: A New Paradigm? Population and development review, 21(2), 223-260. doi: 10.2307/2137493

McPake, B., Brikci, N., Cometto, G., Schmidt, A., \& Araujo, E. (2011). Removing user fees: learning from international experience to support the process. Health policy and planning, 26(suppl 2), ii104-ii117.

Medhanyie, A., Spigt, M., Kifle, Y., Schaay, N., Sanders, D., Blanco, R., Berhane, Y. (2012). The role of health extension workers in improving utilization of maternal health services in rural areas in Ethiopia: a cross sectional study. BMC Health Services Research, 12(1), 1.

Meij, J., De Craen, A., Agana, J., Plug, D., \& Westendorp, R. (2009). Low-cost interventions accelerate epidemiological transition in Upper East Ghana. Transactions of the Royal Society of Tropical Medicine and Hygiene, 103(2), 173-178. 
Metcalfe, Q. M. R. (2010). Understanding women's empowerment and maternal mortality in the Ugandan context: effects of mitigative intervention strategies. (M.A. M.A.), Simon Fraser University.

Miller, W. B., \& Pasta, D. J. (1995). Behavioral intentions: which ones predict fertility Behavior in married couples? 1. Journal of Applied Social Psychology, 25(6), 530-555.

Mills, S., Bos, E., \& Emi, S. (2010). Unmet need for contraception: World Bank.

MOH. (2007). Creating wealth through health. Accra,Ghana.

MOH. (2008). National consultative meeting on the reduction of maternal mortality in Ghana: partnership for action - a synthesis report. Accra.

MOH. (2008). National Consultative Meeting on the Reduction of Maternal Mortality in Ghana: Partnership for Action, Ministry of Health, A synthesis Report. Accra, Ghana.

MOH. (2011). Independent Review Health Sector Program of Work 2010. Accra, Ghana.

MOH. (2014). Holistic Assessment of the Health Sector Program of Work 2013. Ministry of Health, Accra, Ghana.

Mosha, I., Ruben, R., \& Kakoko, D. (2013). Family planning decisions, perceptions and gender dynamics among couples in Mwanza, Tanzania: a qualitative study. BMC public health, 13(1), 523.

Moyer, C. A., Adongo, P. B., Aborigo, R. A., Hodgson, A., \& Engmann, C. M. (2014). 'They treat you like you are not a human being': maltreatment during labour and delivery in rural northern Ghana. Midwifery, 30(2), 262-268.

Moyer, C. A., Adongo, P. B., Aborigo, R. A., Hodgson, A., Engmann, C. M., \& DeVries, R. (2014). "It's up to the woman's people": how social factors influence facility-based delivery in Rural Northern Ghana. Maternal and child health journal, 18(1), 109-119.

Mumtaz, Z., \& Salway, S. (2005). 'I never go anywhere': extricating the links between women's mobility and uptake of reproductive health services in Pakistan. Social science \& medicine, 60(8), 1751-1765.

Mutemwa, R., Mayhew, S., Colombini, M., Busza, J., Kivunaga, J., \& Ndwiga, C. (2013). Experiences of health care providers with integrated HIV and reproductive health services in Kenya: a qualitative study. BMC Health Services Research, 13(1), 18. doi: 10.1186/1472-6963-13-18.

Newacheck, P. W., Hughes, D. C., Hung, Y.-Y., Wong, S., \& Stoddard, J. J. (2000). The unmet health needs of America's children. Pediatrics, 105(Supplement 3), 989-997.

NHIA (2013). 2013 Annual Report (pp. 13). Accra, Ghana.

Nikiema, B., Haddad, S., \& Potvin, L. (2012). Measuring women's perceived ability to overcome barriers to healthcare seeking in Burkina Faso. BMC Public Health, 12(1), 147.

Odeyemi, I., \& Nixon, J. (2013). Assessing equity in health care through the national health insurance schemes of Nigeria and Ghana: a review-based comparative analysis. International Journal for Equity in Health, 12(1), 9. doi: 10.1186/1475-9276-12-9

Ogu, R., Okonofua, F., Hammed, A., Okpokunu, E., Mairiga, A., Bako, A., Agholor, K. (2012). Outcome of an intervention to improve the quality of private sector provision of postabortion care in northern Nigeria. International Journal of Gynecology \& Obstetrics, 118, S121-S126. 
Okafor, I., Obi, S., \& Ugwu, E. (2010). Impact of Free Maternal and Child Healthcare program on maternal and neonatal healthcare outcome in Enugu State of Nigeria. Nigerian Journal of Medicine, 20(4), 441-443.

Okoli, U., Abdullahi, M. J., Pate, M. A., Abubakar, I. S., Aniebue, N., \& West, C. (2012). Prenatal care and basic emergency obstetric care services provided at primary healthcare facilities in rural Nigeria. International Journal of Gynecology \& Obstetrics, 117(1), 61-65.

OlaOlorun, F., Seme, A., Otupiri, E., Ogunjuyigbe, P., \& Tsui, A. (2016). Women's fertility desires and contraceptive behavior in three peri-urban communities in sub Saharan Africa. Reproductive Health, 13(1), 1-6. doi: 10.1186/s12978-016-0118-z

Olayemi, O., Bello, F., Aimakhu, C., Obajimi, G., \& Adekunle, A. (2009). Male participation in pregnancy and delivery in Nigeria: a survey of antenatal attendees. Journal of biosocial science, 41(04), 493-503.

Olugbenga-Bello, A., Abodunrin, O., \& Adeomi, A. (2011). Contraceptive practices among women in rural communities in south-western Nigeria. Global Journal of Medical Research, Vol. 11, no. 2 (2011).

Omeje, J. C., Oshi, S. N., \& Oshi, D. C. (2011). Does possession of assets increase women's participation in reproductive decision-making? Perceptions of nigerian women. Journal of biosocial science, 43(01), 101-111.

Ononokpono, D. N., Odimegwu, C. O., Imasiku, E., \& Adedini, S. (2013). Contextual determinants of maternal health care service utilization in Nigeria. Women \& health, 53(7), 647-668.

Opare, M., \& Mill, J. E. (2000). The evolution of nursing education in a postindependence contextGhana from 1957 to 1970 . Western journal of nursing research, 22(8), 936-944.

Opit, L. J. (1987). How should information on health care be generated and used? Chapter presented at the World Health Forum.

Overbosch, G., Nsowah-Nuamah, N., van den Boom, G., \& Damnyag, L. (2004). Determinants of ANC use in Ghana. Journal of African Economies, 13(2), 277-301.

Oyerinde, K., Harding, Y., Amara, P., Garbrah-Aidoo, N., Kanu, R., Oulare, M., Daoh, K. (2013). A qualitative evaluation of the choice of traditional birth attendants for maternity care in 2008 Sierra Leone: implications for universal skilled attendance at delivery. Maternal and child health journal, 17(5), 862-868.

Parr, N. J. (2003). Discontinuation of contraceptive use in Ghana. Journal of Health, Population and Nutrition, 150-157.

Patterson, K. D. (1981). Health in Colonial Ghana: disease, medicine, and socio-economic change, 1900-1955: Epstein Building, Brandeis University, Waltham, Massachusetts 02254, USA; Crossroads Press.

Payne, C. M., Debbink, M. P., Steele, E. A., Buck, C. T., Martin, L. A., Hassinger, J. A., \& Harris, L. H. (2013). Why women are dying from unsafe abortion: narratives of Ghanaian abortion providers. African journal of reproductive health, 17(2), 118-128.

Pell, C., Meñaca, A., Were, F., Afrah, N. A., Chatio, S., Manda-Taylor, L., . . Kalilani, L. (2013). Factors affecting ANC attendance: results from qualitative studies in Ghana, Kenya and Malawi. PloS one, 8(1), e53747. 
Perkins, M., Brazier, E., Themmen, E., Bassane, B., Diallo, D., Mutunga, A., Ngobola, O. (2009). Out-of-pocket costs for facility-based maternity care in three African countries. Health policy and planning, 24(4), 289-300.

Phillips, J. F., Bawah, A. A., \& Binka, F. N. (2006). Accelerating reproductive and child health program impact with community-based services: the Navrongo experiment in Ghana. Bulletin of the World Health Organization, 84(12), 949-955.

Requejo, J. H., Bryce, J., Barros, A. J., Berman, P., Bhutta, Z., Chopra, M., Maliqi, B. (2015). Countdown to 2015 and beyond: fulfilling the health agenda for women and children. The Lancet, 385(9966), 466-476.

Ridde, V., \& Morestin, F. (2011). A scoping review of the literature on the abolition of user fees in health care services in Africa. Health policy and planning, 26(1), 1-11.

Rifkin, S. B. (1986). Health planning and community participation. Chapter presented at the World Health Forum.

Ross, J., \& Hardee, K. (2013). Access to contraceptive methods and prevalence of use. Journal of Biosocial Science, 45(06), 761-778.

Ross, J. A., \& Winfrey, W. L. (2001). Contraceptive use, intention to use and unmet need during the extended postpartum period. International Family Planning Perspectives, 20-27.

Rottach, E., Schuler, S. R., \& Hardee, K. (2009). Gender perspectives improve reproductive health outcomes: new evidence.

Rudan, I., Kapiriri, L., Tomlinson, M., Balliet, M., Cohen, B., \& Chopra, M. (2010). Evidence-based priority setting for health care and research: tools to support policy in maternal, neonatal, and child health in Africa. PLOS medicine, 7(7), 824.

Say, L., \& Raine, R. (2007). A systematic review of inequalities in the use of maternal health care in developing countries: examining the scale of the problem and the importance of context. Bulletin of the World Health Organization, 85(10), 812-819.

Scheil-Adlung, X., Carrin, G., Juetting, J., \& Xu, K. (2006). What is the impact of social health protection on access to health care, health expenditure and impoverishment? A comparative analysis of three African countries.

Seddoh, A., \& Akor, S. A. (2012). Policy initiation and political levers in health policy: lessons from Ghana's health insurance. BMC Public Health, 12(1), 1.

Sedgh, G., \& Hussain, R. (2014). Reasons for contraceptive nonuse among women having unmet need for contraception in developing countries. Studies in family planning, 45(2), 151-169.

Sedgh, G., Hussain, R., Bankole, A., \& Singh, S. (2007). Women with an Unmet need for contraception in developing countries and the reason for not using a method Occasional reprot No.37 Guttmacher Institute.

Sedgh, G., Hussain, R., Bankole, A., \& Singh, S. (2007). Women with an unmet need for contraception in developing countries and their reasons for not using a method: Citeseer.

Sedgh, G., Singh, S., Shah, I. H., Åhman, E., Henshaw, S. K., \& Bankole, A. (2012). Induced abortion: incidence and trends worldwide from 1995 to 2008. The Lancet, 379(9816), 625-632. 
Senah, K. (2001). In sickness and in health: globalization and health care delivery in Ghana. [http:// www.ajol.info/index.php/rrias/article/view/22902]. Research Review of the Institute of African Studies, 17, 83-89.

Sialubanje, C., Massar, K., Hamer, D. H., \& Ruiter, R. A. (2015). Reasons for home delivery and use of traditional birth attendants in rural Zambia: a qualitative study. BMC Pregnancy and childbirth, 15(1), 216.

Simkhada, B., Teijlingen, E. R. v., Porter, M., \& Simkhada, P. (2008). Factors affecting the utilization of ANC in developing countries: systematic review of the literature. Journal of Advanced Nursing, 61(3), 244-260.

Simkin, P. (1991). Just another day in a woman's life? Women's long-term perceptions of their first birth experience. Part I. Birth, 18(4), 203-210.

Singh, S., \& Darroch, J. E. (2012). Adding it up: Costs and benefits of contraceptive services. Guttmacher Institute and UNFPA.

Singh, S., \& Darroch, J. E. (2012). Adding it up: costs and benefits of contraceptive servicesestimates for 2012. Guttmacher Institute and United Nations Population Fund (UNFPA), New York.

Singh, S., Darroch, J. E., Vlassoff, M., Nadeau, J., \& UNFPA (2003). Adding it up: the benefits of investing in sexual and reproductive health care: Alan Guttmacher Institute, New York.

Sitzia, J., \& Wood, N. (1997). Patient satisfaction: a review of issues and concepts. Social science \& medicine, 45(12), 1829-1843.

Slade, P., O'Neill, C., Simpson, A. J., \& Lashen, H. (2007). The relationship between perceived stigma, disclosure patterns, support and distress in new attendees at an infertility clinic. Human Reproduction, 22(8), 2309-2317.

Smith, M. E., Tawiah, E. O., \& Badasu, D. M. (2012). Why some women deliver in health institutions and others do not: A cross sectional study of married women in Ghana, 2008. Afr J Reprod Health, 16(3).

Smith, R., Ashford, L., Gribble, J., \& Clifton, D. (2009). Family planning saves lives.

Somé, D. T., Sombié, I., \& Meda, N. (2013). How decision for seeking maternal care is made-a qualitative study in two rural medical districts of Burkina Faso. Reprod Health, 10(8), 47554710.

Speizer, I. S., Calhoun, L. M., Hoke, T., \& Sengupta, R. (2013). Measurement of unmet need for family planning: longitudinal analysis of the impact of fertility desires on subsequent childbearing behaviors among urban women from Uttar Pradesh, India. Contraception, 88(4), 553-560.

Srivastava, A., Avan, B. I., Rajbangshi, P., \& Bhattacharyya, S. (2015). Determinants of women's satisfaction with maternal health care: a review of literature from developing countries. BMC Pregnancy \& Childbirth, 15(1), 1.

Stephenson, R., Baschieri, A., Clements, S., Hennink, M., \& Madise, N. (2007). Contextual influences on modern contraceptive use in sub-Saharan Africa. American journal of public health, 97(7), 1233.

Tautz, S., Jahn, A., Molokomme, I., \& Görgen, R. (2000). Between fear and relief: how rural pregnant women experience foetal ultrasound in a Botswana district hospital. Social science \& medicine, 50(5), 689-701. 
ten Hoope-Bender, P., de Bernis, L., Campbell, J., Downe, S., Fauveau, V., Fogstad, H., McFadden, A. (2014). Improvement of maternal and newborn health through midwifery. The Lancet, 384(9949), 1226-1235.

Tsui, A. O., McDonald-Mosley, R., \& Burke, A. E. (2010). Family planning and the burden of unintended pregnancies. Epidemiologic reviews, mxq012.

Turinawe, E. B., Rwemisisi, J. T., Musinguzi, L. K., de Groot, M., Muhangi, D., de Vries, D. H., Pool, R. (2016). Traditional birth attendants (TBAs) as potential agents in promoting male involvement in maternity preparedness: insights from a rural community in Uganda. Reproductive Health, 13(1), 1 .

Twumasi, P. A. (1981). Colonialism and international health: a study in social change in Ghana. Social Science \& Medicine. Part B: Medical Anthropology, 15(2), 147-151.

UNFPA. (1999). Safe motherhood: evaluation report, New York.

UNICEF, \& WHO. (2012). Trends in maternal mortality: 1990 to 2010. UNFPA and The World Bank estimates, Geneva.

Van de Walle, E. (1992). Fertility transition, conscious choice, and numeracy. demography, 29(4), 487-502.

van der Geugten, J., Dijkstra, M., van Meijel, B., den Uyl, M. H. G., \& de Vries, N. K. (2015). Sexual and reproductive health education: opinions of students and educators in Bolgatanga municipality, northern Ghana. Sex Education, 15(2), 113-128. doi: 10.1080/14681811.2014.968771

Verma, J. (2013). Cluster Analysis: For Segmenting the Population Data Analysis in Management with SPSS Software (pp. 317-358): Springer.

Vlassoff, M., Walker, D., Shearer, J., Newlands, D., \& Singh, S. (2009). Estimates of health care system costs of unsafe abortion in Africa and Latin America. International perspectives on sexual and reproductive health, 114-121.

Von Both, C., Fleßa, S., Makuwani, A., Mpembeni, R., \& Jahn, A. (2006). How much time do health services spend on ANC? Implications for the introduction of the focused ANC model in Tanzania. BMC Pregnancy and childbirth, 6(1), 22. doi: 10.1186/1471-2393-6-22.

Waddington, C., \& Enyimayew, K. (1990). A price to pay, part 2: The impact of user charges in the Volta region of Ghana. The International Journal of Health Planning and Management, 5(4), 287-312.

Walsh, D. (1999). An ethnographic study of women's experience of partnership caseload midwifery practice: the professional as a friend. Midwifery, 15(3), 165-176.

Watkins, K. (2014). Leaving no one behind: an agenda for equity. The Lancet, 384(9961), 2248-2255.

World Bank (2012). Ghana country data. from http://data.worldbank.org/country/ghana, accessed on 31st March, 2016.

World Bank (2013). Ghana country data. from http://data.worldbank.org/country/ghana, accessed on 31st March, 2016.

Wells, K., Klap, R., Koike, A., \& Sherbourne, C. (2001). Ethnic disparities in unmet need for alcoholism, drug abuse, and mental health care. American Journal of Psychiatry. 
Welsh, E. (2002). Dealing with data: Using NVivo in the qualitative data analysis process. Chapter presented at the Forum Qualitative Sozialforschung/Forum: Qualitative Social Research.

Westoff, C. F., \& Bankole, A. (1996). The potential demographic significance of unmet need. International Family Planning Perspectives, 16-20.

Westoff, C. F., \& Ochoa, L. H. (1991). Unmet need and the demand for family planning.

Westoff, C. F., \& Pebley, A. R. (1981). Alternative measures of unmet need for family planning in developing countries. International Family Planning Perspectives, 7(4), 126-136.

White, D., Dynes, M., Rubardt, M., Sissoko, K., \& Stephenson, R. (2013). The influence of intrafamilial power on maternal health care in Mali: perspectives of women, men and mothersin-law. International perspectives on sexual and reproductive health, 58-68.

WHO. (2002). WHO ANC randomized trial: manual for the implementation of the new model. Geneva: World Health Organization, 37.

WHO. (2010). The World Health Report:health systems financing:the path to universal health coverage Geneva,Switzerland: World Health Organization.

WHO. (2011). WHO guidelines on preventing early pregnancy and poor reproductive health outcomes among adolescents in developing countries.

WHO. (2012). Addressing the Challenges of Women's Health in Africa Regional Office for Africa. Brazzaville: WHO Regional Office for Africa.

WHO. (2014). WHO recommendations on postnatal care of the mother and newborn: World Health Organization.

WHO. (2015). Sexual health, human rights and the law: World Health Organization.

Withers, M., Dworkin, S., Harrington, E., Kwena, Z., Onono, M., Bukusi, E., Newmann, S. J. (2013). Fertility intentions among HIV-infected, sero-concordant couples in Nyanza province, Kenya. Cult Health Sex, 15. doi: 10.1080/13691058.2013.811289

Witter, S., \& Adjei, S. (2007). Start-stop funding, its causes and consequences: a case study of the delivery exemptions policy in Ghana. The International Journal of Health Planning and Management, 22(2), 133-143.

Witter, S., Adjei, S., Armar-Klemesu, M., \& Graham, W. (2009). Providing free maternal health care: ten lessons from an evaluation of the national delivery exemption policy in Ghana. Global Health Action, 2.

Witter, S., Arhinful, D., Kusi, A., \& Zakariah-Akoto, S. (2007). The experience of Ghana in implementing a user fee exemption policy to provide free delivery care. Reprod Health Matters, 15. doi: 10.1016/s0968-8080(07)30325-x

Witter, S., Arhinful, D. K., Kusi, A., \& Zakariah-Akoto, S. (2007). The experience of Ghana in implementing a user fee exemption policy to provide free delivery care. Reproductive Health Matters, 15(30), 61-71.

Witter, S., Garshong, B., \& Ridde, V. (2013a). An exploratory study of the policy process and early implementation of the free NHIS coverage for pregnant women in Ghana. International Journal for Equity in Health, 12(1), 1-11. doi: 10.1186/1475-9276-12-16. 
Witter, S., Garshong, B., \& Ridde, V. (2013b). An exploratory study of the policy process and early implementation of the free NHIS coverage for pregnant women in Ghana. Int J Equity Health, 12(16), 1-11.

Wolvardt, G., \& Palmer, N. (1997). Private sector. South African Health Review, 1997, 37-44.

Yakong, V. N., Rush, K. L., Bassett-Smith, J., Bottorff, J. L., \& Robinson, C. (2010). Women's experiences of seeking reproductive health care in rural Ghana: challenges for maternal health service utilization. Journal of Advanced Nursing, 66(11), 2431-2441. doi: 10.1111/j.13652648.2010.05404.x

Zabin, L. S., \& Kiragu, K. (1998). The health consequences of adolescent sexual and fertility behavior in sub-Saharan Africa. Studies in family planning, 210-232.

Zere, E., Kirigia, J. M., Duale, S., \& Akazili, J. (2012). Inequities in maternal and child health outcomes and interventions in Ghana. BMC public health, 12(1), 252. 



\section{Appendix A \\ Overview of publications assessed and presented in Chapter 2}




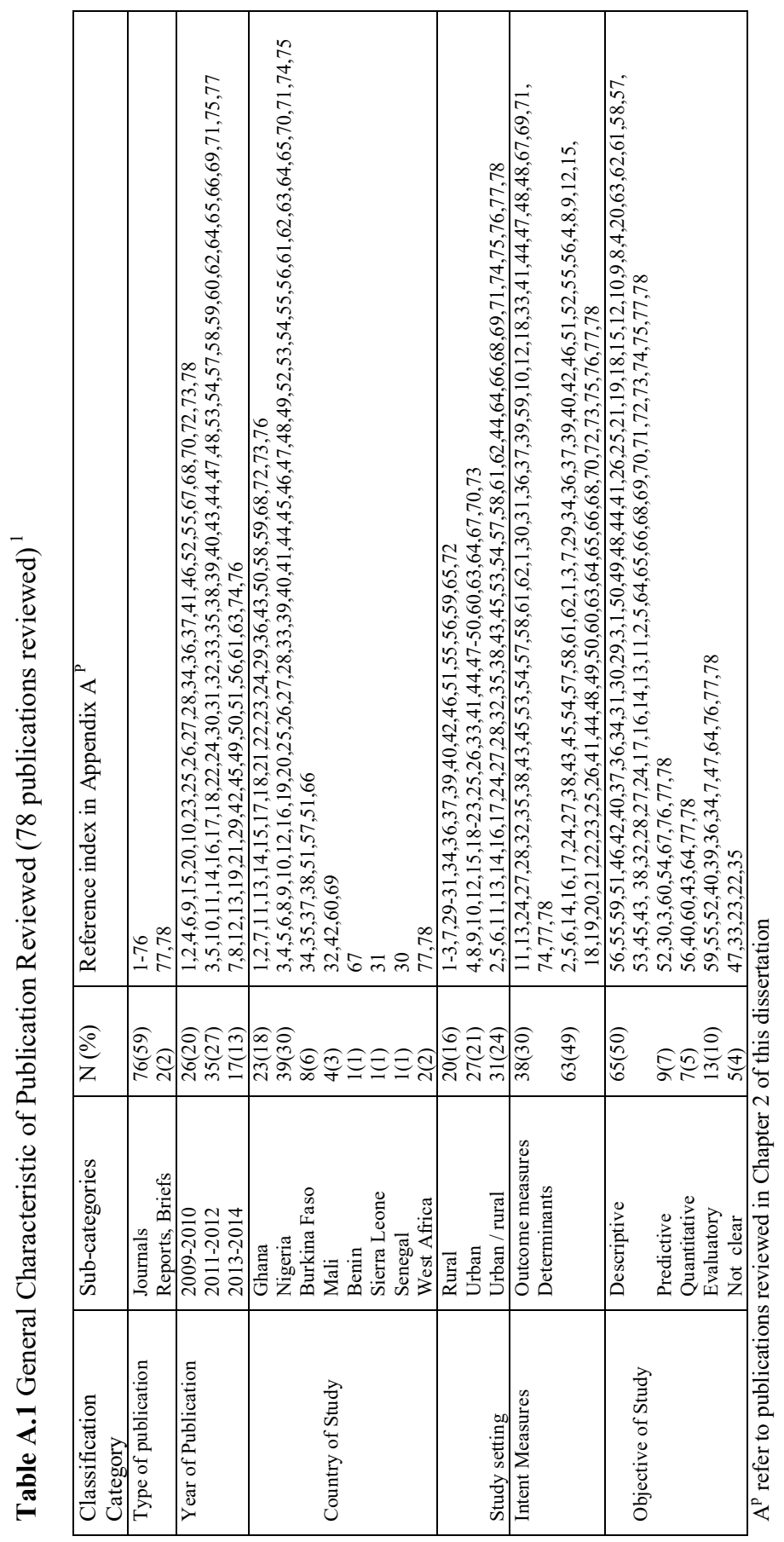




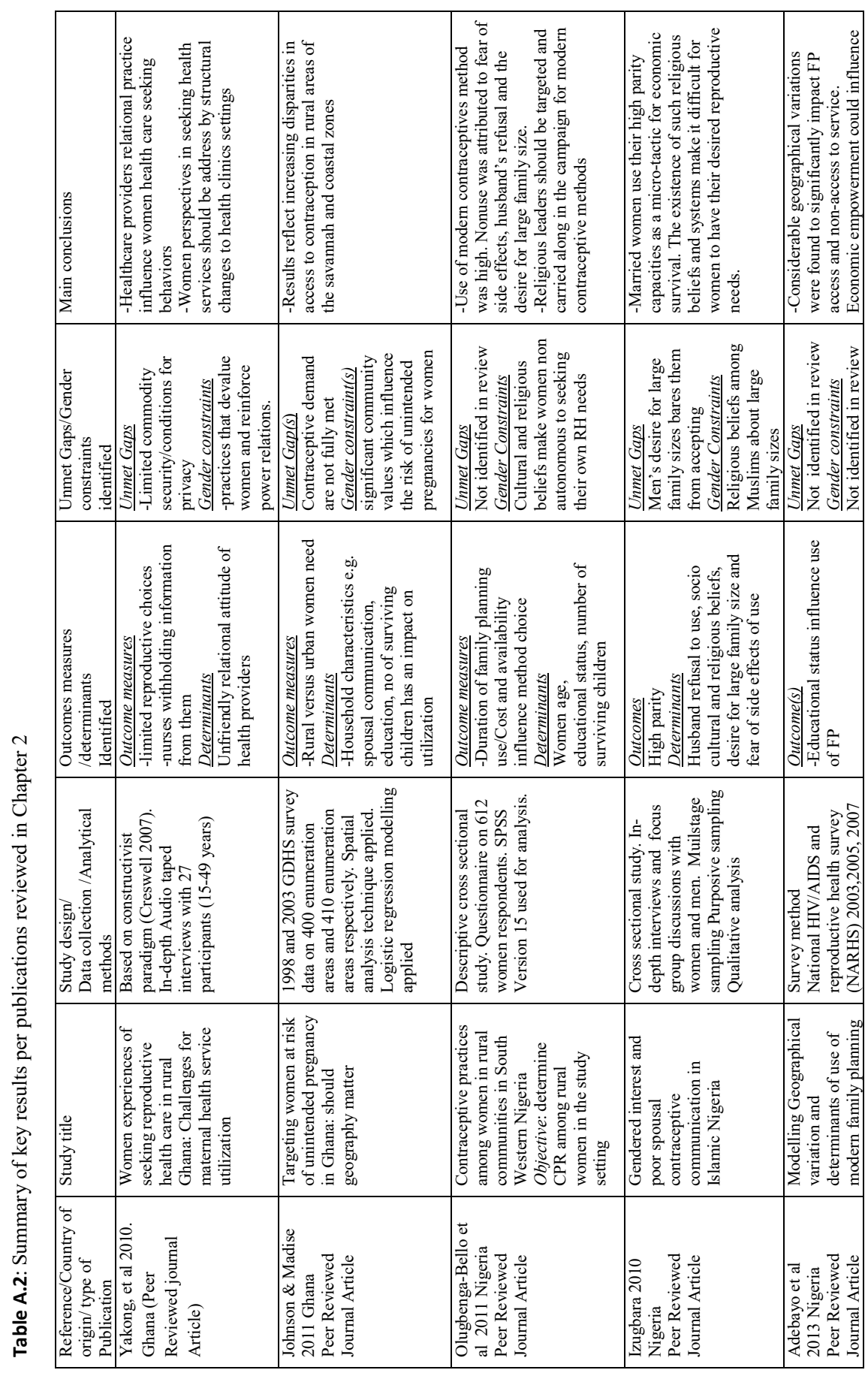




\begin{tabular}{|c|c|c|c|c|}
\hline 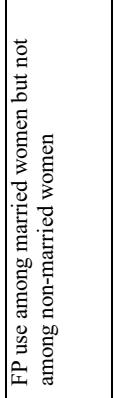 & 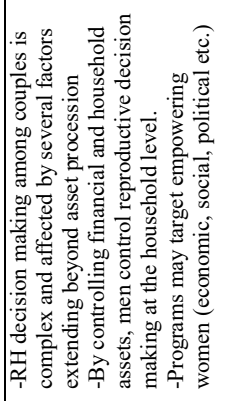 & 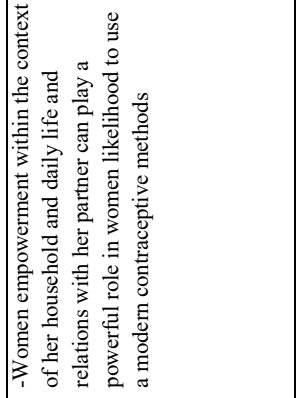 & 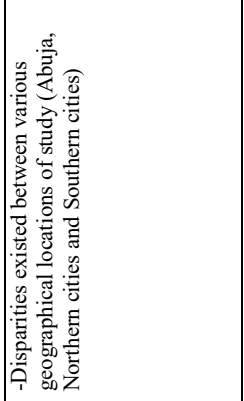 & 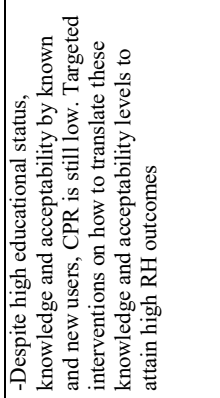 \\
\hline & 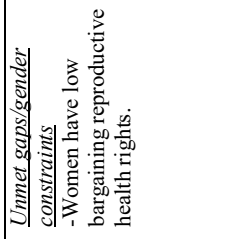 & 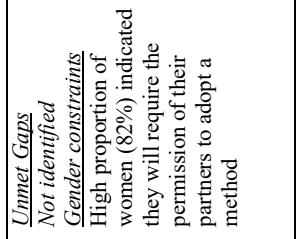 & 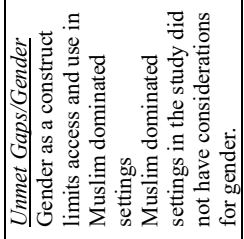 & 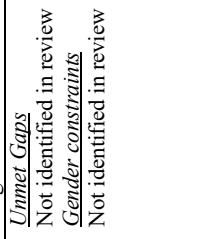 \\
\hline 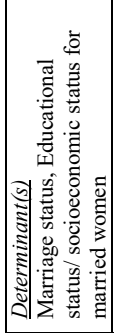 & 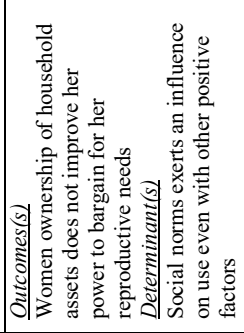 & 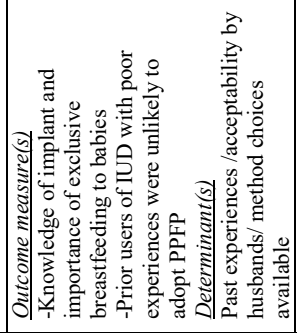 & 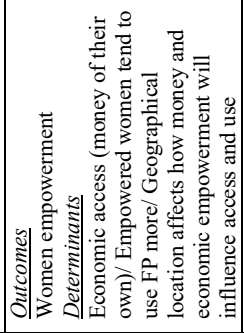 & 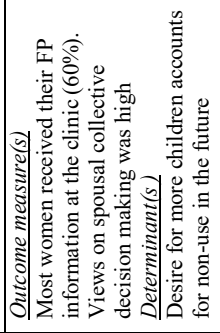 \\
\hline 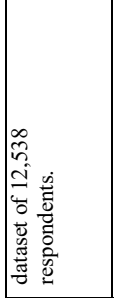 & 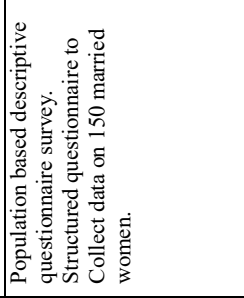 & 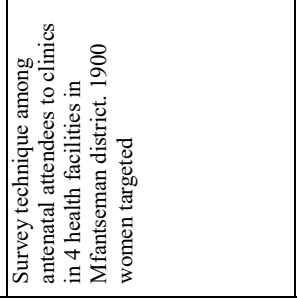 & 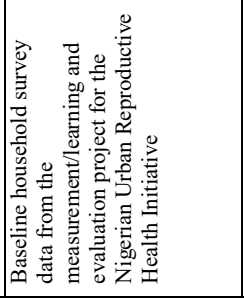 & 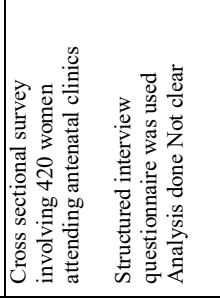 \\
\hline \multirow[t]{2}{*}{ 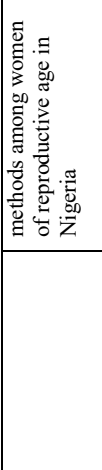 } & 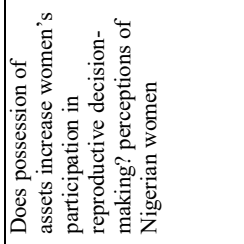 & 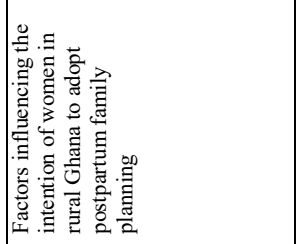 & 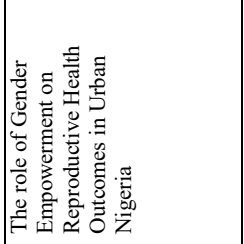 & 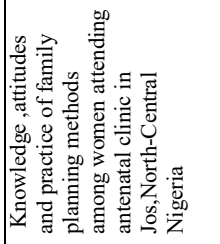 \\
\hline & 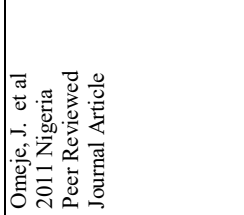 & 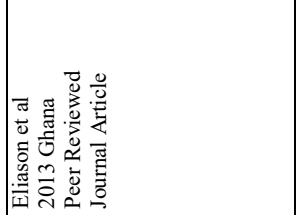 & 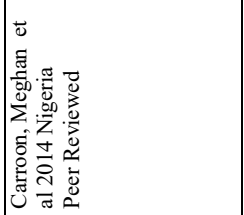 & 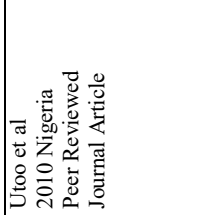 \\
\hline
\end{tabular}




\begin{tabular}{|c|c|c|c|c|c|}
\hline 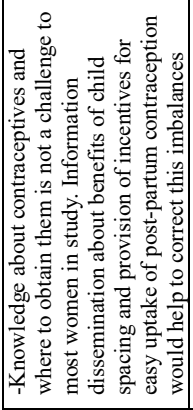 & 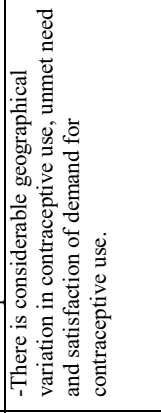 & 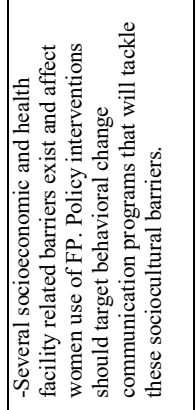 & 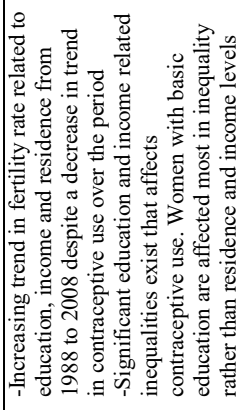 & 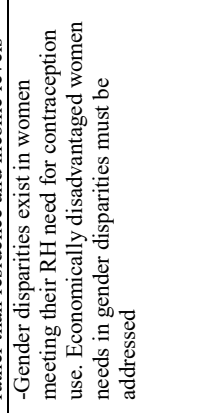 & 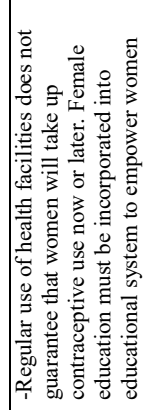 \\
\hline 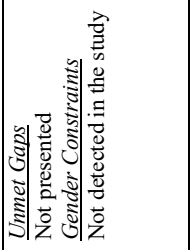 & 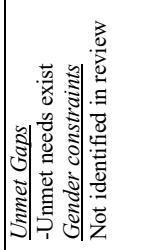 & 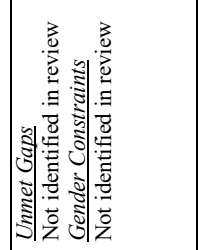 & 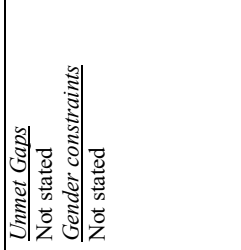 & 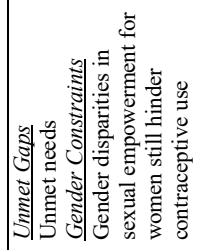 & | \\
\hline 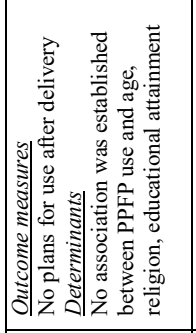 & 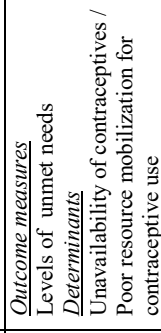 & 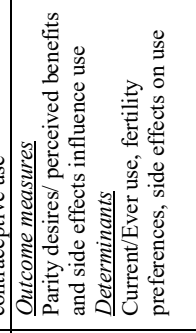 & 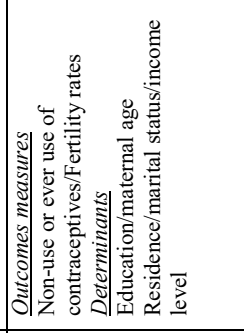 & 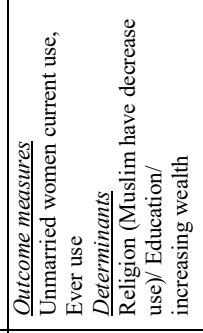 & 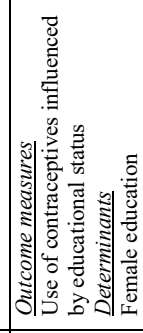 \\
\hline 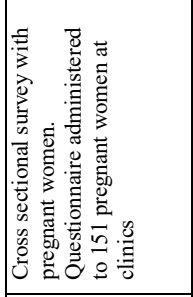 & 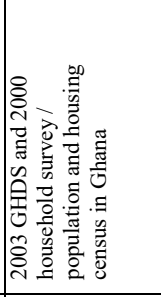 & 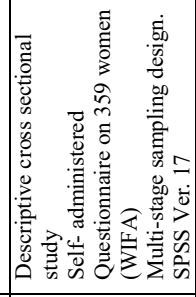 & 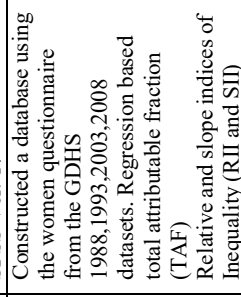 & 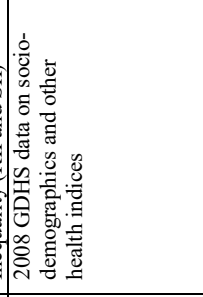 & 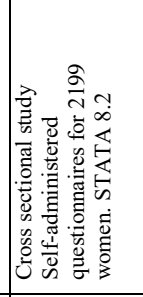 \\
\hline 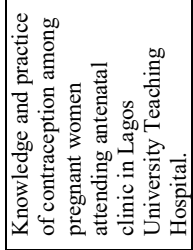 & 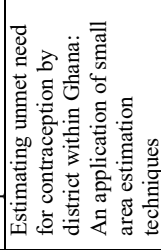 & 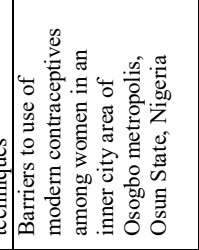 & 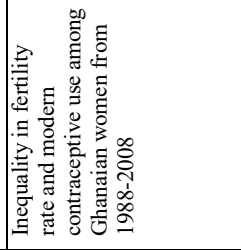 & 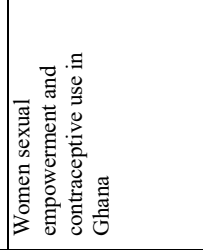 & 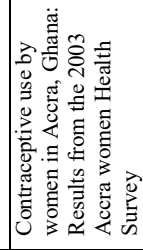 \\
\hline 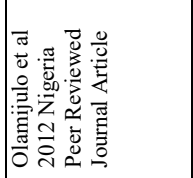 & 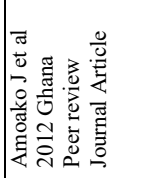 & 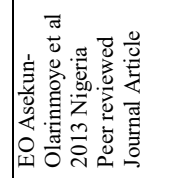 & 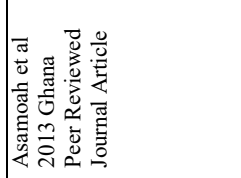 & 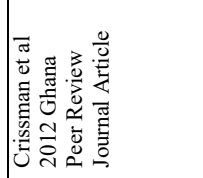 & 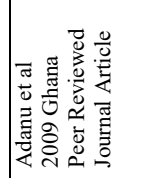 \\
\hline
\end{tabular}




\begin{tabular}{|c|c|c|c|c|c|}
\hline 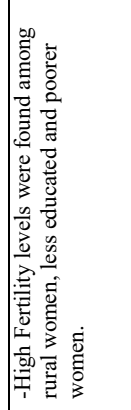 & 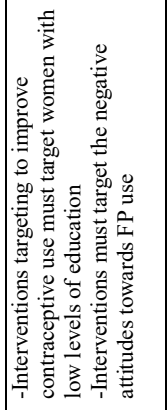 & 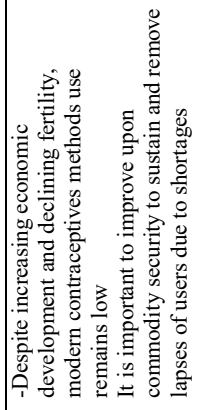 & 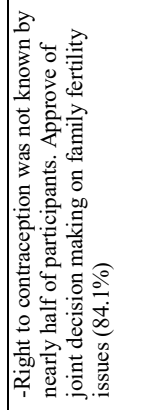 & 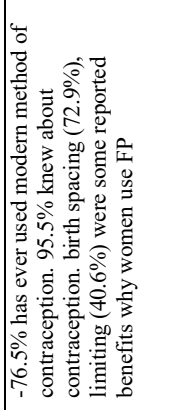 & 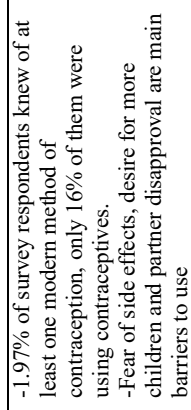 \\
\hline 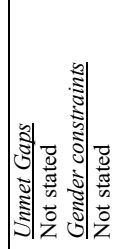 & 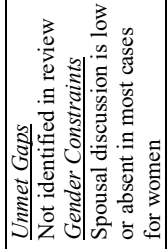 & 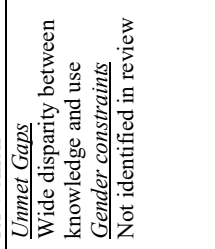 & 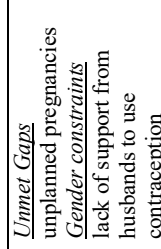 & 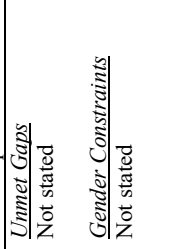 & 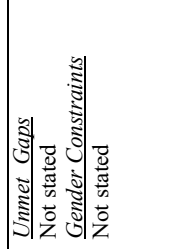 \\
\hline 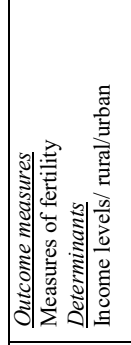 & 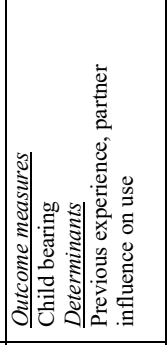 & 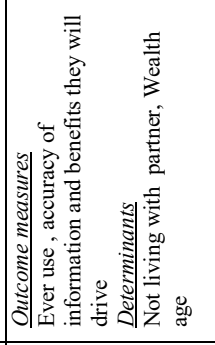 & 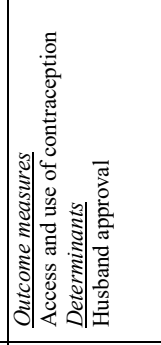 & 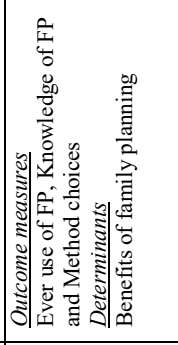 & 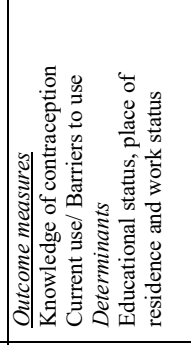 \\
\hline 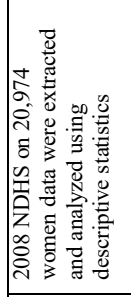 & 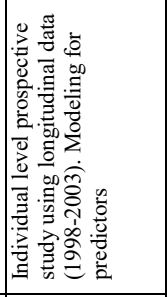 & 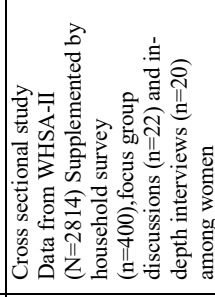 & 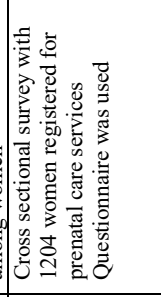 & 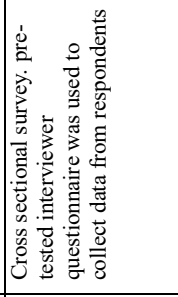 & 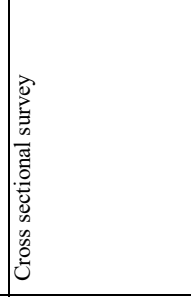 \\
\hline 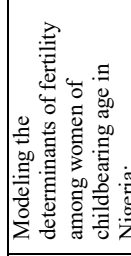 & 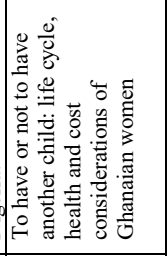 & 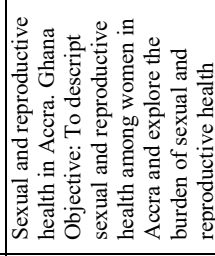 & 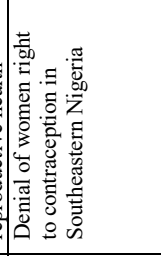 & 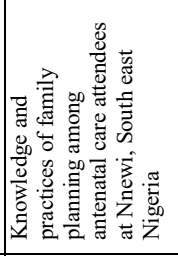 & 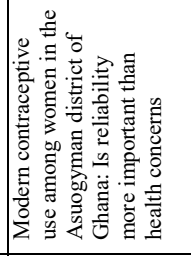 \\
\hline 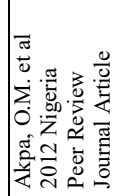 & 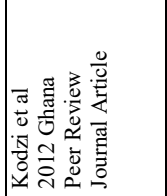 & 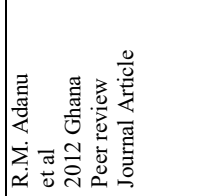 & 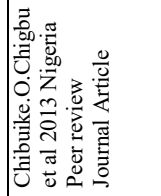 & 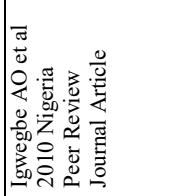 & 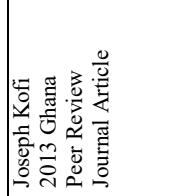 \\
\hline
\end{tabular}




\begin{tabular}{|c|c|c|c|c|c|c|}
\hline 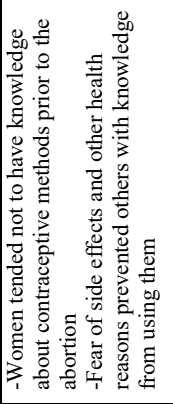 & 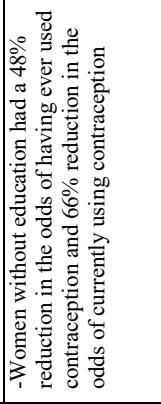 & 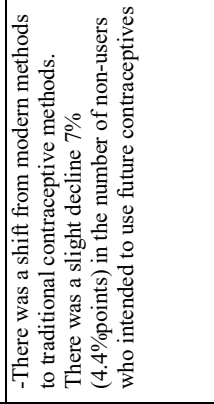 & 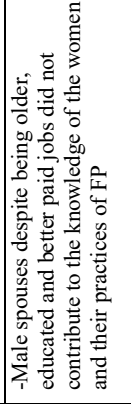 & 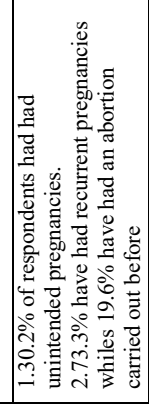 & 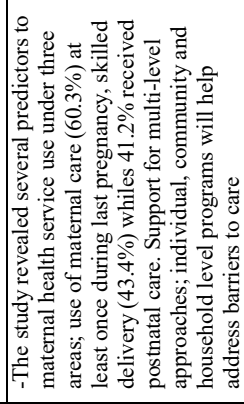 & 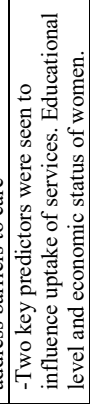 \\
\hline 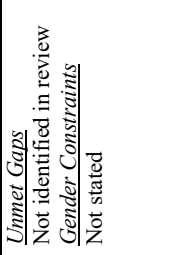 & 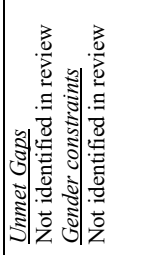 & 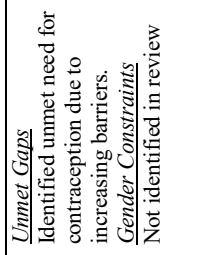 & 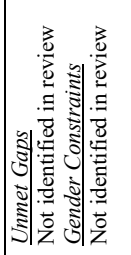 & 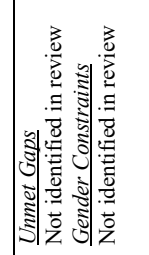 & 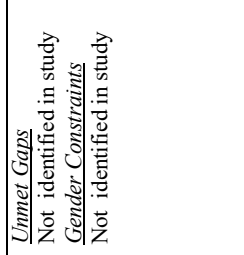 & 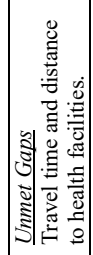 \\
\hline 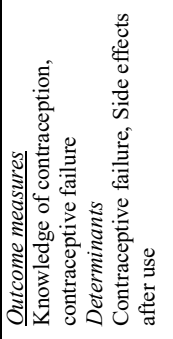 & 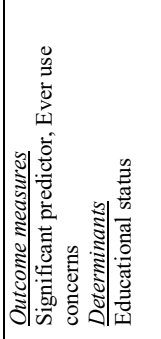 & 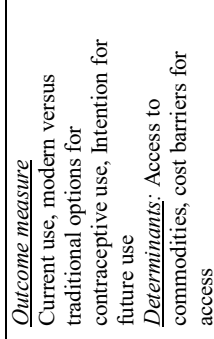 & 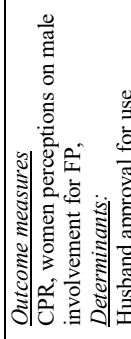 & 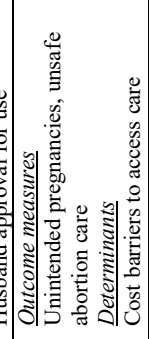 & 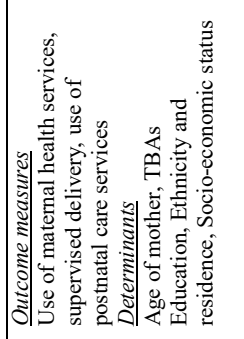 & 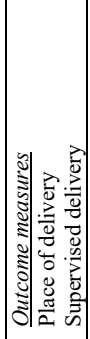 \\
\hline 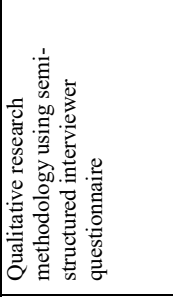 & 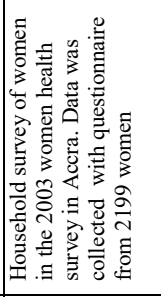 & 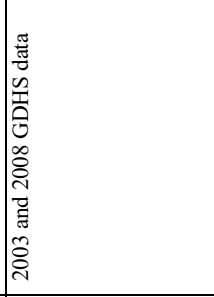 & 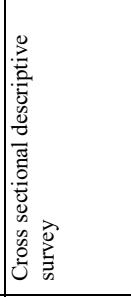 & 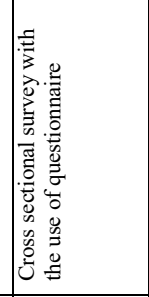 & 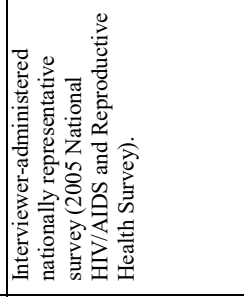 & 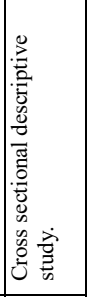 \\
\hline 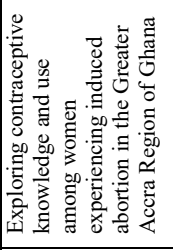 & 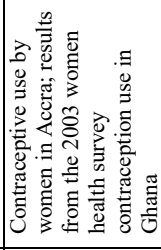 & 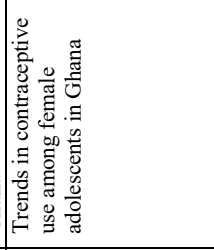 & 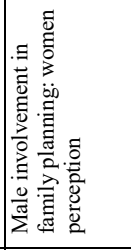 & 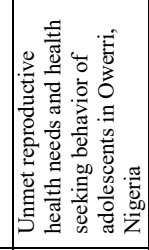 & 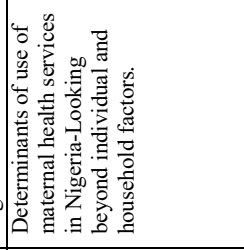 & 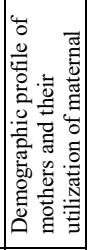 \\
\hline 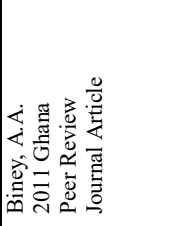 & 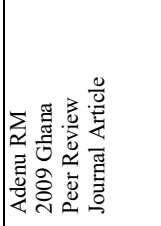 & 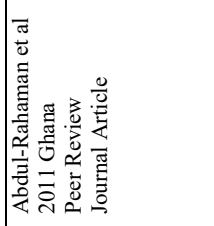 & 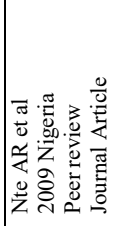 & 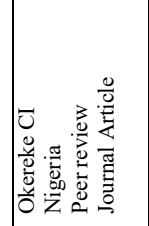 & 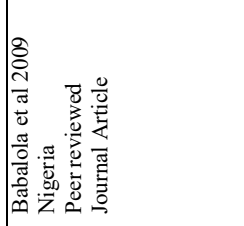 & 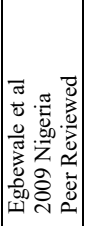 \\
\hline
\end{tabular}




\begin{tabular}{|c|c|c|c|c|c|c|}
\hline 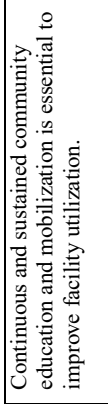 & 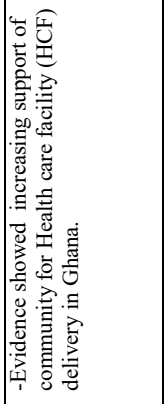 & 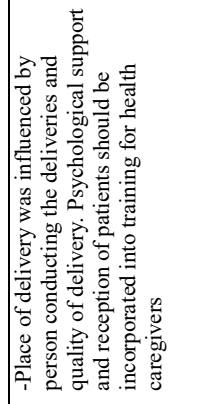 & 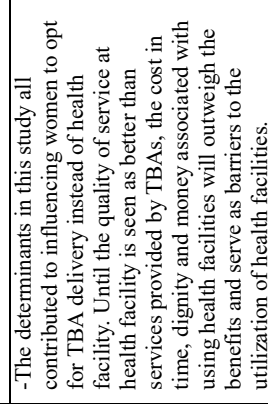 & 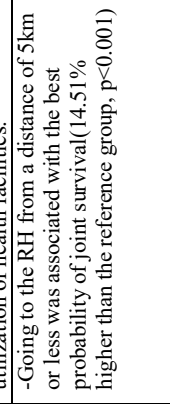 & 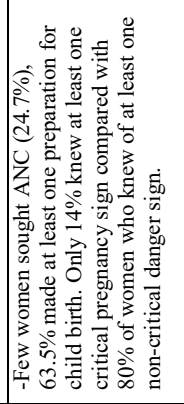 & 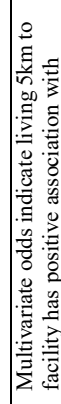 \\
\hline \begin{tabular}{l|l} 
\\
$\vdots$ \\
0
\end{tabular} & 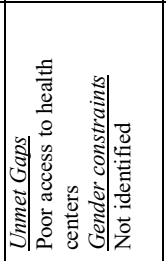 & 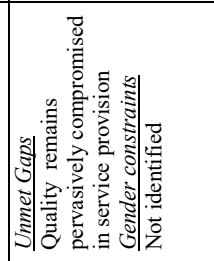 & 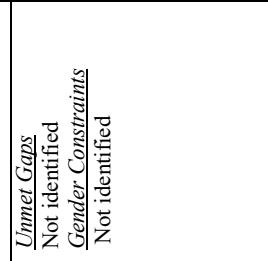 & 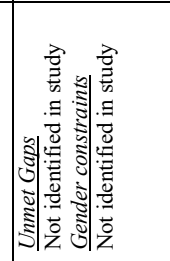 & 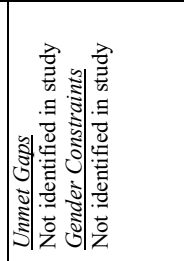 & \\
\hline \multirow[t]{2}{*}{ 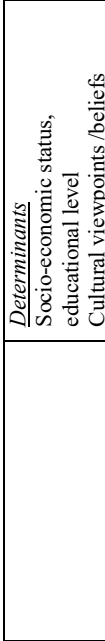 } & 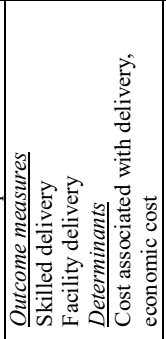 & 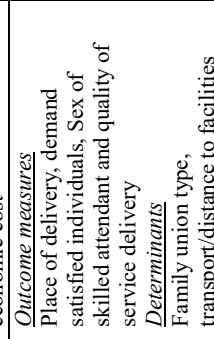 & 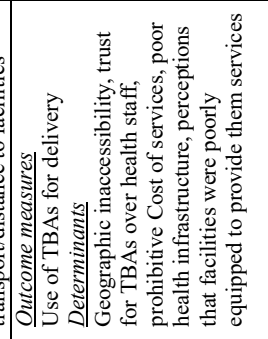 & 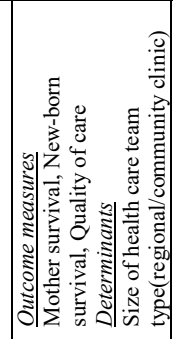 & 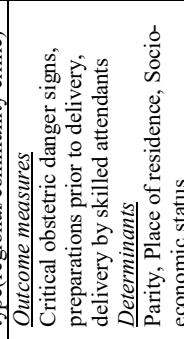 & \\
\hline & 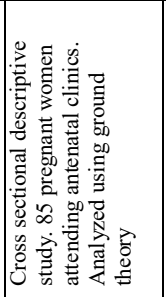 & 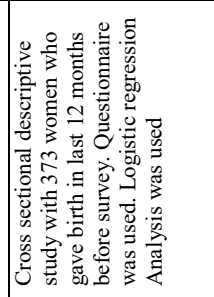 & 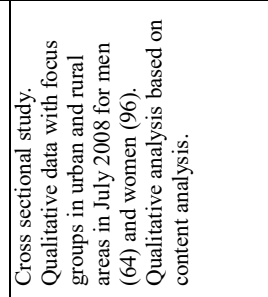 & 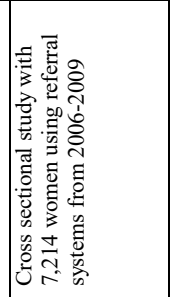 & 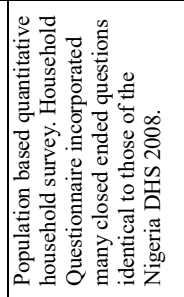 & 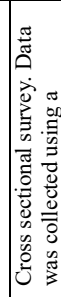 \\
\hline 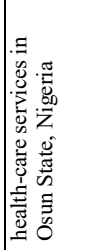 & 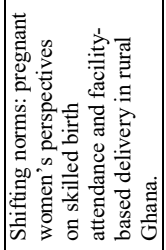 & 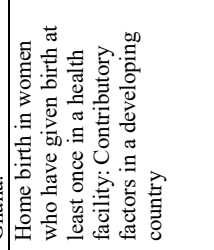 & 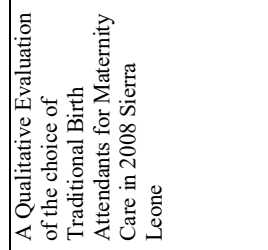 & 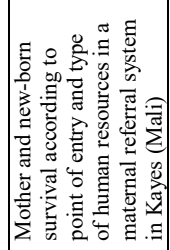 & 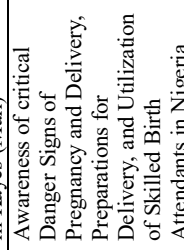 & \\
\hline 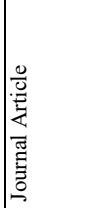 & 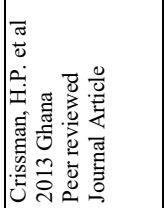 & 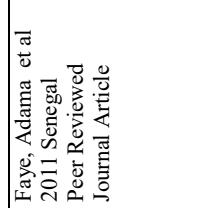 & 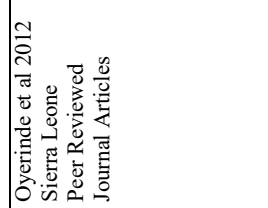 & 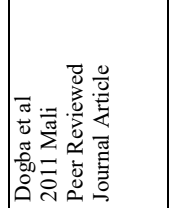 & 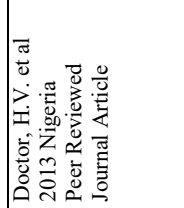 & \\
\hline
\end{tabular}




\begin{tabular}{|c|c|c|c|c|c|}
\hline 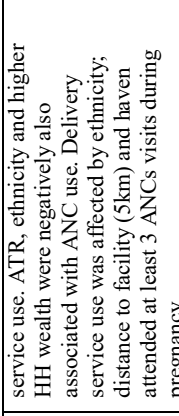 & 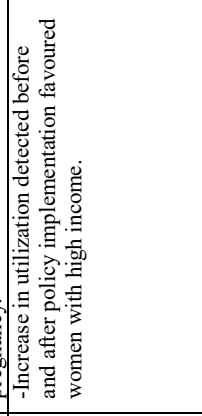 & 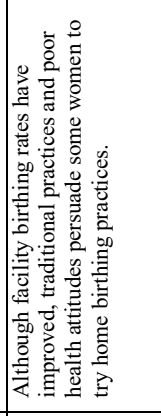 & 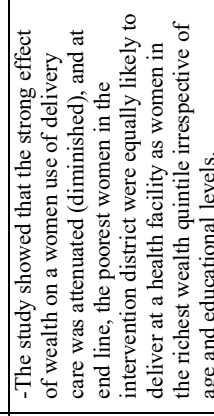 & 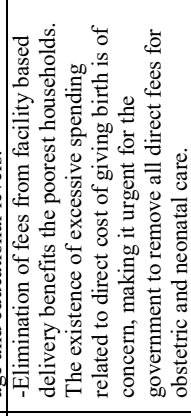 & 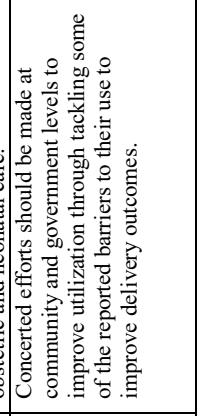 \\
\hline \begin{tabular}{l|l} 
\\
$:$
\end{tabular} & 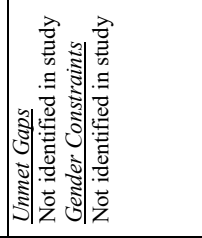 & 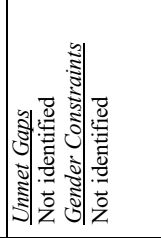 & (a) & 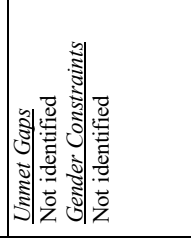 & 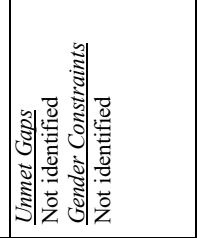 \\
\hline 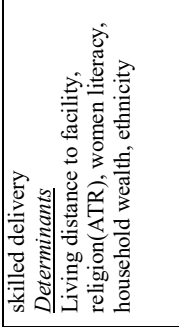 & 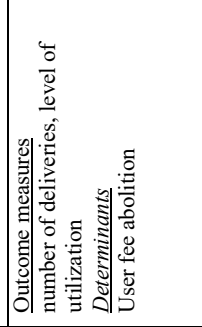 & 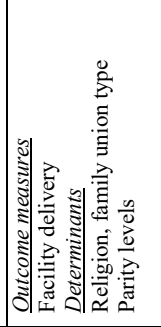 & 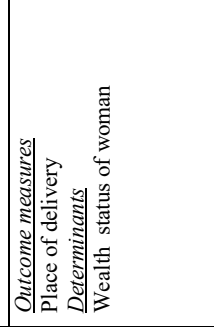 & 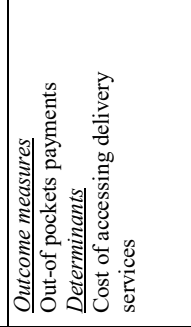 & 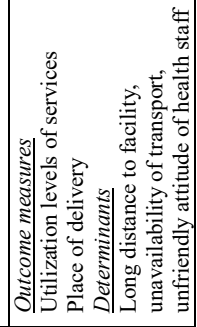 \\
\hline 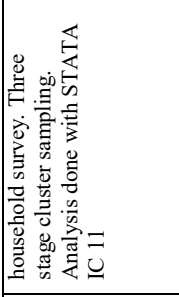 & 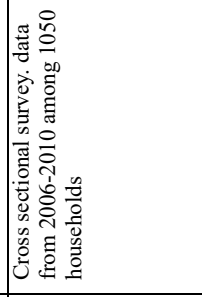 & 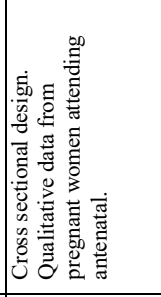 & 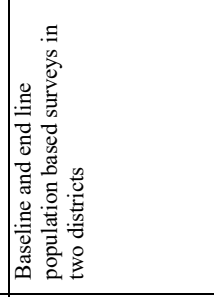 & 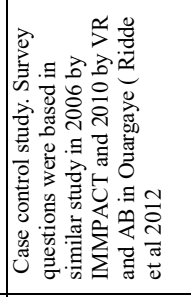 & 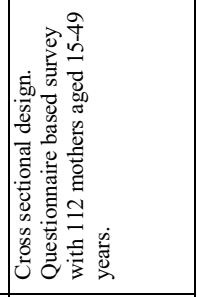 \\
\hline 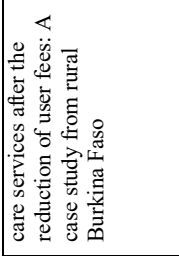 & 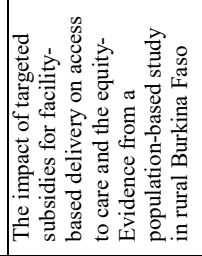 & 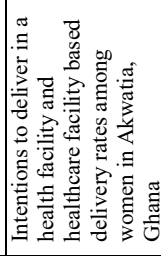 & 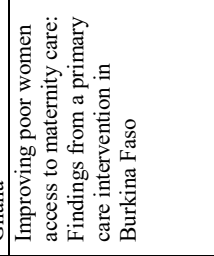 & 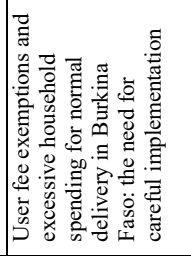 & 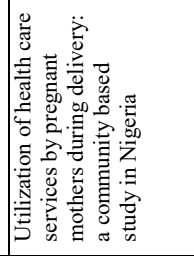 \\
\hline 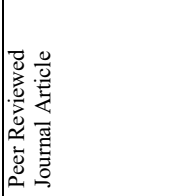 & 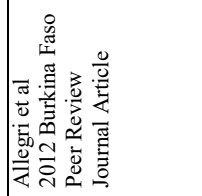 & 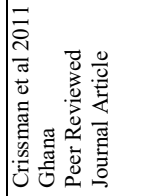 & 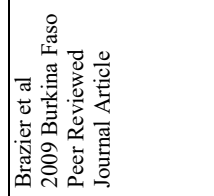 & 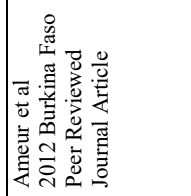 & 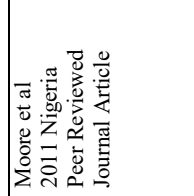 \\
\hline
\end{tabular}




\begin{tabular}{|c|c|c|c|c|c|c|}
\hline 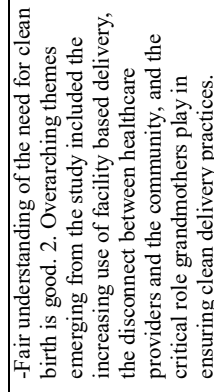 & 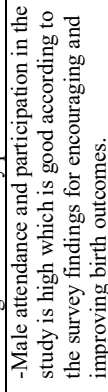 & 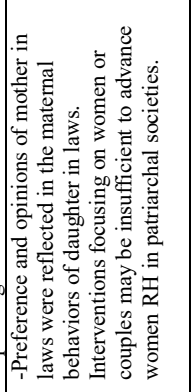 & 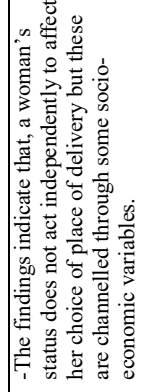 & 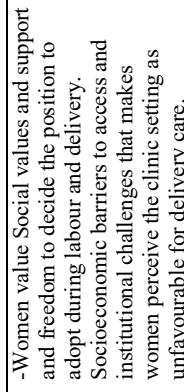 & 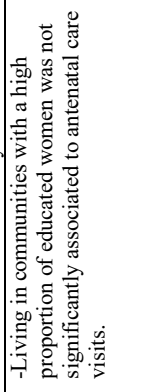 & 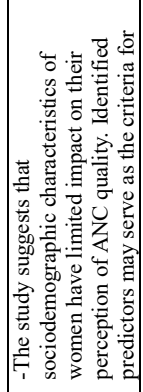 \\
\hline 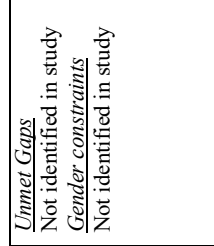 & 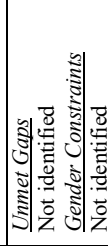 & 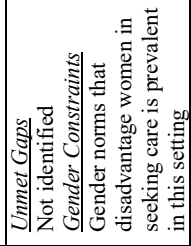 & 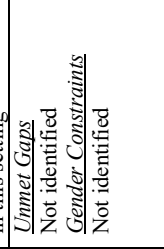 & 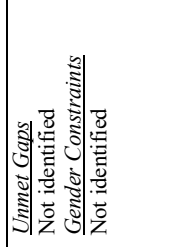 & 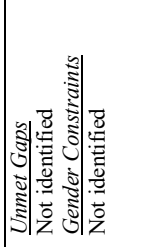 & 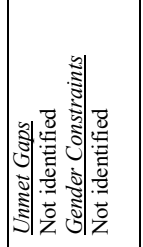 \\
\hline 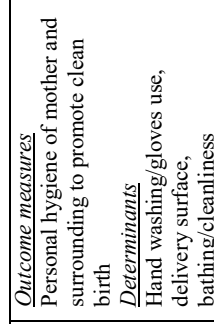 & 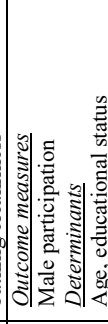 & 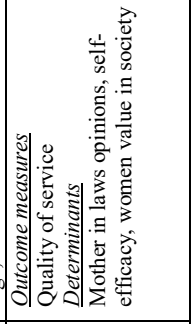 & 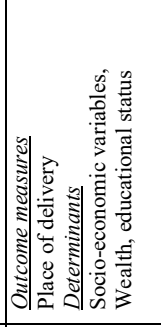 & 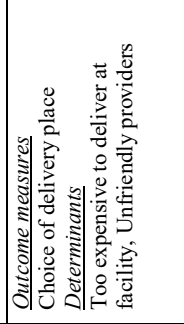 & 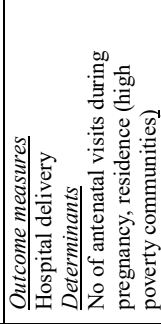 & 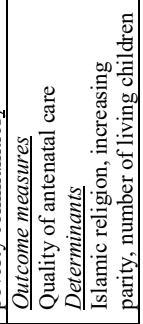 \\
\hline 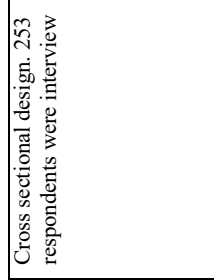 & 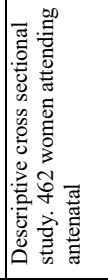 & 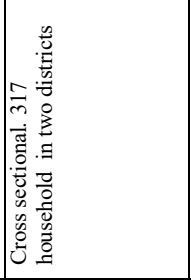 & 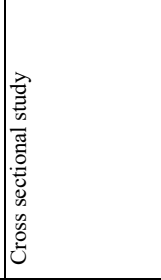 & 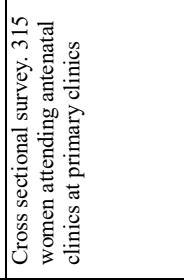 & 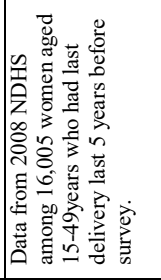 & 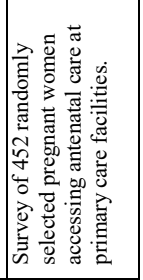 \\
\hline 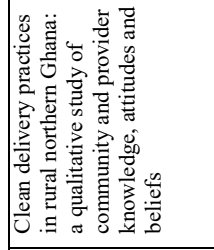 & 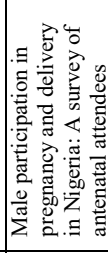 & 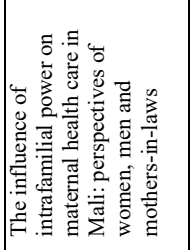 & 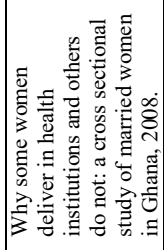 & 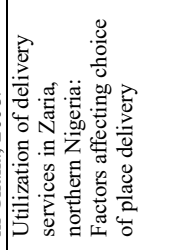 & 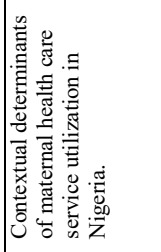 & 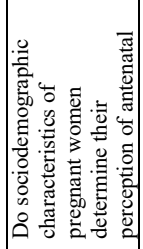 \\
\hline 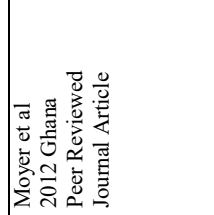 & 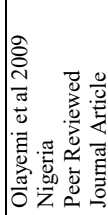 & 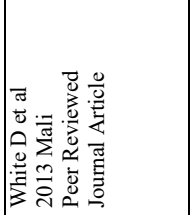 & 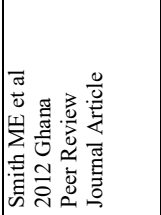 & 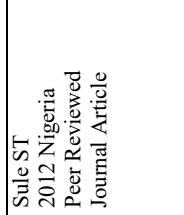 & 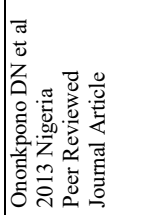 & 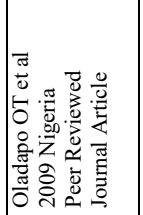 \\
\hline
\end{tabular}




\begin{tabular}{|c|c|c|c|c|c|}
\hline \multirow[t]{4}{*}{ 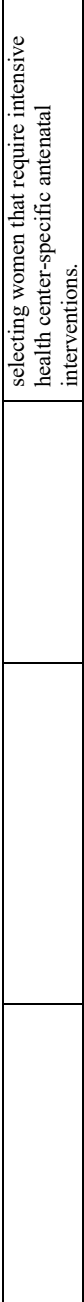 } & 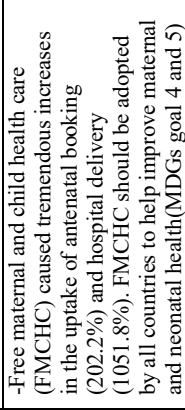 & 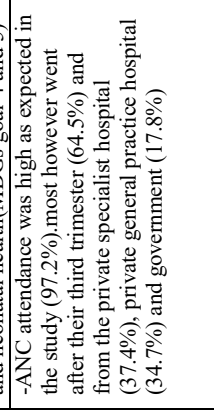 & 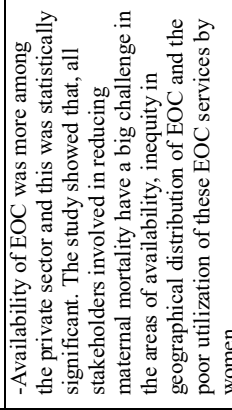 & 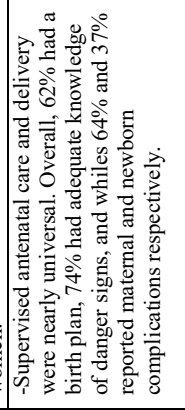 & 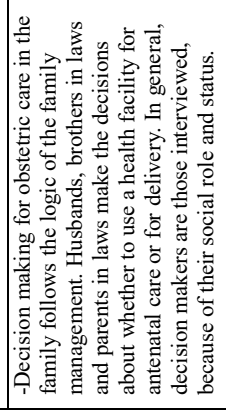 \\
\hline & 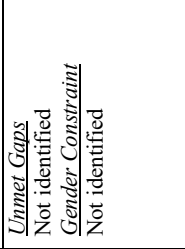 & 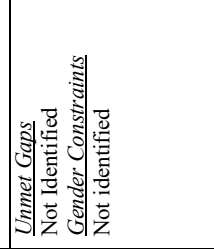 & 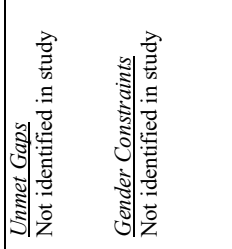 & 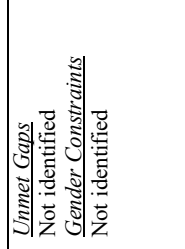 & 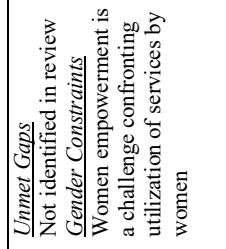 \\
\hline & 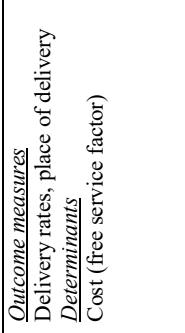 & 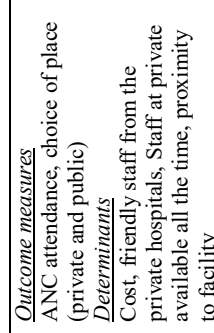 & 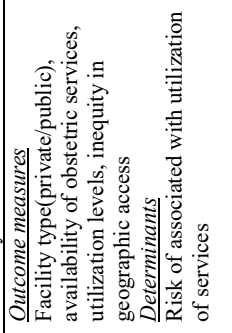 & 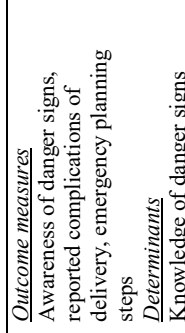 & 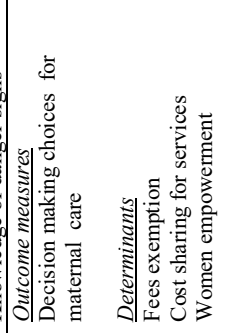 \\
\hline & 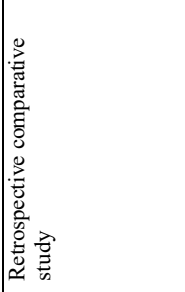 & 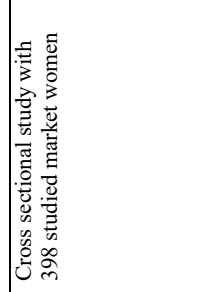 & 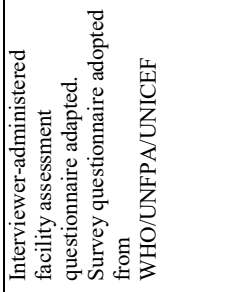 & 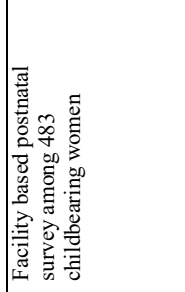 & 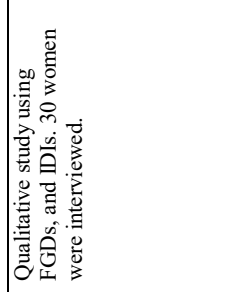 \\
\hline 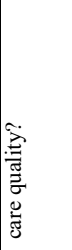 & 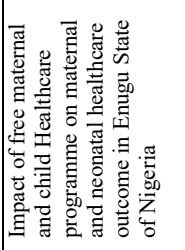 & 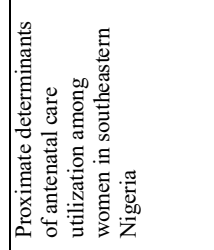 & 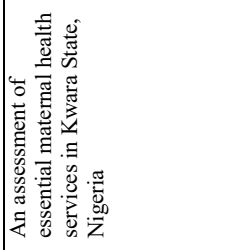 & 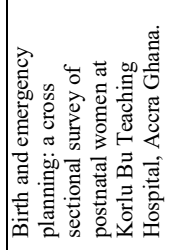 & 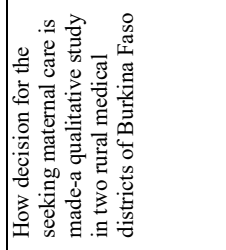 \\
\hline & 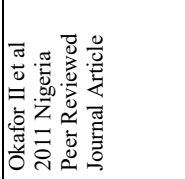 & 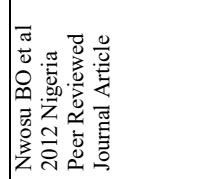 & 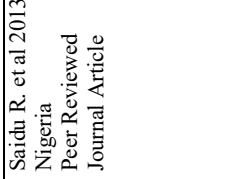 & 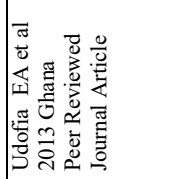 & 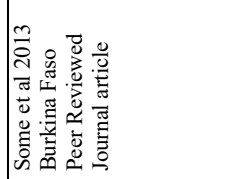 \\
\hline
\end{tabular}




\begin{tabular}{|c|c|c|c|c|c|}
\hline 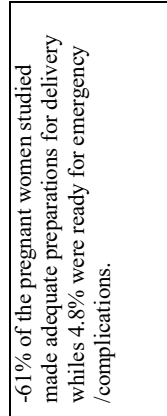 & 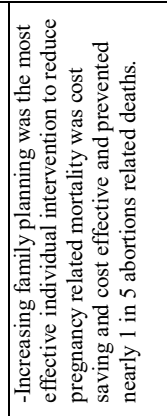 & 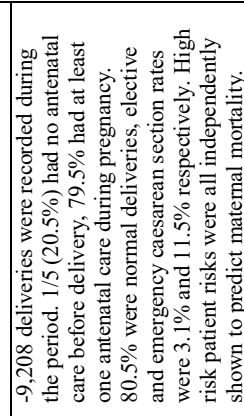 & 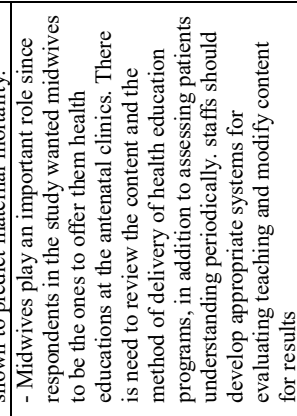 & 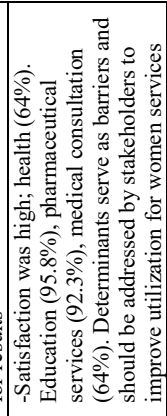 & 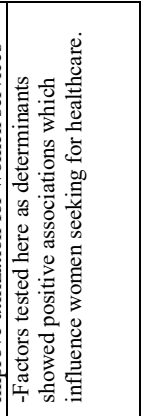 \\
\hline 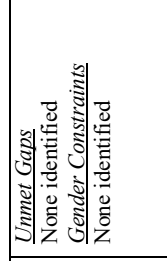 & 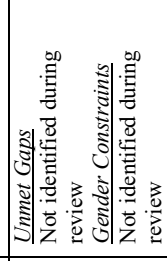 & 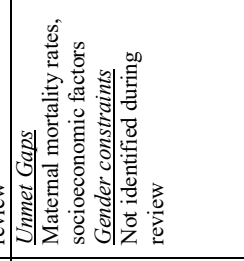 & 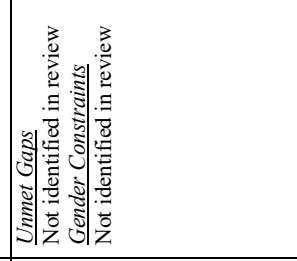 & 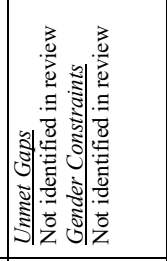 & 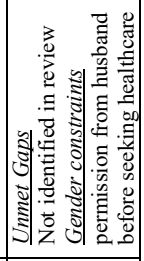 \\
\hline 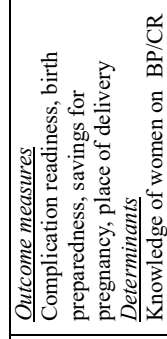 & 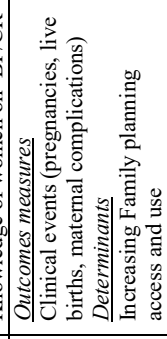 & 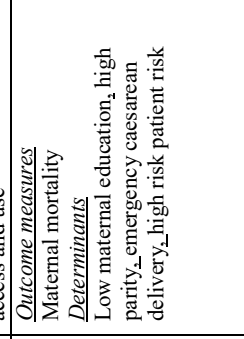 & 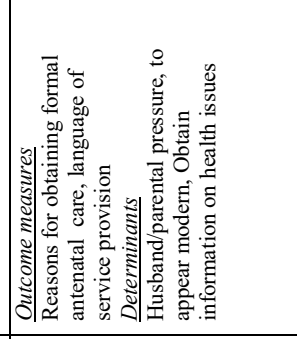 & 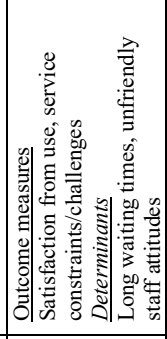 & 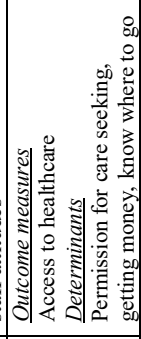 \\
\hline 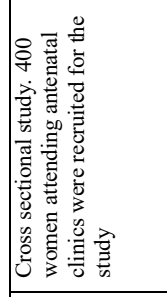 & 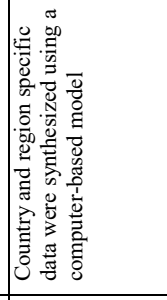 & 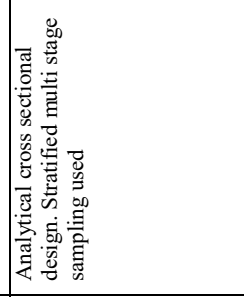 & 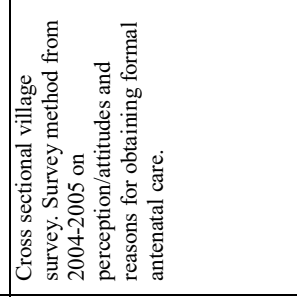 & 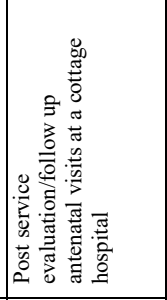 & 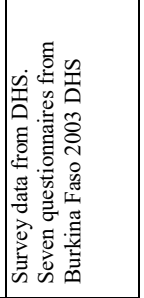 \\
\hline 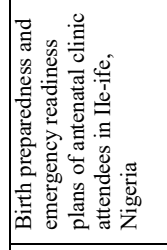 & 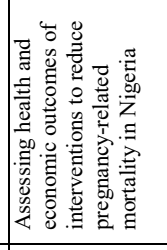 & 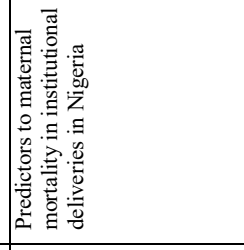 & 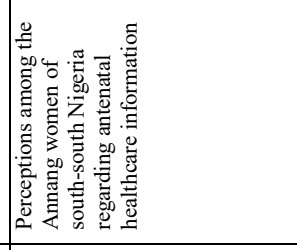 & 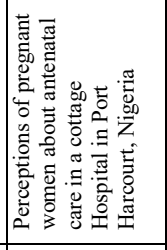 & 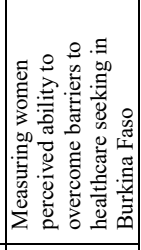 \\
\hline 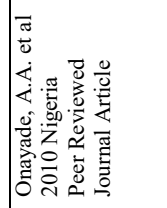 & 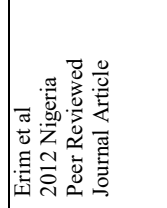 & 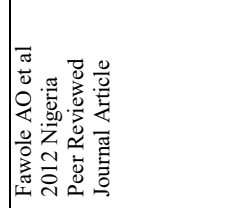 & 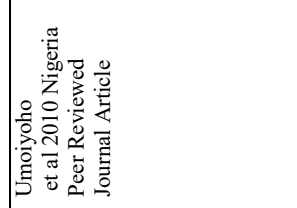 & 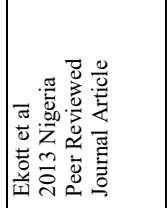 & 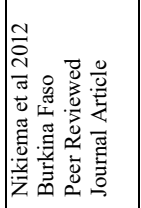 \\
\hline
\end{tabular}




\begin{tabular}{|c|c|c|c|c|}
\hline 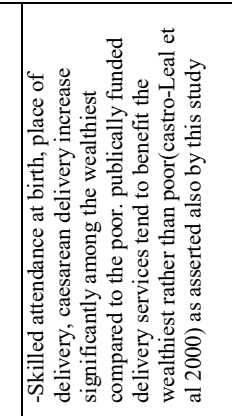 & 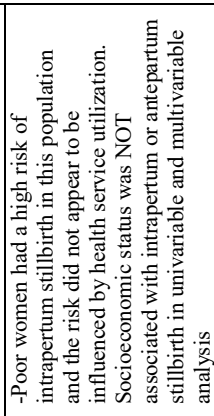 & 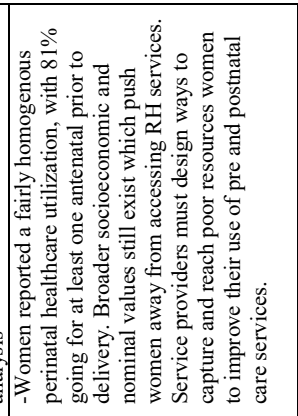 & 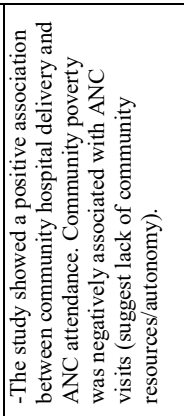 & 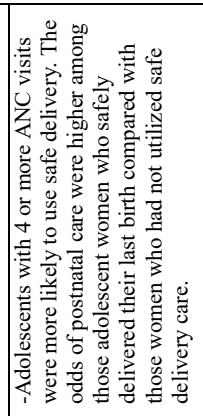 \\
\hline 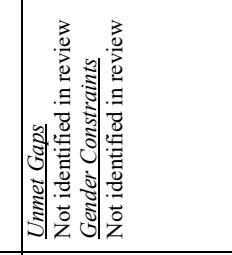 & 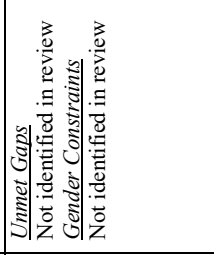 & 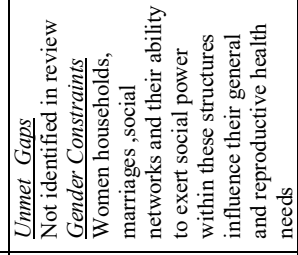 & 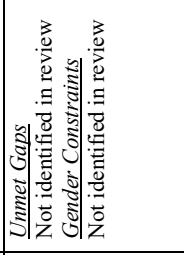 & 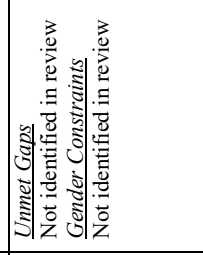 \\
\hline 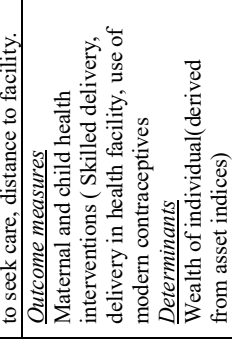 & 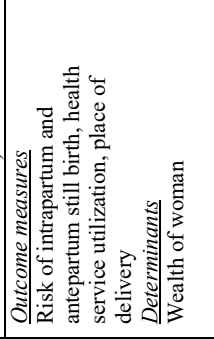 & 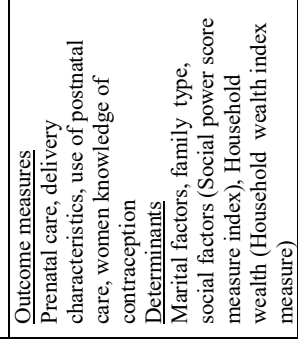 & 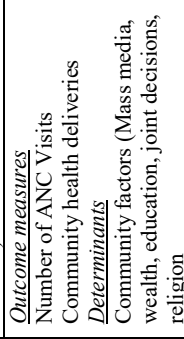 & 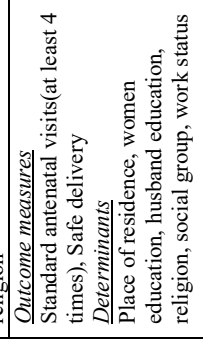 \\
\hline 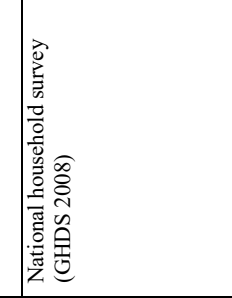 & 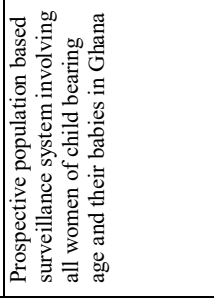 & 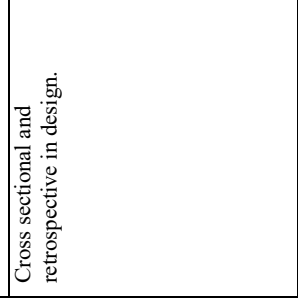 & 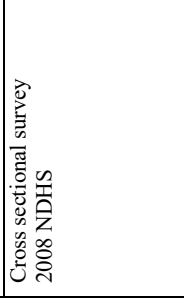 & 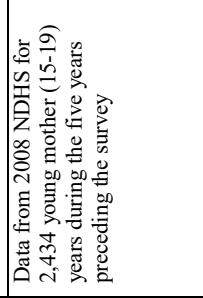 \\
\hline 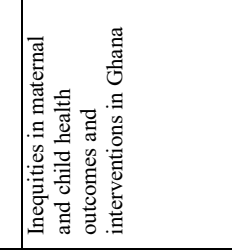 & 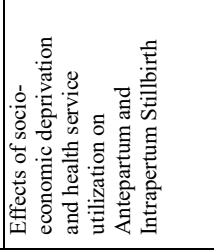 & 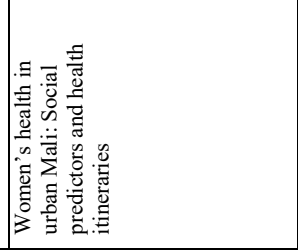 & 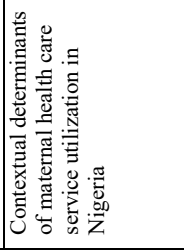 & 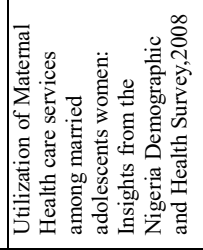 \\
\hline 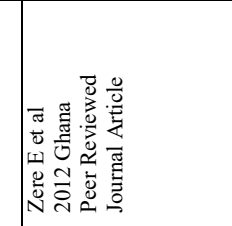 & 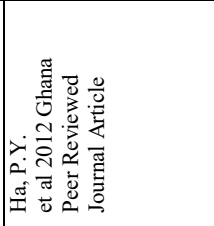 & 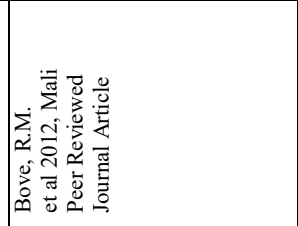 & 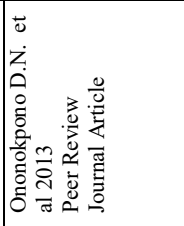 & 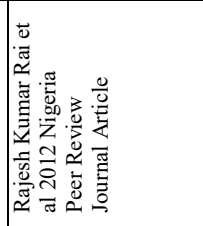 \\
\hline
\end{tabular}




\begin{tabular}{|c|c|c|c|c|c|}
\hline 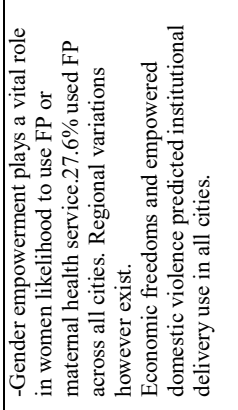 & 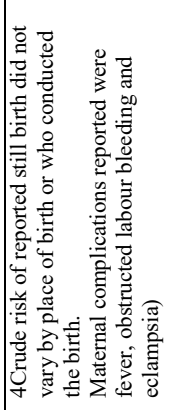 & 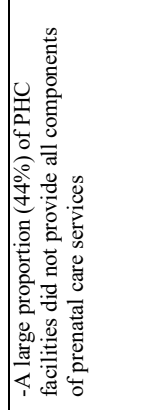 & 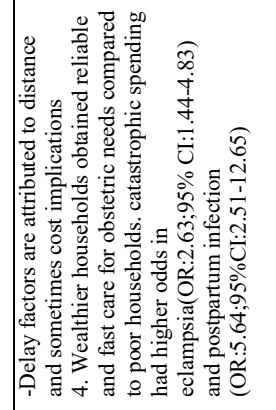 & 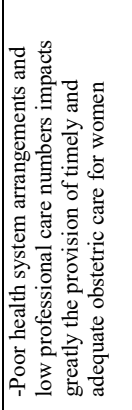 & 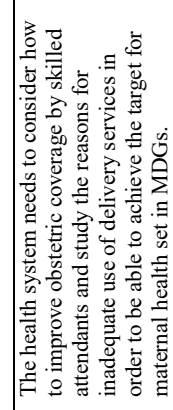 \\
\hline 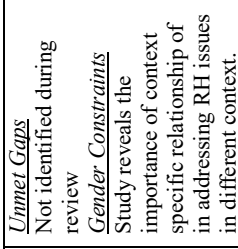 & 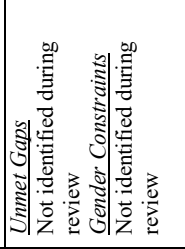 & 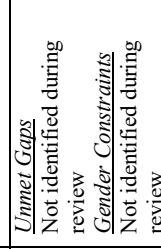 & 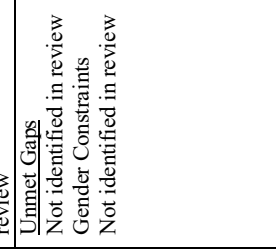 & 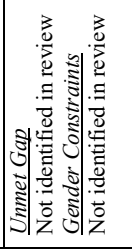 & 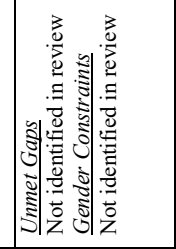 \\
\hline 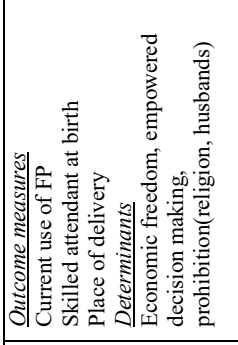 & 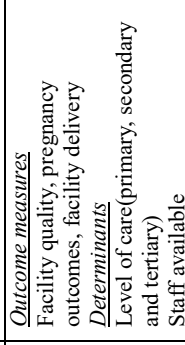 & 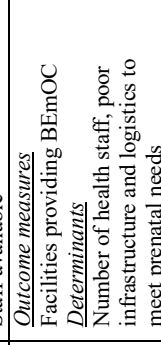 & 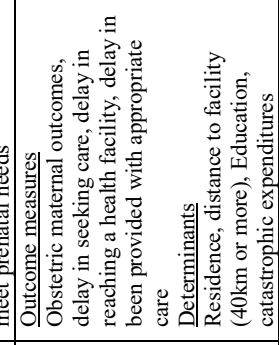 & 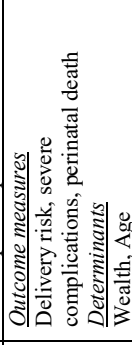 & 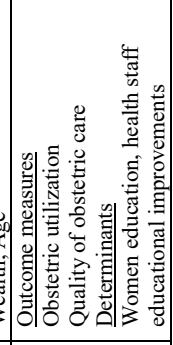 \\
\hline 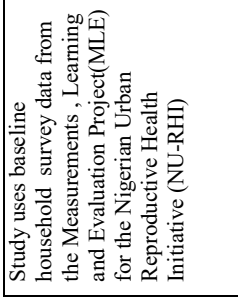 & 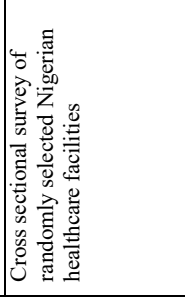 & 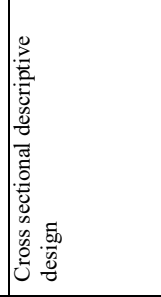 & 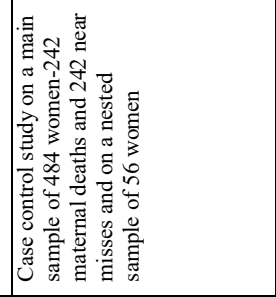 & 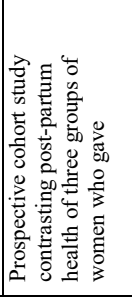 & 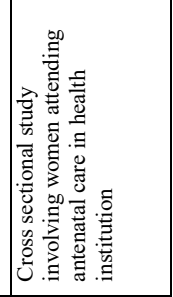 \\
\hline 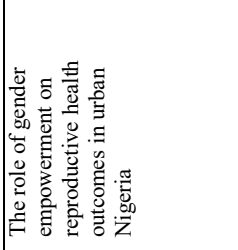 & 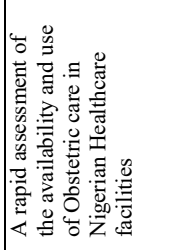 & 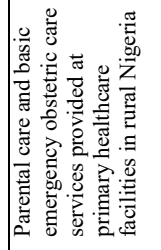 & 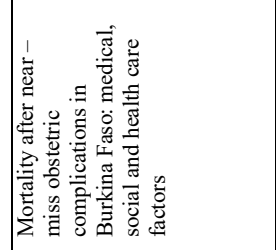 & 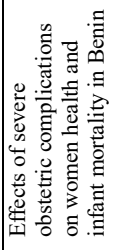 & 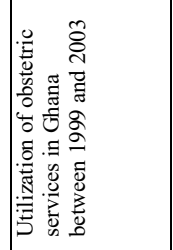 \\
\hline 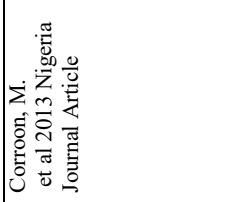 & 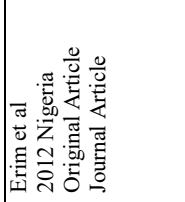 & 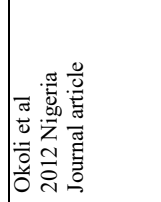 & 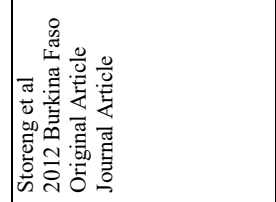 & 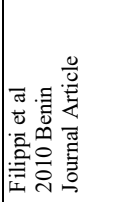 & 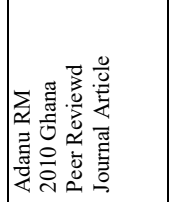 \\
\hline
\end{tabular}




\begin{tabular}{|c|c|c|c|c|c|c|}
\hline 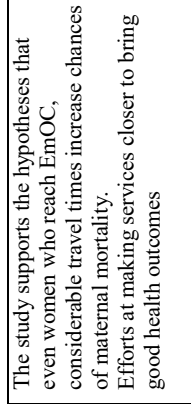 & 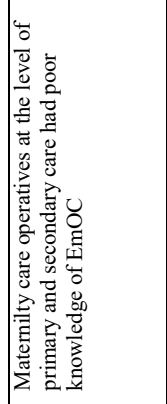 & 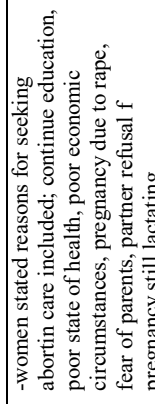 & 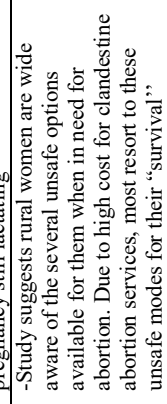 & 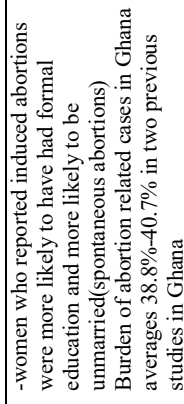 & 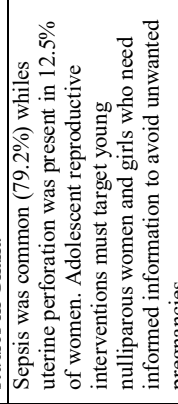 & 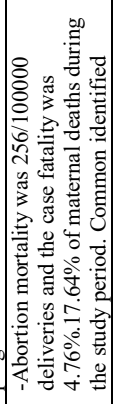 \\
\hline 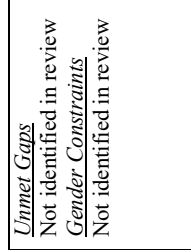 & 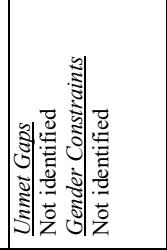 & 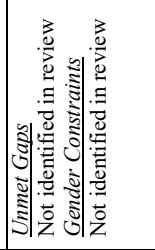 & 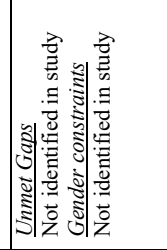 & 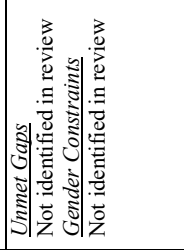 & 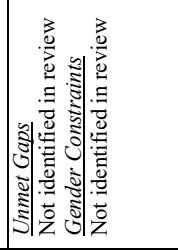 & 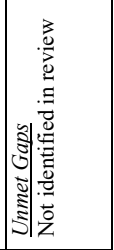 \\
\hline 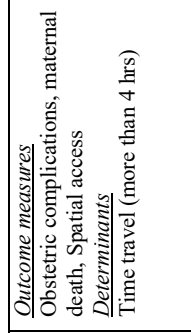 & 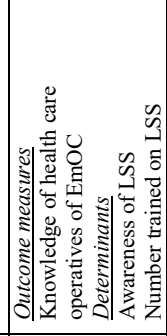 & 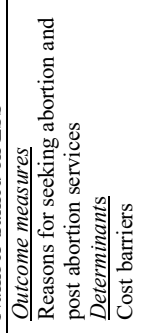 & 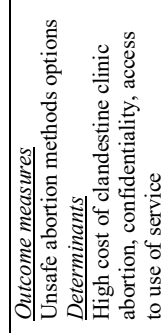 & 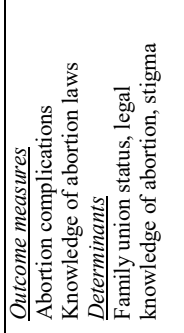 & 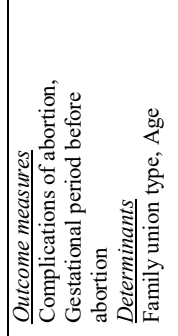 & 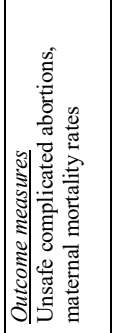 \\
\hline 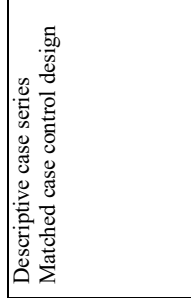 & 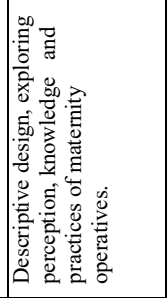 & 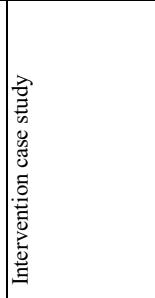 & 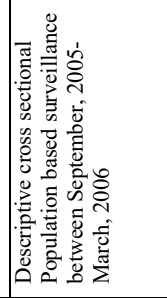 & 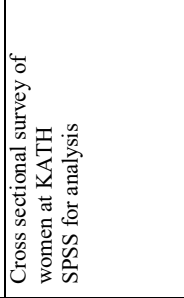 & 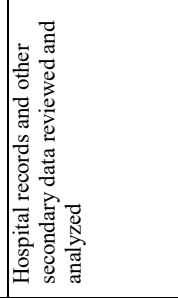 & 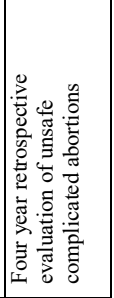 \\
\hline 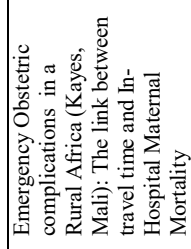 & 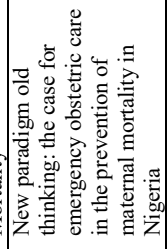 & 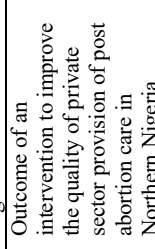 & 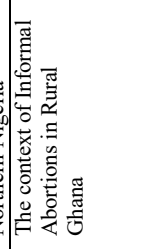 & 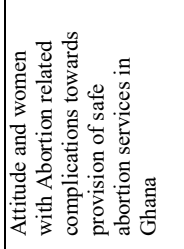 & 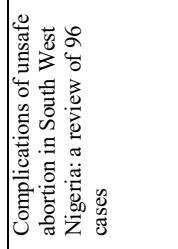 & 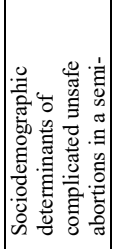 \\
\hline 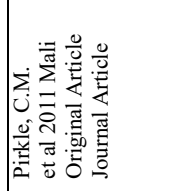 & 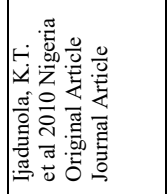 & 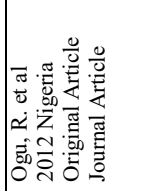 & 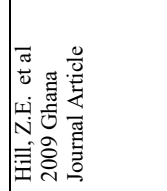 & 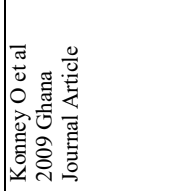 & 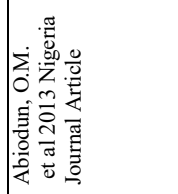 & 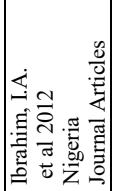 \\
\hline
\end{tabular}




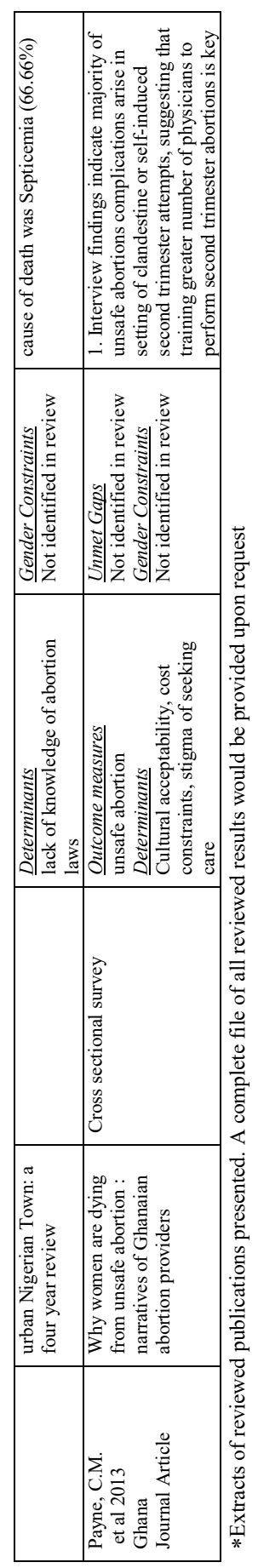


Socio-demographics of respondents/cluster analysis results applied in Chapter 3 


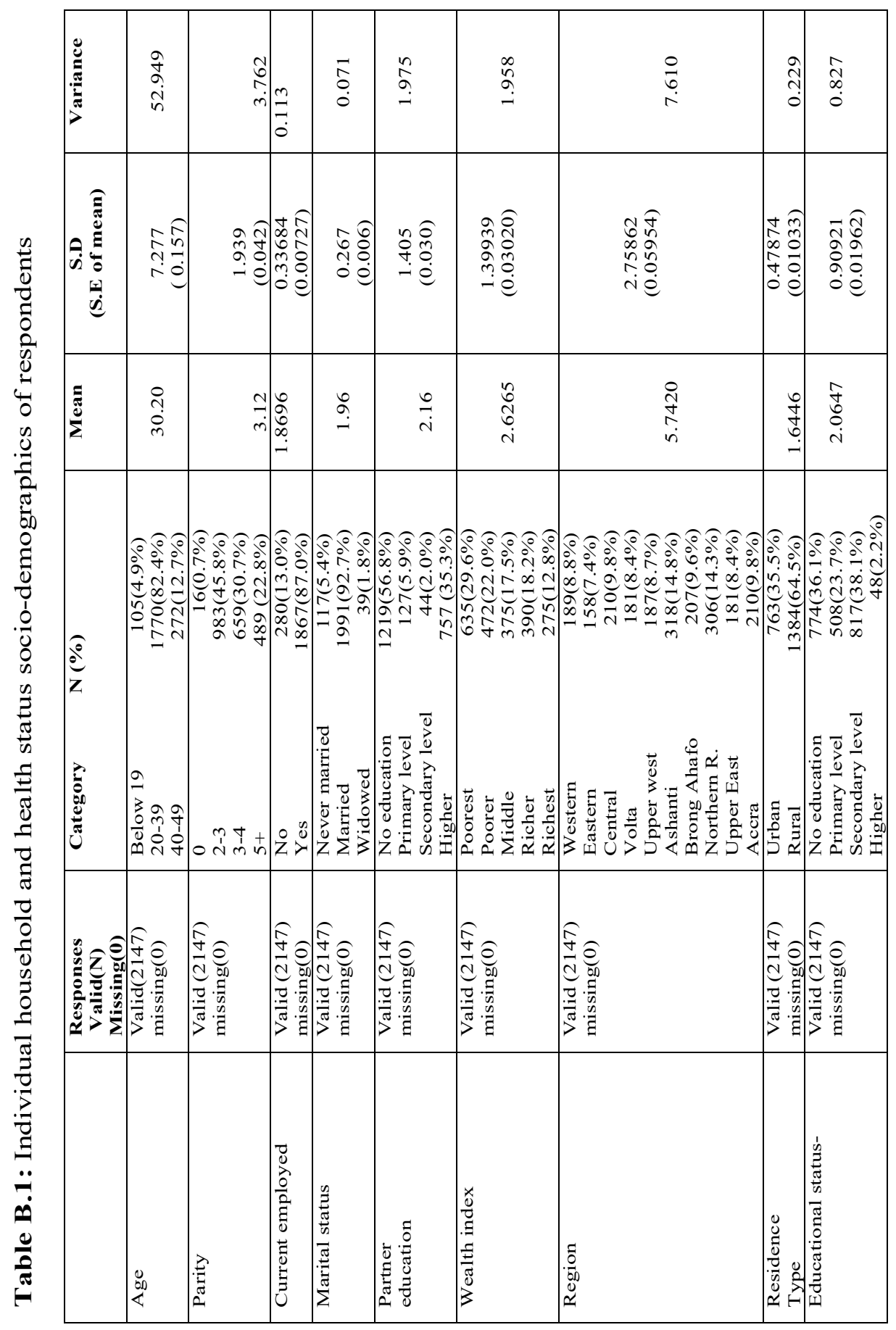




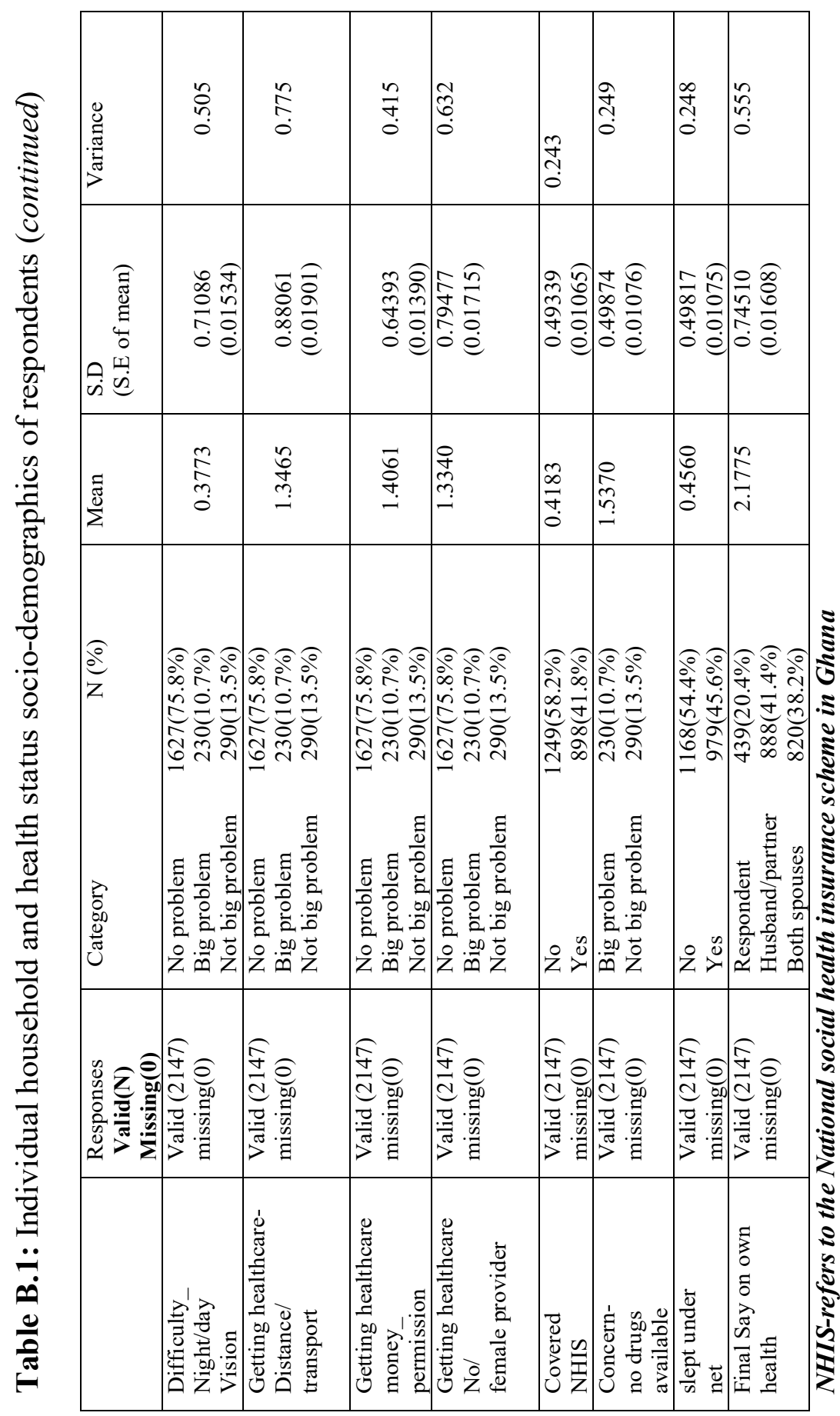




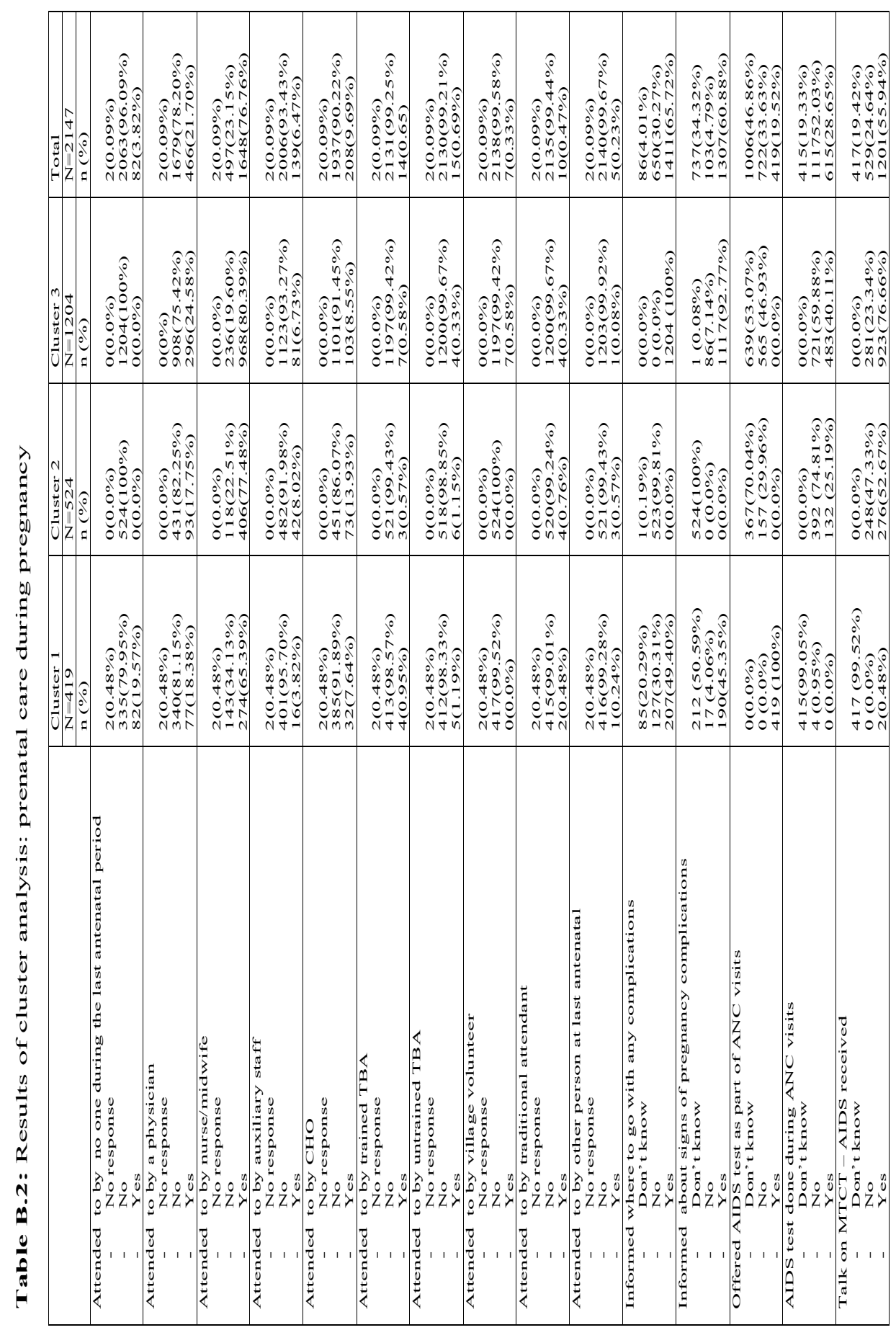




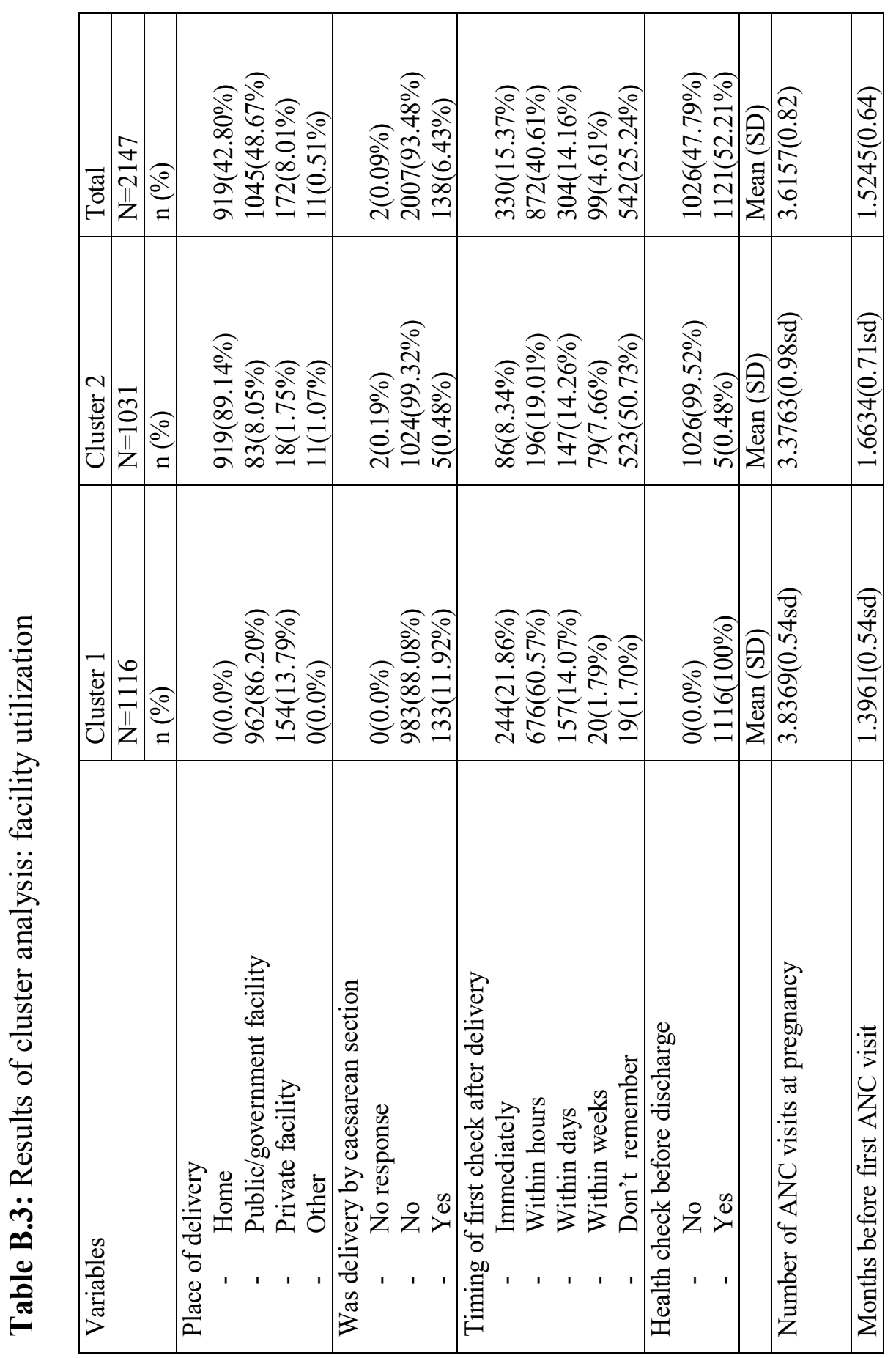




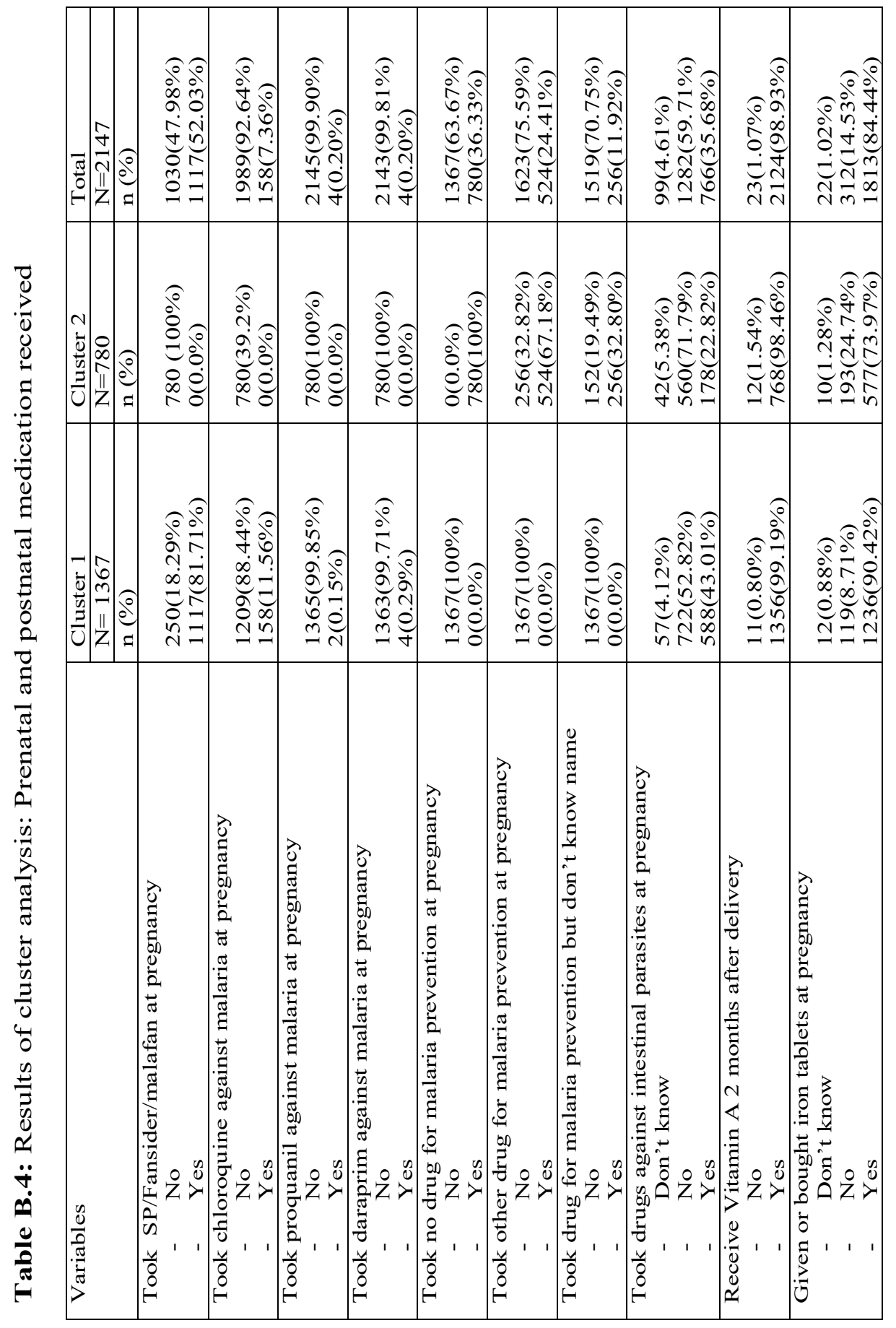




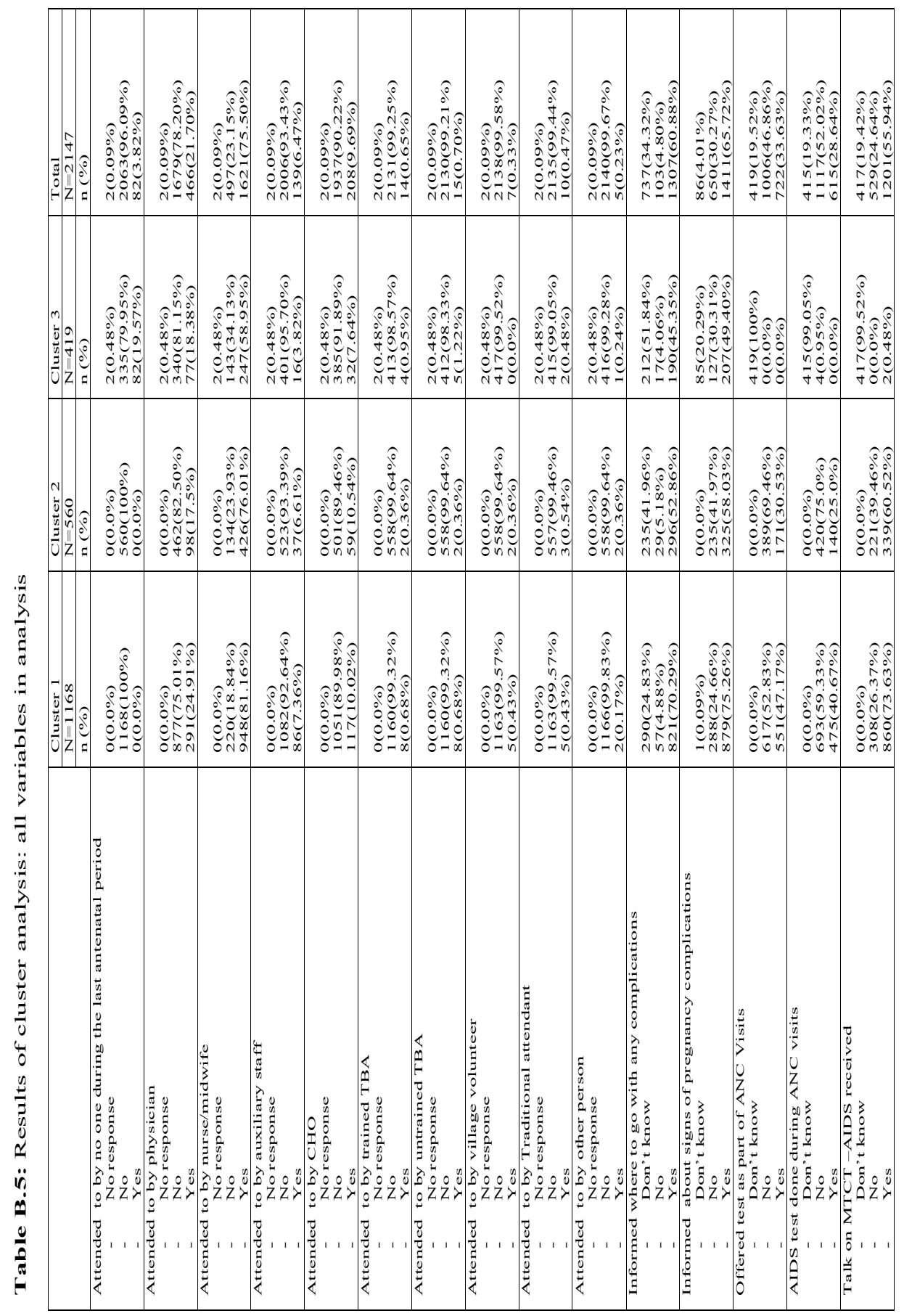




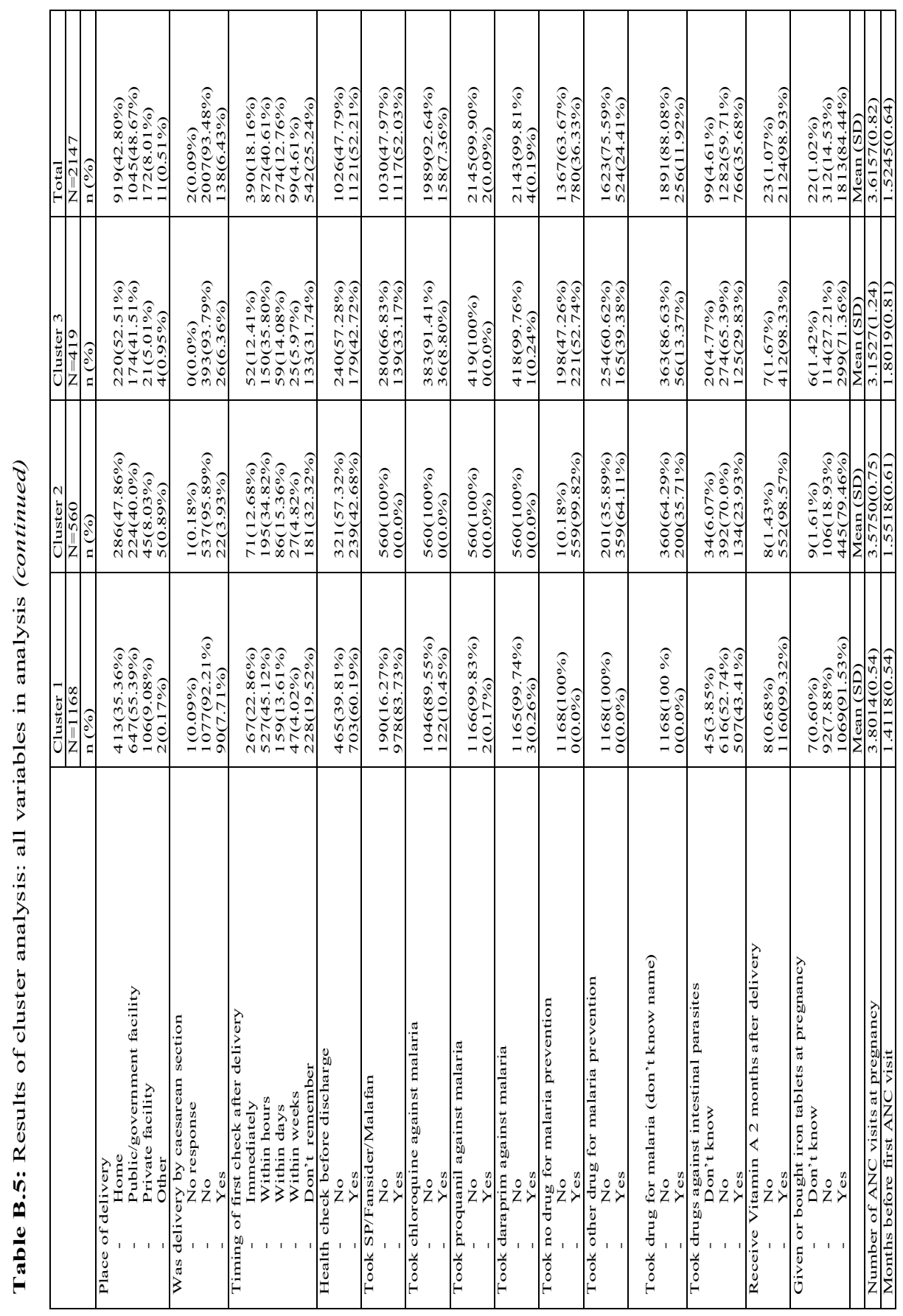



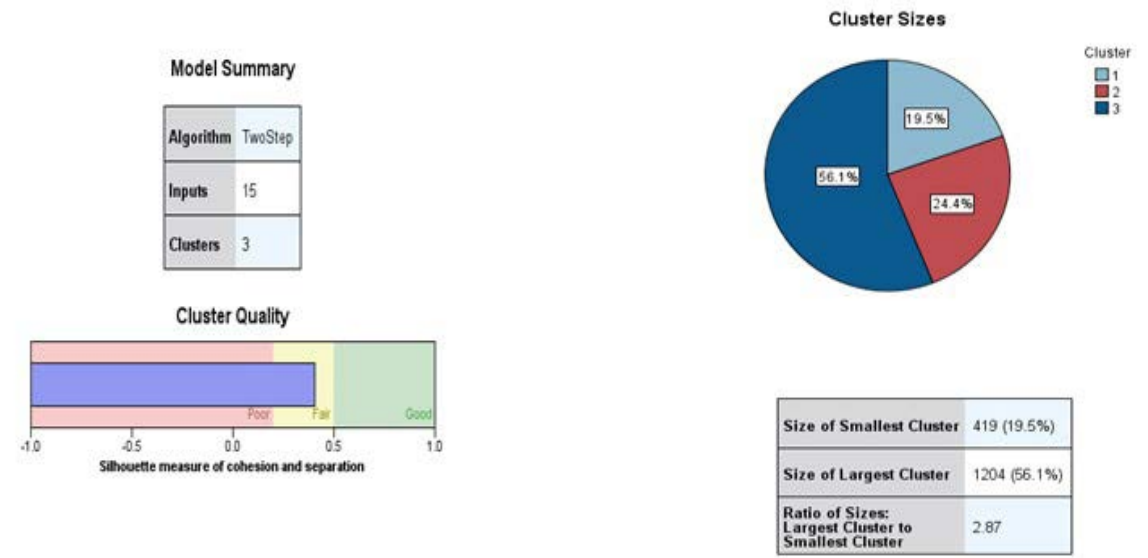

Prenatal and postnatal care variables
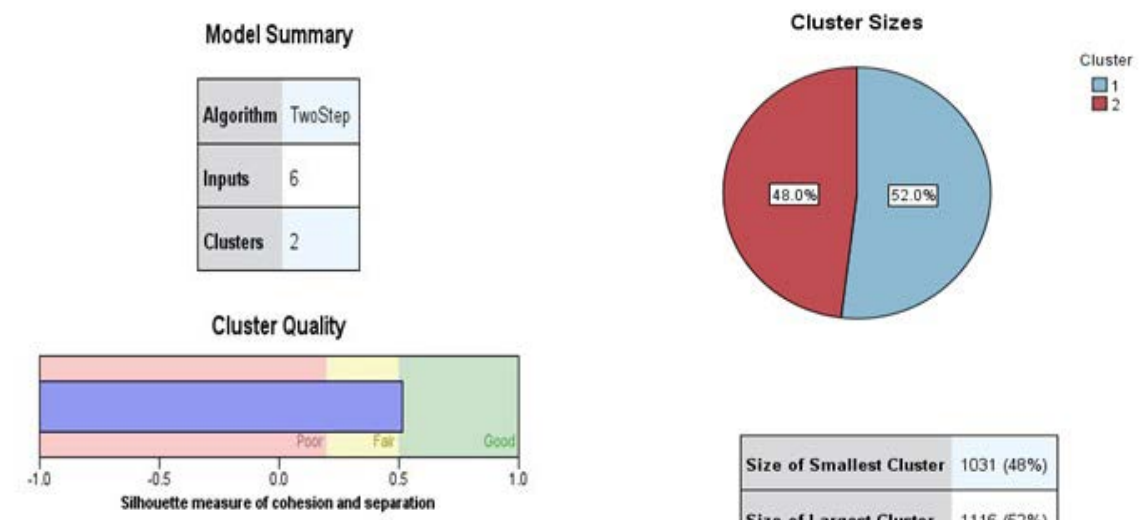

\begin{tabular}{|ll|}
\hline Size of Smallest Cluster & $1031(48 \%)$ \\
\hline Size of Largest Cluster & $1116(52 \%)$ \\
\hline $\begin{array}{l}\text { Ratio of Sizes: } \\
\text { Largest Cluster to } \\
\text { Smallest Cluster }\end{array}$ & 1.08 \\
\hline
\end{tabular}

Facility Utilization and care variables 


\section{Model Summary}

\begin{tabular}{|l|l|}
\hline Algorithm & TwoStep \\
\hline Inputs & 10 \\
\hline Clusters & 2 \\
\hline
\end{tabular}

Cluster Quality

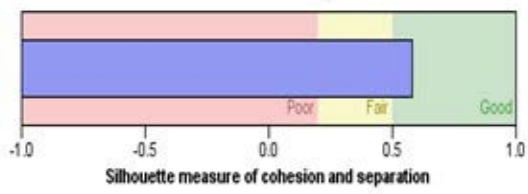

Cluster Sizes

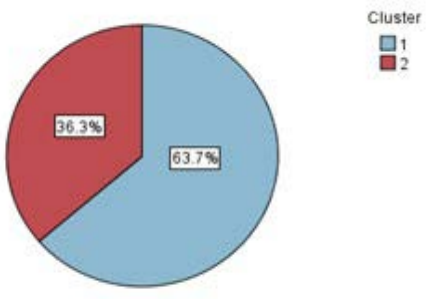

밈

\begin{tabular}{|l|l|}
\hline Size of Smallest Cluster & $780(36.3 \%)$ \\
\hline Size of Largest Cluster & 1367 (53.7\%) \\
\hline $\begin{array}{l}\text { Ratio of Sizes: } \\
\text { Largest Cluster to } \\
\text { Smallest Cluster }\end{array}$ & 1.75 \\
\hline
\end{tabular}

Prenatal and postnatal care medication variables

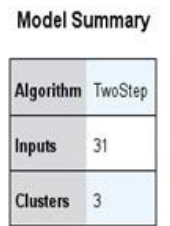

Cluster Quality

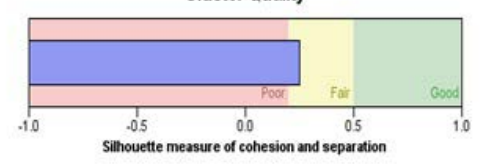

All response variables in cluster analysis

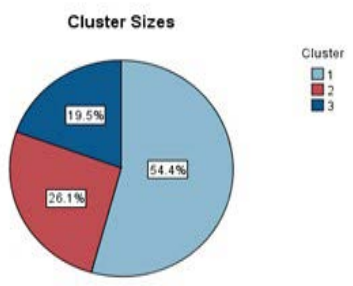

\begin{tabular}{|l|l|}
\hline Size of Smallest Cluster & $419(19.5 \%)$ \\
\hline Size of Largest Cluster & $1168(54.4 \%)$ \\
\hline $\begin{array}{l}\text { Ratio of Sizes: } \\
\text { Largest Cluster to } \\
\text { Smallest Cluster }\end{array}$ & 2.79 \\
\hline
\end{tabular}




\section{Appendix C \\ Wording of sample questions applied in Chapter 4}


Chapter 4 (Shortcomings for maternity care in rural Ghana)

\section{C.1 Sample qualitative (Women component)}

\section{Service provision related shortcomings}

1. What kind of services do you seek or receive from facility care providers? Probe further to find out who are the actual birth attendants, both with and outside the organized health care system

2. What components of delivery care are provided at your facility level? Which are excluded and why? Are there challenges in providing these critical services for women at point of use?

3. Do you have access to obstetric/abortion care services at your facility? What levels of these services are provided and WHY? If currently not receiving? What is the level of

4. "Focused ANC" is implemented now at your facility level; how does this improve/affect service provision you receive now? Probe for noTable differences between former and later services

\section{Lifesaving skills and knowledge shortcomings}

5. Averagely, how many of these people provide these services at the facility/clinic/community level? Probe further for numbers on nurse-density-to-population ratio and type of personnel(skilled and unskilled) providers

6. If unskilled personnel (trained or untrained TBAs etc. mentioned), probe why they exist and why women prefer their services?

\section{Quality, cost and satisfaction shortcomings}

7. Is the type and quality of care you receive associated with social and financial status within the community? Probe further for clarity of views provided for or against any responses

8. What can you say on the cost (affordable or not affordable) associated with expectant women who deliver at the facility level? Does it encourage or discourage good quality delivery care the point or delivery or NOT?

Are there any views on financial constraints you incur that somebody has something to say?

9. What are common maternal complications of delivery that you faced the last time and were they resolved to your satisfaction? Are obstetric care services readily available as and when you need them? Probe further on abortion and post abortion care services availability and use

\section{System level shortcomings and consensus on maternity care}

10. At your last pregnancy, how long did it take you to visit the facility or health provider for the first time and why? Probe further on first visitation and reasons accounting for early or delay

11. What heath care system issues (successes and challenges) affects maternity care services for you? Probe further for clarity of responses on this.

12. Can you provide further information on those who are actual birth attendants both within and outside the health care facility level that cater for your maternity health needs?

13. Is it easier to seek ANC services then delivery services at the facility level? Provide evidence from GDHS reports and probe further on women views.

14. Probe on women views on "third delay for seeking maternity care concept"; providing prompt services at facility level. Further exploit this concept for women views 
C.3 Sample qualitative (Policymakers component)

\section{Service provision shortcomings}

1. What "push" or "pull" factors account for service delivery and utilization at the facility level and WHY?

2. Service provision for women has challenges such as.... Probe on policy gaps and implementation strategies

Among others; what steps are implemented to improve this?

3. What components of birthing care have major challenges at the facility level? What steps are been implemented to address these in the short, medium and long term?

4. "Focused ANC" is implemented now by MOH/GHS; how does this improve/affect service provision at facility level?

5. What in your views account for rising ANC attendance and low skilled delivery at birth? What is the MOH/GHS policy in addressing these gaps? Provide further evidence from recent GDHS reports for provider's views. What

\section{Lifesaving skills and knowledge shortcomings}

6. You mentioned trainings for health staff to deliver appropriate services. Do you consider them adequate for health providers? Do you also provide beneficiary users trainings on effective utilization and at what level (individual/community)? Do you consider these trainings highlighted adequate? How often are they provided?

7. Do you consider current policies on reproductive health (sexual rights and gender based violence) and maternity care (skilled delivery and obstetric care) adequate within the MOH/GHS at both supply and demand side? Prompts for implementation progress, successes, shortfalls, possible revisions

8. In delivering "Focused ANC" care at the facility level, what lifesaving skills and knowledge has the MOH/GHS provided to health providers to ensure effective implementation of these services for women?

\section{Ouality, cost and satisfaction shortcomings}

9. As (......... here mention position), what do you consider important milestones attained to meeting the delivery of efficient, quality and effective reproductive and maternity care services? Prompts for quality, efficiency and client centred attributes on skilled delivery and obstetric care services

10. What mechanisms exist within the MOH/GHS in ensuring the delivery of quality, satisfactory and cost effective services for maternity care services?

11. Are you aware of women accounts of out- of- pocket payments/unofficial payments at facility level before been provided the services they need? What measures is MOH/GHS currently undertaking to ensure cost does not unduly affect women access and use for skilled birthing and obstetric care?

\section{System level shortcomings and consensus}

12. What monitoring mechanisms exist for staff effective delivery of policies and protocols at the user and provider levels?

13. What do you consider major barriers and facilitators for service delivery of the components of maternity and reproductive rights? Prompts: resources, infrastructure, HR issues-staffing, recruitment, staff trainings, motivation/attitudes for health staff

14. What factors impact staff motivation and performance on delivery maternity and reproductive health services in the district or region? Are knowledge levels of staff adequate in the delivery of these services? Prompts for staffing knowledge, skills, and trainings provided

15. Are there major consensuses you have to indicate between policy makers, health providers, women users and other non-state actors towards delivery maternity services spoken about?

16. Is there anything else you would like to say concerning maternity care in the (....district/region) not spoken off? 



\section{Appendix D}

Sample questions applied in Chapter 5 
D.1: (Quantitative data use)

\section{Section A}

\section{Social and demographic characteristics}

We will like to find out about issues concerning your social and demographic characteristics. This data will enable us statistically assess the proceeding issues on contraceptive use.

Q.1 What is your current age as a mother?

Q.2 At what age did you have your first child?

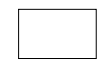

Q.3 What is your current marital status?

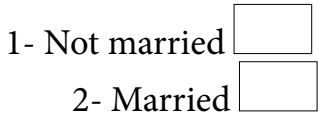

Q.4 What family Union do you belong to?

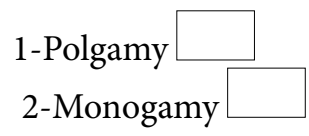

Q.5 What is your highest level of Education?

$1-$ Never educated $\square$

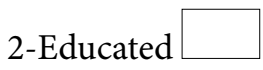

Q.6 Does your religion have an effect on your contraceptive use?<smiles>[Y19]C1CCC1</smiles><smiles>C1CCCCC1</smiles>

Q.7 Does your social norms have effects on your contraceptive methods use?

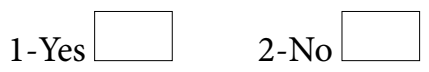

Q.8 What is your current employment status?

1-Unemployed $\square$ 2-Employed $\square$

Q.9 How many children are you currently living with? 


\section{Section A}

\section{Current and ever use of contraception}

This section assess current and previous use issues, drawing on your decision making choices, information seeking and cost related factors for use of contraception currently or previously.

Q.1Have you and any partner used any contraceptive method before<smiles>[Y19]1CCC1</smiles><smiles>[Y6]N1CCC1</smiles>

Q.2 Are you currently using any contraceptives<smiles>[Y19]C1CCC1[AsH2]</smiles>

Q.3 Where did you first hear a message about the use of contraception
1-Radio, $\square$

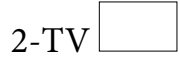
3-Heath facility $\square$
4-Other

Q.4 What method of family planning do you know about

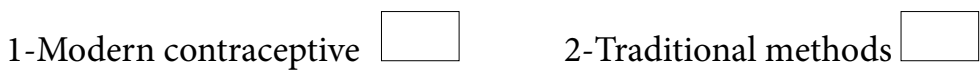

Q.5 Which sources do you hear/know about family planning

1-clinic $\square$

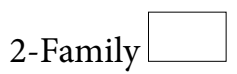

3-Friends $\square$

Q.6 Have you ever been educated by a health staff on contraceptive use?<smiles>C1CC2CC12</smiles><smiles>[N+]=[Nb]C1CCC1</smiles>

Q.7 How many times have you had these education (if Yes above)
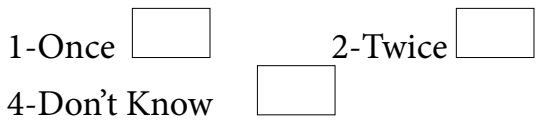

3-several 
Q.8 Have you ever had focused contraceptive education from a health staff?

$1-$ Yes
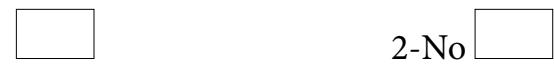

Q.9 In the last one year, have you had any visit by health staff on contraceptive use?<smiles>[Y19]C1CCC1</smiles><smiles>[Y6][N+]1=CCC1</smiles>

Q.10 Do you know where to access family planning information?

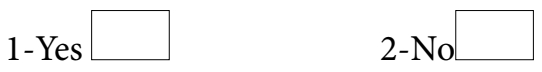

Q.11 Who makes decisions regarding the number of children you would like to have?
1 -Self
2-Husband
3-Both spouses

4-Extended family members

Q.12 Who decides when you should give birth?

1-Self $\square \quad$ 2-Husband $\square$ 3-Both spouses $\square$

4- Extended family members

Q.13 Who makes decisions regarding the use of contraception?

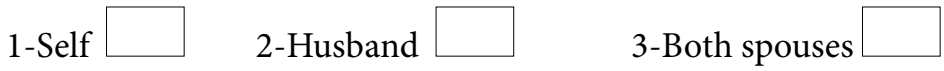

4- Extended family members

Q.14 Does cost have an effect on the choice of family planning method you use? 1- Yes $\square$ 2- No $\square$

Q.15 Have you ever discontinued family planning use due to your inability to pay?<smiles>[Y10]C1CCC1[AsH2]</smiles> 


\section{Section C \& D}

\section{Access on demand and future use intentions for contraception}

This part assesses current and previous user's account of having access on demand for contraceptive use. Questions also elicited participant's future intentions for future contraceptive use.

Q.1 Do you have access to family planning services on demand?
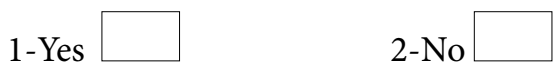

Q.2 Who makes decisions regarding the number of children you would like to have in your lifetime?

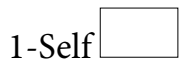

4- Extended family members

Q.3 Who decides when you should give birth?

1-Self 2-Husband

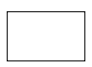

3-Both spouses

4- Extended family members

Q.4 Is your partner in support of your current level of use of family planning?

$1-$ Yes

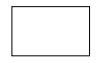

$2-\mathrm{No} \square$

Q.5 Do you get satisfaction from family planning use at the point of provision?

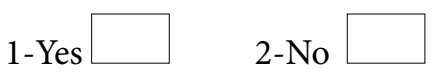

Q.6 Are the hours the facility open convenient for your use?

1-Yes

2-No<smiles>C1CCC1</smiles>

3-Don't know 
Q.7 What times is most convenient for you?
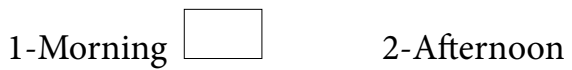

3-Evening $\square$

4-Other $\square$

Q.8 What is the attitude of providers in the provision of contraceptive services?

1-Satisfactory

2-Nonsatisfactory $\square$

3-Poor $\square$

4-Bad

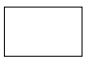

5- Don't know

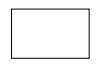

Q.9 Does cost have an effect on the choice of contraceptive method you use?

1 -Yes

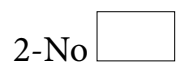

3-Don't know

Q.10 Do you wish to continue the use of contraceptives in the future?

1-Yes

2-No 


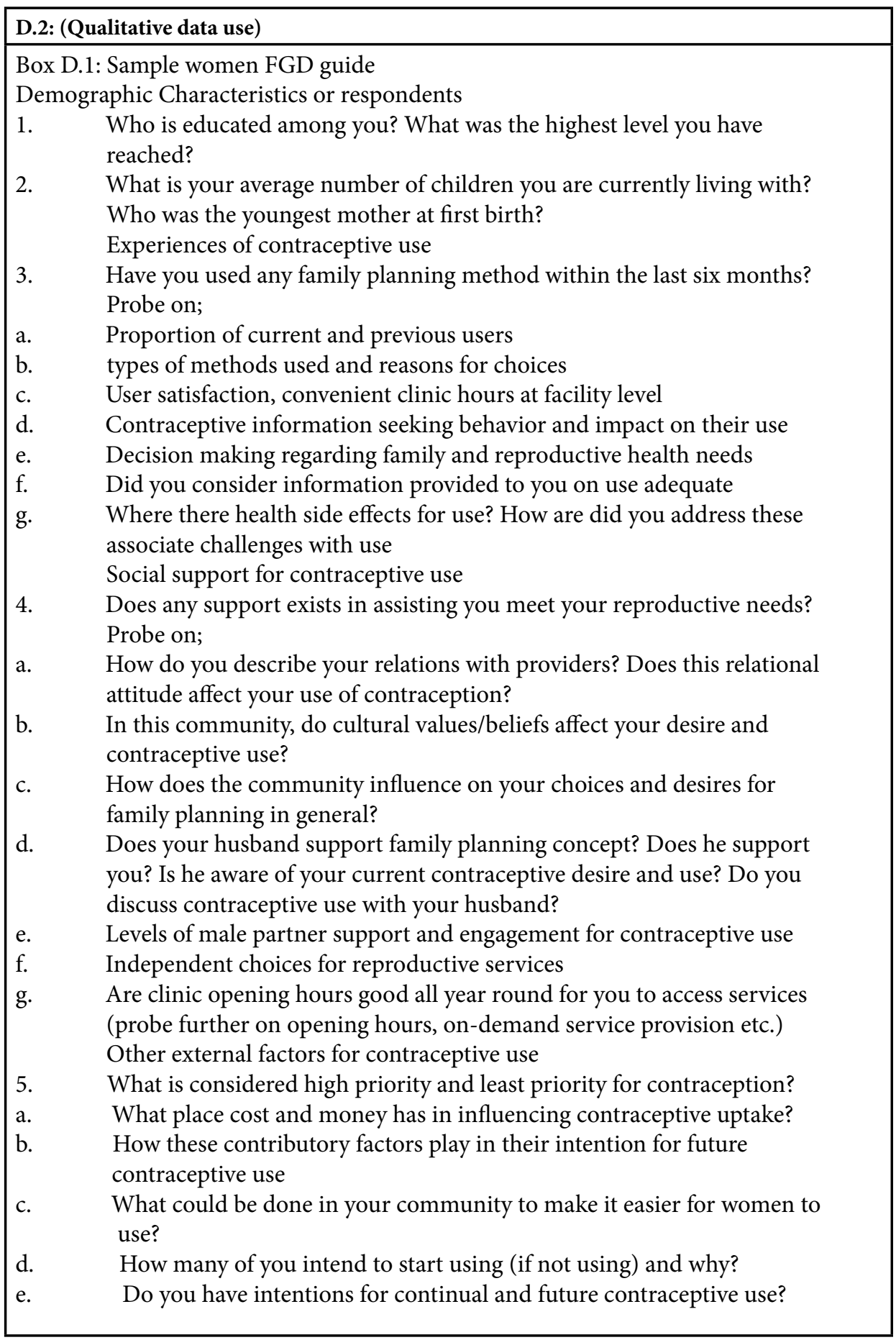


Box D.2: Sample men FGD guide

Demographic Characteristics

1) Probe on the following and record

Marital status, Religion, level of education (Probe further on these socio-demographic effects on their current view of contraception) Knowledge, awareness and practices for contraception

2) How many of you here have heard about family planning (contraception)? Probe on;

a. Knowledge, awareness levels and their current and previous use issues.

b. Probe for methods known, level of use and where you obtain your services

c. Probe to find out why others do not use or encourage others to use

d. I will now read you some statements about contraception. Please tell me if you agree or disagree with each one.

- Contraception is women's business and a man should not have to worry about it.

Women who use contraception may become promiscuous.

Having too many children may be dangerous for a woman's health

- It is better not to have more children than we can afford

- Children in smaller families are more likely to stay healthier and have good start in life

Contraceptive use support

3) Will you/do you support your wife(s) who want to use contraception? If YES Or NO what are reasons behind your action. Probe on;

a. Women perception on husband opposition for use

b. Husbands views on women perception on them

c. Men's perception about covert users

d. Men's social cultural context factors influencing family planning concepts

e. Discuss any issues you have with the use OR Services provided in your community? 
Box D.3: Sample midwives IDI guide

Young women preferred choices for use

a) What factors drive women use of contraception under your clinic setting

b) How does your outfit support women to meet information seeking for contraceptive use

c) What convenient times/hours do women here prefer to come? What account for those reasons?

d) What method choices are most preferred and why? Probe on: method options for social hindrances for contraceptive use

e) What social context factors define women contraceptive use in your facility? How does these impact contraceptive uptakes at the facility level.

f) How will you rate (high or low) male support/ opposition for contraceptive use at your clinic level? What actions are currently taken to ensure male dominance does not derive women use of contraception? How are men involved to support?

g) Are there other general values/norms that define care seeking for general maternity and reproductive health needs among women? Probe further on types and extent of these factors that affect service utilization.

Institutional shortcomings for contraceptive use

h) Women recount of contraceptive shortages sometimes; how true is this and how does the health system ensures that users don't suffer the consequences after?

i) Provider relational issues have sometimes been describing as bad by women users; what do you think about these views by women? What can providers do to ensure women understand the care provided them?

j) How do you describe the kind of counselling provide for current or intended contraceptive users? Probe further on;

- $\quad$ On demand counselling

- Individual/focused counselling availability and options.

- $\quad$ Providers of counselling and knowledge of counselling.

- $\quad$ Cultural sensitive counselling attributes incorporated for meeting women needs 
Appendix D 
Summary 


\section{Unmet reproductive health care needs among rural women in Ghana}

\section{Summary}

The principal aim of this dissertation was to increase our understand ing of unmet maternity and reproductive care service needs over a woman's life course in rural Ghana. To meet safe motherhood goals in Ghana, it is important to understand contextual unmet demand for reproductive care. Evidence on women's unmet reproductive care is scarce in many developing countries. This challenge in most developing countries could be attributed largely to nonexiting or poorly coordinated vital data registration systems. In Ghana, most targets on maternity and reproductive care capture general measures such as supervised delivery coverage, and antenatal and postnatal coverages. Others include frequency of antenatal care (ANC) visits among others. Furthermore, the adequacy of focused maternity care has minimally been investigated across previous studies in Ghana. This dissertation provides an analysis of the adequacy of maternity care received by women during their last birth. Stakeholder views on maternity care shortcomings and context-specific determinants for contraceptive use are also worth investigating in rural northern Ghana. In addition, we analyse stakeholder's experiences, expectations and decision making processes on reproductive care. In this dissertation, we provide evidence on the above gaps by addressing three principle research questions: what is the adequacy of outcome measures and determinants assessed in previous reproductive studies across West Africa, what are stakeholder views on maternity care shortcomings in rural Ghana, and what context factors drive unmet reproductive care in a rural district of northern Ghana.

This dissertation aims to contribute to a further understanding of unmet reproductive care among rural Ghanaian women. The dissertation consists of seven chapters, summarized here as follows.

Chapter 1 provides a brief history of health sector reforms and key health sector performances on maternity and reproductive care in Ghana. The chapter further outlines the concept of unmet needs and key operational definitions. The history, relevance and key challenge of unmet needs are later examined. In Chapter 1 , we also outline the trends in unmet needs globally and in the Ghanaian context. In addition, we outline the aim, objectives, and methods applied in all chapters of this dissertation. The principle goal of this dissertation is to understand unmet maternity and reproductive care service needs over a woman's life course in rural Ghana. To attain this aim, a review of the adequacy of outcome measures and determinants applied to assess unmet reproductive health needs among women 
in West Africa was undertaken. The chapter concludes with a brief outline of the dissertation.

In Chapter 2, we provide a systematic review of the literature on the adequacy of outcome measures and determinants to assess unmet reproductive health needs among women in West Africa. The review was undertaken for studies published from January 2009-March 2014. Seven databases were searched in this review; PubMed, Econpapers, CINAHL, Psych INFO, Science Direct, Embase and Biomed. In the PubMed search, $\mathrm{MsSH}$ term categories were applied across all key words to ensure relevant publications were extracted for the review. Only English language publications were reviewed. Three broad indicator measures, a) contraceptive use, b) abortion care and c) ANC services use were included in the review. Clinical studies particularly on obstetric care services and reproductive services in relation to HIV/AIDS were excluded from the review. The reference lists of publications were also reviewed and those found to meet the review criteria were added to the list of reviewed publications. In total, 78 publications across seven countries in West Africa were identified and included in our review. The findings from this review show the existence of a broad range but similar outcome measures and determinants for evaluating reproductive care needs in these examined countries. We however found that, a high rate of unmet reproductive care needs still exist across many of these examined countries. This is associated with poor socio-economic indices, deep and varied social norms and beliefs across cultures and traditions that place marriage and a woman's reproductive health needs as the male spouse responsibility at the family, and community level. Our results show poor institutional arrangements divest women reproductive health seeking behavior's across many West African community settings as evidenced in the review. The multiple social, economic, and environmental factors that affect the continuum of reproductive care require multiple stakeholder engagements to bring about health system improvements. Evidence from the review indicates that currently applied measures of women's reproductive health needs might be inadequate in attaining the best maternal outcomes since they appear rather broad. We advocate the need to incorporate country level context measures in benchmarking progress on reproductive health needs among women. This will support a better evaluation in each country level of the progress on meeting women reproductive health outcomes. At the policy level, reforms that improve access (e.g. legislative reforms on restrictive abortion laws) will go a long way to support reduce unmet need levels and improve women reproductive health care needs in West Africa.

Chapter 3 examined maternal care utilization components in Ghana based on the 2008 Ghana Demographic Health Survey (GDHS) dataset. Demographic Health Survey (DHS) data have been collected and analysed in Ghana since 1988. 
The 2008 GDHS was implemented by the Ghana Statistical Service and Ghana Health Service with technical support from ICF Macro and MEASURE DHS program. In total, data from 2,147 eligible women who reported childbirths 5 years prior to the study were analyzed. Using a cluster analysis methodology and WHO guidelines on focused maternity care, we provide a segmentation of woman's met and unmet care needs at last birth. Three classifications assessed maternity care adequacy: adequate, less and east adequate care. Overall, more than half of Ghanaian women received adequate prenatal and postnatal care. Worrying was the equally approximate share of women who received less and least adequate care. We found that focused care components in the overall antenatal utilization were low compared with less or least adequate care groups. We also establish that the frequency of antenatal visits is not associated with the adequacy of care received. Our cluster analysis findings reinforced that women, who were attended by skilled personnel, were more likely to report adequate care irrespective of the frequency of antenatal visits reported. Furthermore, the indirect impact of the lack of public health insurance was positively associated with least adequate care reported by women. Across regions and within rural settings in Ghana, disparities exist, which is often compounded by supply-related factors. Efforts at addressing skilled workforce shortages, greater accountability for quality and equity, and those that improve women urgency, choices and active participation in reproductive care decision making are important policy strategies to improve maternity care outcomes in Ghana. We advocate that the broadening of opportunities for women beyond having a say on their health needs can help alienate socio-cultural values that devalue women reproductive care seeking. In addition, we posit the need for long term planning and systemic reforms on health insurance membership for maternity care policies in Ghana. Improving health system arrangements on skilled professionals and curricula trainings for health staff would further enhance the quality on focused maternity care in Ghana. Given the potential benefits of health insurance ownership and use for improved prenatal and postnatal care outcomes, there is an urgent need to improve structural deficits under the current National Health Insurance Scheme in Ghana. Improving structural deficits under the health insurance scheme would improve user confidence among non-active members who have lost out due to issues of reported inefficiency and poor quality services delivery nationwide.

Three stakeholder group views on shortcomings for maternity care are examined in Chapter 4. This aim evolved from the understanding that formal networking and stakeholder interactions are essential to meet patient-centered maternity care needs. We first explored women's views and juxtaposed these with the views of frontline health care staff (midwives/nurses/community health officers) and public and private policy decision actors at regional and district 
levels. An ethnographic design was adopted which enabled a simultaneous reconstructing of lived stakeholders experiences. This approach also enriched the process of understanding stakeholders' arguments on shortcomings for maternity care in rural Ghana. Two kinds of qualitative field data were collected in the same districts during this period of the dissertation. This was related to: a) shortcomings in maternity care provision and use, and b) reproductive care experiences, expectation and decision making processes. The first component was analyzed in Chapter 4 for this dissertation while the latter was analyzed in Chapter 6 of the dissertation.

Our findings presented in Chapter 4 reflect a broad range of factors on maternity care shortcomings. Emergent themes include factors related to social arrangements in the community, health system (payments and provision of maternity care) and health policy frameworks for service provision. Despite differences in stakeholder opinions, many shortcomings put forward in this study, reflect consensus on maternity care shortcomings. Socio-culturally, women's reproductive seeking behavior is influenced by male and family relational environments that devalue women choices on reproductive care. Travel time, direct and in-direct costs and poor facility level conditions are major barriers to maternity care seeking. Poor facility level conditions, poor focused care, and inhumane treatments impugned by health staff were also reported by women. Also, reports of poor bedside manners, poor delivery room environments (including noisy delivery room environments) were cited. In addition, stakeholders admitted that knowledge and skills for both mother and new-born care is a determinant for a woman's trust and use of facility birthing services. Within the policy framework for improving maternity care, the culture of "gift assurance" as coined by women is espoused. However, the extent to which this impacts on the equity dimensions of maternity care was not examined in this dissertation. We advocate inclusiveness and the effective dissemination of patient care delivery standards among women and health staff at all levels. This has the potential to create a culture of trust, transparency and openness regarding ways to tackle barriers for adequate care provision and seeking. Aside this, our results show that maternity policy strategies such as the free-fee delivery policy in Ghana, have not been able to tackle indirect costs for maternity care. At the individual level, patient-staff conditions that create a culture of trust and respect should be advocated to improve patient-centered care and address traditional roles and social norms in birthing practices. Above all, a strong political environment that improves the rural road infrastructure and takes steps to ensure a more effective decentralized national ambulatory service system will better ensure women effectively benefit from the country's free maternity care policy.

Chapter 5 outlines the context-specific factors associated with contraceptive use in a rural district of Ghana. The study employed a descriptive cross-sectional 
design. Data were collected between February and March 2012. Three main measures were investigated; current contraceptive use, access on demand for contraceptives and future use intentions for contraception. An individual woman access to, and use of contraception is influenced by clinic arrangements. Furthermore, young women's satisfaction with contraceptive use significantly impacts current user intentions. The results show a user preference for friendlier clinic working hours. Contextual factors such as focused counselling/education further enhances women contraceptive use abilities. We argue that adequate and timely education assists women to attain the principle of the "calculus of conscious choice" with contraceptive use. In addition, focused counselling provides an alternative route for many rural context health providers to reach underserved and un-educated population groups with contraceptive products. We advocate that integrated health services delivered in rural settings should include a critical component of individualized counselling for women. Despite the dominant patriarchy roles in rural settings that impact reproductive care seeking, focused counselling plays an important role in upscaling user satisfaction and use for contraception. Our results support the need to provide client centered, culturally sensitive and acceptable care that respects women's preferred convenient clinic hours for contraceptive use. At the wider policy level, clinic settings must design structures that guarantees continued confidentiality and provide mechanisms to address individual health concerns after use. At local community level, health advocacy and campaigns must address poor health seeking behavior that perpetuate myths and misinforms clients about safe and beneficial contraceptive clinic services.

In Chapter 6, we mapped out varied stakeholder views on reproductive care services use in rural Ghana. Specifically, stakeholder experiences, expectations, and policy decision making processes for reproductive care in three rural districts were examined. Ethnography was adopted as a study design. The methodology enabled a simultaneous reconstruction of stakeholders' experiences among three stakeholder groups: women, health staff and policymakers. Four themes emerged: the impact of gender roles on reproductive care needs, experiences with meeting reproductive care needs, expectations about reproductive care needs, and the policy setting and decision making processes on reproductive care needs. On the gender role impact for contraceptive use, our results point to the fact that male perceptions on sexual passivity of women drive male beliefs and desire to control women sexual behaviors. Complementarily, where male spousal control neglects women's needs, covert use of reproductive care services exist. Women reported health staff cluttered control mechanism limits use of reproductive care. Chapter 6 demonstrates that health staff exercises control and supremacy and this has the tendency to shy women away from meeting their reproductive care needs. Reproductive care experiences show that reproductive services non- 
differentiation at the point of service provision influences individual reproductive choices and use. In addition, fee payments during post abortion care and management is reported as a major barrier to health facility utilization. We also found that social stigma can reduce user's willingness to report poor reproductive experiences at the health facility level. Where reproductive services for STIs, abortion/post-abortion care, and infertility treatments services are limited in scope, myths on what defines sexuality and womanhood in the society further foil social stigma on care seeking among women. Additionally, the non-availability of psychological support at health facility centres lead to low self-esteem among reproductive care users. Stakeholder's expectations differed on what levels of counselling were adequate to meet user reproductive care expectations. Women's views on counselling services differed from that of health staff and policymakers. Health staff and policymakers stated that policy processes for decision making on reproductive care services is driven by previous and current donor funding needs. The two stakeholder groups also acknowledged the difficulties in prioritizing local reproductive care needs at health facility levels. Additionally, all stakeholder groups stated the need to design health systems that are more integrative and supportive on reproductive care delivery. Systems and structures that create conditions of mutual respect among provider-user, and empower women in their healthcare seeking behaviors also need to be encouraged. Stakeholder advocate for clear guidelines on reproductive care to be contextualized to meet user care needs. We stressed the need for efforts to delimit cluttered relationships that exist between health staff and reproductive users at facility levels. We also propose the inclusion of post-abortion care and post-pregnancy termination management issues into the National Health Insurance benefit package. A holistic health system that ensures sexual and reproductive health needs are truly subsidized or provided essentially at lower fee cost will benefit rural Ghanaian population groups.

Chapter 7 discusses the main results of Chapter 1-6. We also reflect on the policy implications our results have on reproductive care in Ghana and beyond. From this dissertation, reproductive health targets related to service quality and user satisfaction are drivers of reproductive health service utilisation among women in West Africa. This dissertation also shows that reproductive care quality and satisfaction have the tendency to determine reproductive care use and caring practices received from health care providers. Quality and user satisfaction attributes enumerated here support existing evidence that patient satisfaction is indispensable to quality improvements in the health systems. Our review indicates that the quality of reproductive care outcome estimates remains largely documented at the clinical and patient reported levels. In addressing service quality and user satisfaction needs for women, tailored policies to improve 
poor health system arrangements for reproductive care service delivery remain persuasive to meet user's satisfied demands for reproductive care.

Furthermore, focused reproductive counselling must be integrated into interventions aimed at scaling up family planning and broad reproductive health services use in rural Ghana. Even among women without formal education, focused reproductive counselling addresses contraceptive misconceptions, myths and wrong cultural norms on fertility control. Focused reproductive counselling also has the propensity to enhance women's contraceptive autonomy by expanding their productive reproductive health choices. In addition, prompt and adequate focused reproductive counselling facilitates health staff and user's shared decision making on reproductive care service use. Clinic counselling services must move beyond the health system and recognise the social patriarchal dimensions for reproductive services delivery. In addressing women's contraceptive needs in a given context, clinic settings must design structures that guarantee not only confidentiality, but suitable working hours to further enhance women access and use for services all year round. Also, the provision of reproductive care services must be more client-centered and respond to culturally sensitivities of the population in need.

The significant role of health insurance in influencing cost and care adequacy levels among women in Ghana is also highlighted in this dissertation. Specifically, insured women were more likely to seek several components of maternity care compared to non-insured in Ghana. We also show that regional variation in health insurance access and use influence disproportionally the adequacy of maternity care across all regions in Ghana. In environments where women report travel time and financial cost barriers as impediments for maternity care utilization, greater disparity for general health use is hardest among women living in rural dwellings. Although the insured will be more likely to use health services at times of need, insured individuals may at times use more services than required (moral hazard).

The frequency of antenatal visits alone does not correspond with focused maternity care quality and user satisfaction. Thus, focused maternity care during antenatal periods will depend very much on the care components delivered and the times spent in addressing individual health needs and concerns during antenatal. Our results emphasize the need for a careful application of the WHO recommended minimum of four visits among women identified as low risk pregnancy during the first trimester visits for ANC.

The factors impacting reproductive and maternity care in rural Ghana are multifaceted and complex, operating at household, community, and health system and policy formulation or coordination levels. Hence, the need to strengthen 
not only the institutional care infrastructure, but improve social conditions and facilitators to deliver effective reproductive care services at point of service use. Evidence espoused for this dissertation show health system barriers that need policy reformation and coordination to improve reproductive inequalities in rural Ghana. We state that policy processes for informed decision making on reproductive care services is largely driven by previous and current donor funding needs. Stakeholders acknowledged difficulties in prioritizing to meet local reproductive care needs at facility levels is partly due to donor funding schemes. Stakeholders' strongly advocated for clear guidelines on reproductive care to be contextualized to meet user care needs. Health counselling and the need for individualized care have increasingly been evidenced as an entry approach to reach new users of health services.

More so, providing patient-centered, culturally sensitive and acceptable care that respects women's preferred convenient clinic hours enhances reproductive service use. These also have the propensity to improve reproductive user satisfaction. Agreeably, delivering patient-centered reproductive health care services require multiple stakeholder involvement and integrative and supportive health systems. We illustrated that patient-centered care is difficult to attain, where supply related factors such as adequate health staff and psychological support is lacking. This dissertation did not establish a direct relationship between patientcentered care and overall health benefits. However, there is ample evidence to show that, patient-centered approaches play an important role in attaining positive reproductive health outcomes for women. Reproductive counselling services must be designed to be user-specific.

In providing support for enhanced decision making, health system designs that allow continual feedback and psychosocial support among users and improved professional skills to deliver the needed care are very important. Our results advocates for inclusiveness and the effective dissemination of patient care delivery standards among stakeholder groups involved in the provision and use of services. Such culture of inclusiveness has the potential to promote trust and mutual respect required in the provision of patient-centered reproductive care services. 



\section{Thank you words}

As I bring to a memorable end my $\mathrm{PhD}$ work in Maastricht, words cannot express how gratifying I am in a position to thank the many people that touched my life and made this journey worthwhile. To GOD be glory and, honor given. Amen

To Prof. dr. Wim Groot and dr. Milena Pavlova, this journey of a PhD in Maastricht would have not been attained without you believing in what I wanted when I first made contact with you both over my PhD study. Milena, I believe you were God sent when you offered a reply on my request for a supervisory team for my $\mathrm{PhD}$ Scholarship that was almost terminated due to my inability to enrol for my $\mathrm{PhD}$. Although Netherlands was not a first option in my initial applications, I had to abandon all the rest later when I realized how positive and enthusiastic you attached to my request for study. Your speedy response and subsequent discussions with Wim that finally pulled me to Maastricht is astonishing, and I will forever remain grateful. Wim, I remember the first skype call we had when eventually you approved my request for you and Milena to supervise me. It was brief, humbling and carried a fine level of cordiality that told my spirit that I was with the right team for my $\mathrm{PhD}$ work. This finally saw me arriving in Maastricht on the 9th February, 2014 to begin this Journey. To both of you, I want to say thank you. I came here straight as a development worker in the field who was expected to write lengthy field reports and minutes. Am leaving Maastricht today confident of a place in academics. Wim, in moments things seemed unclear, just a word in our weekly meetings made everything looked simple. You are an academic with few words that changes the beauty around us. Thank you. Milena, I took my strength from you in this $\mathrm{PhD}$ trajectory. Your strength, multitask abilities is admirable. Thank you. I remember many moments in the $\mathrm{PhD}$ trajectory, Wim and Melina you would often say I was progressing well in my $\mathrm{PhD}$ trajectory. Well to be honest, I wanted to see the end of the road in this journey to believe. I am convinced now of that progress and the fantastic supervision that supported this work through. Although I tried hard to believe of my own progress, I only hoped and waited for a day like this to fully realize the positive feelings of 'progressing well' in my $\mathrm{PhD}$ diploma. Perhaps, all I believed was to be writing these concluding remarks to be fully convinced I was on course. Thank you Wim, Thank you Melina, for seeing and believing in me ahead of time. To dr. Regien Biesma, thank you for the lovely days in Dublin and for your critical review on the papers we authored together in my $\mathrm{PhD}$ trajectory.

Am ever grateful to my parents, Mr. and Mrs. George Ayanore for the many sacrifices and journeys we have had together in this life. Dad and Mum, your sacrifices and support brought many impossible ways possible in my life. To my lovely sister Teni, and brothers, Nsoh, Peter, Francis, Jonas, Augustine, Sebastian and Adua, God richly bless all of you in measure for your support to me in this 
life. To Professor Thomas Mba Akabzaa, who advised me to take a first shot at University education, and who made this study in Maastricht very possible, I say thank you.

To lovely wife, Ellen, my precious and faithful source of strength, the very model of a woman described in Proverbs 31:10-29, Thank you for been there for me. Thank you for trusting me when no one else did. For the many hard times you kept the family together when I was away in Maastricht, you are such a germ. I dedicated this $\mathrm{PhD}$ work to my children. To my son, Akanboe and daughter, Asomkateere. Even though you were young to easily notice my absence from home anytime I travelled to Maastricht, your portraits on my working space kept reminding me that I was far away from home. Thank you for this reminder that propelled me on during my $\mathrm{PhD}$ work in Maastricht.

To my family in Maastricht that created a lovely atmosphere during any of my period of stay in Maastricht, am grateful. To our father in the Lord, Pastor Benjamin Odetola, and our mother in the Lord, Janet Odetola, words cannot express my thankfulness to God for planting a home in Bethel for me. Where ever I am, and who ever I become, Bethel story will be told. To my two paranymphs, Emmanuel and Victor, am grateful for your support in this journey of mine in Maastricht. Martin, Davina, Elvis, Jesutofunmi, Jesutomiwo and Testimony, bro Felix and family, bro Dami and family, all bethel families in Belgium, and MsM alumni of the bethel family. My encounter with the bethel family was life transforming. My prayer is every soul finds peace and a place in Heaven.

I will also like to thank my office colleagues, Ben and Keilong for moments of laughter, joy and space we shared together. I cannot forget our usual spareribs trips, movie night and dinners we shared together. Thank you both for the memorable times we shared together. To dr. Chaw-Yin Myint, thank you for always willing to read and provide direction when I needed an extra eye on my work. To my senior colleague dr. Reza Rezayatmand who was the first to meet up and show me to the department after my arrival, my special thanks to you and your family in Iran. Reza, you provided warmth for me to settle well in Maastricht. Thank you.

To Suus Koene, Brigitte Caenen, Janet Verheijen, Joanna Houtermans, and WillyAnne Kanters, I never got lost in anything within the department. Thank you all all for the support offered me at every step of my working days in the Department

I cannot conclude without thanking the members of the evaluation committee that evaluated the content of this dissertation, am grateful for guiding and providing critical comments for improving the final stage of this dissertation. To Roland Owusu-Tabi for the layout and cover design, I am most grateful for the hours spent on this piece to get it to its final stage 


\section{Curriculum Vitae}

Martin Amogre Ayanore was born on 2nd November, 1983 in Bolgatanga, Ghana. He graduated from bachelor degree in 2008 after which he served as teaching Assistant with Bolgatanga Polytechnic between 2008-2009. He obtained a postgraduate diploma in Education teaching qualification with the University of Cape Coast, Ghana in 2009. Between 2011 and 2014, he worked as WASH and development facilitator with World Vision International, Ghana. Prior to that, he served as program manager and senior programme manager for WEMNETGhana.

Since January 2014, he worked as an external $\mathrm{PhD}$ candidate at Maastricht University. Martin is passionate about evidence based research that focuses on child and maternal health, neglected sexual and reproductive rights among vulnerable population groups. This drive led him to work on the project unmet reproductive health care needs among rural Ghanaian women. In 2016, Martin worked at the Royal College of Surgeons Ireland as visiting $\mathrm{PhD}$ candidate to the Department of Epidemiology and Public Health Medicine for two months under the direct supervision of dr. Regien Biesma.

Martin co-founded the think-tank, Centre for Health Policy, Innovation and Research (CHPAIR-Africa), a think-tank based in Ghana committed to providing evidence based research for policy and advocacy for better health outcomes across the African continent. He is currently the Chief Executive Officer of CHPAIRAfrica.

At present, he teaches at the Department of Family and Community Health, School of Public Health, University of Health and Allied Health Sciences, Hohoe, Ghana. His teaching roles include courses in health financing, global health security, and public health program monitoring and evaluation among others. Martin is married with two children. 



\section{Publications}

Ayanore, MA., Pavlova, M., Biesma, R., \& Groot, W. (2017). Stakeholders' views on maternity care shortcomings in rural Ghana: An ethnographic study among women, providers, public and quasi-private policy sector actors. The International Journal of Health Planning and Management, DOI: 10.1002/hpm.2411.

Ayanore, MA., Pavlova, M., \& Groot, W. (2016). Focused maternity care in Ghana: results of a cluster analysis. BMC Health Services Research, 16:395. DOI: 10.1186/ s12913-016-1654-5

Ayanore, MA., Pavlova, M., \& Groot, W. (2016). Unmet Reproductive Health Needs among women in some West African countries: A Systematic Review of Outcome Measures and Determinants. Reproductive Health, 13:5 DOI 10.1186/ s12978-0150104-x

Ayanore, MA., Pavlova, M., \& Wim Groot, W. (2016). Context-specific factors and contraceptive use: experiences of young women in a rural district of Ghana. Under Review

Ayanore, MA., Pavlova, M., Biesma, R., \& Groot, W. (2017). Stakeholder's experiences, expectations and decision making on reproductive care: An ethnographic study of three districts in northern Ghana. Under Review

\section{Other publication works}

Ayanore, MA., Kuganab-lem, R., \& Paul Armah Aryee (2016). Beyond our labour pains: women experiences of informal payments and delivery room conditions at birth in the Bongo district of Ghana. International Journal of Community Medicine \& Public Health. 2016; 3(7): 1695-1706 DOI: 10.18203/2394-6040. ijcmph20162031

Adoliba, AA., Ayanore, MA., \& Aaron Kampim (2016). Organizing to meet critical needs for maternity care in developing countries: a social and health system diagnosis. Medico Research Chronicles; 3(2), 232-239

Ayanore MA., \& Paul Armah Aryee (2015). Use and determinants offamily planning among young Women (18-28 Years) attending health facilities in GaruTempane district of Ghana. International Journal of Health Sciences, Vol. 3, No. 4, pp. 1-6. DOI: $10.15640 /$ ijhs.v3n4a1.

Nyefene, MK., Ayanore, MA., Adatara, P., Groot, W., \& Pavlova, M. (2016). Barriers to the use of facility-based birthing services in rural northern Ghana: the perspective of key stakeholders. Under review 
Ayanore, MA., Avenyo, EK., Kwadwo, VO., Kwaku, M., and Victor Mogre. Social health insurance and birthing care outcomes in Africa: evidence from recent demographic health surveys in Ghana, Namibia, and DRC. Under Review

Ayanore, MA., Amuna, NN., Awolu, A., Aviisah, MA., Akpalu, B., Mogre, V., Adoliba, AA., and Richard Ofori-Asenso. Towards resilient health systems in Africa: a systematic review of health workforce, surveillance and health governance issues for health systems strengthening. Under Review

Ayanore, MA., Kampim, A., Aviisah, MA., Amuna, NN., and Ankobil Amandus. Labour referral for birthing care: a sequential mixed method approach of community and health system arrangements in Ghana. Under Review

\section{Oral conference presentations}

2015 Public Health Conference. Oral online presentation on maternity care in developing countries.11th-13th July, 2015.Bangkok, Thailand 14th Annual International Conference on Health Economics, Management \& Policy. Athens Institute of Education and Research Athens, Greece.

Oral paper presentation on "Unmet Reproductive Health Needs among Women in West Africa: Systematic Review of Outcome Measures and Determinants". Greece, June 20-24 June 2015.

Poster presentations 7th African Population Conference, Poster presentation on Unmet Reproductive Health Needs among Women in West Africa: Systematic Review of Outcome Measures and Determinants. Johannesburg, South Africa, 30 November-4 December 2015.

International Institute of Health Economics and Management. 2015 International Conference on Health, Economics and Management: Poster paper presentation titled: Focused maternity care in Ghana: Results of a cluster analysis. Augsburg, Germany, 16th-18th September, 2015.

Symposia invitations/presentations Panelist on one day symposium on Sexual Reproductive Health and Rights and Sustainable Development Goals in SubSaharan Africa. International Development Studies Alumni Association, The International Development Studies (IDS) group in the Department of Human Geography and Spatial Planning, Faculty of Geosciences at Utrecht University. Utrecht, Netherlands, 4th September, 2015.

Health in Africa and the Post-2015 Millennium Development Agenda: 3 days Symposium University of Illinois at Urbana-Champaign. Paper presentation titled: Focused maternity care in Ghana: Results of a cluster analysis. University of Illinois at Urbana-Champaign, USA, 20th -22th May, 2015. 


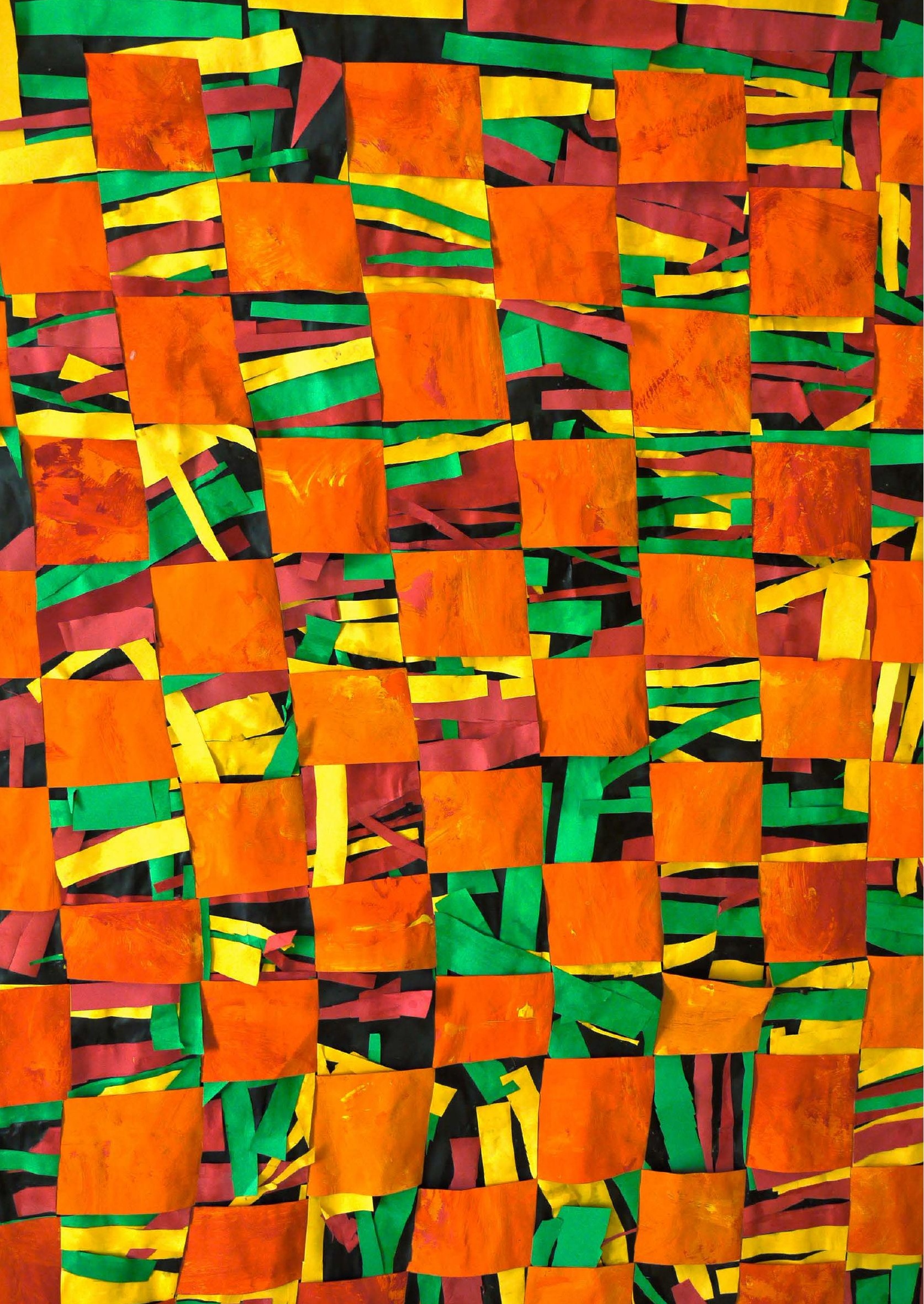

